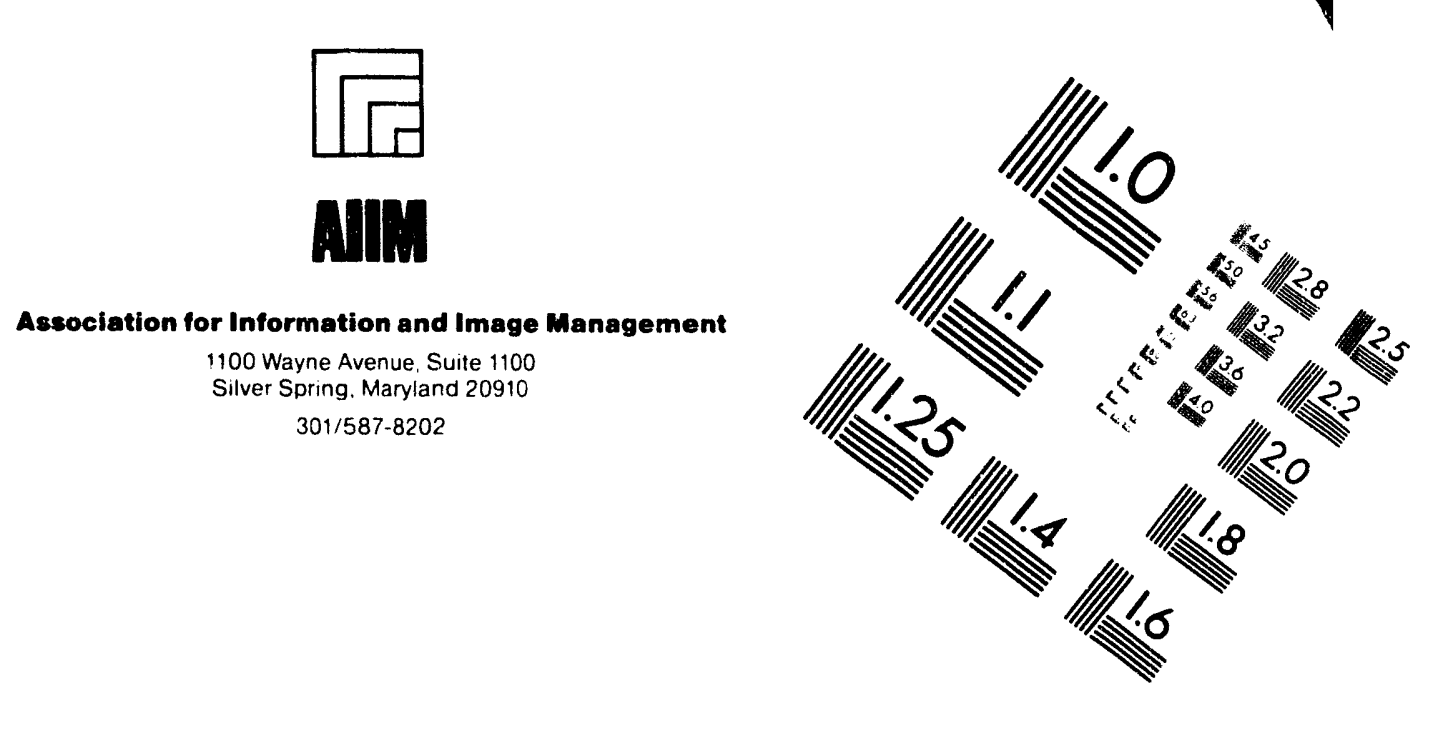

\title{
Centimeter
}

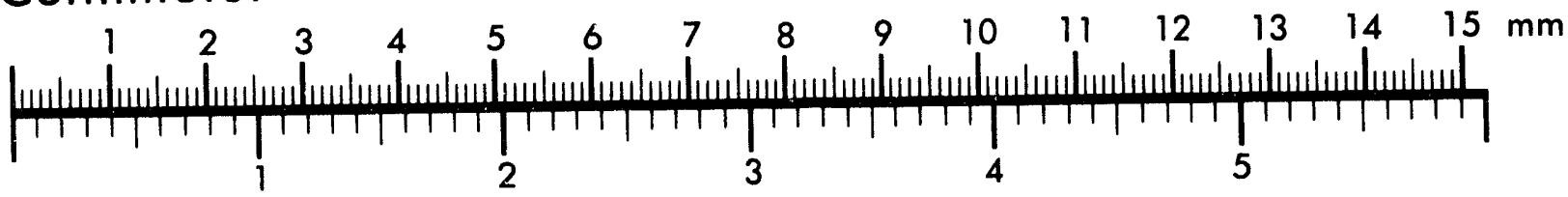
Inches
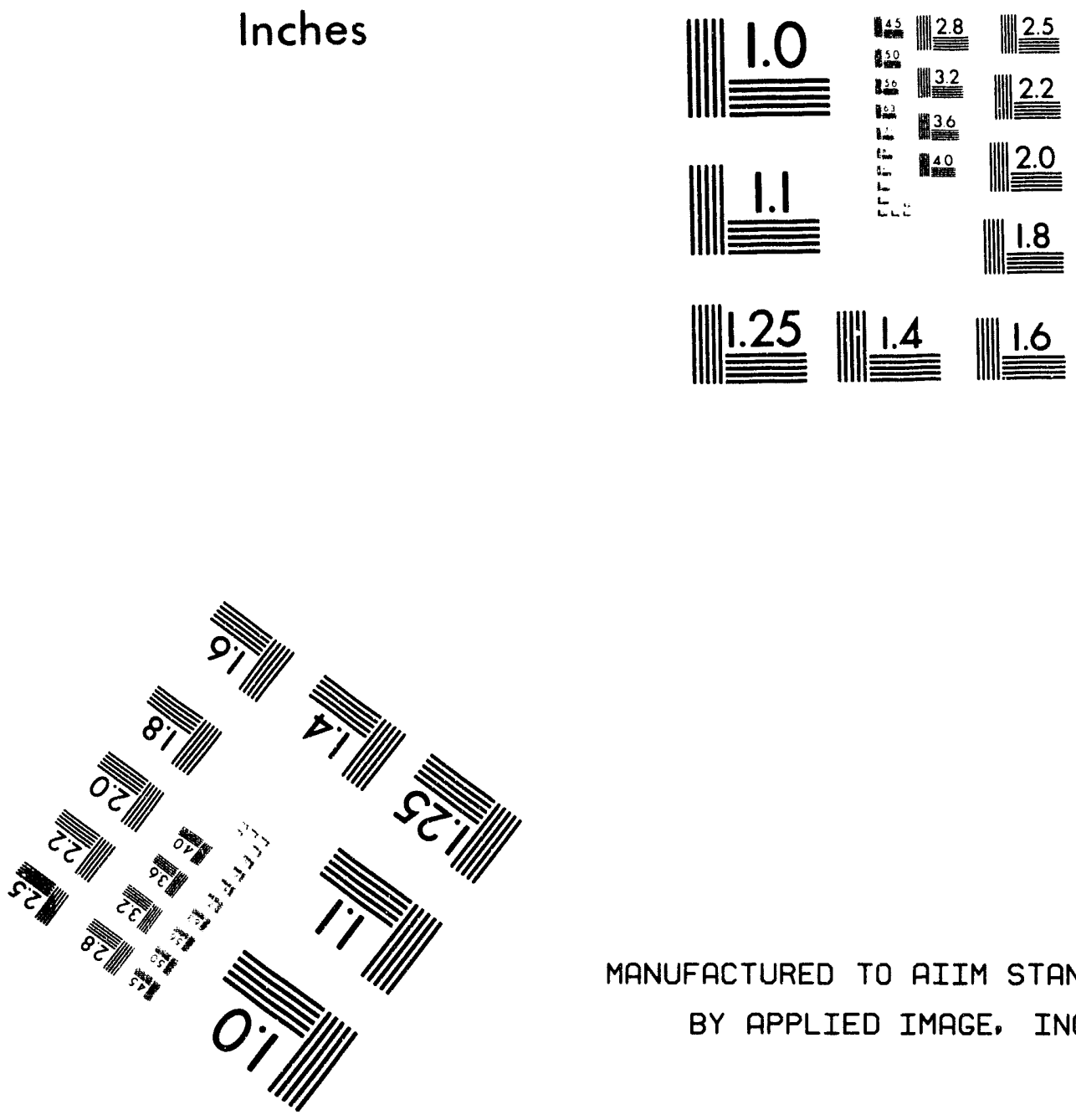

MANUFACTURED TO AIIM STANDARDS

BY APPLIED IMAGE, INC.

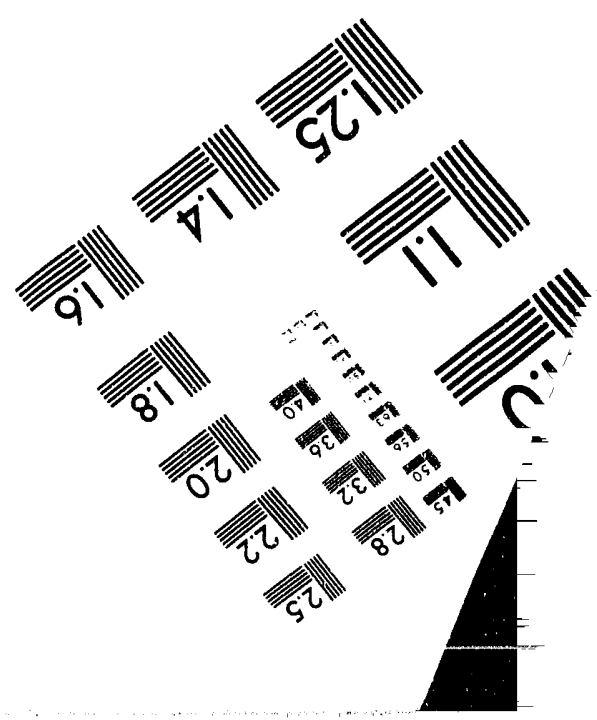



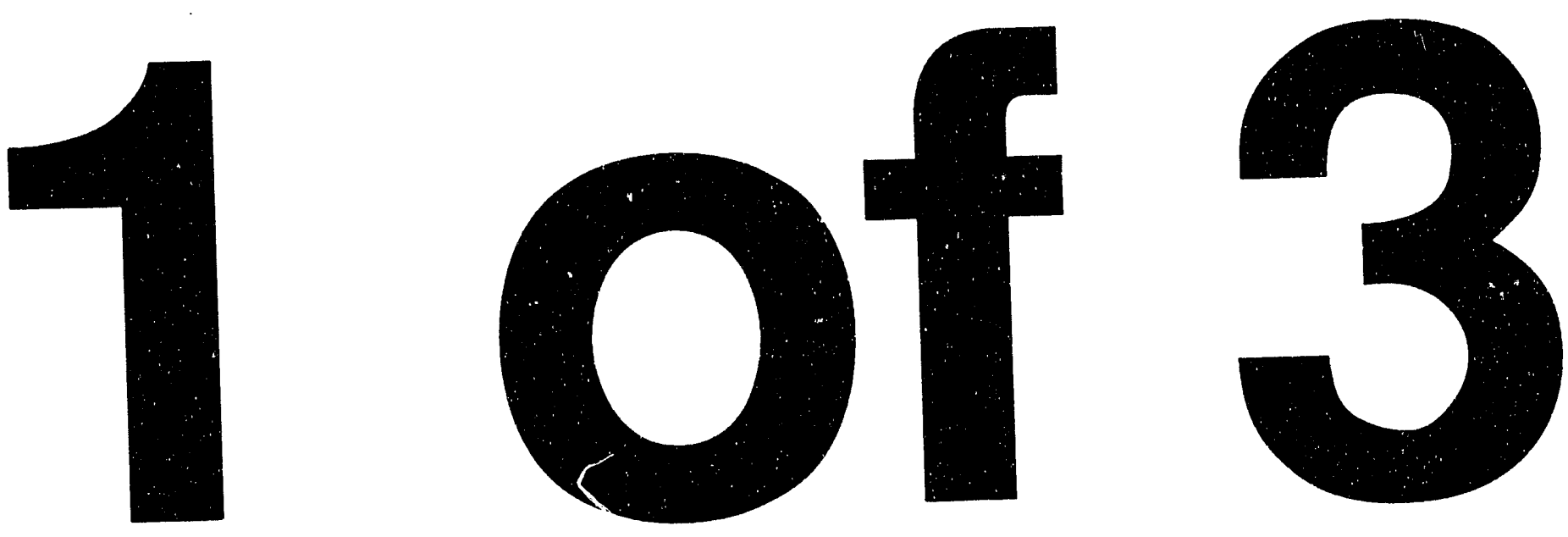


\section{PROCESSED SEISMIC MOTION RECORDS FROM DESERT HOT SPRINGS, CALIFORNIA \\ EARTHQUAKE OF APRIL 22, 1992 \\ RECORDED AT SEISMIC STATIONS \\ IN SOUTHERN NEVADA}

April 1993

Peter K. Lum

Kenneth K. Honda

Prepared for

U.S. Department of Energy

Nevada Operations Office

Under Contract DE-AC08-89NV10733

Prepared by

URS/John A. Blume \& Associates, Engineers

100 California Street, San Francisco, CA 94111

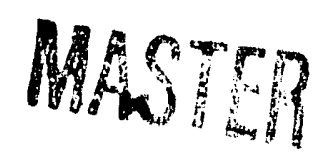




\section{DESERT HOT SPRINGS, CALIFORNIA \\ EARTHQUAKE OF APRIL 22, 1992}

\section{Background}

As part of the contract with the U.S. Department of Energy, Nevada Operations Office (DOE/NV), URS/John A. Blume \& Associates, Engineers (URS/Blume) maintained a network of seismographs in southern Nevada to monitor the ground motion generated by the underground nuclear explosions (UNEs) at the Nevada Test Site (NTS). The seismographs were located in the communities surrounding the NTS and the Las Vegas valley. When these seismographs were not used for monitoring the UNE generated motions, a limited number of them were maintained for monitoring motion generated by other than UNEs (e.g., motion generated by earthquakes, wind, blast). During the subject earthquake of April 22, 1992, the systems located at 19 ground locations recorded the earthquake motions. Due to the potential benefit of these data for the scientific community, DOE/NV authorized the release of these records.

\section{Earthquake Parameters}

The earthquake parameters obtained from various sources were:

$\begin{array}{ll}\text { Epicenter: } & 33.96^{\circ} \mathrm{N}, 116.32^{\circ} \mathrm{W} \\ \text { Depth: } & 12 \mathrm{Km} \\ \text { Magnitude: } & 6.1 \mathrm{M}_{\mathrm{L}} \\ \text { Origin Date/time: } & 23 \mathrm{April} 1992 \text { Day } 114 \mathrm{Hr} 04 \text { Min } 50 \text { Sec } 22 \text { (GMT) } \\ \text { Local Date/time: } & 22 \text { April 1992 9:50 p.m. (PDT) }\end{array}$

The earthquake was reported to have occurred under the Little San Bernardino Mountains about $17 \mathrm{~km}$ east of Desert Hot Springs and $22 \mathrm{~km}$ northeast of Palm Springs. The earthquake magnitude was estimated to be $6.1 \mathrm{M}_{\mathrm{L}}$. The earthquake was felt throughout the communities in Las Vegas and other southern Nevada areas.

\section{Description of Equipment}

Three types of seismographs were used in the monitoring program. They were L-7 velocity meter manufactured by the Precision Instrument Company (L-7B system) and by Develco, Inc. (L-7D system); PDR-1 by Kinemetrics; and PDAS-100 by Teledyne Geotech. The FBA-11 accelerometers manufactured by Kinemetrics were utilized as sensors for both the PDR-1 and PDAS-100 systems. The FBA-11 has a flat response from DC to $50 \mathrm{~Hz}$.

L-7 Velocity Meter: It is a continuously recording velocity seismograph which records the motion on an analog magnetic tape. The system has a flat velocity response over a frequency spectrum from $0.1 \mathrm{~Hz}$ to $34 \mathrm{~Hz}$ (Reference 1). It has a recording capability of 5 continuous days.

PDR-1: The acceleration signal from the sensors is converted to a digital form and recorded on a digital cassette tape. The selectable sampling rate was set at 200 samples per second per 
offs would partially filter-out this long period motion. In order to examine the characteristics of the long-period motion, the lower cut-off frequency was extended to $0.05 \mathrm{~Hz}$ and the PSRV was extended to a period of 30.11 seconds. Of course, the PSRV values for periods greater than the cut-off frequency or the beyond the recording system response are not the optimum values.

- In the final phase, the records were arranged for the long-term preservation and for report preparation. The final product contains data plots in chronological order of data processing; original recording, uncorrected and corrected Fourier amplitude spectra, corrected acceleration, velocity, displacement and PSRVs.

\section{Results}

Table 2 tabulates the uncorrected peak acceleration or velocity, corrected peak acceleration, velocity and displacement values and any data processing comments. Some of the problems encountered were late trigger (the system was not triggered by the initial arrival, but by larger amplitude motion which arrived later), noisy record (some of which could not be removed because the noise was inside the frequency band), short record, and dead channel. The data are written in a format which are compatible with the standard format for the California StrongMotion Instrumentation Program (CSMIP) tapes [Reference 3]. Table 3 summaries the standard data format.

The data were written on a 8MM tape using ASCII format (record length $=80$, blocksize $=2000$ ). Table 4 gives examples of VAX/VMS commands on how the data tape was created and how the data files can be retrieved.

\section{Acknowledgment}

The network of seismographs in southern Nevada to monitor the ground motion generated by the UNEs at the NTS was maintained by the staff of electronic technicians consisting of Ray Bradley, Don Morgan, Robert Stewart, Lori Forrester and Jim Graham. The seismic records conversion and processing staff included Vickie Ross, Stephanie Miller, Rosemarie Musso, Ben Gacula and Cory Lieber.

\section{References}

1. Navarro, R., and G.M. Wuollet, "The L-7 Velocity Seismograph Shaking-Table Results", NOAA Technical Report ERL 254-ESL 26, Boulder, Colorado, July 1972.

2. Lum, P. K., "Overview of Seismic Signal Conversion and Processing Operations", URS/John A. Blume \& Associates, Engineers, San Francisco, JAB-10733-TM3, March 1991.

3. Shakal, A. F. and M. J. Huang, "Standard Tape Format for CSMIP Strong-Motion Data Tapes", California Department of Conservation, Division of Mines and Geology, Office of Strong Motion Studies, Report OSMS 85-03, December 1985. 


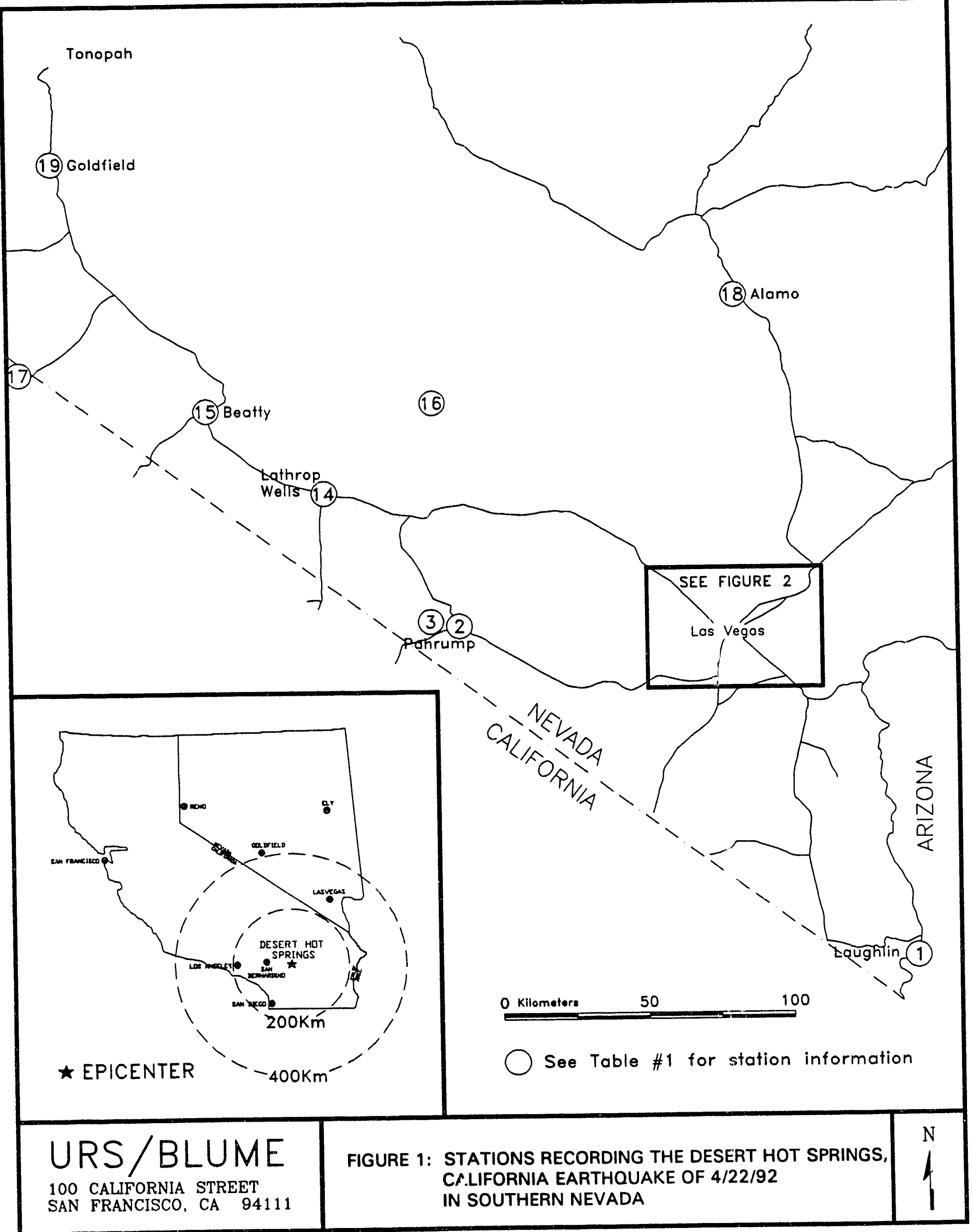




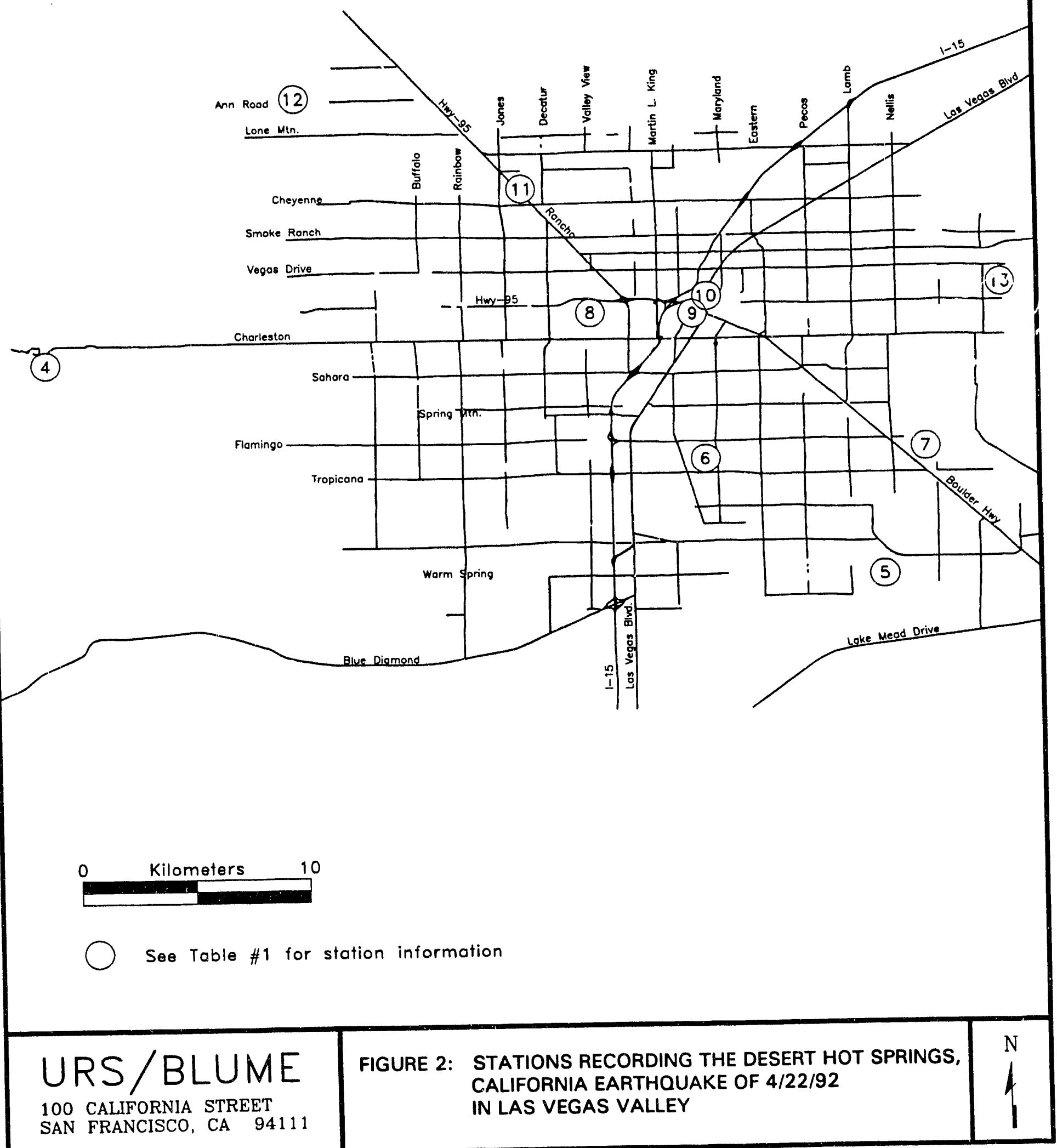


TABLE 1: LOCATIONS OF THE RECORDING STATIONS

(Desert Hot Springs, California Earthquake of April 22, 1992)

\begin{tabular}{|c|c|c|c|c|c|}
\hline \multirow{2}{*}{$\begin{array}{c}\begin{array}{c}\text { Station } \\
\text { No. }\end{array} \\
1\end{array}$} & \multirow{2}{*}{$\begin{array}{l}\text { Recording } \\
\text { System(8) } \\
\text { PDAS-100 }\end{array}$} & \multirow{2}{*}{$\begin{array}{l}\text { Location } \\
\text { Laughlin }\end{array}$} & \multicolumn{2}{|c|}{ Coordinates } & \multirow{2}{*}{$\begin{array}{l}\text { Distance from } \\
\text { Epicenter }(\mathrm{Km}) \\
209\end{array}$} \\
\hline & & & $114.58 W$ & $35.15 \mathrm{~N}$ & \\
\hline 2 & PDR -1 & Pahrump Site 1 & $115.98 \mathrm{~W}$ & $36.21 \mathrm{~N}$ & 254 \\
\hline 3 & PDAS-100 & Pahrurnp site 2 & $116.07 \mathrm{~W}$ & $36.23 \mathrm{~N}$ & 255 \\
\hline 4 & PDR-1 & Las Vegas & $115.41 \mathrm{~W}$ & $36.15 \mathrm{~N}$ & 259 \\
\hline 5 & PDR-1 & Las Vegas & $115.07 \mathrm{~W}$ & $36.06 \mathrm{~N}$ & 262 \\
\hline $\begin{array}{l}6 A \\
6 B\end{array}$ & $\begin{array}{l}L-7 \\
\text { PDR-1 }\end{array}$ & Las Vegas & $115.14 \mathrm{~W}$ & $36.11 \mathrm{~N}$ & 264 \\
\hline 7 & PDR-1 & Las Vegas & $115.05 \mathrm{~W}$ & $36.11 \mathrm{~N}$ & 268 \\
\hline $\begin{array}{l}8 \mathrm{~A} \\
8 \mathrm{~B}\end{array}$ & $\begin{array}{l}\text { PDR-1 } \\
\text { PDAS-100 }\end{array}$ & Las Vegas & $115.19 \mathrm{~W}$ & $36.17 \mathrm{~N}$ & 269 \\
\hline 9 & PDR-1 & Las Vegas & $115.14 \mathrm{~W}$ & $36.17 \mathrm{~N}$ & 270 \\
\hline 10 & PDR - 1 & Las Vegas & $115.14 \mathrm{~W}$ & $36.17 \mathrm{~N}$ & 271 \\
\hline 11 & PDR-1 & Las Vegas & $115.22 \mathrm{~W}$ & $36.22 \mathrm{~N}$ & 273 \\
\hline 12 & PDR-1 & Las Vegas & $115.31 \mathrm{~W}$ & $36.26 \mathrm{~N}$ & 274 \\
\hline 13 & PDR-1 & Las Vegas & $115.02 \mathrm{~W}$ & $36.18 \mathrm{~N}$ & 276 \\
\hline 14 & PDAS-100 & Lathrop Wells & $116.40 \mathrm{~W}$ & $36.64 \mathrm{~N}$ & 300 \\
\hline 15 & PDR-1 & Beatty & $316.76 \mathrm{~W}$ & $36.91 \mathrm{~N}$ & 332 \\
\hline 16 & $P D R-1$ & Nevada Test Site & $116.06 \mathrm{~W}$ & $36.93 N$ & 333 \\
\hline $\begin{array}{l}17 \mathrm{~A} \\
17 \mathrm{~B}\end{array}$ & $\begin{array}{l}\text { PDR-1 } \\
\text { PDAS-100 }\end{array}$ & Death Valley & $117.34 \mathrm{~W}$ & $37.03 N$ & 355 \\
\hline $\begin{array}{l}18 \mathrm{~A} \\
18 \mathrm{~B}\end{array}$ & $\begin{array}{l}\text { PDR-1 } \\
\text { PDAS-100 }\end{array}$ & Alamo & $115.12 \mathrm{~W}$ & $37.27 \mathrm{~N}$ & 385 \\
\hline 19 & PDAS -100 & Goldfield & $117.23 \mathrm{~W}$ & $37.71 \mathrm{~N}$ & 426 \\
\hline
\end{tabular}


TABLE 2: PEAK AMPLITUDES

(Desert Hot Springs, California Earthquake of April 22, 1992)

\begin{tabular}{|c|c|c|c|c|c|c|c|}
\hline $\begin{array}{l}\text { station } \\
\text { No. }\end{array}$ & Comp & $\begin{array}{l}<- \text { Uncorre } \\
\text { Accel } \\
\left(\mathrm{cm} / \mathrm{sec}^{2}\right)\end{array}$ & $\begin{array}{l}\text { ected } \rightarrow \\
\text { Velocity } \\
(\mathrm{cm} / \mathrm{sec})\end{array}$ & $\begin{array}{c}\text { Accel } \\
\left(\mathrm{cm} / \mathrm{sec}^{2}\right)\end{array}$ & $\begin{array}{c}\text { Corrected - } \\
\text { Velocity } \\
(\mathrm{cm} / \mathrm{sec})\end{array}$ & $\begin{array}{l}\text { Disp } \\
(\mathrm{cm})\end{array}$ & Comments \\
\hline $\begin{array}{l}1 \\
1 \\
1\end{array}$ & $\begin{array}{l}\text { DOWN } \\
\text { NORTH } \\
\text { WEST }\end{array}$ & $\begin{array}{r}4.907 \\
9.799 \\
-9.750\end{array}$ & $\begin{array}{l}0.000 \\
0.000 \\
0.000\end{array}$ & $\begin{array}{r}4.829 \\
9.838 \\
-9.811\end{array}$ & $\begin{array}{r}0.211 \\
0.381 \\
-0.499\end{array}$ & $\begin{array}{r}0.154 \\
-0.225 \\
-0.252\end{array}$ & \\
\hline $\begin{array}{l}2 \\
2 \\
2\end{array}$ & $\begin{array}{l}\text { DOWN } \\
\text { NORTH } \\
\text { WEST }\end{array}$ & $\begin{array}{r}2.079 \\
-0.015 \\
3.830\end{array}$ & $\begin{array}{l}0.000 \\
0.000 \\
0.000\end{array}$ & $\begin{array}{c}2.077 \\
-- \\
3.820\end{array}$ & $\begin{array}{l}0.238 \\
0.940\end{array}$ & $\begin{array}{c}-0.093 \\
-- \\
0.783\end{array}$ & DEAD CHANNEL \\
\hline $\begin{array}{l}3 \\
3 \\
3\end{array}$ & $\begin{array}{l}\text { DOWN } \\
\text { NORTH } \\
\text { WEST }\end{array}$ & $\begin{array}{l}3.700 \\
7.558 \\
8.145\end{array}$ & $\begin{array}{l}0.000 \\
0.000 \\
0.000\end{array}$ & $\begin{array}{l}3.697 \\
7.554 \\
8.139\end{array}$ & $\begin{array}{r}0.516 \\
-1.451 \\
1.959\end{array}$ & $\begin{array}{r}0.105 \\
-0.432 \\
0.904\end{array}$ & \\
\hline $\begin{array}{l}4 \\
4 \\
4\end{array}$ & $\begin{array}{l}\text { DOWN } \\
\text { NORTH } \\
\text { WEST }\end{array}$ & $\begin{array}{r}1.795 \\
-1.911 \\
2.593\end{array}$ & $\begin{array}{l}0.000 \\
0.000 \\
0.000\end{array}$ & $\begin{array}{r}1.791 \\
-1.911 \\
2.581\end{array}$ & $\begin{array}{l}-0.224 \\
-0.349 \\
-0.482\end{array}$ & $\begin{array}{l}-0.099 \\
-0.159 \\
-0.341\end{array}$ & \\
\hline $\begin{array}{l}5 \\
5 \\
5\end{array}$ & $\begin{array}{l}\text { DOWN } \\
\text { NORTH } \\
\text { WEST }\end{array}$ & $\begin{array}{r}2.767 \\
-3.104 \\
3.079\end{array}$ & $\begin{array}{l}0.000 \\
0.000 \\
0.000\end{array}$ & $\begin{array}{r}2.763 \\
-3.128 \\
3.067\end{array}$ & $\begin{array}{r}0.501 \\
0.479 \\
-0.717\end{array}$ & $\begin{array}{r}0.167 \\
0.202 \\
-0.430\end{array}$ & \\
\hline $\begin{array}{ll}6 & A \\
6 & A \\
6 & A\end{array}$ & $\begin{array}{l}\text { DOWN } \\
\text { NORTH } \\
\text { WEST }\end{array}$ & $\begin{array}{l}0.000 \\
0.000 \\
0.000\end{array}$ & $\begin{array}{r}0.345 \\
-0.646 \\
-0.671\end{array}$ & $\begin{array}{r}2.909 \\
-3.606 \\
-4.105\end{array}$ & $\begin{array}{r}0.347 \\
-0.635 \\
-0.662\end{array}$ & $\begin{array}{l}0.105 \\
0.234 \\
0.372\end{array}$ & NOISY RECORDS \\
\hline $\begin{array}{ll}6 & B \\
6 & B \\
6 & B\end{array}$ & $\begin{array}{l}\text { DOWN } \\
\text { NORTH } \\
\text { WEST }\end{array}$ & $\begin{array}{r}2.055 \\
-3.522 \\
-3.495\end{array}$ & $\begin{array}{l}0.000 \\
0.000 \\
0.000\end{array}$ & $\begin{array}{r}2.035 \\
-3.520 \\
-3.446\end{array}$ & $\begin{array}{l}0.352 \\
0.732 \\
1.094\end{array}$ & $\begin{array}{l}-0.120 \\
-0.335 \\
-0.388\end{array}$ & \\
\hline $\begin{array}{l}7 \\
7 \\
7\end{array}$ & $\begin{array}{l}\text { DOWN } \\
\text { NORTH } \\
\text { WEST }\end{array}$ & $\begin{array}{r}-1.835 \\
-2.714 \\
3.709\end{array}$ & $\begin{array}{l}0.000 \\
0.000 \\
0.000\end{array}$ & $\begin{array}{r}-1.825 \\
-2.707 \\
3.709\end{array}$ & $\begin{array}{l}-0.399 \\
-0.521 \\
-0.725\end{array}$ & $\begin{array}{r}-0.125 \\
0.209 \\
-0.389\end{array}$ & \\
\hline $\begin{array}{ll}8 & A \\
8 & A \\
8 & A\end{array}$ & $\begin{array}{l}\text { DOWN } \\
\text { NORTH } \\
\text { WEST }\end{array}$ & $\begin{array}{r}2.321 \\
0.210 \\
-7.024\end{array}$ & $\begin{array}{l}0.000 \\
0.000 \\
0.000\end{array}$ & $\begin{array}{c}2.296 \\
-7.034\end{array}$ & $\begin{array}{c}-0.445 \\
- \\
-1.380\end{array}$ & $\begin{array}{c}-0.114 \\
-- \\
0.657\end{array}$ & DEAD CHANNEL \\
\hline $\begin{array}{ll}8 & B \\
8 & B \\
8 & B\end{array}$ & $\begin{array}{l}\text { DOWN } \\
\text { NORTH } \\
\text { WEST }\end{array}$ & $\begin{array}{r}2.311 \\
-5.975 \\
-7.116\end{array}$ & $\begin{array}{l}0.000 \\
0.000 \\
0.000\end{array}$ & $\begin{array}{r}2.313 \\
-5.968 \\
-7.114\end{array}$ & $\begin{array}{r}-0.442 \\
1.329 \\
-1.378\end{array}$ & $\begin{array}{l}0.110 \\
0.533 \\
0.679\end{array}$ & \\
\hline $\begin{array}{l}9 \\
9 \\
9\end{array}$ & $\begin{array}{l}\text { DOWN } \\
\text { NORTH } \\
\text { WEST }\end{array}$ & $\begin{array}{r}4.180 \\
4.936 \\
-6.859\end{array}$ & $\begin{array}{l}0.000 \\
0.000 \\
0.000\end{array}$ & $\begin{array}{r}4.144 \\
4.940 \\
-6.841\end{array}$ & $\begin{array}{l}0.884 \\
1.153 \\
1.847\end{array}$ & $\begin{array}{r}-0.205 \\
-0.342 \\
0.807\end{array}$ & \\
\hline $\begin{array}{l}10 \\
10 \\
10\end{array}$ & $\begin{array}{l}\text { DOWN } \\
\text { NORTH } \\
\text { WEST }\end{array}$ & $\begin{array}{r}-2.409 \\
5.708 \\
-6.513\end{array}$ & $\begin{array}{l}0.000 \\
0.000 \\
0.000\end{array}$ & $\begin{array}{r}-2.402 \\
5.706 \\
-6.525\end{array}$ & $\begin{array}{l}0.565 \\
1.384 \\
1.634\end{array}$ & $\begin{array}{r}0.194 \\
-0.452 \\
0.843\end{array}$ & \\
\hline $\begin{array}{l}11 \\
11 \\
11\end{array}$ & $\begin{array}{l}\text { DOWN } \\
\text { NORTH } \\
\text { WEST }\end{array}$ & $\begin{array}{r}1.873 \\
-4.412 \\
-4.003\end{array}$ & $\begin{array}{l}0.000 \\
0.000 \\
0.000\end{array}$ & $\begin{array}{r}1.846 \\
-4.415 \\
-3.996\end{array}$ & $\begin{array}{r}0.262 \\
-0.639 \\
0.742\end{array}$ & $\begin{array}{r}0.139 \\
-0.263 \\
-0.454\end{array}$ & \\
\hline $\begin{array}{l}12 \\
12 \\
12\end{array}$ & $\begin{array}{l}\text { DOWN } \\
\text { NORTH } \\
\text { WEST }\end{array}$ & $\begin{array}{l}1.556 \\
2.022 \\
1.947\end{array}$ & $\begin{array}{l}0.000 \\
0.000 \\
0.000\end{array}$ & $\begin{array}{l}1.552 \\
2.021 \\
1.938\end{array}$ & $\begin{array}{l}0.279 \\
0.505 \\
0.594\end{array}$ & $\begin{array}{l}-0.127 \\
-0.244 \\
-0.457\end{array}$ & \\
\hline
\end{tabular}




\section{TABLE 2: PEAK AMPLITUDES (CONT'D)}

\begin{tabular}{|c|c|c|c|c|c|c|c|}
\hline $\begin{array}{l}\text { Station } \\
\text { No. }\end{array}$ & Comp & $\begin{array}{l}<- \text { Uncorre } \\
\text { Accel } \\
\left(\mathrm{cm} / \mathrm{sec}^{2}\right)\end{array}$ & $\begin{array}{l}\text { ected } \rightarrow \\
\text { Velocity } \\
(\mathrm{cm} / \mathrm{sec})\end{array}$ & $\begin{array}{c}\text { Accel } \\
\left(\mathrm{cm} / \mathrm{sec}^{2}\right)\end{array}$ & $\begin{array}{c}\text { Corrected } \\
\text { Velocity } \\
(\mathrm{cm} / \mathrm{sec})\end{array}$ & $\begin{array}{c}\text { Disp } \\
(\mathrm{cm})\end{array}$ & Comments \\
\hline $\begin{array}{l}13 \\
13 \\
13\end{array}$ & $\begin{array}{l}\text { DOWN } \\
\text { NORTH } \\
\text { WEST }\end{array}$ & $\begin{array}{r}-0.769 \\
1.002 \\
1.542\end{array}$ & $\begin{array}{l}0.000 \\
0.000 \\
0.000\end{array}$ & $\begin{array}{r}-0.759 \\
0.966 \\
1.519\end{array}$ & $\begin{array}{r}-0.115 \\
0.227 \\
0.249\end{array}$ & $\begin{array}{l}0.090 \\
0.145 \\
0.198\end{array}$ & \\
\hline $\begin{array}{l}14 \\
14 \\
14\end{array}$ & $\begin{array}{l}\text { DOWN } \\
\text { NORTH } \\
\text { WEST }\end{array}$ & $\begin{array}{l}-1.383 \\
-4.011 \\
-3.347\end{array}$ & $\begin{array}{l}0.000 \\
0.000 \\
0.000\end{array}$ & $\begin{array}{l}-1.374 \\
-4.003 \\
-3.350\end{array}$ & $\begin{array}{r}-0.313 \\
0.623 \\
-0.837\end{array}$ & $\begin{array}{r}-0.151 \\
-0.234 \\
0.806\end{array}$ & \\
\hline $\begin{array}{l}15 \\
15 \\
15\end{array}$ & $\begin{array}{l}\text { DOWN } \\
\text { NORTH } \\
\text { WEST }\end{array}$ & $\begin{array}{r}1.855 \\
-2.335 \\
4.233\end{array}$ & $\begin{array}{l}0.000 \\
0.000 \\
0.000\end{array}$ & $\begin{array}{r}1.838 \\
-2.330 \\
4.225\end{array}$ & $\begin{array}{r}0.179 \\
0.361 \\
-0.403\end{array}$ & $\begin{array}{r}-0.089 \\
-0.147 \\
0.353\end{array}$ & \\
\hline $\begin{array}{l}16 \\
16 \\
16\end{array}$ & $\begin{array}{l}\text { DOWN } \\
\text { NORTH } \\
\text { WEST }\end{array}$ & $\begin{array}{r}-1.021 \\
1.161 \\
1.924\end{array}$ & $\begin{array}{l}0.000 \\
0.000 \\
0.000\end{array}$ & $\begin{array}{r}-1.010 \\
1.158 \\
1.923\end{array}$ & $\begin{array}{r}-0.203 \\
0.303 \\
-0.350\end{array}$ & $\begin{array}{r}-0.105 \\
0.164 \\
0.362\end{array}$ & $\begin{array}{l}\text { LATE TRIGGERED } \\
\text { SHORT RECORDS }\end{array}$ \\
\hline $\begin{array}{ll}17 & A \\
17 & A \\
17 & A\end{array}$ & $\begin{array}{l}\text { DOWN } \\
\text { NORTH } \\
\text { WEST }\end{array}$ & $\begin{array}{r}-0.697 \\
-1.500 \\
1.492\end{array}$ & $\begin{array}{l}0.000 \\
0.000 \\
0.000\end{array}$ & $\begin{array}{r}-0.691 \\
-1.496 \\
1.489\end{array}$ & $\begin{array}{r}-0.106 \\
-0.229 \\
0.356\end{array}$ & $\begin{array}{r}-0.086 \\
0.171 \\
-0.408\end{array}$ & $\begin{array}{l}\text { LATE TRIGGERED } \\
\text { SHORT RECORDS }\end{array}$ \\
\hline $\begin{array}{ll}17 & \mathrm{~B} \\
17 & \mathrm{~B} \\
17 & \mathrm{~B}\end{array}$ & $\begin{array}{l}\text { DOWN } \\
\text { NORTH } \\
\text { WEST }\end{array}$ & $\begin{array}{r}-0.685 \\
-1.498 \\
1.465\end{array}$ & $\begin{array}{l}0.000 \\
0.000 \\
0.000\end{array}$ & $\begin{array}{r}-0.669 \\
-1.497 \\
1.463\end{array}$ & $\begin{array}{r}-0.106 \\
-0.228 \\
0.360\end{array}$ & $\begin{array}{r}-0.080 \\
0.169 \\
-0.406\end{array}$ & \\
\hline $\begin{array}{ll}18 & A \\
18 & A \\
18 & A\end{array}$ & $\begin{array}{l}\text { DOWN } \\
\text { NORTH } \\
\text { WEST }\end{array}$ & $\begin{array}{r}1.847 \\
-3.167 \\
-2.805\end{array}$ & $\begin{array}{l}0.000 \\
0.000 \\
0.000\end{array}$ & $\begin{array}{r}1.187 \\
-2.403 \\
-2.591\end{array}$ & $\begin{array}{l}0.262 \\
0.465 \\
0.595\end{array}$ & $\begin{array}{r}0.093 \\
-0.229 \\
-0.386\end{array}$ & $\begin{array}{l}\text { LATE TRIGGERED } \\
\text { NOISY RECORDS }\end{array}$ \\
\hline $\begin{array}{ll}18 & B \\
18 & B \\
18 & B\end{array}$ & $\begin{array}{l}\text { DOWN } \\
\text { NORTH } \\
\text { WEST }\end{array}$ & $\begin{array}{l}0.120 \\
0.143 \\
0.231\end{array}$ & $\begin{array}{l}0.000 \\
0.000 \\
0.000\end{array}$ & $\begin{array}{l}0.098 \\
0.132 \\
0.155\end{array}$ & $\begin{array}{r}-0.036 \\
0.065 \\
0.058\end{array}$ & $\begin{array}{r}0.015 \\
-0.035 \\
-0.028\end{array}$ & LATE TRIGGERED \\
\hline $\begin{array}{l}19 \\
19 \\
19\end{array}$ & $\begin{array}{l}\text { DOWN } \\
\text { NORTH } \\
\text { WEST }\end{array}$ & $\begin{array}{r}-0.368 \\
-1.003 \\
0.865\end{array}$ & $\begin{array}{l}0.000 \\
0.000 \\
0.000\end{array}$ & $\begin{array}{r}-0.362 \\
-1.000 \\
0.859\end{array}$ & $\begin{array}{r}0.102 \\
-0.339 \\
-0.219\end{array}$ & $\begin{array}{l}-0.089 \\
-0.373 \\
-0.138\end{array}$ & \\
\hline
\end{tabular}




\section{TABLE 3: DATA FORMAT}

Each station contains 3 data channels and contains the following 4 files:

$\begin{array}{lll}\text { Vol } 1 & \text { Uncorrected acceleration } & \text { Units } \\ \text { Vol } 2 \text { Corrected acceleration } & \mathrm{cm} * 100 / \mathrm{gec} \\ & \begin{array}{l}\text { Corrected velocity } \\ \text { Corrected displacement }\end{array} & \mathrm{cm} * 100 / \mathrm{sec} \\ \text { Vol } 3 \text { Pseudo Response Velocity } & \mathrm{cm} / \mathrm{sec} \\ \text { Vol } 4 \text { Fourier Amplitude spectra } & \mathrm{cm} / \mathrm{sec}\end{array}$

A typical station contains the following data:

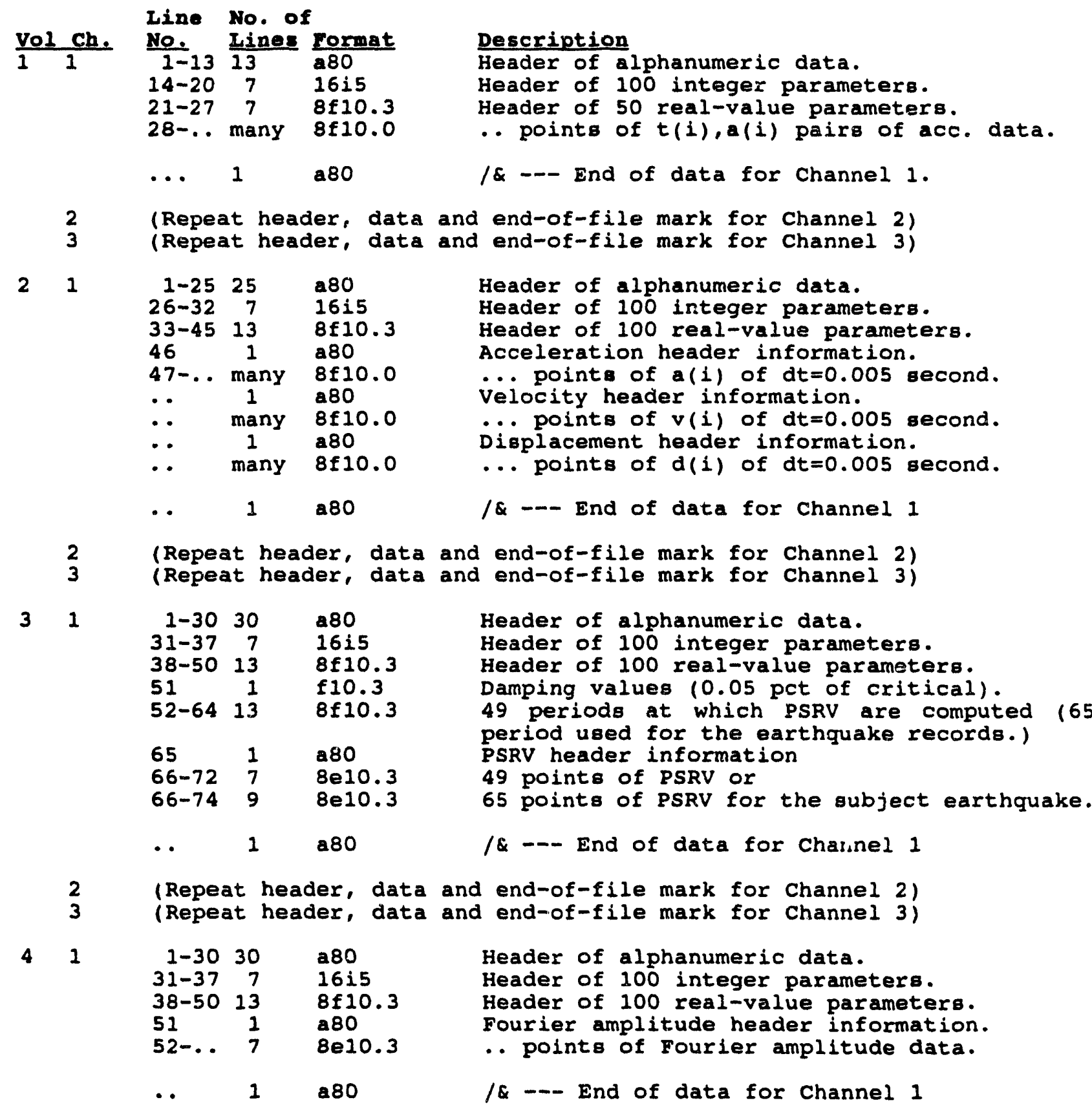

2 (Repeat header, data and end-of-file mark for Channel 2)

3 (Repeat header, data and end-of-file mark for Channel 3 ) 
TABLE 4: READ / WRITE DATA ON 8MM ASCII FORMATTED TAPE

WRITE DATA ON ASCII FORMATTED BMA TAPE

Below is an example to save files on $8 M$ M ASCII formatted tape with record length $=80$, block size $=2000$ using the VAx/VMS operating system utilities TWRITE:

\$ INIT MUBO: 0705B

$\$$ MOUNT/NOASSIST/FOR/RECORD $=80 / \mathrm{BLOCK}=2000$ MUBO:

$\$$ TWRITE/ASCII/RECORD $=80 /$ BLOCK $=2000 /$ OUTPUT $=M U B O$ :

\$ TWRITE/ASCII/RECORD $=80 /$ BLOCK $=2000 /$ OUTPUUT $=M U B O$ :

\$ TWRITE/ASCII / RECORD $=80 / \mathrm{BLOCK}=2000 /$ OUTPUT $=$ MUBO:

$\$$ TWRITE/ASCII / RECORD $=80 /$ BLOCK $=2000 /$ OUTPUT $=M U B O$ :

\$ TWRITE/ASCII / RECORD $=80 / \mathrm{BLOCK}=2000 /$ OUTPUT $=$ MUBO:

$\$$ TWRITE/ASCII/RECORD $=80 / \mathrm{BLOCK}=2000 /$ OUTPUT $=$ MUBO:

$\$$ TWRITE/ASCII /RECORD $=80 /$ BLOCK $=2000 /$ OUTPU' $\Gamma=M U B O$ :

\$ TWRITE/ASCII/RECORD $=80 /$ BLOCK $=2000 /$ OUTPUT $=$ MUBO:

STATN1.V1

STATN1.V2

STATN1.V3

STATN1.V4

STATN2.V1

STATN2.V2

STATN2.V3

STATN2.V4

... etc.. (for all stations, each with $v 1, v 2, v 3$ and v4 data)

\$ EXIT

READ/RETRIEVE DATA FROM ASCII FORMATIED 8MA TAPE

Below is an example to read/retrieve files from an 8MM ASCII formatted tape with record length $=80$, block size $=2000$ using the VAX/VMS operating system utilities TREAD:

\$ MOUNT/NOASSIST / FOR/RECORD $=80 / \mathrm{BLOCK}=2000$ MUBO:

$\$ T R E A D / A S C I I / R E C O R D=80 / B L O C K=2000 /$ INPUT=MUBO: STATNI.VI

$\$$ TREAD /ASCII /RECORD $=80 /$ BLOCK $=2000 /$ INPUT $=$ MUBO:

$\$ T R E A D / A S C I I / R E C O R D=80 / B L O C K=2000 / I N P U T=M U B O$ :

$\$$ TREAD $/$ ASCII $/$ RECORD $=80 / B L O C K=2000 /$ INPUT $=$ MUBO :

$\$ T R E A D / A S C I I / R E C O R D=80 / B L O C K=2000 /$ INPUT $=M U B O:$

\$ TREAD $/$ ASCII $/$ RECORD $=80 /$ BLOCK $=2000 /$ INPUT $=$ MUBO :

\$ TREAD/ASCII / RECORD $=80 / \mathrm{BLOCK}=2000 /$ INPUT $=$ MUBO:

$\$ T R E A D / A S C I I / R E C O R D=80 / B L O C K=2000 / I N P U T=M U B O$ :

STATN1.V2

STATN1.V3

STATN1.V4

STATN2.V1

STATN2.V2

STATN2.V3

... etc.. (for all otations, each with v1,v2,v3 and v4 data)

S EXIT 


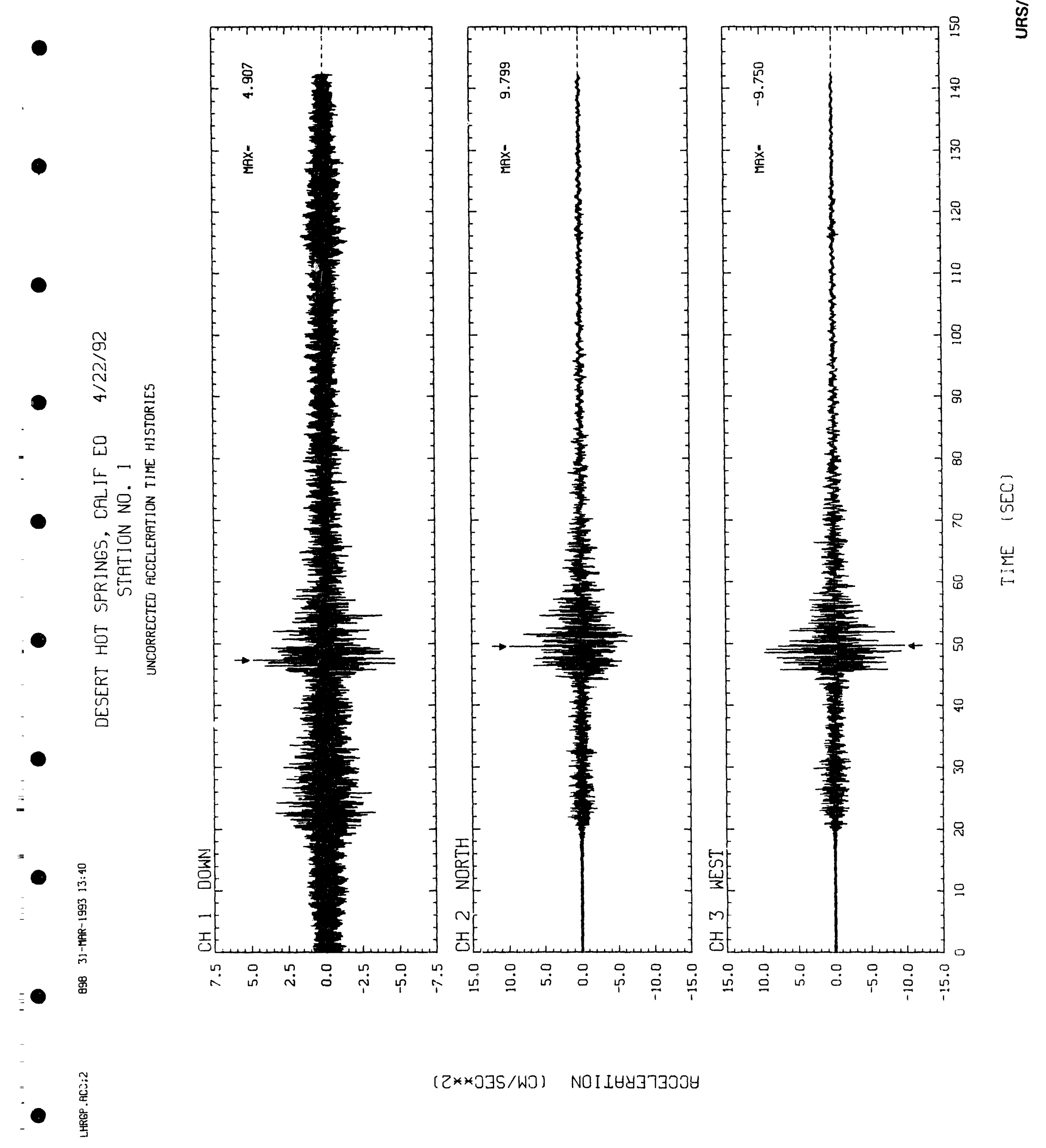




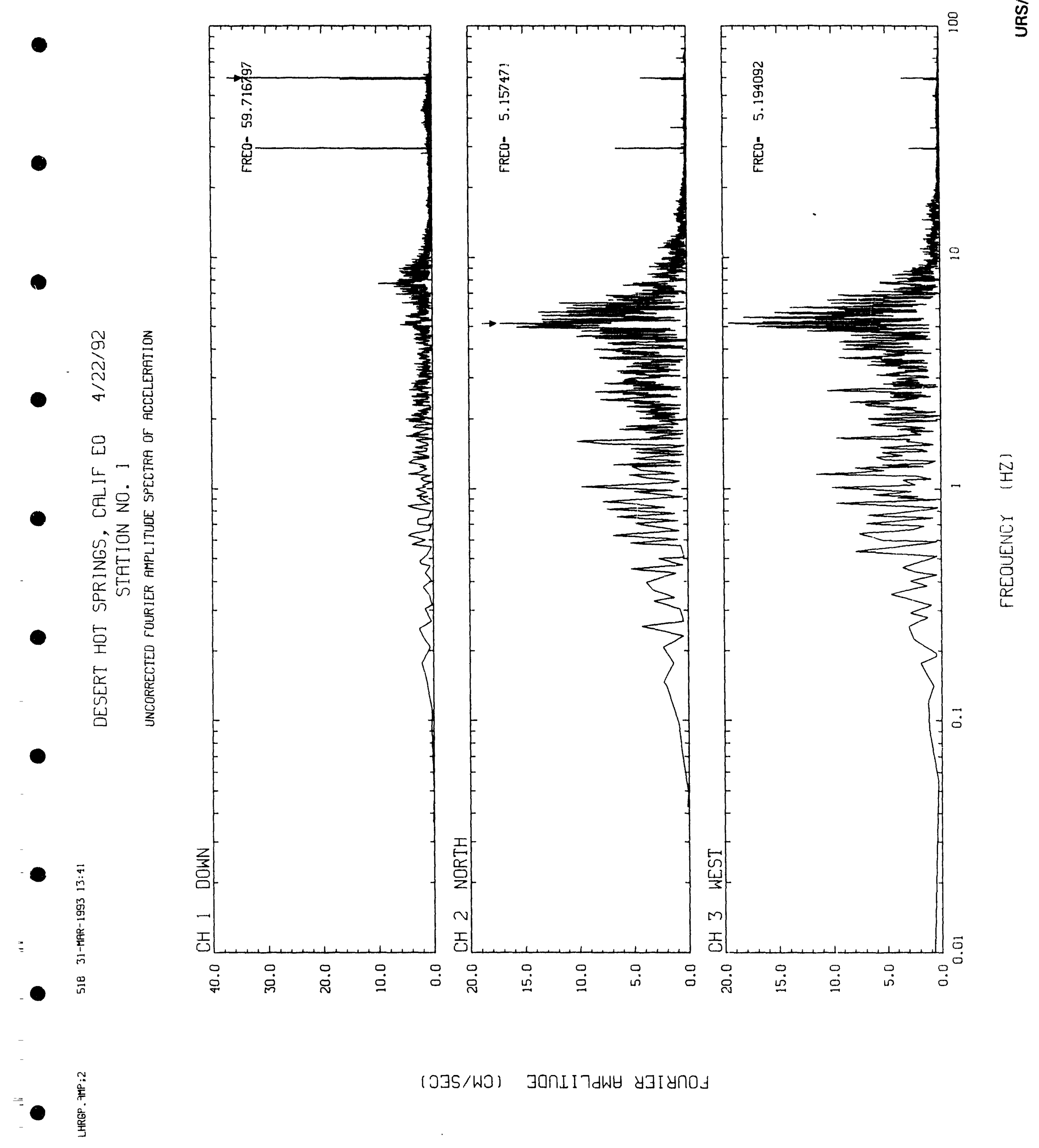




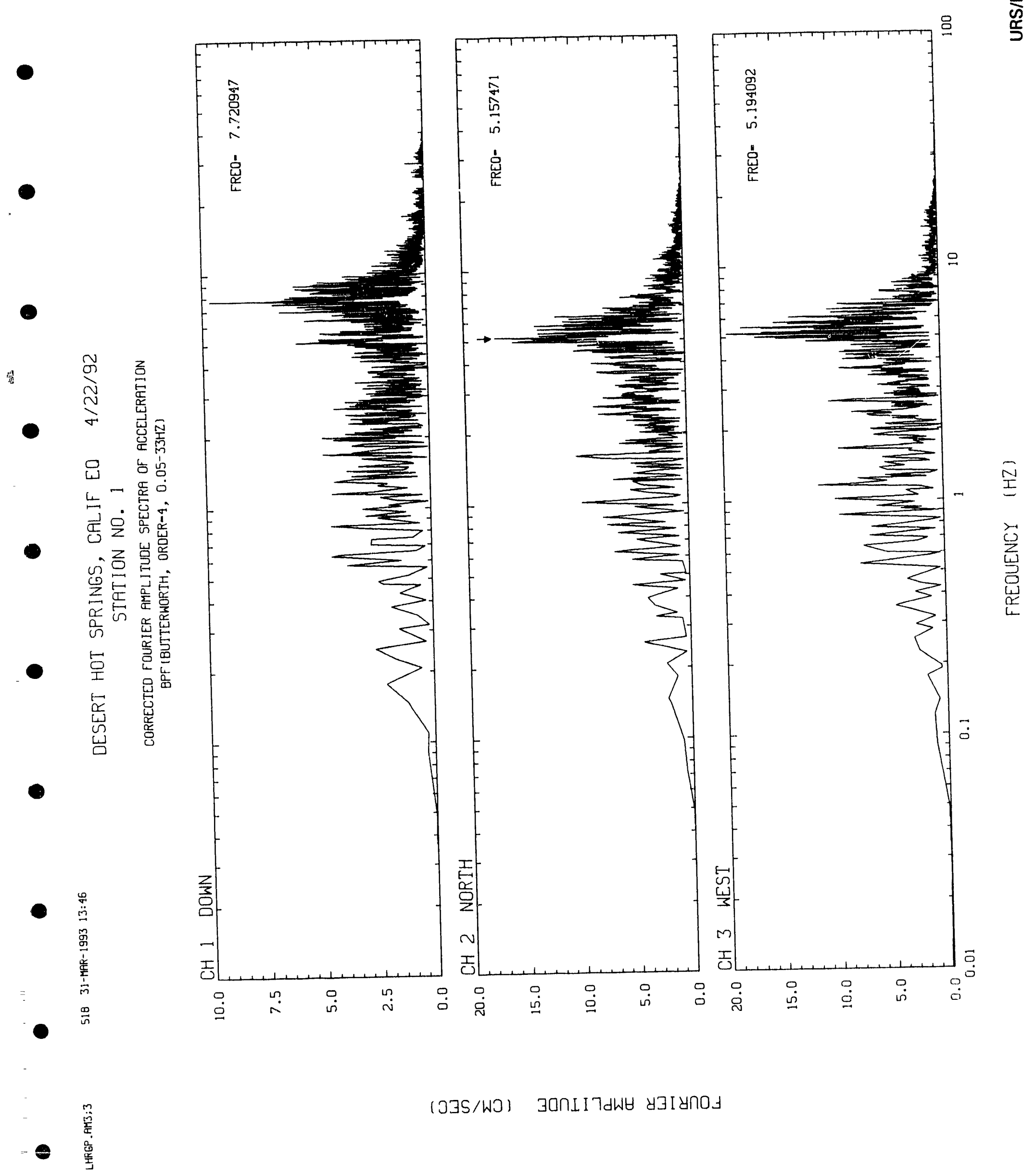




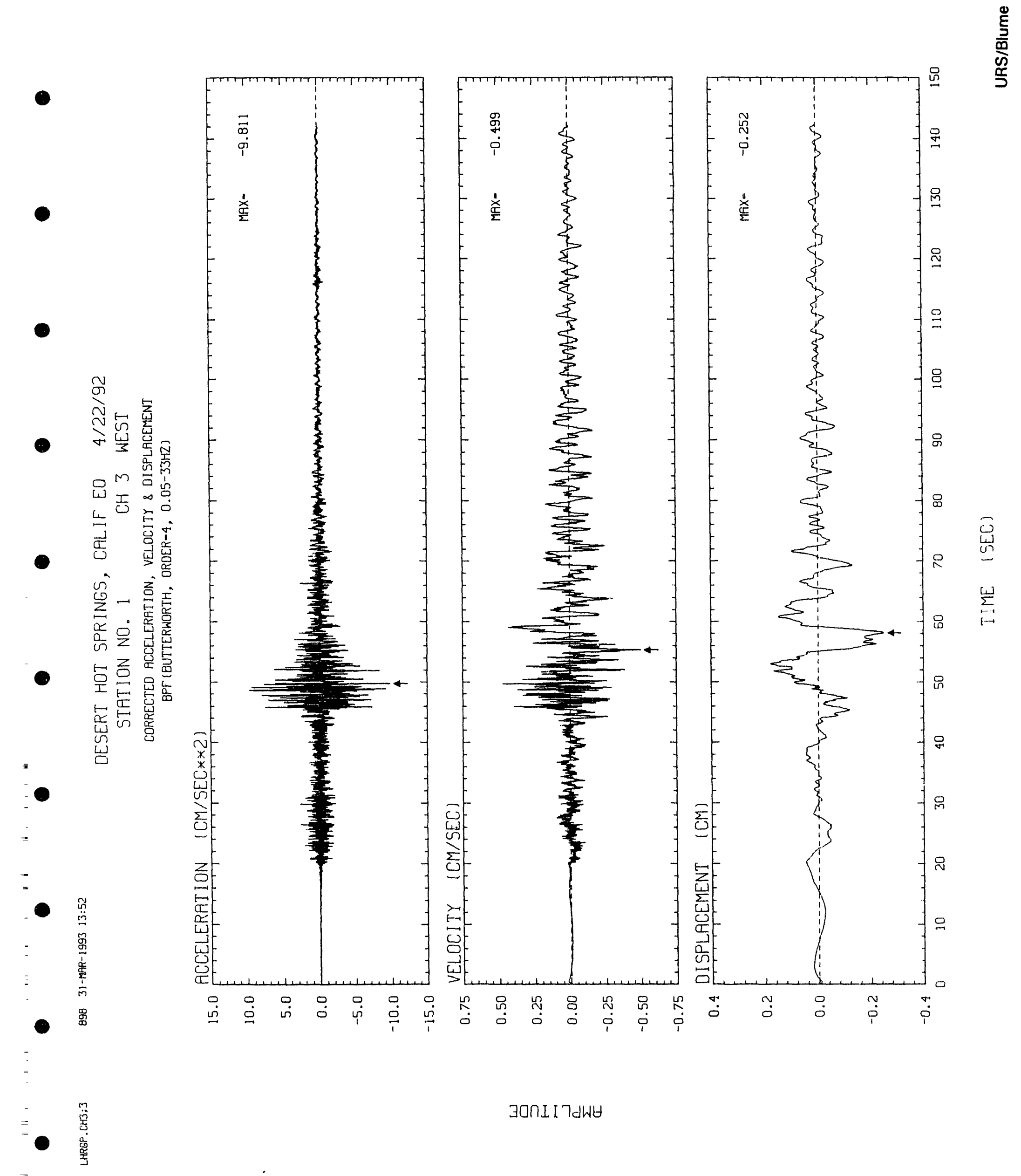



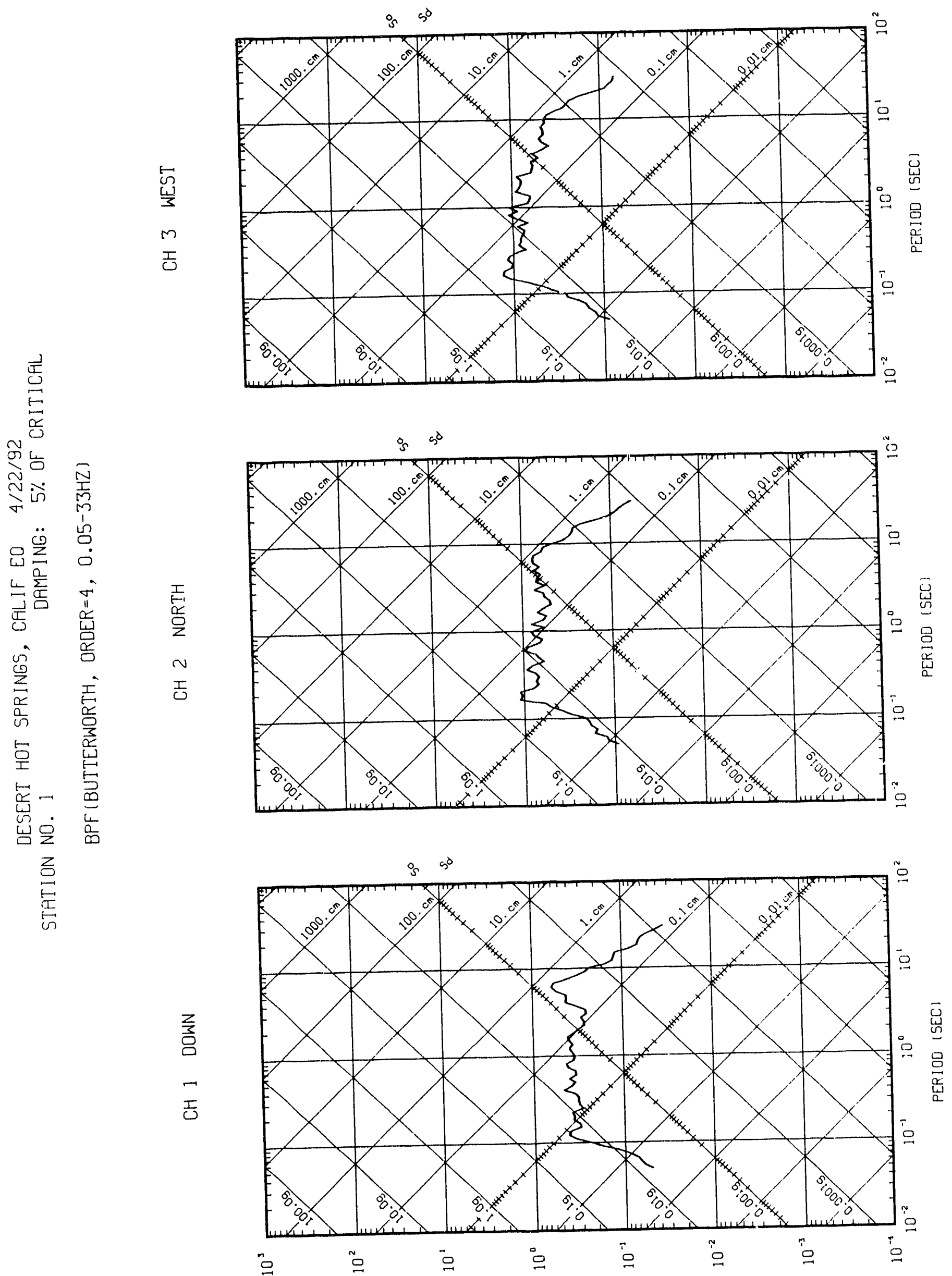
○

0

-

0

N

ำ

O

选 e 

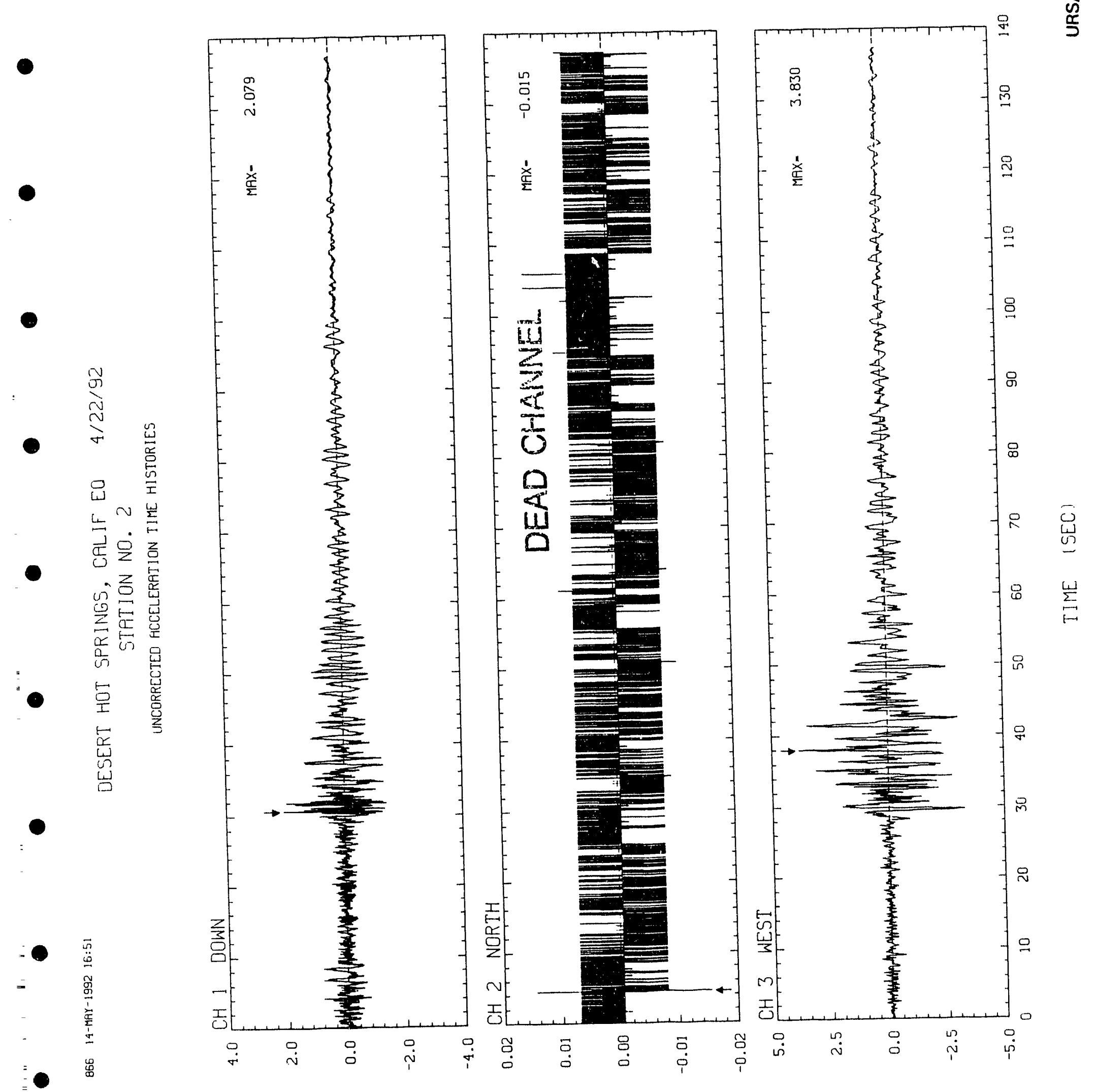

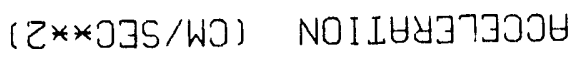




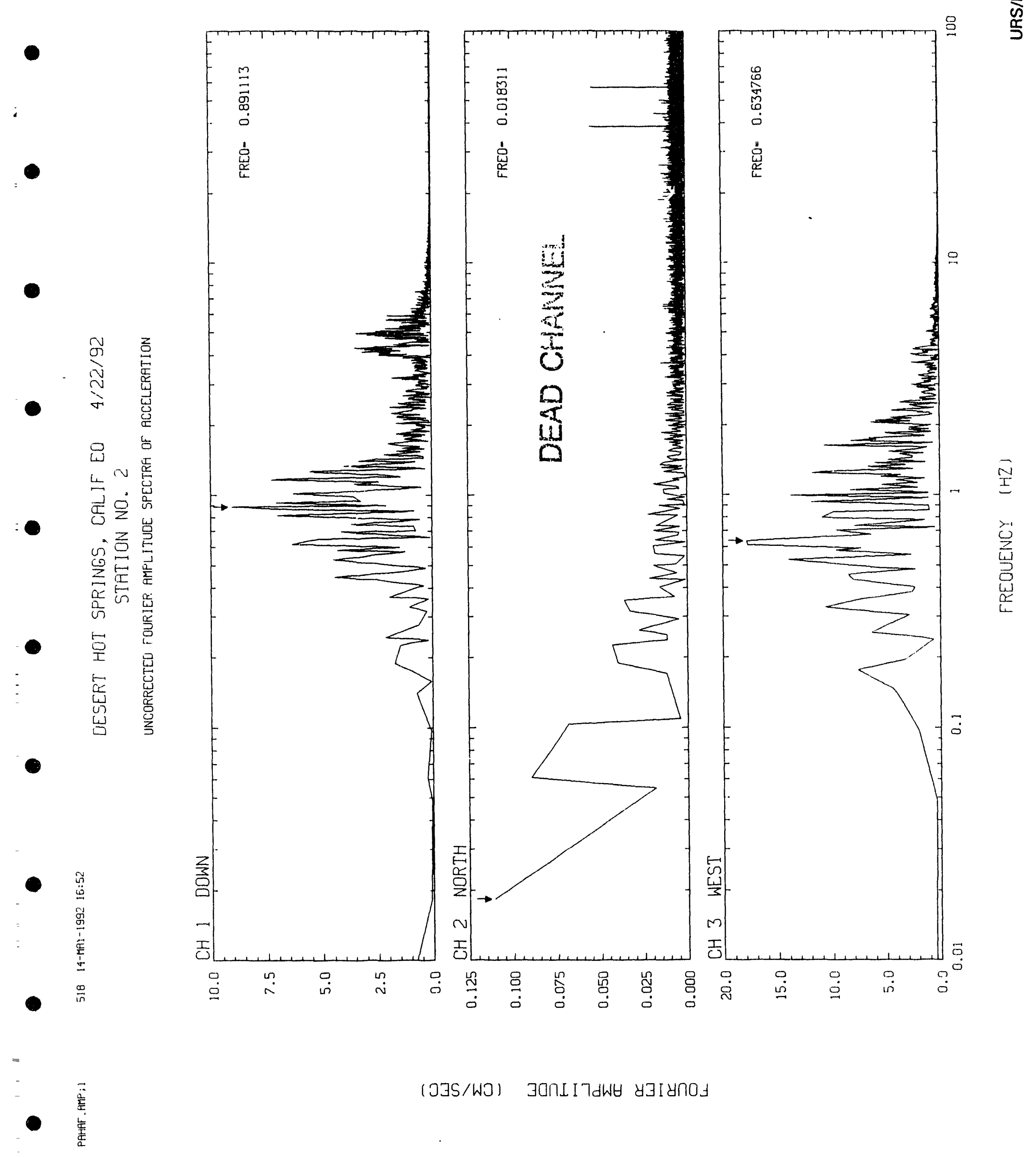



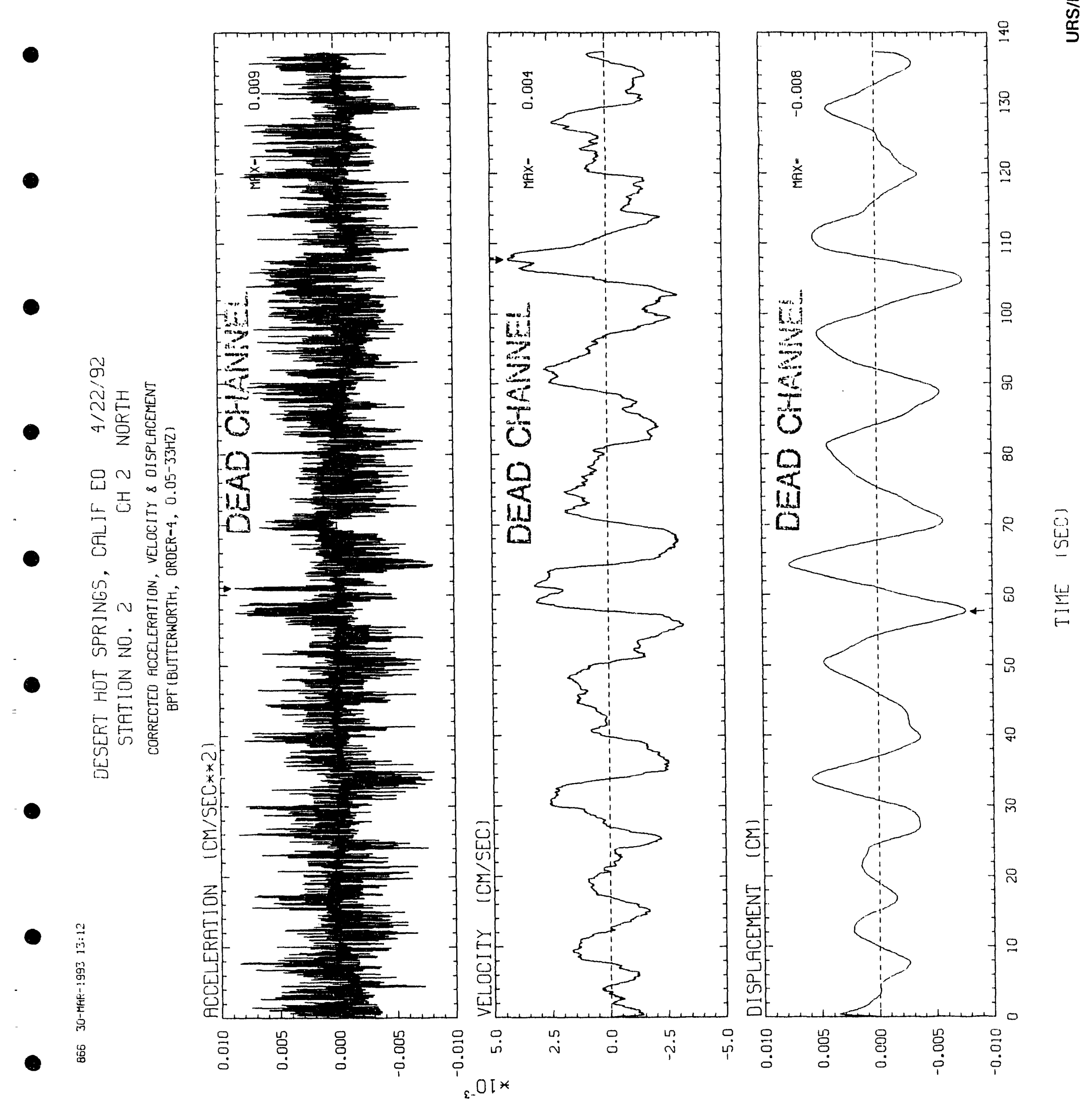


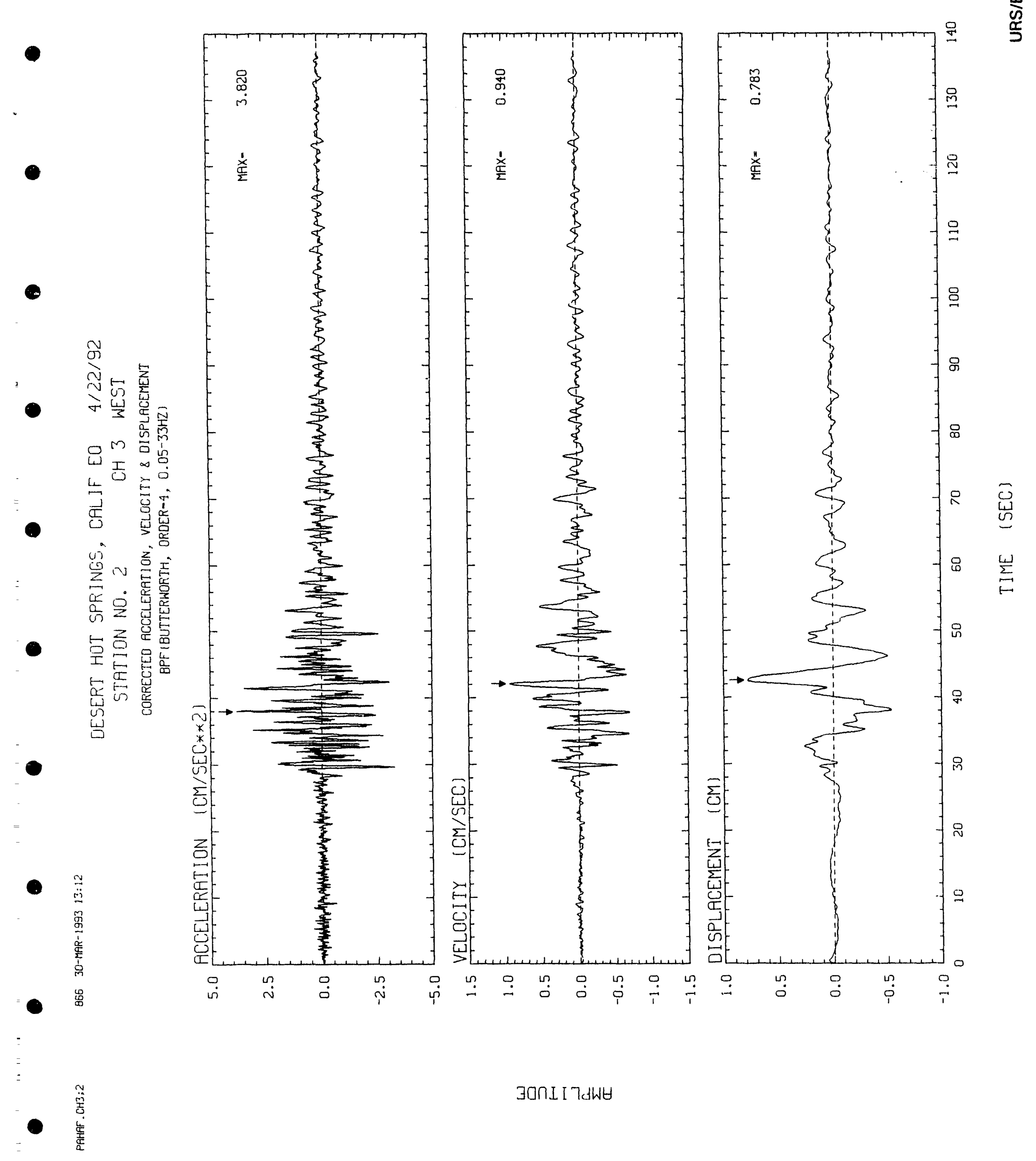




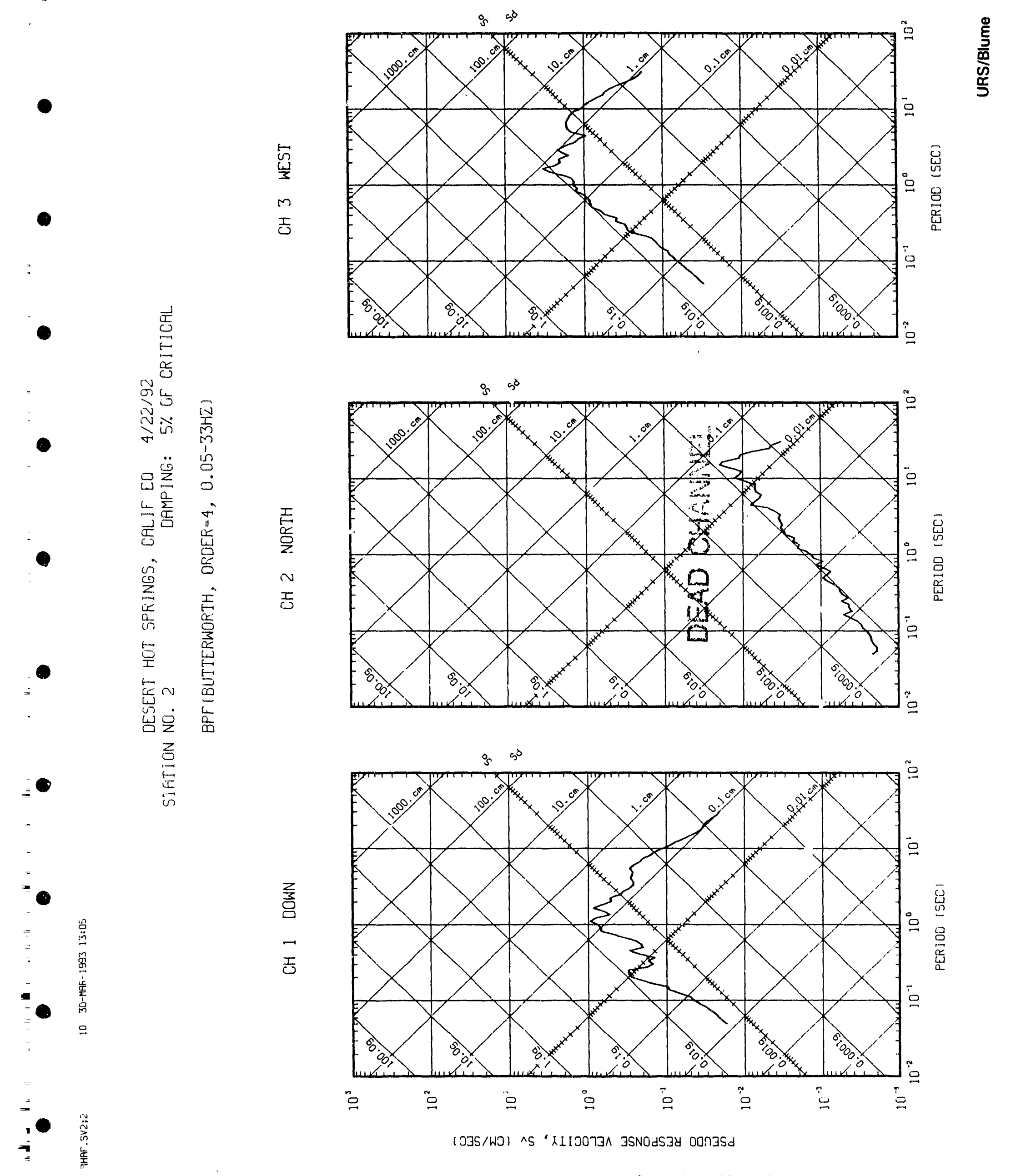


○

-

-

.

$m$

을

Z

选 乐

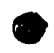

.

a

○ 


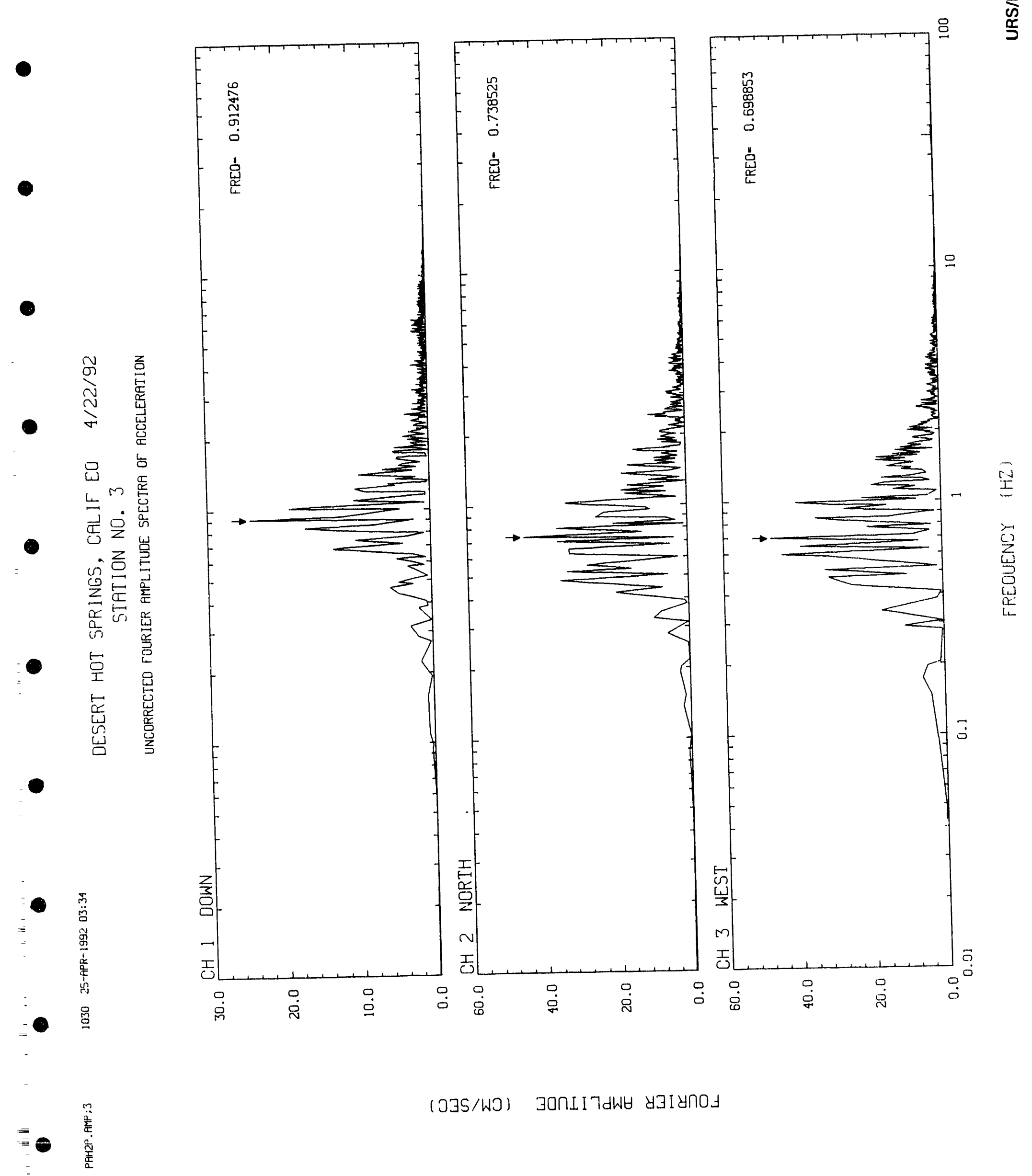



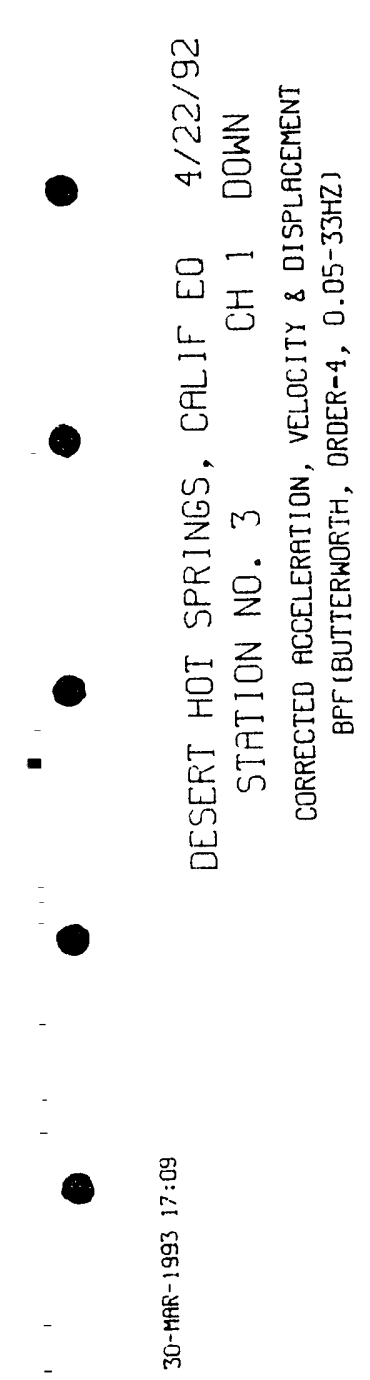

- $\stackrel{+}{a}$
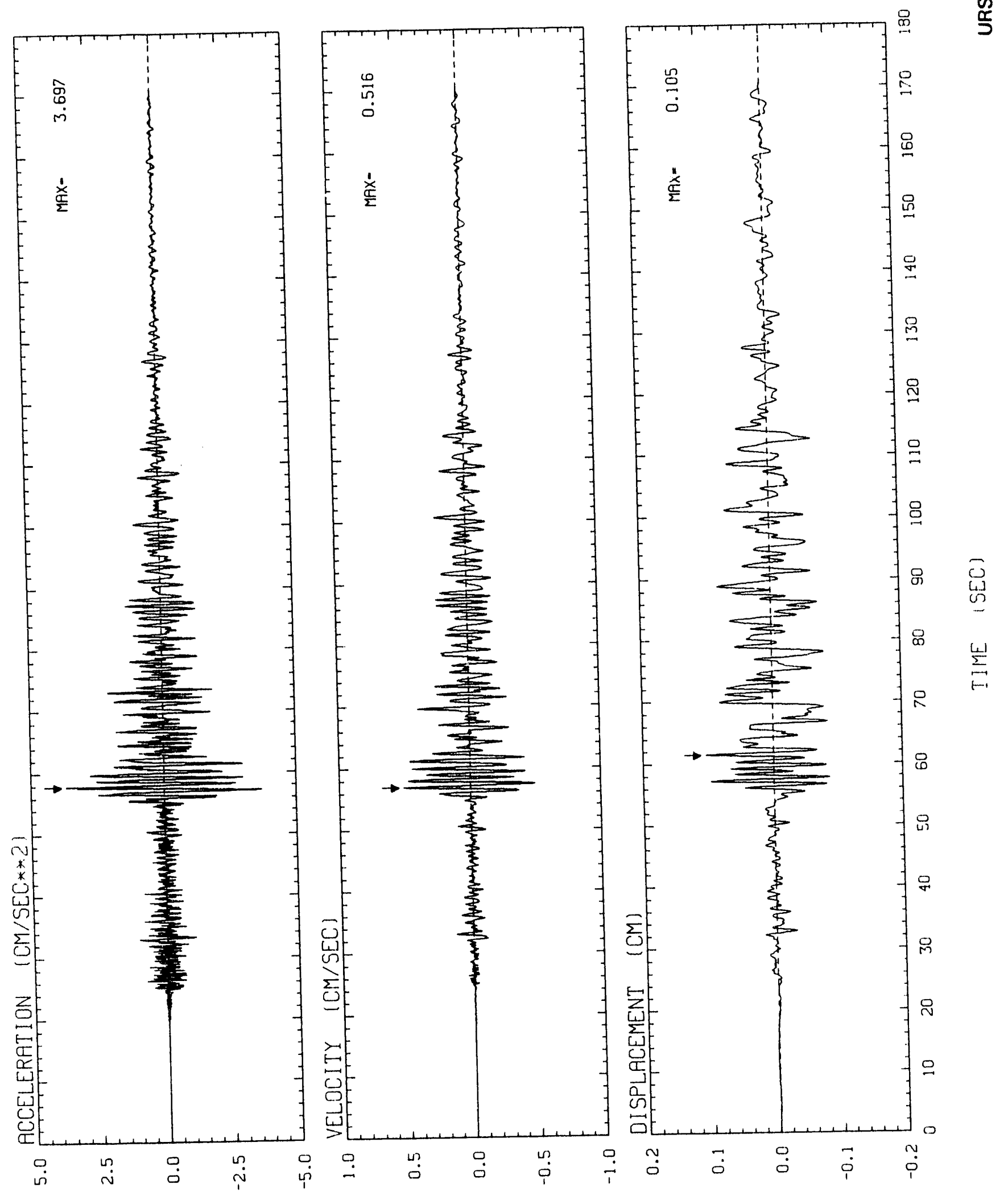

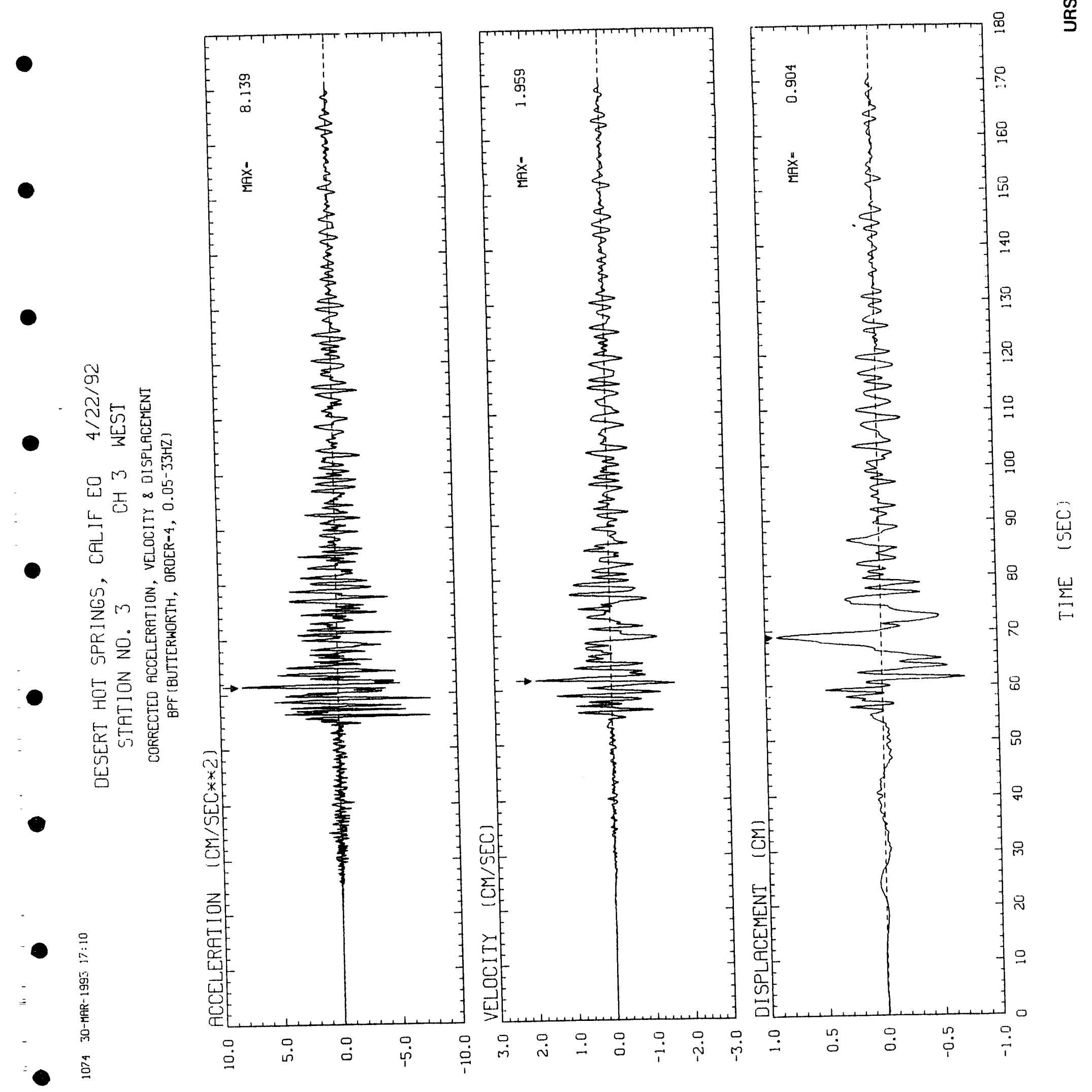

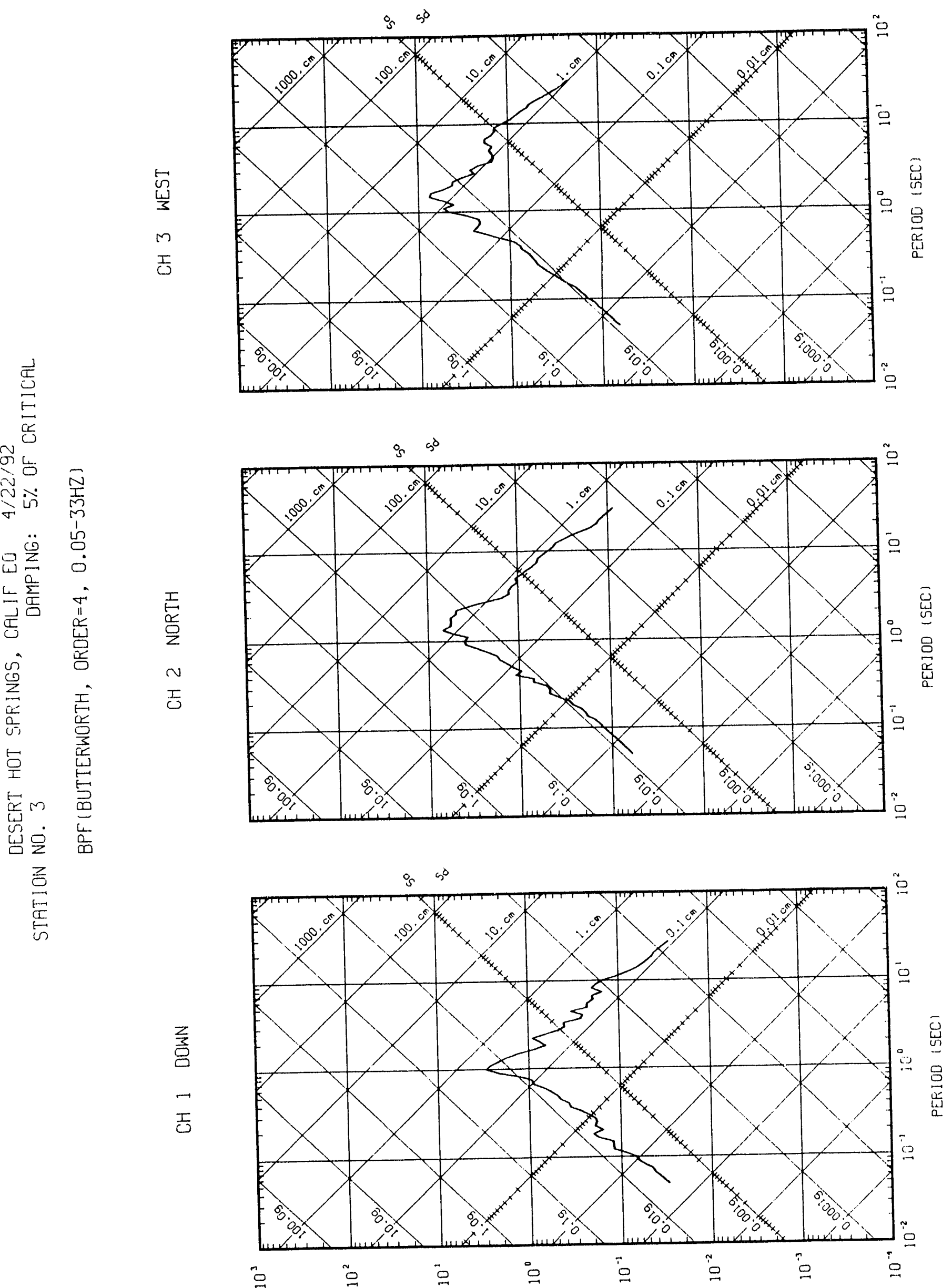

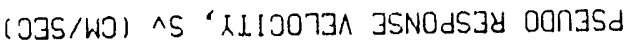




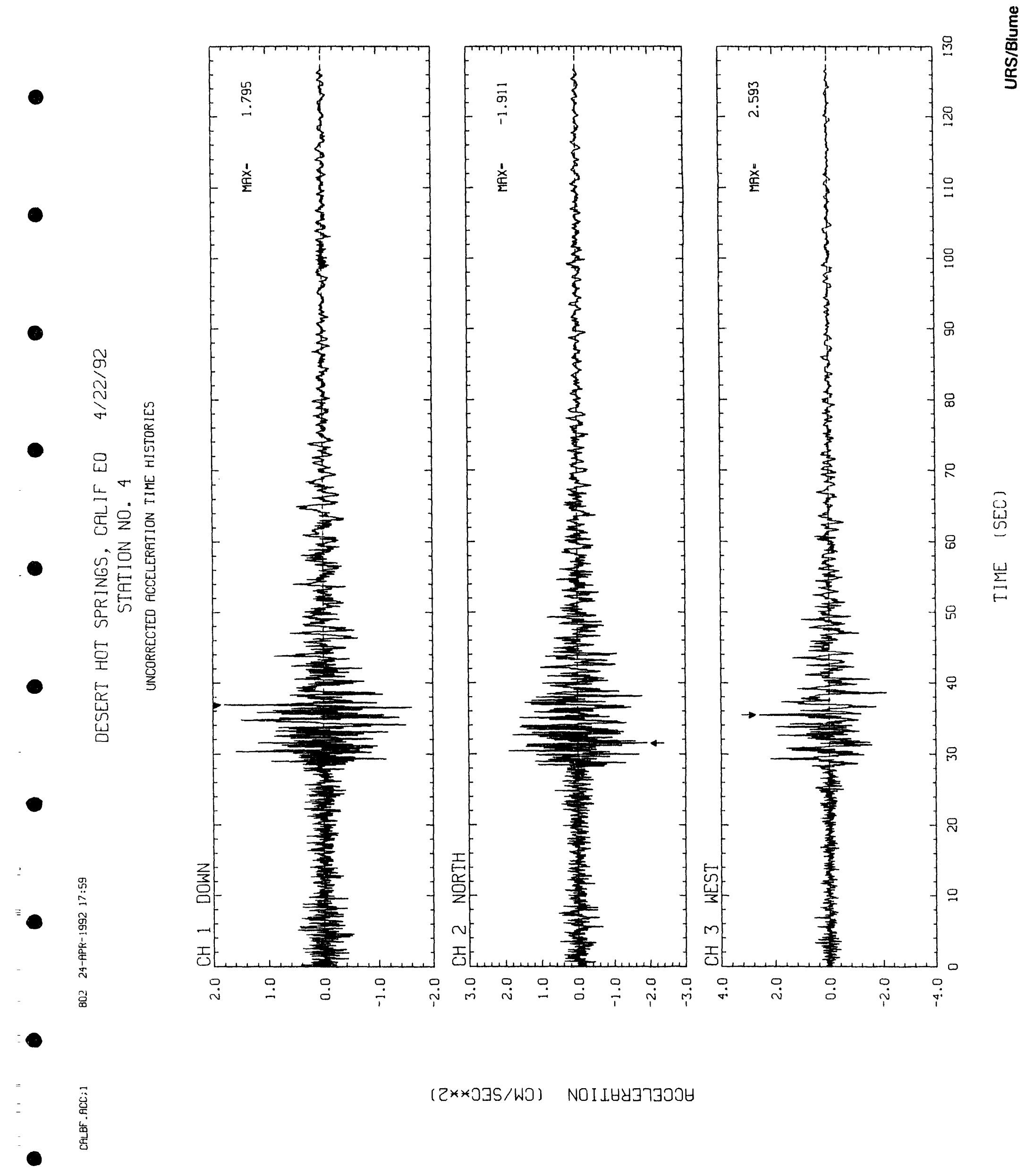



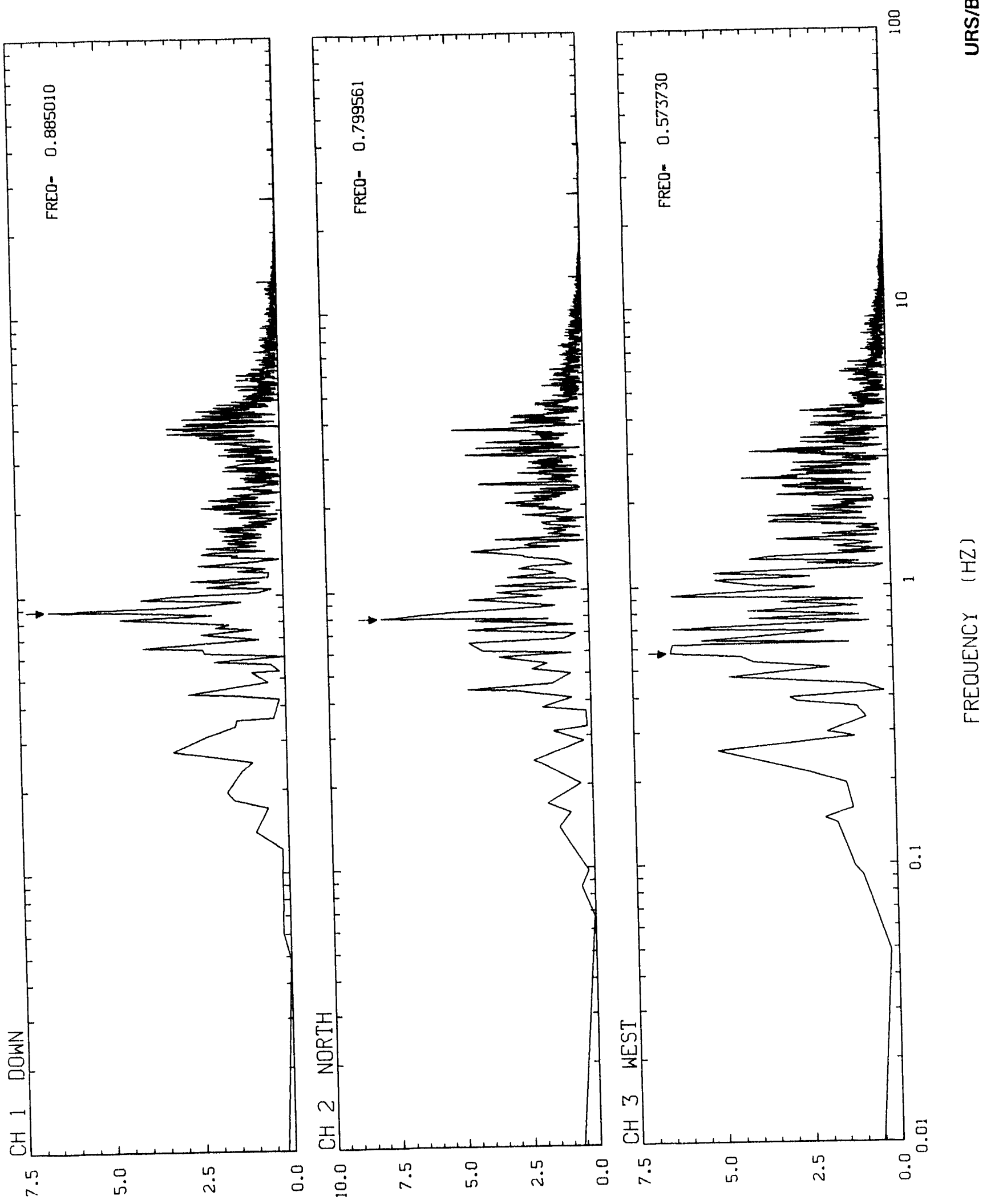


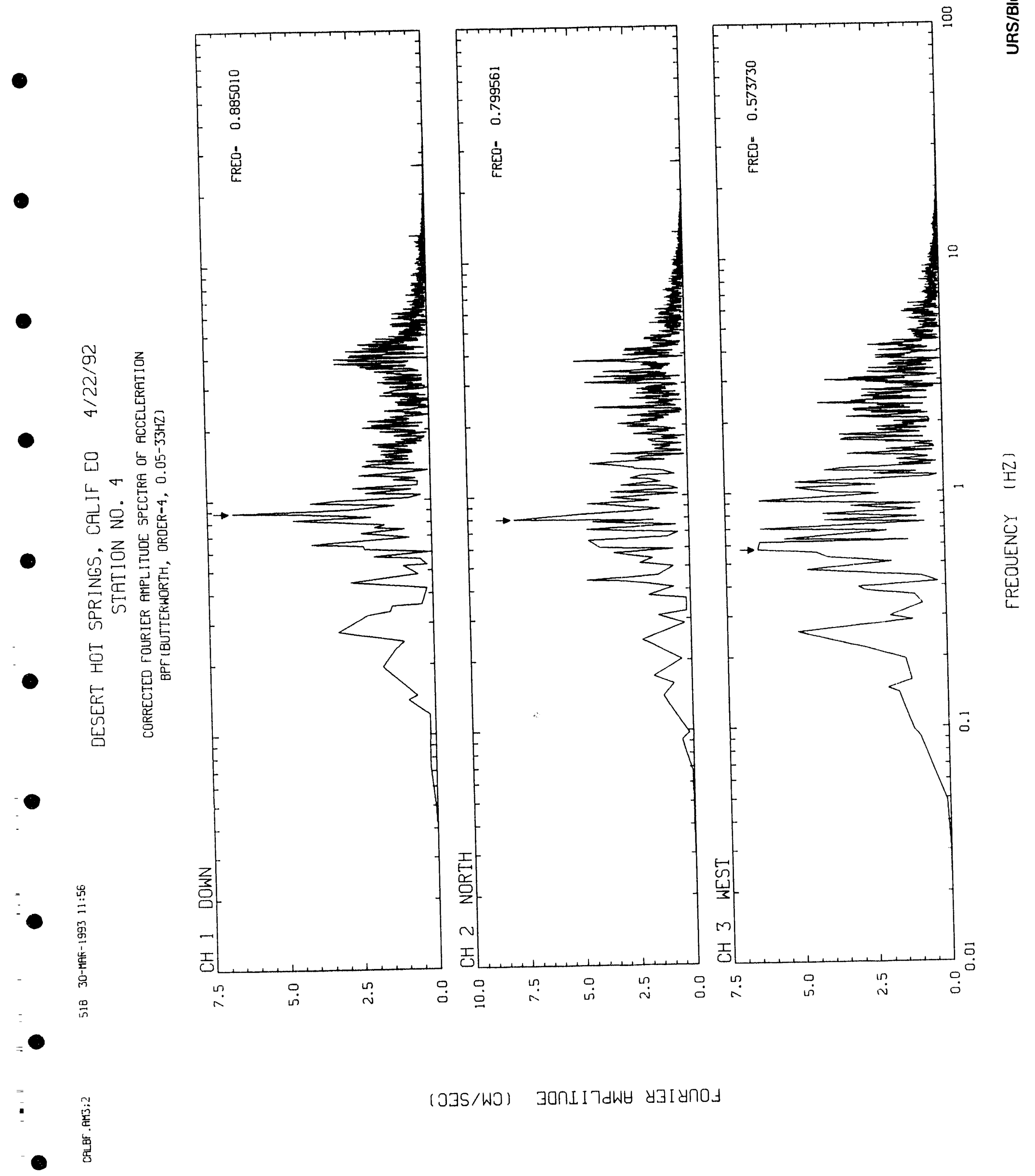


•

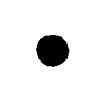

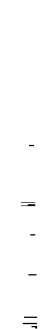
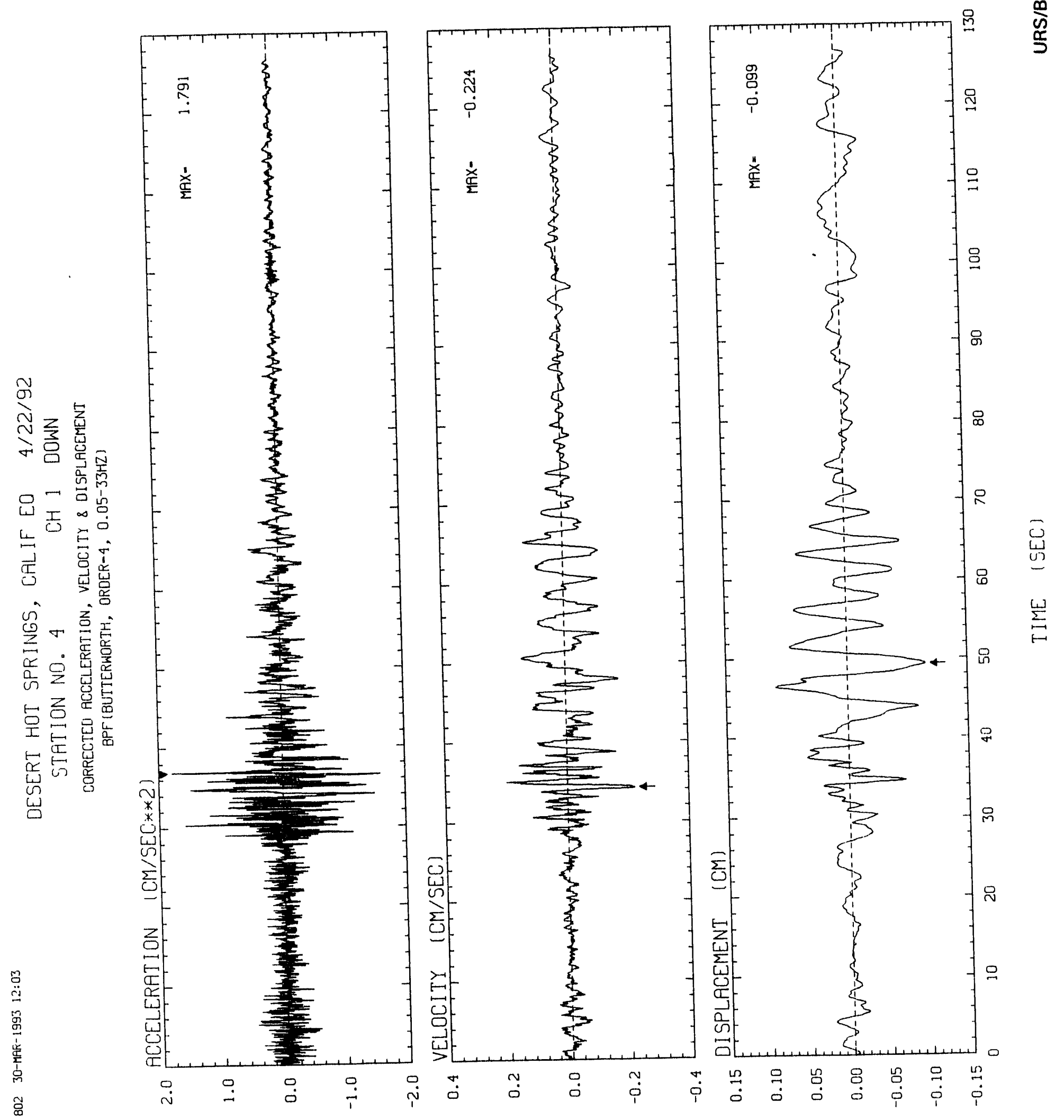


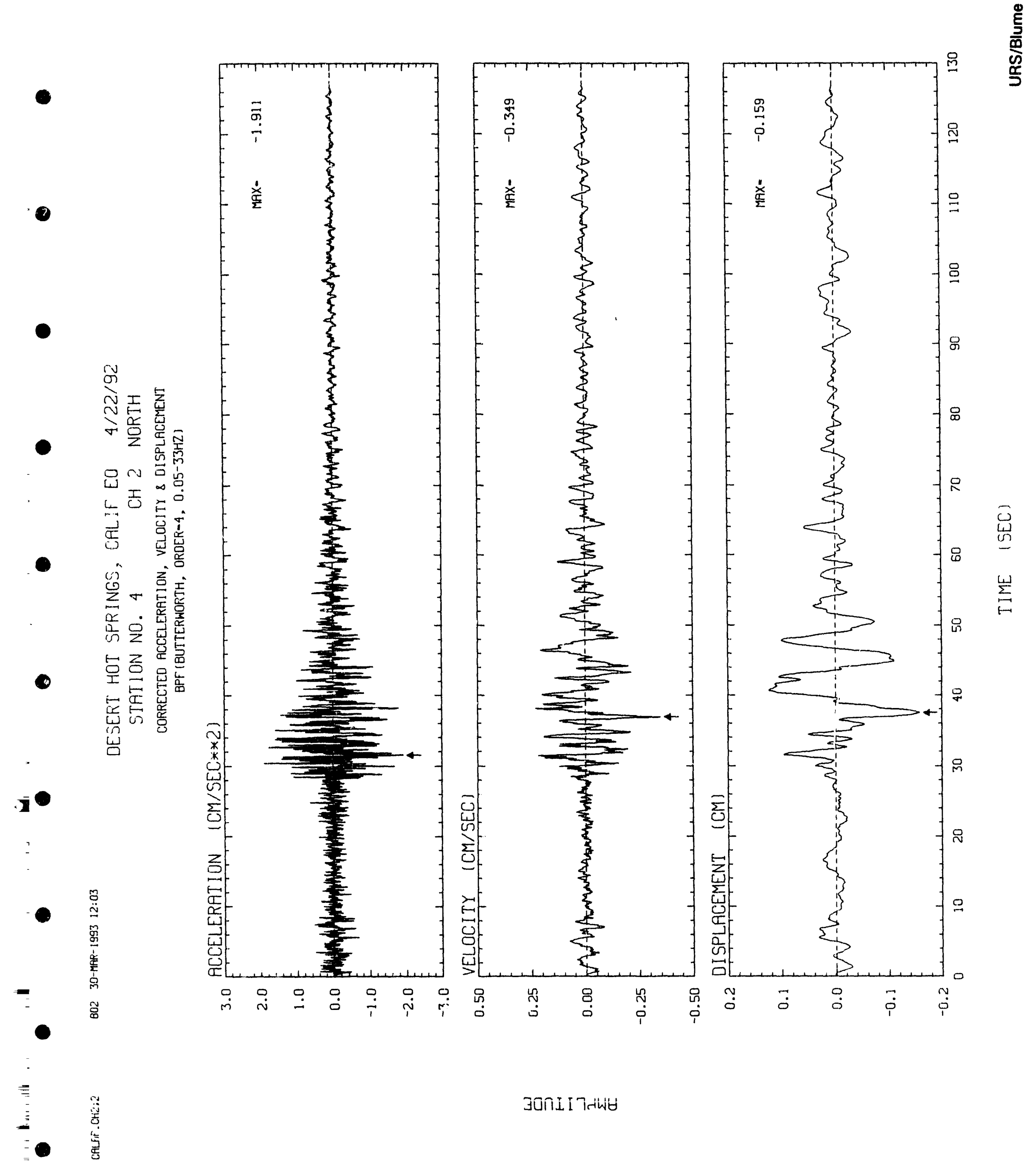




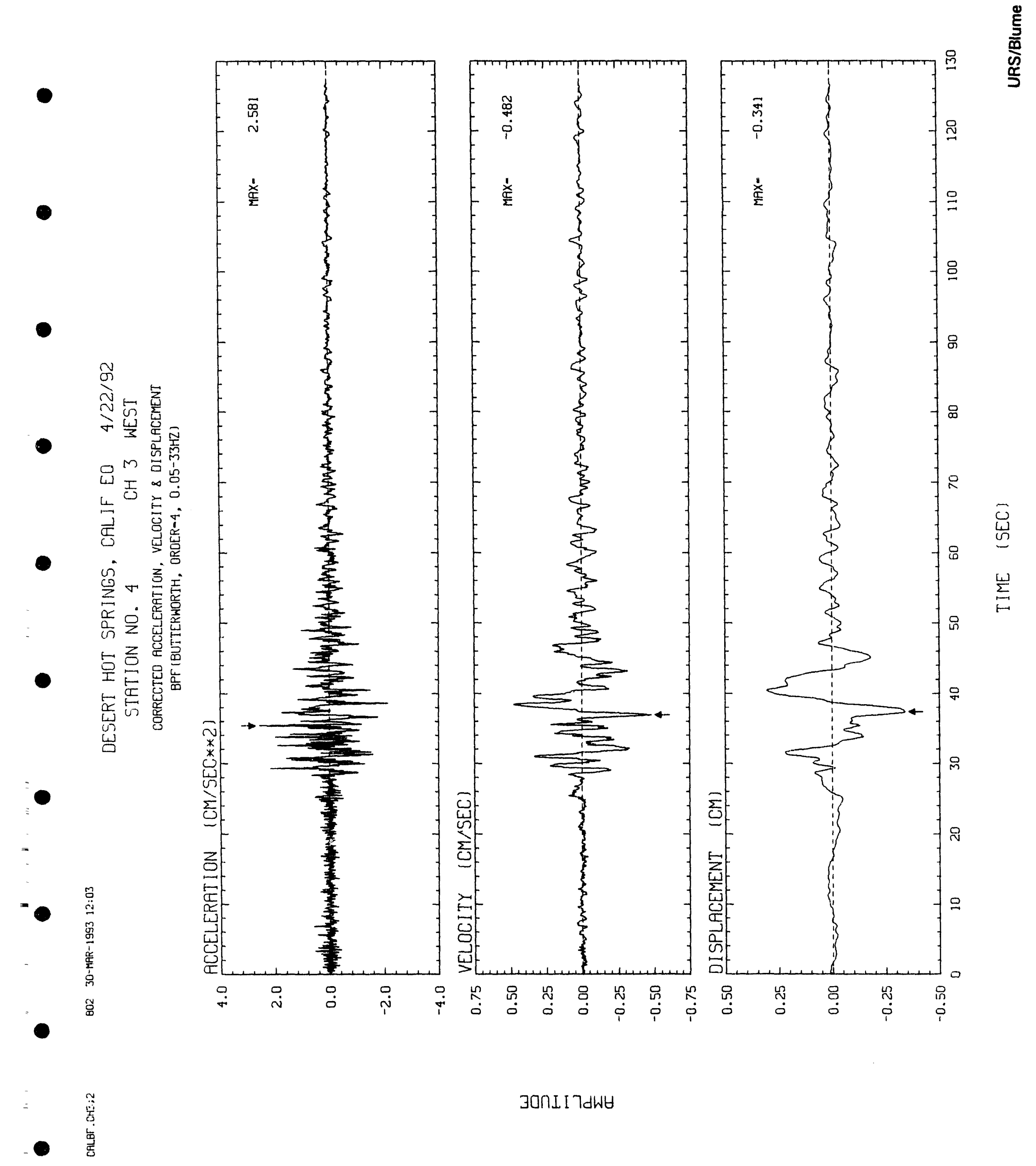



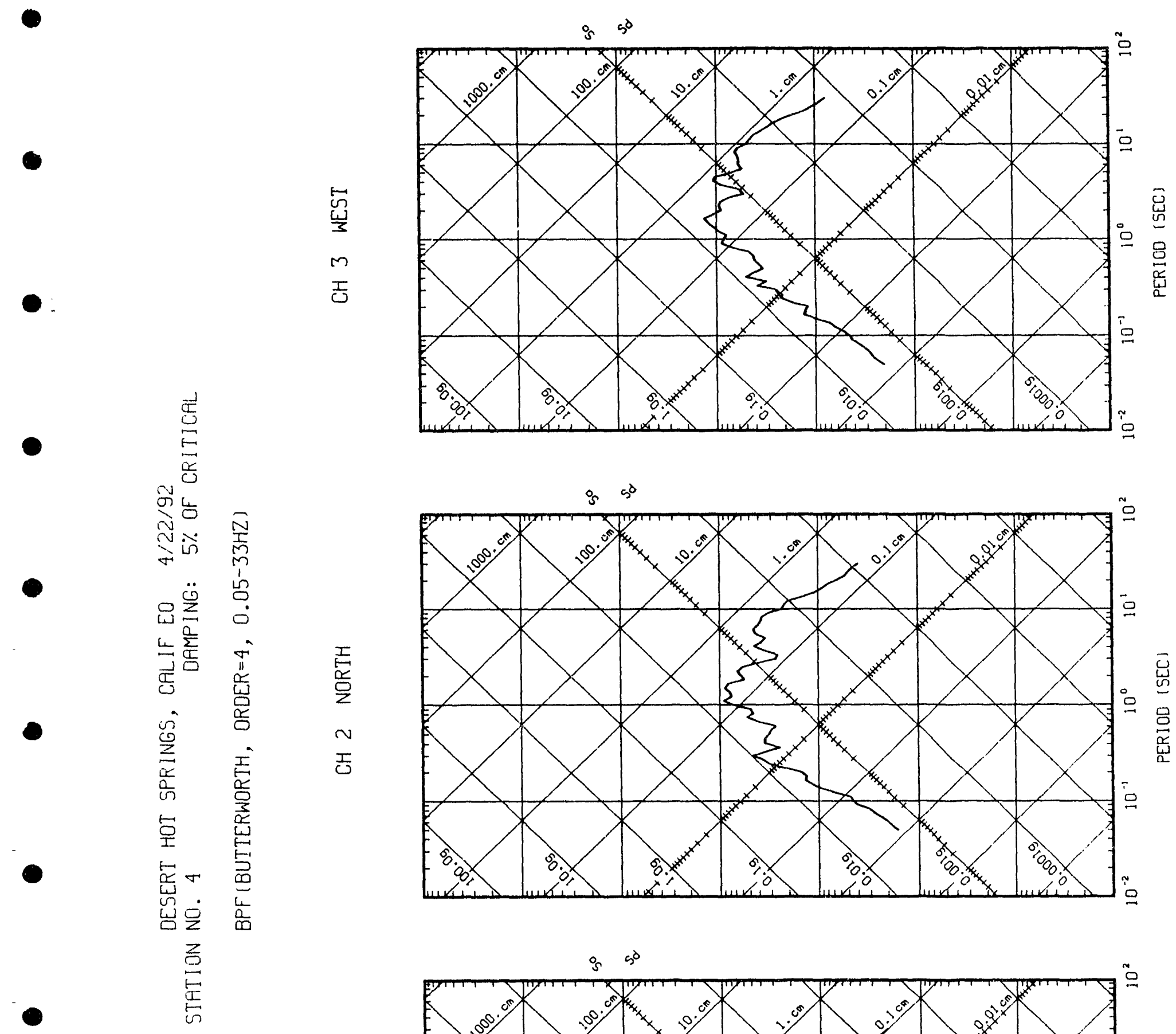

$\frac{\stackrel{9}{E}}{\stackrel{5}{0}}$
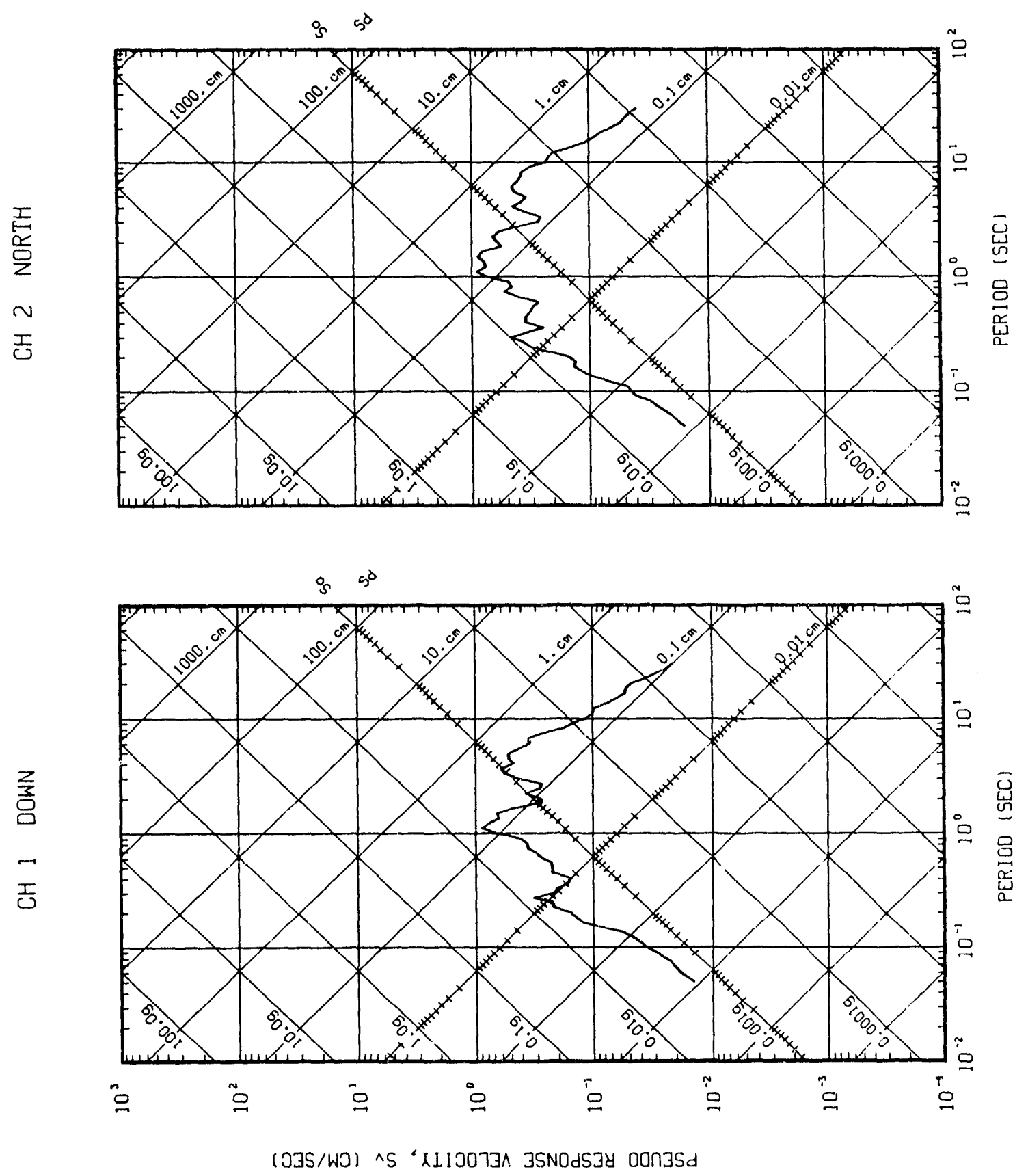


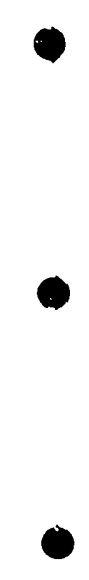



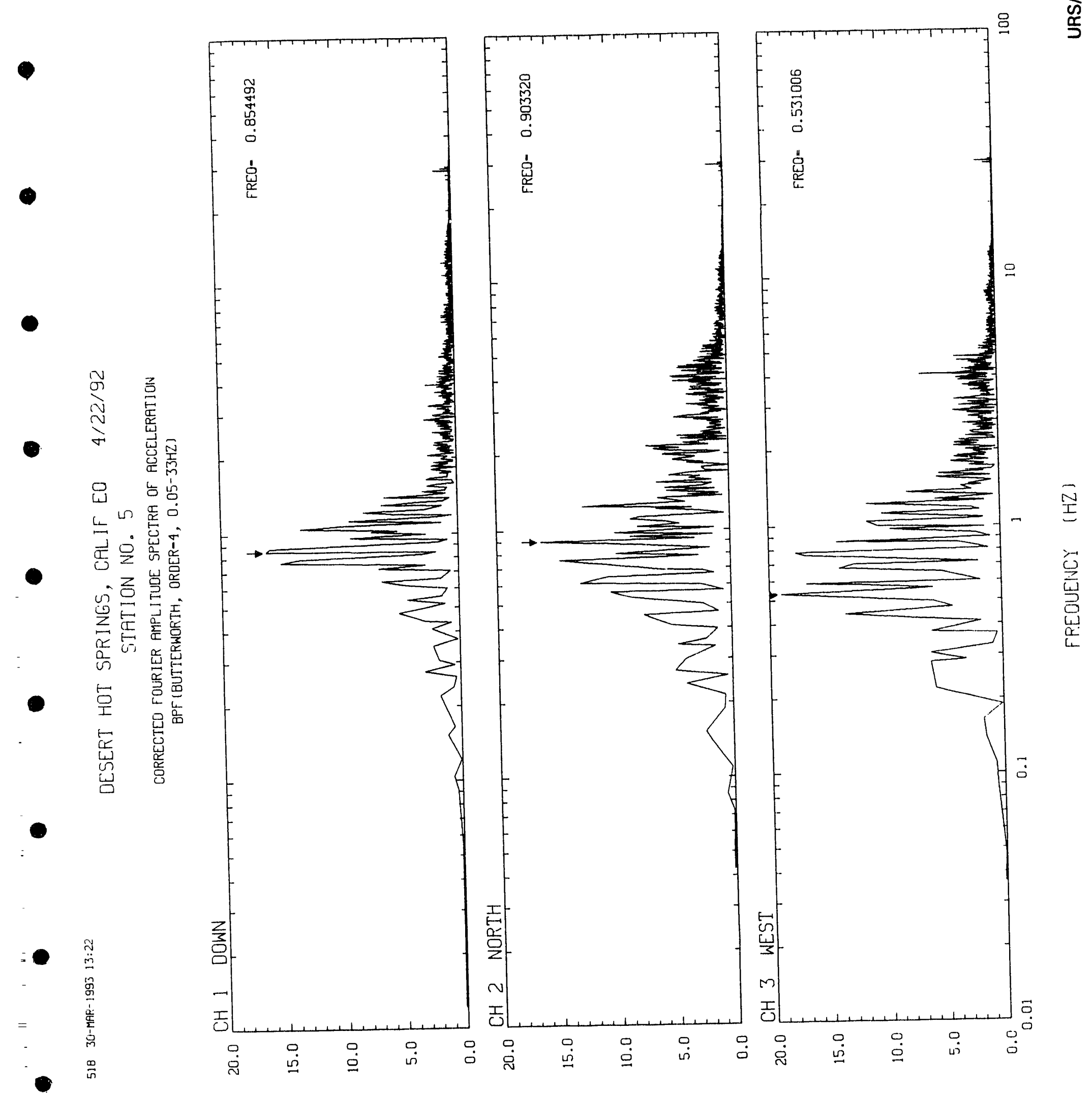


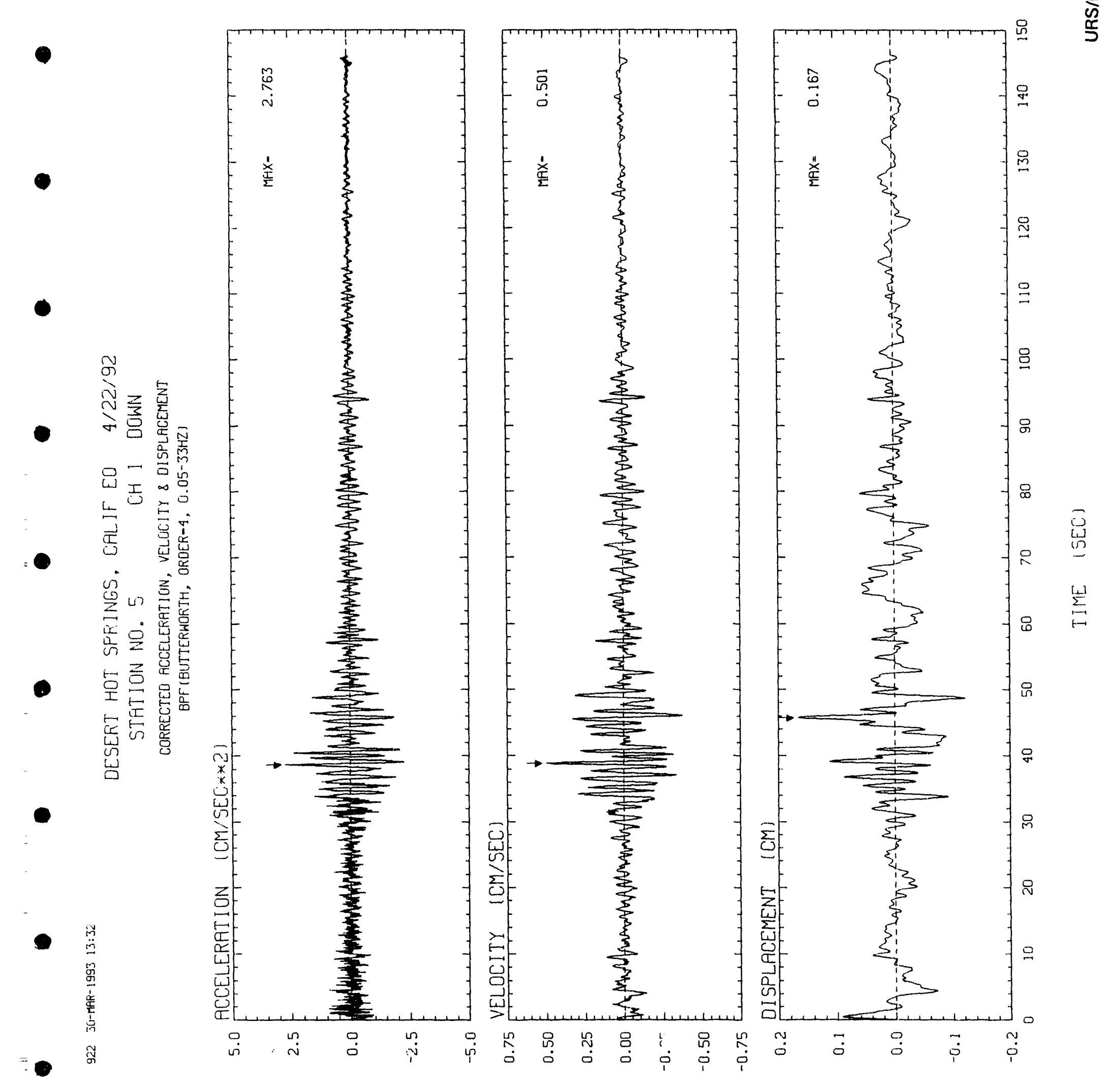


$\bullet$
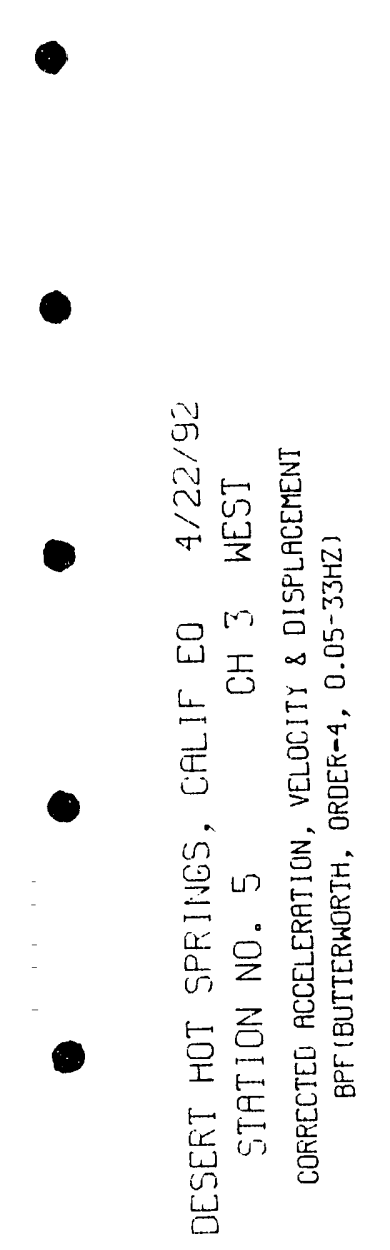

$\bullet$

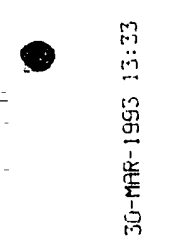

लु
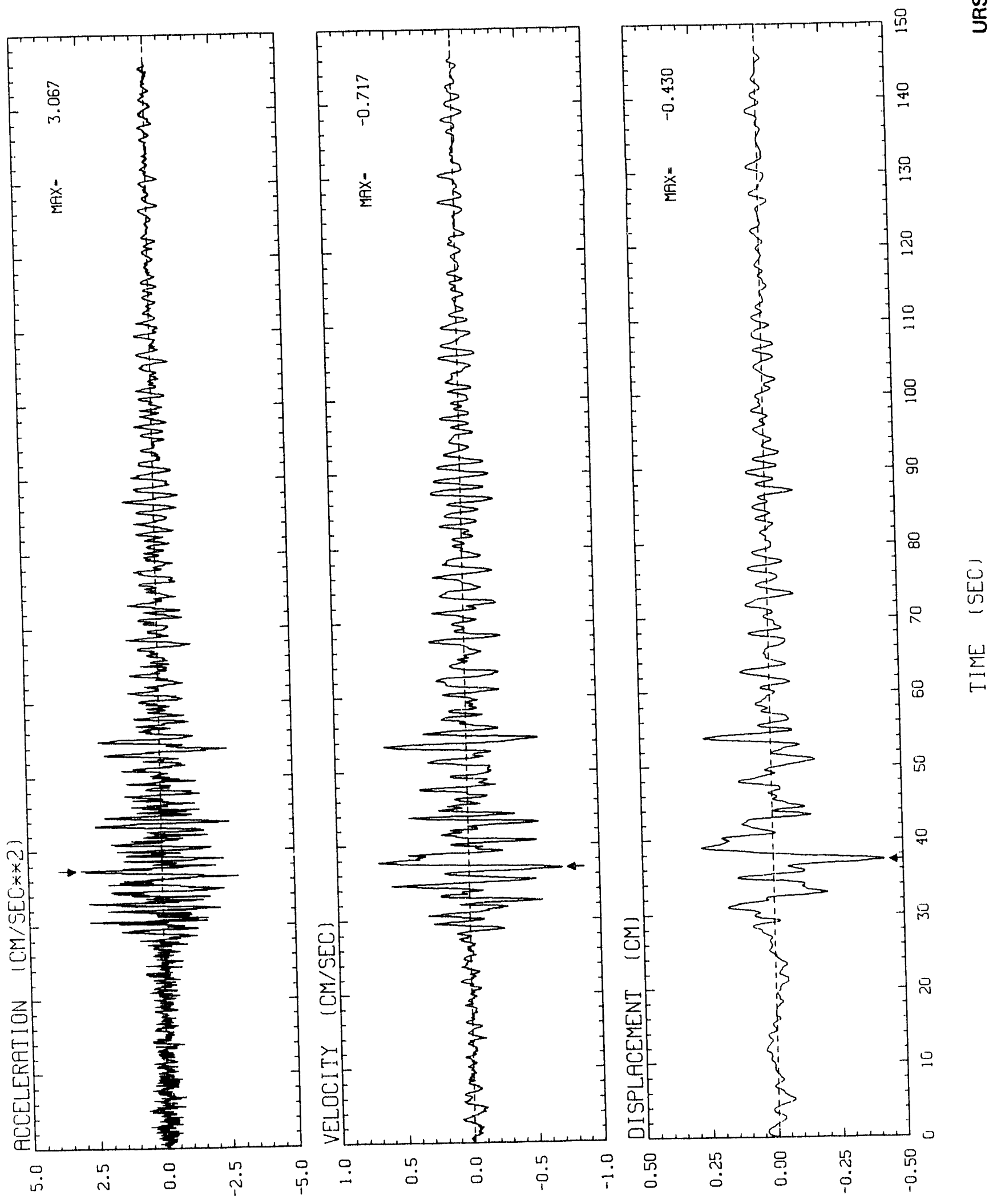

JOก I I 7dWH 


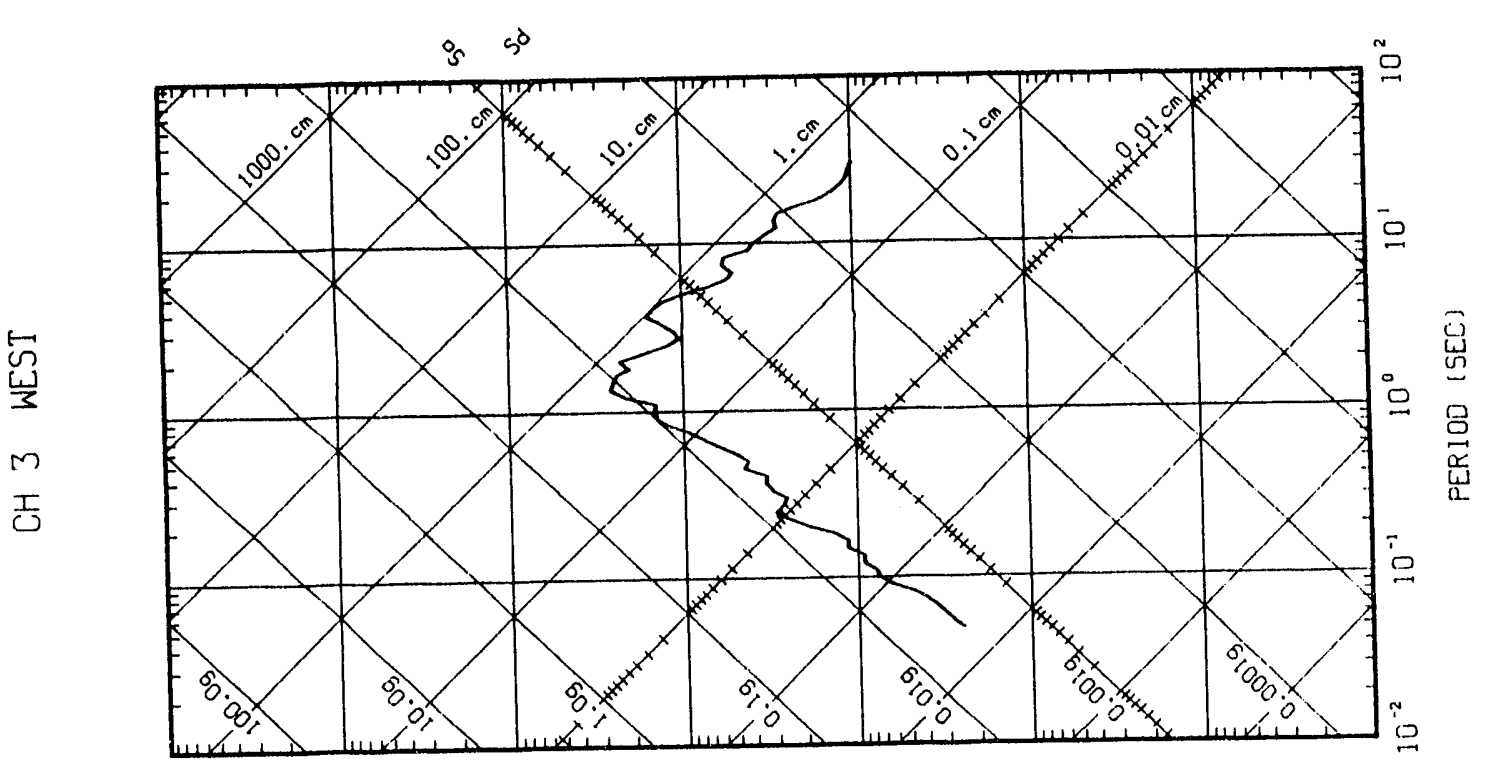

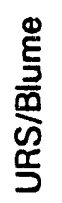
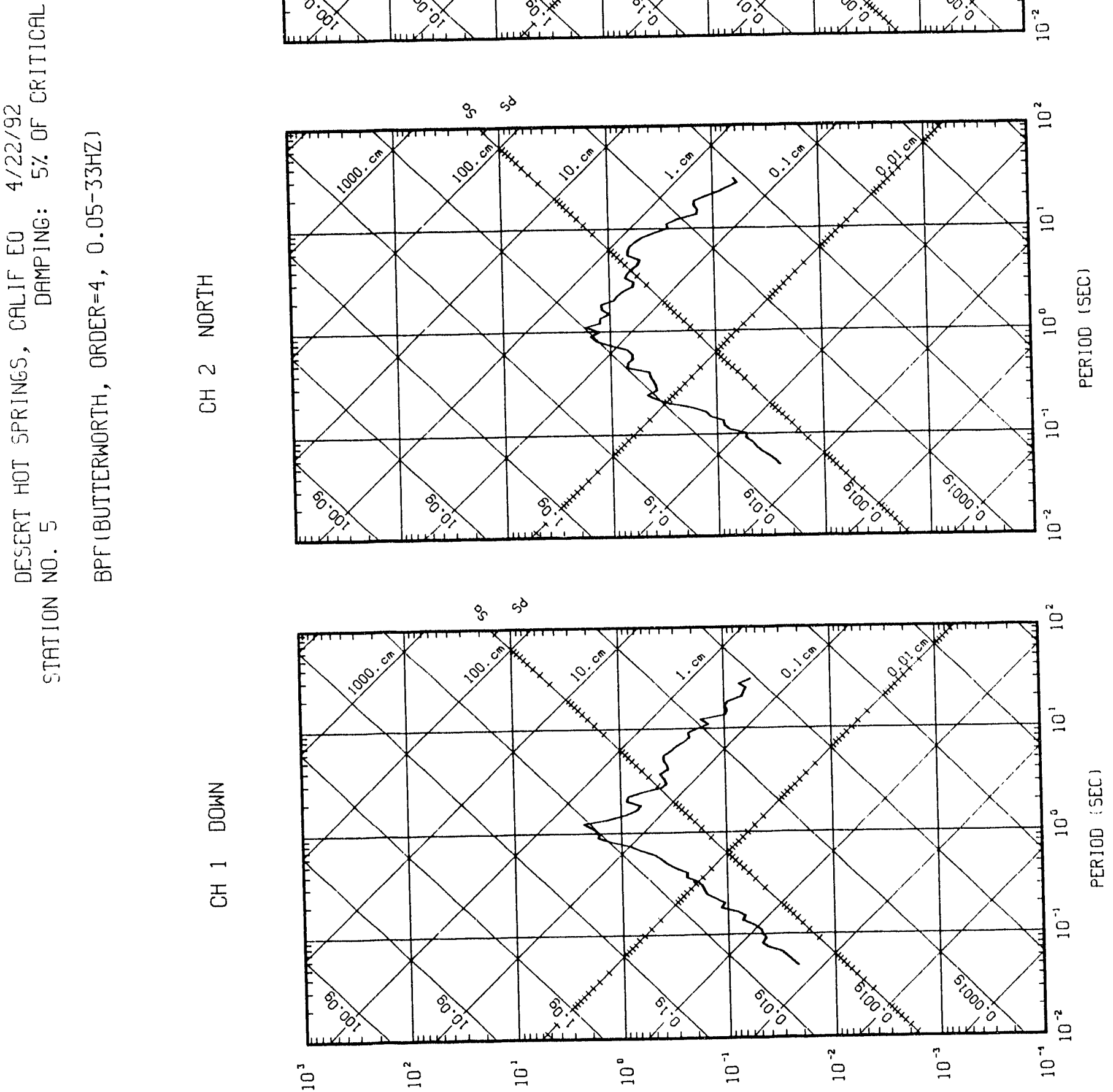
$\varangle$

0

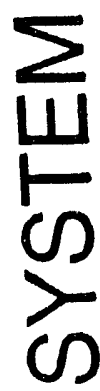

6

ํํㄹ

- 

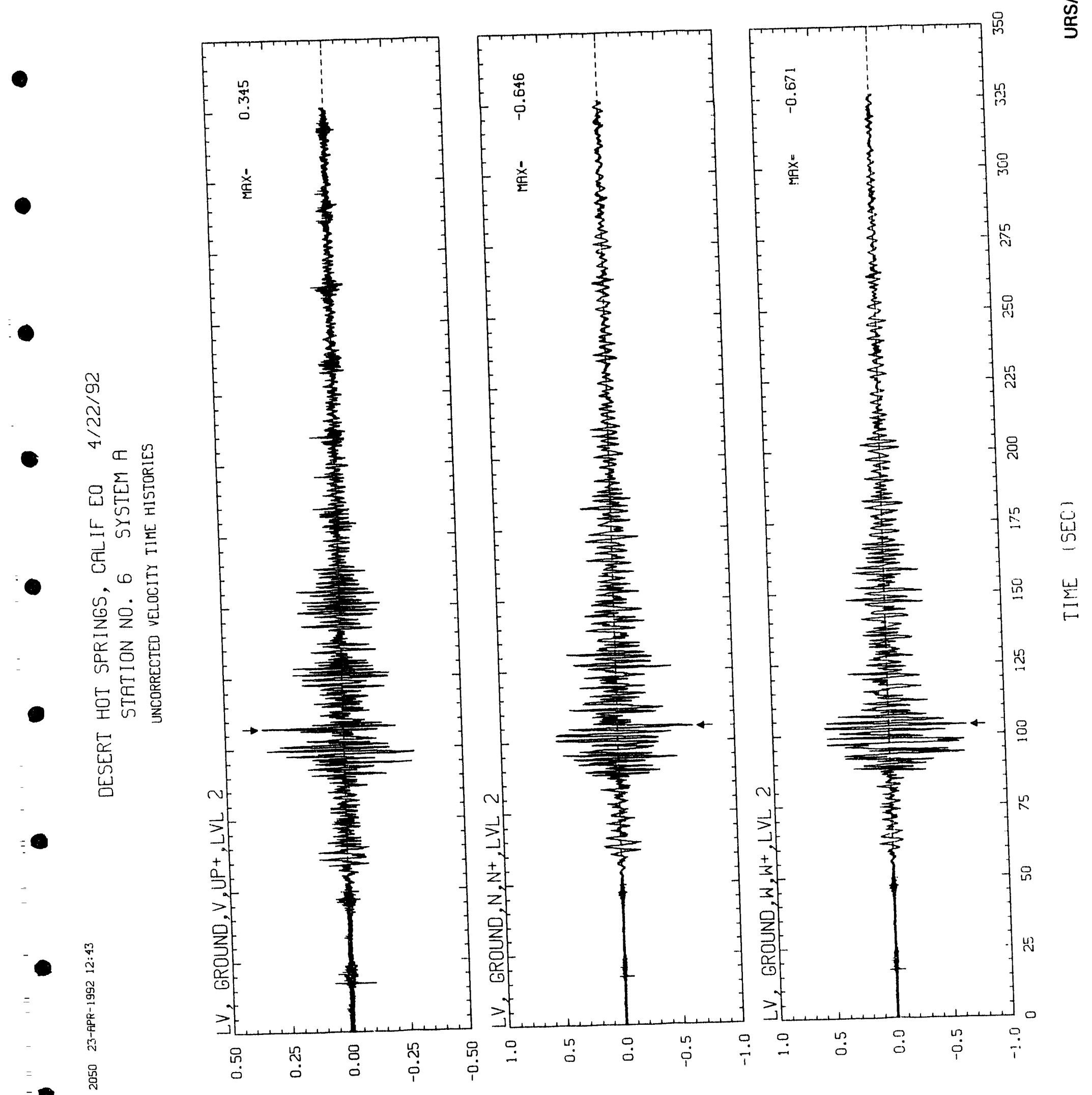

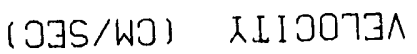




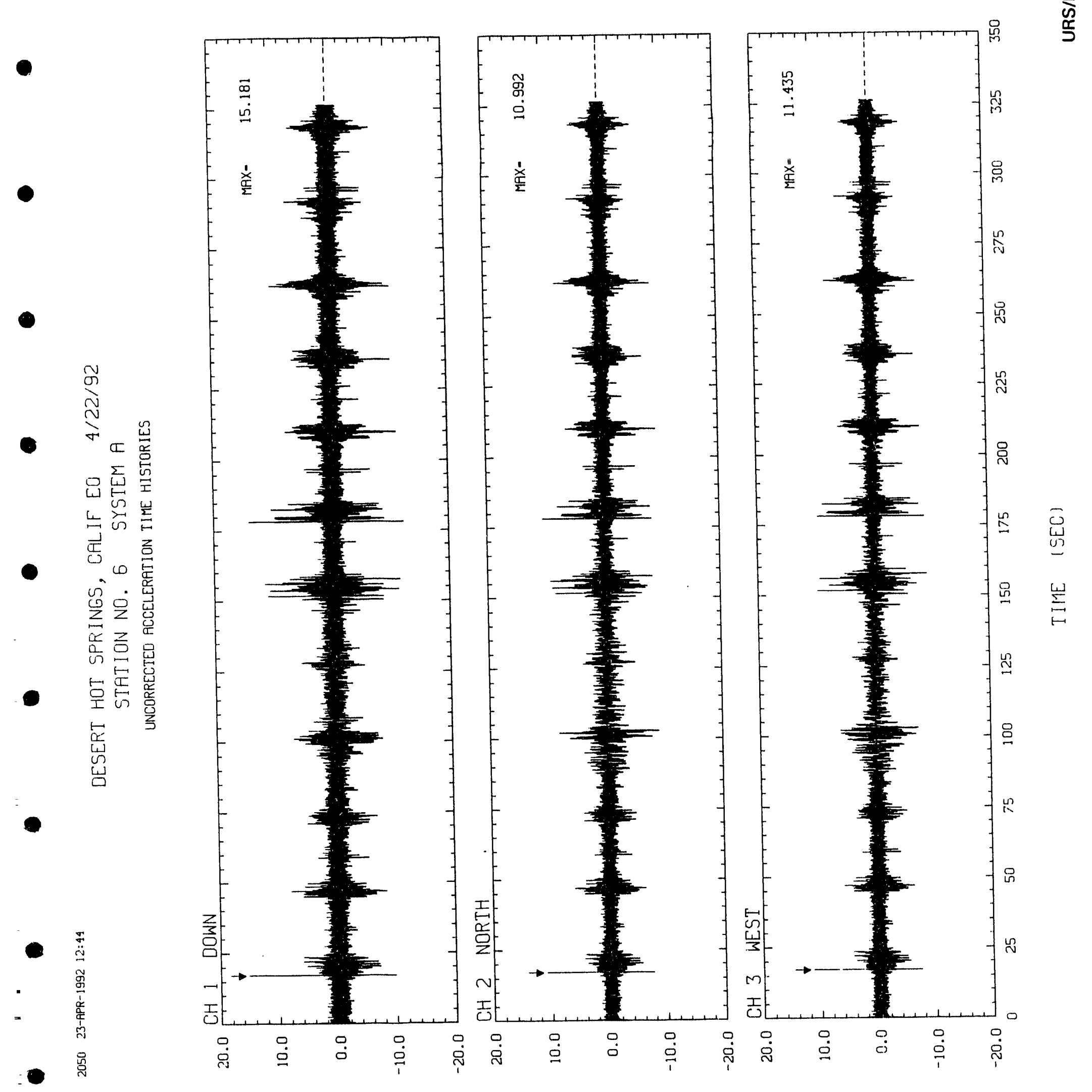



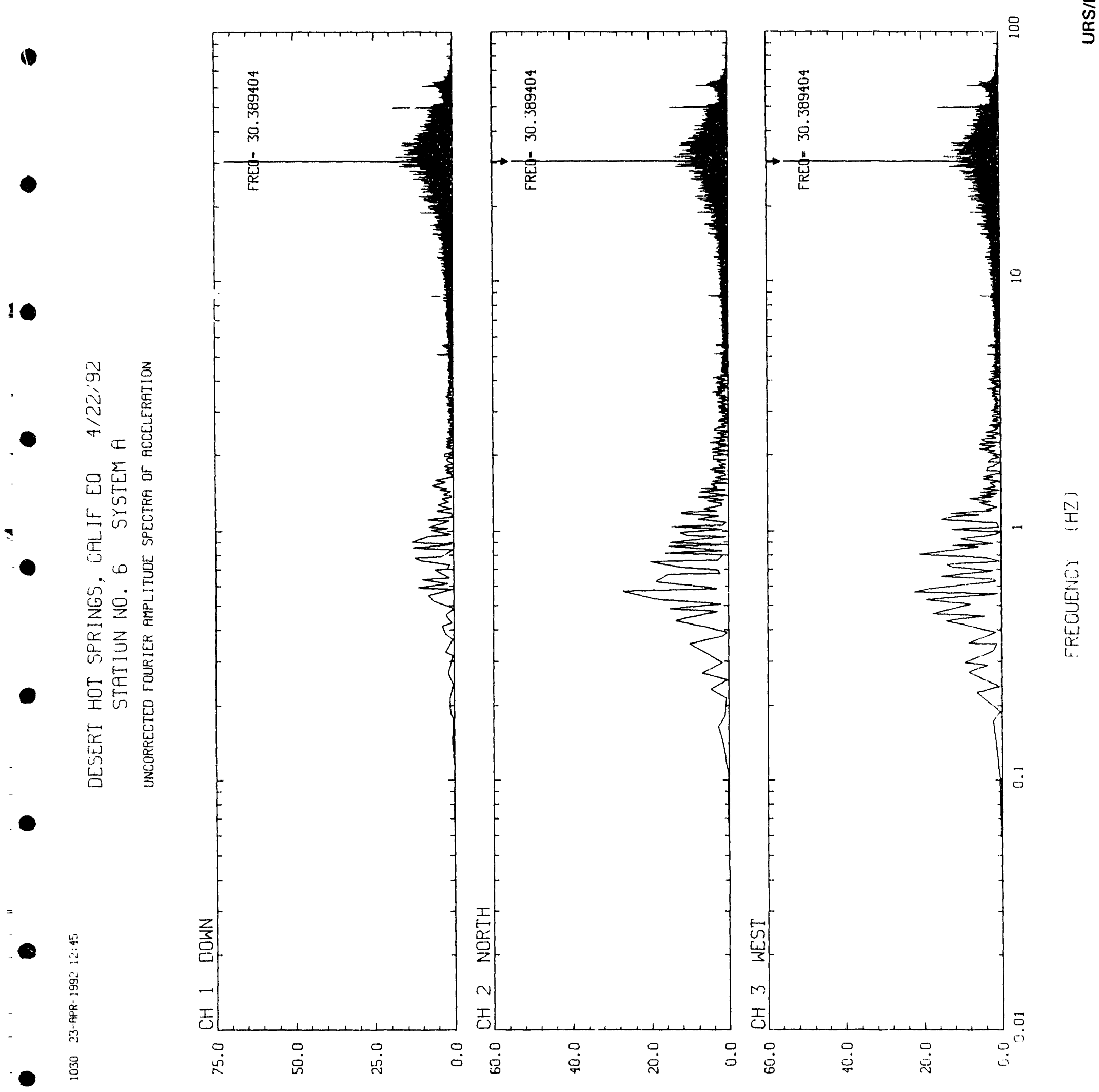

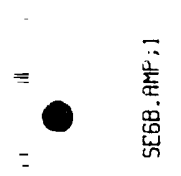



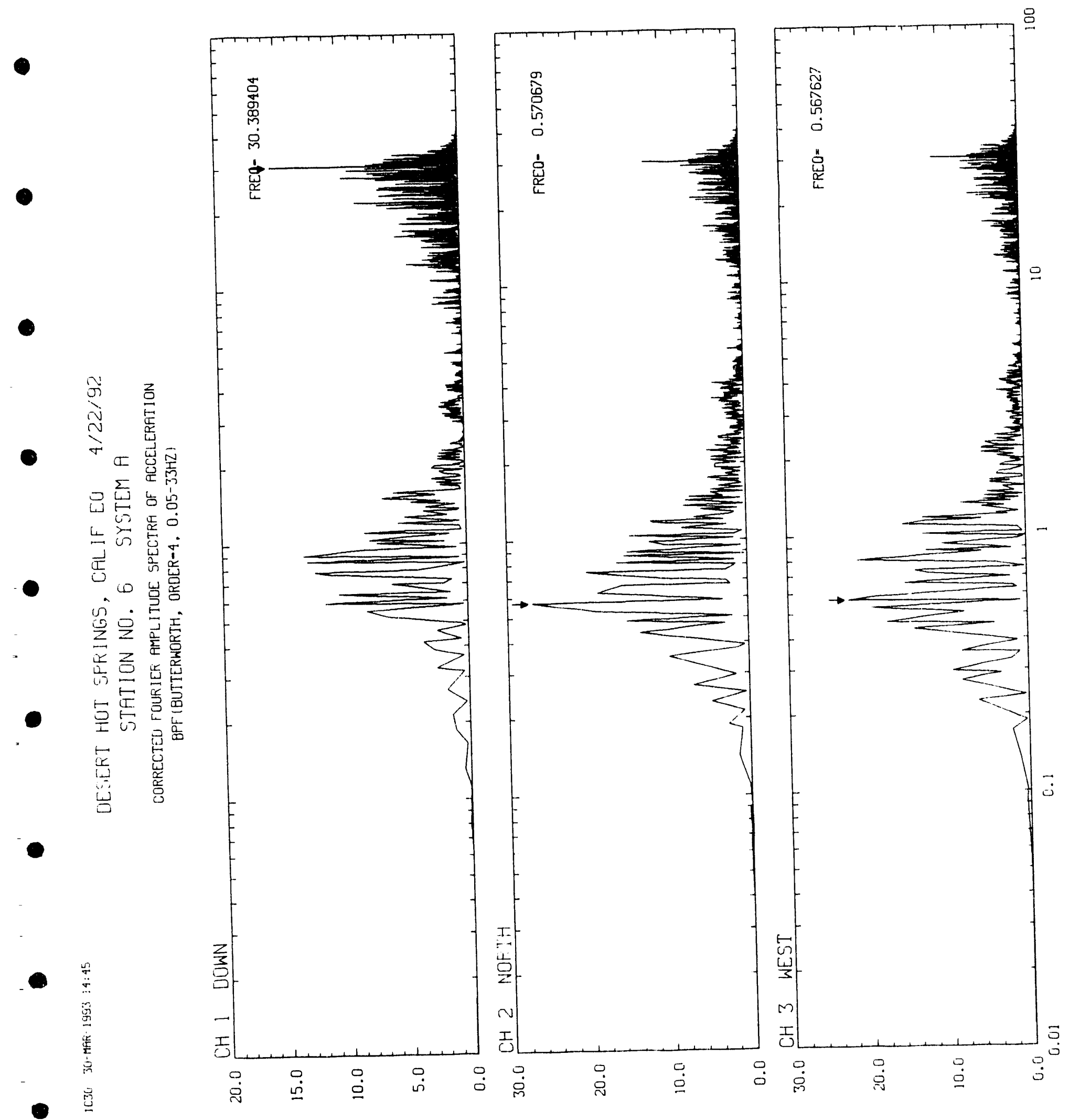

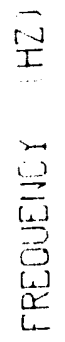



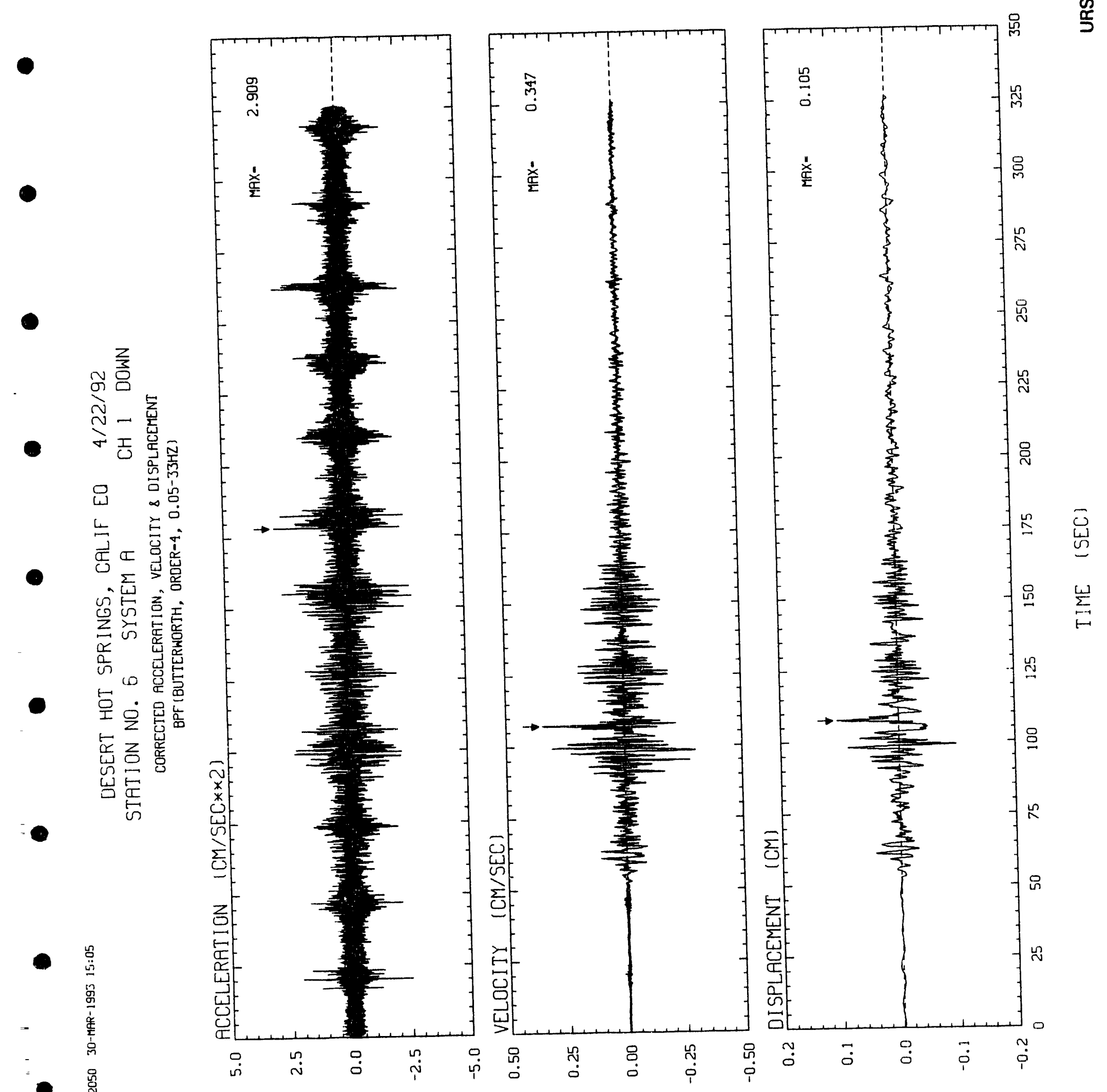

- 


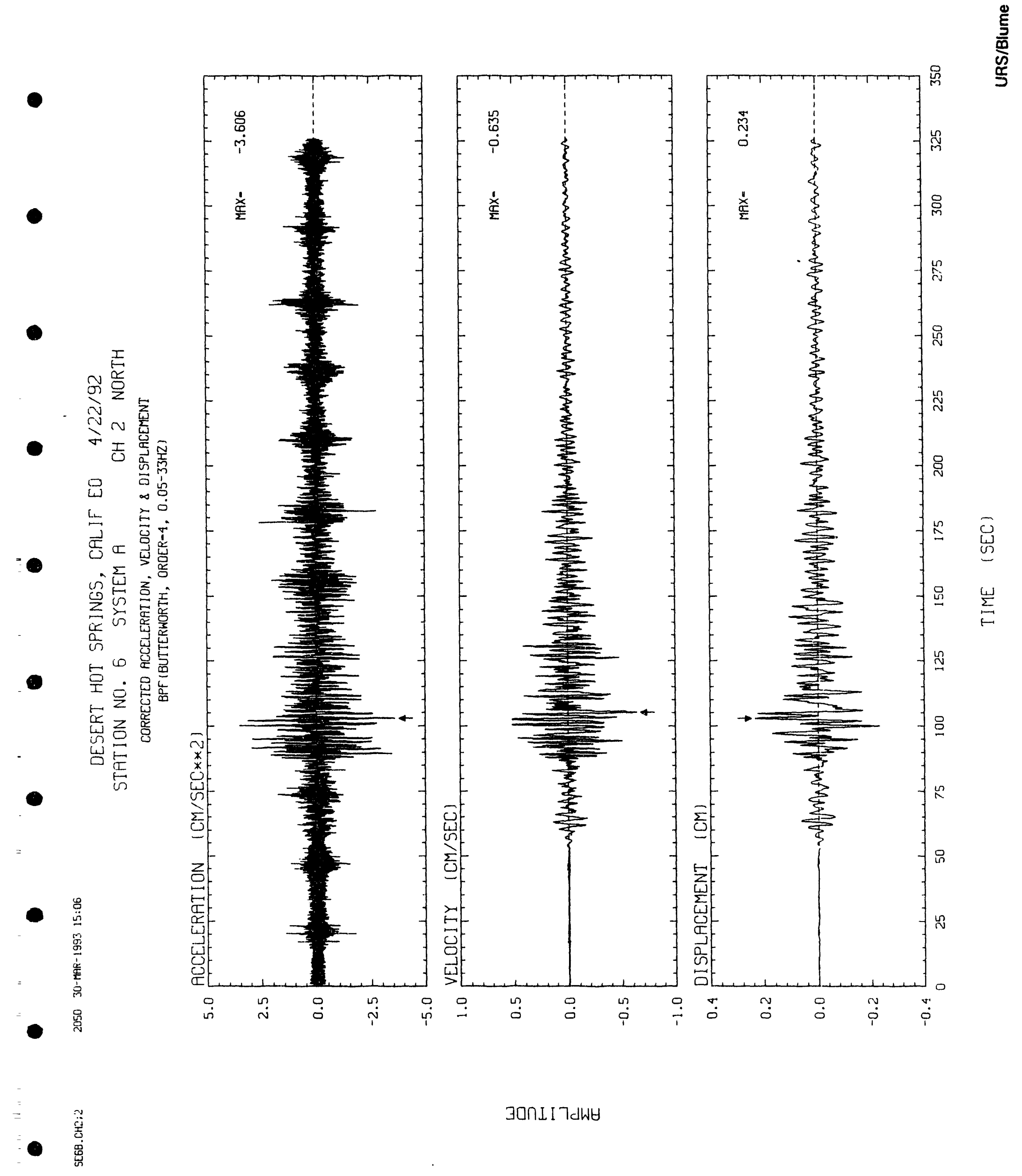



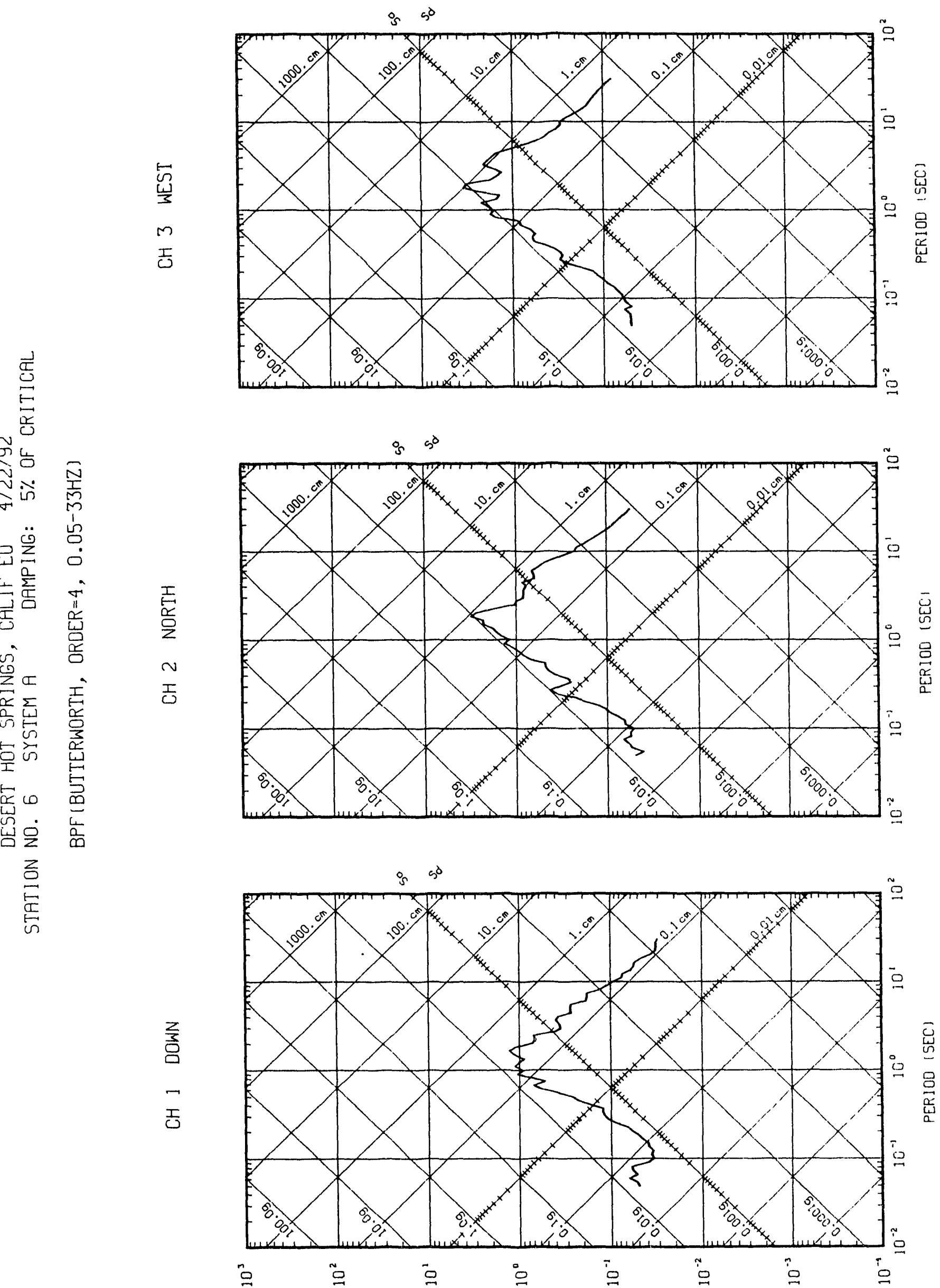

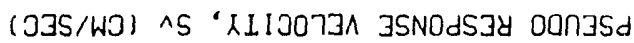



$\stackrel{5}{\omega}$

○ 

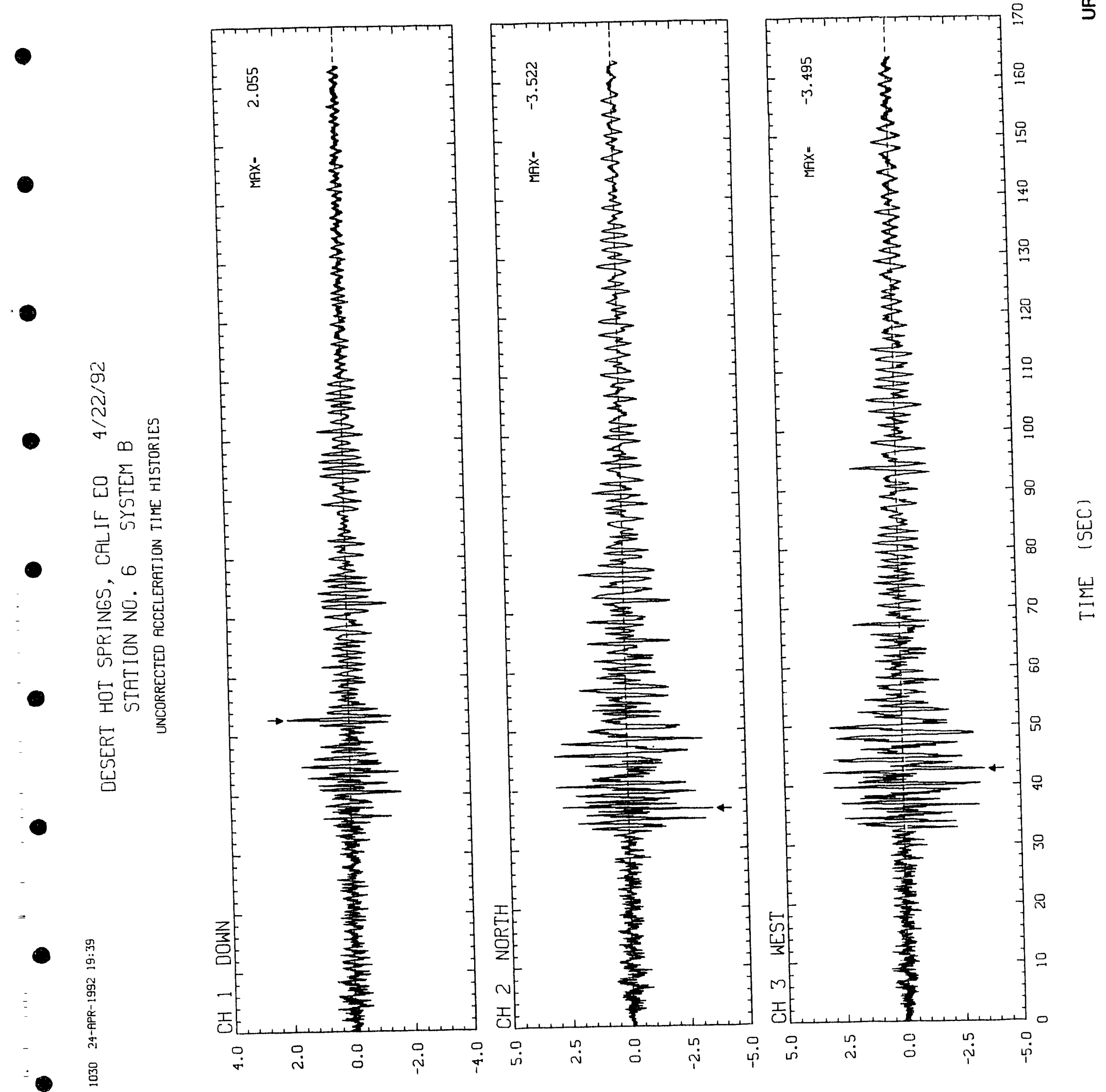

(2**035/WJ) NOI I $4 y 27.7303$ 


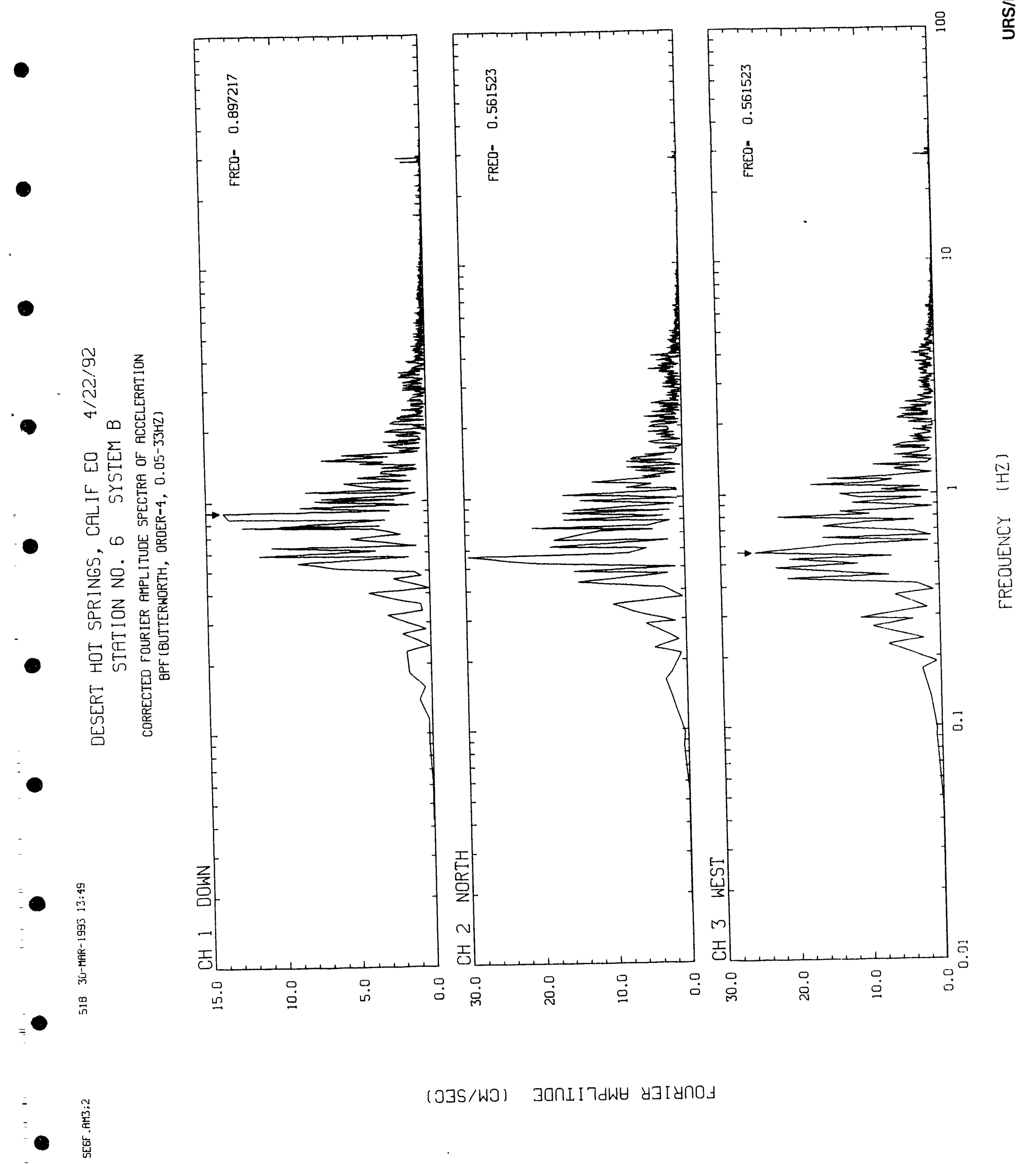



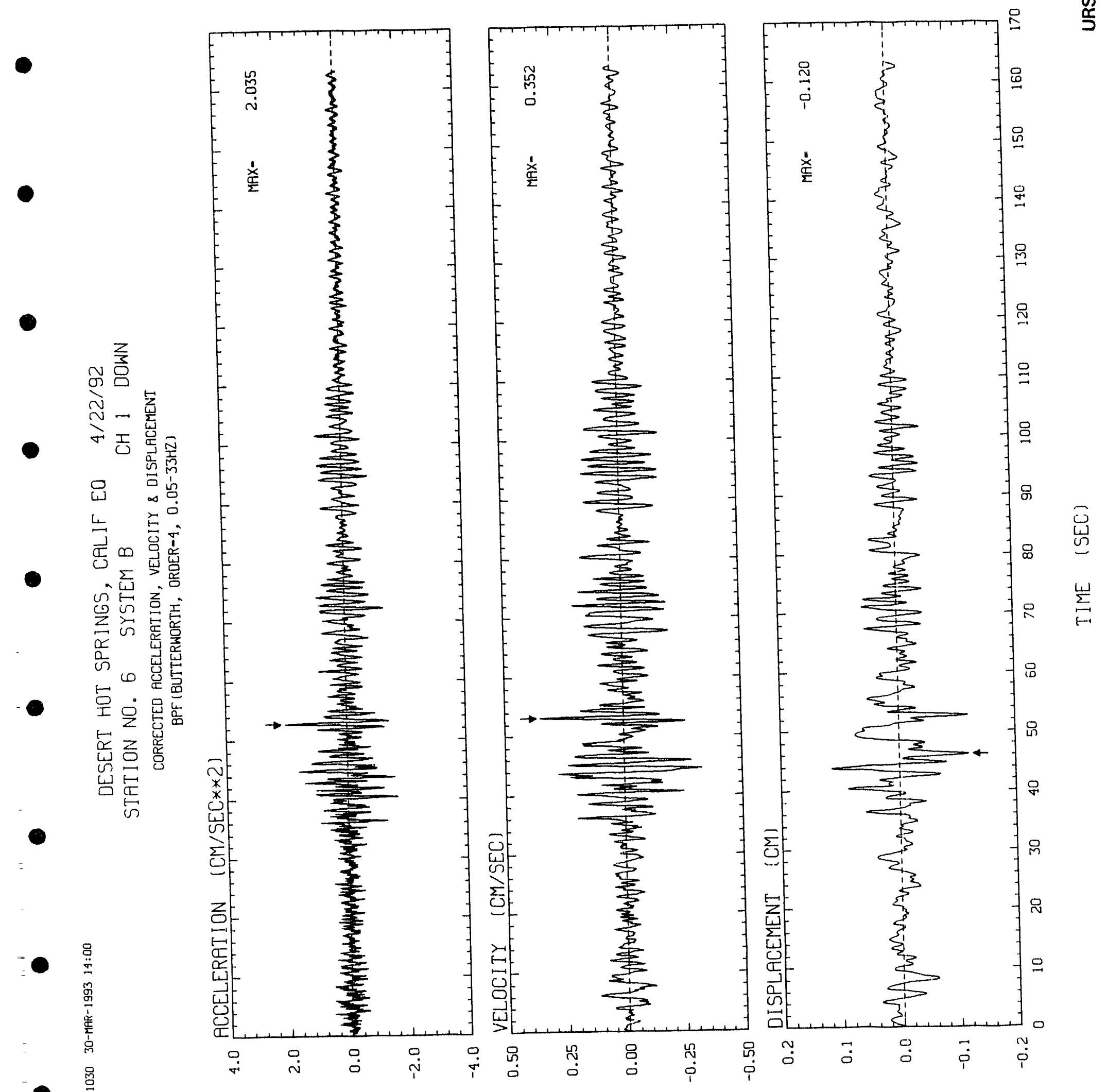


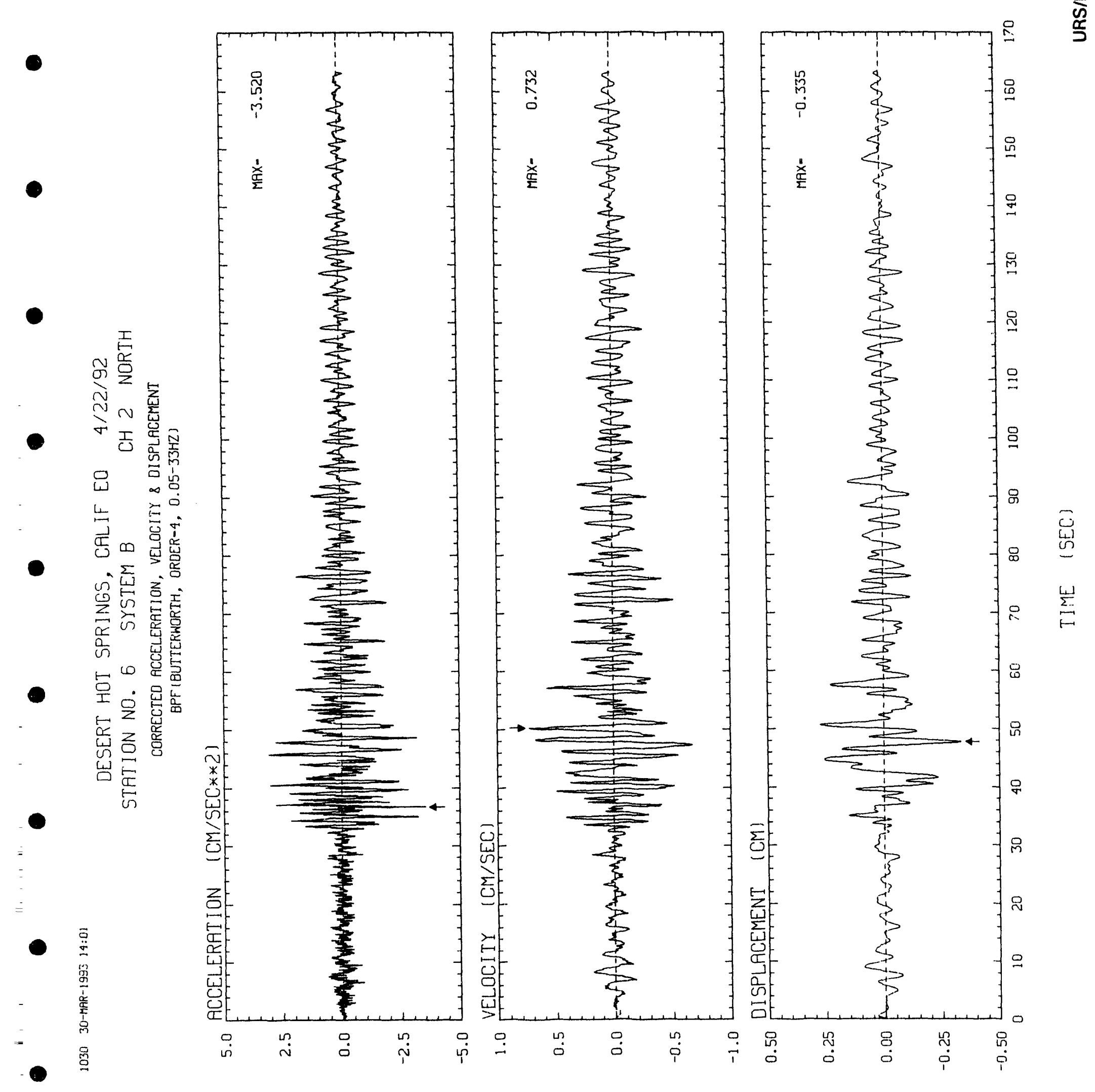



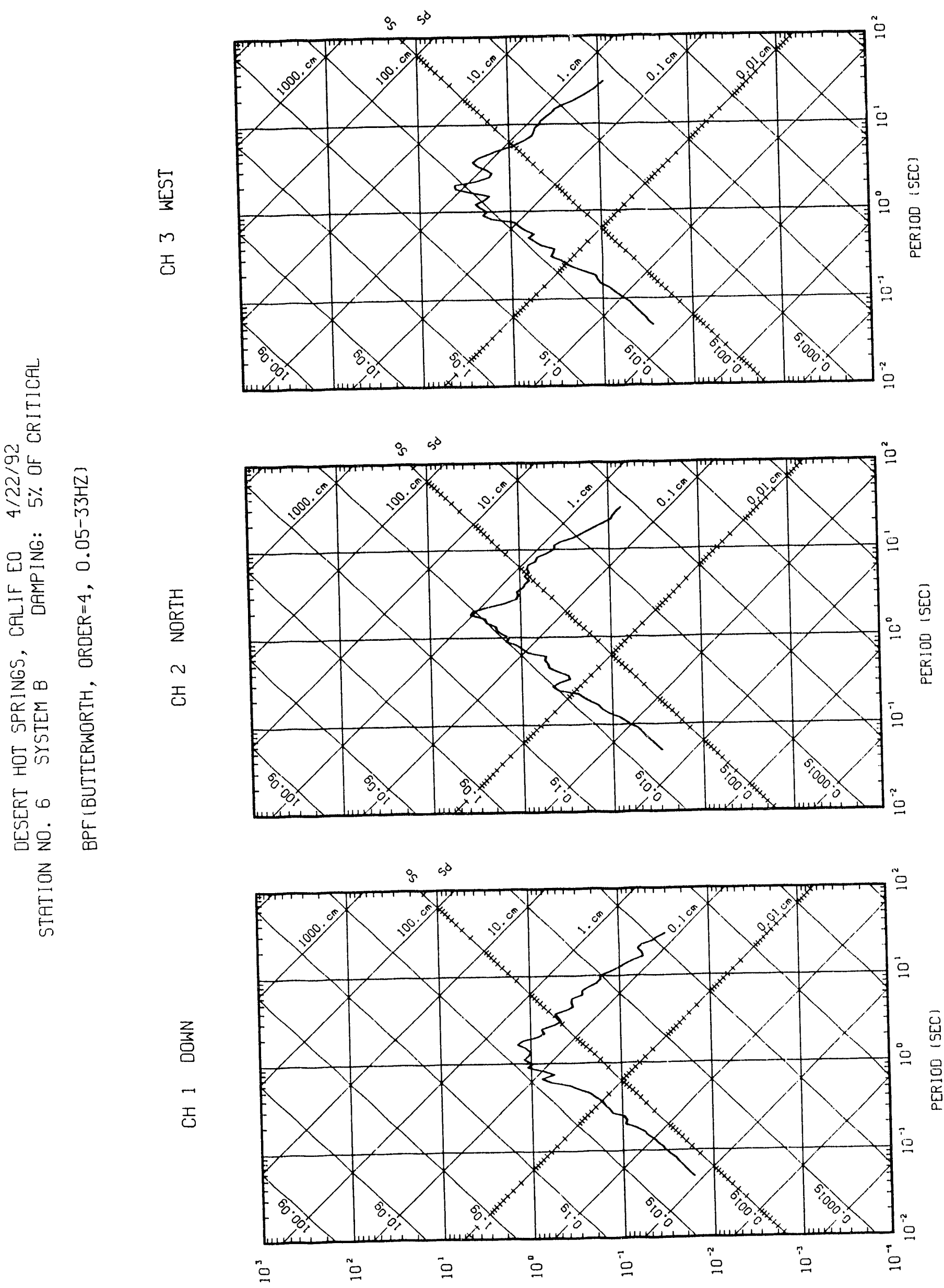

(J3S/WJ) ^S 'KLIJOTJM JSNOdSJY OONJSd 


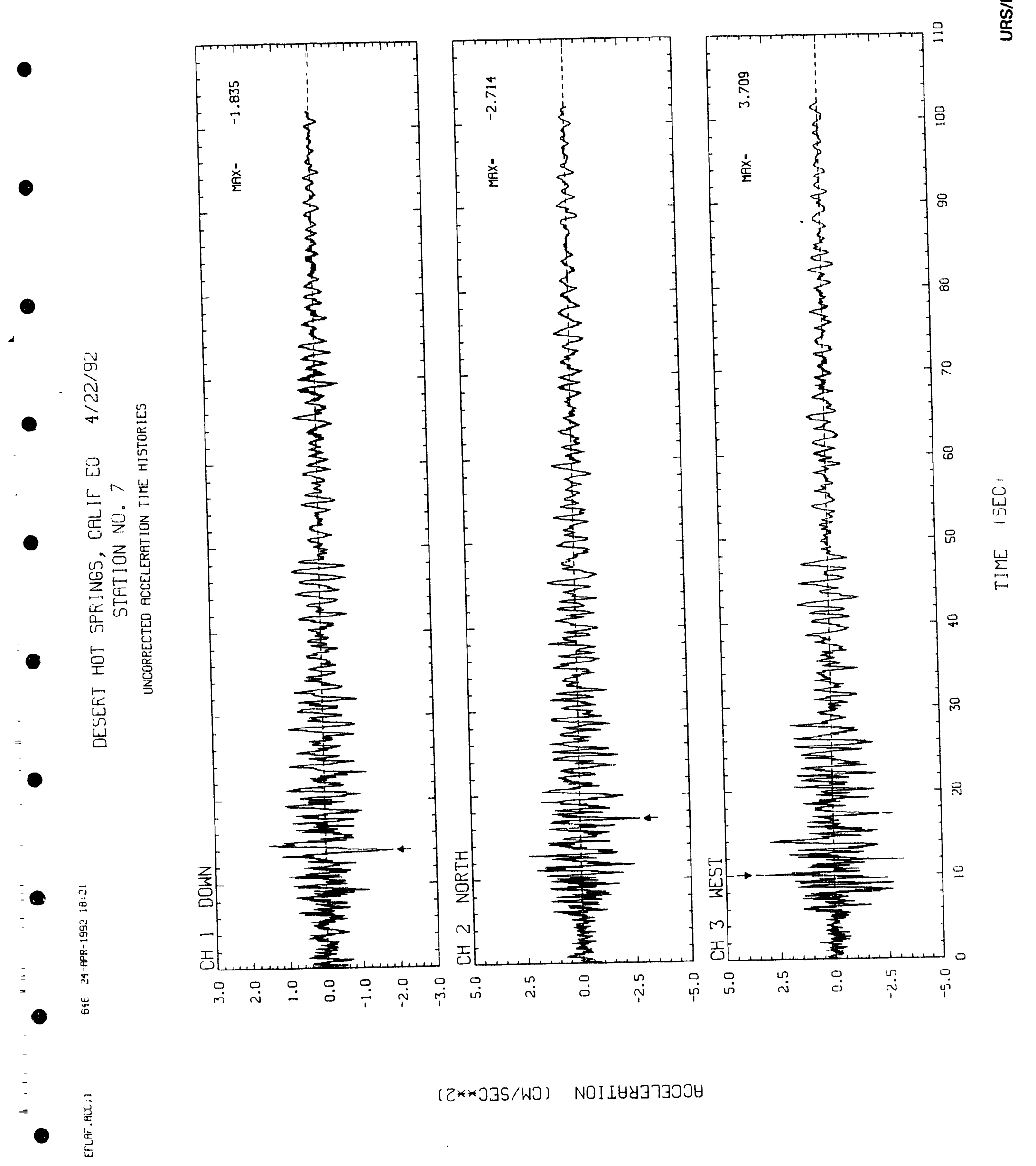




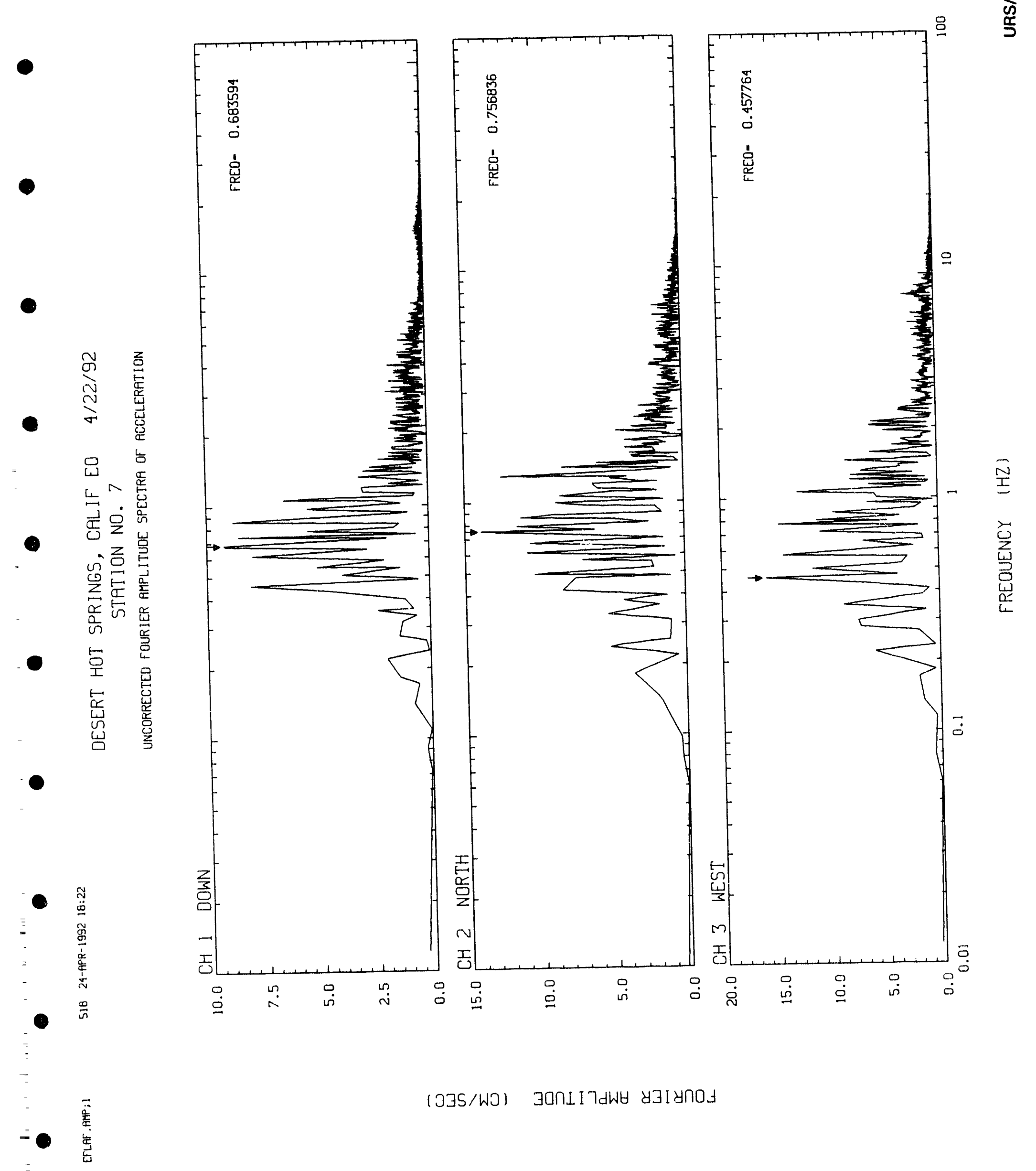



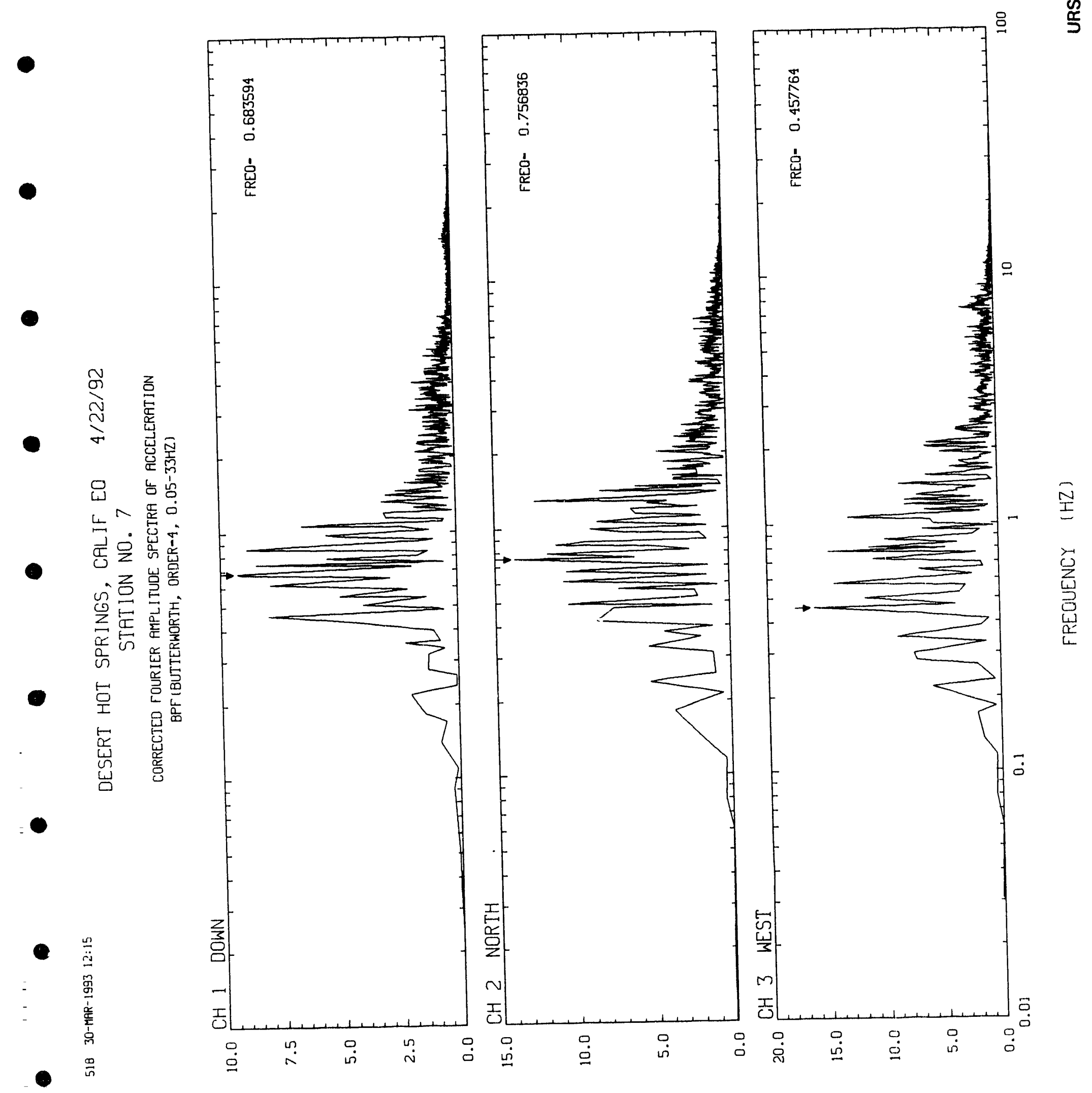


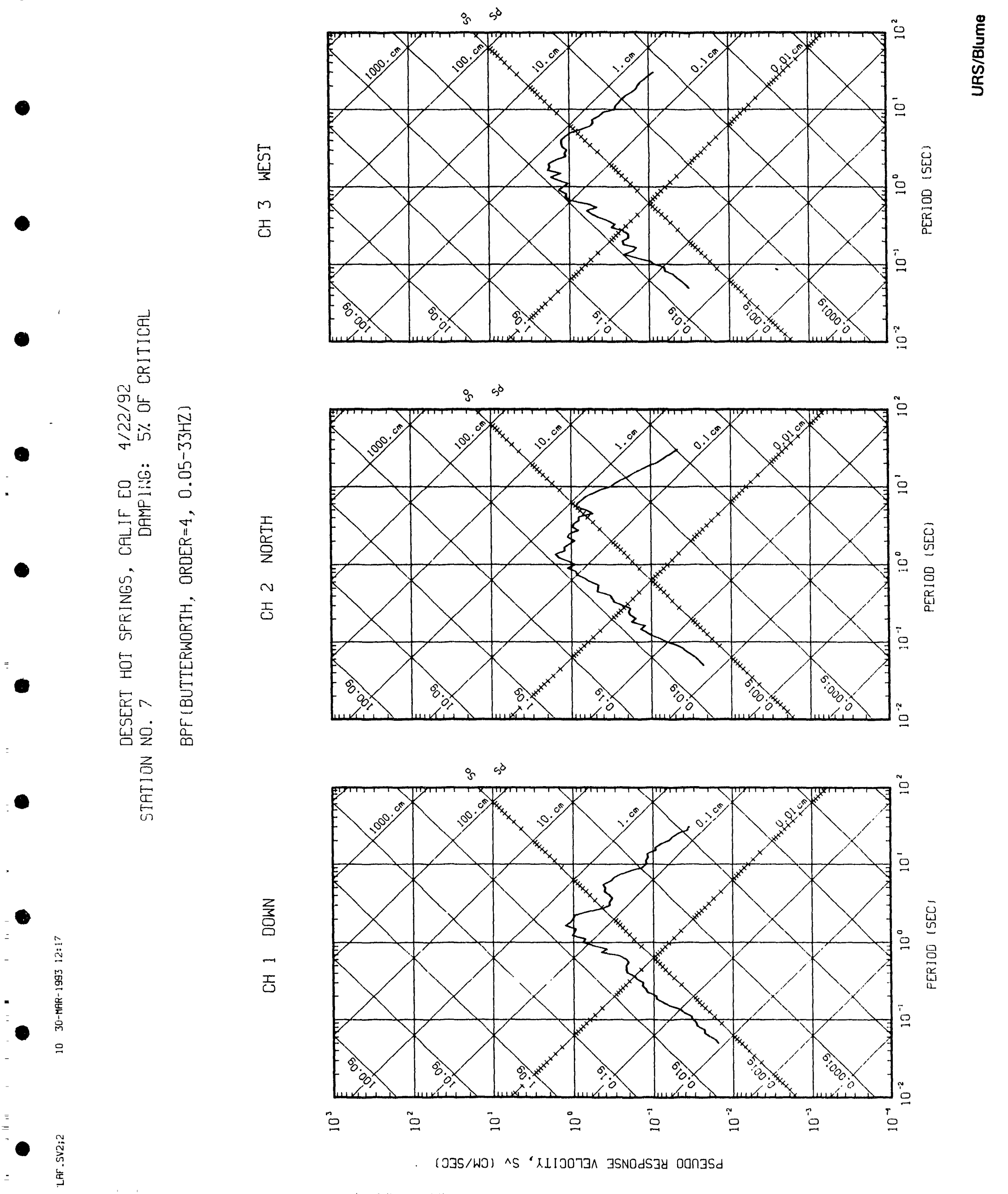


$\sum_{\infty}^{\frac{\pi}{\infty}}$

$\infty$

은

-

O

F

$\stackrel{5}{\omega}$

$=$ 


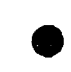

$\bullet$

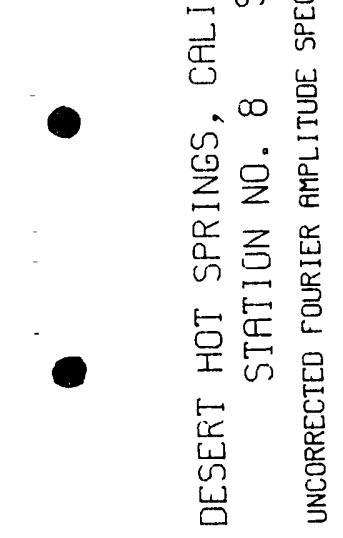

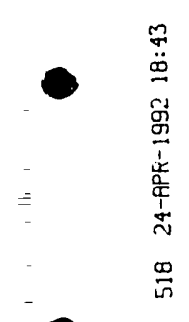

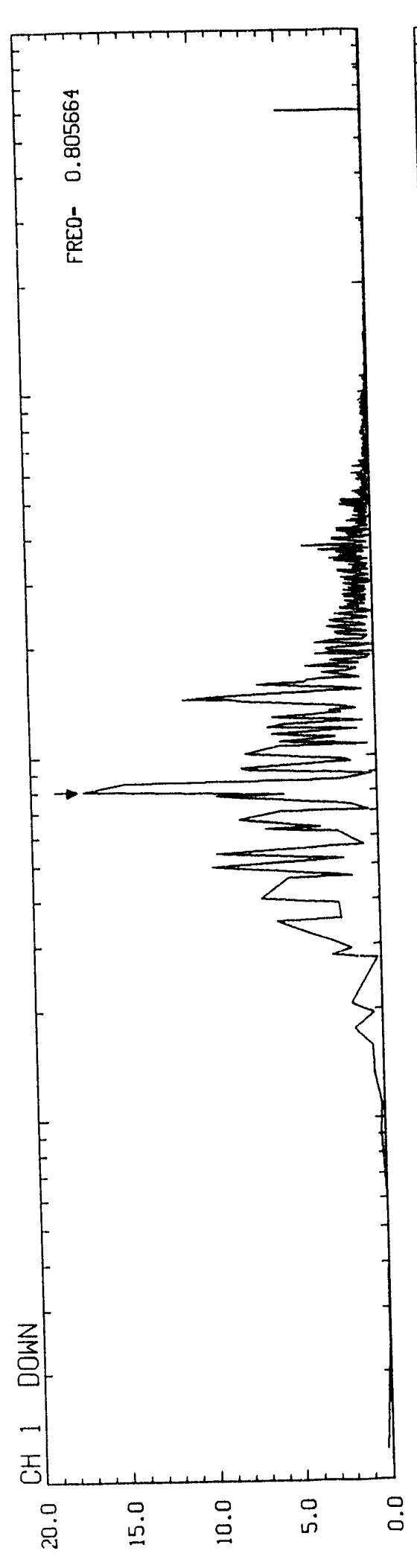

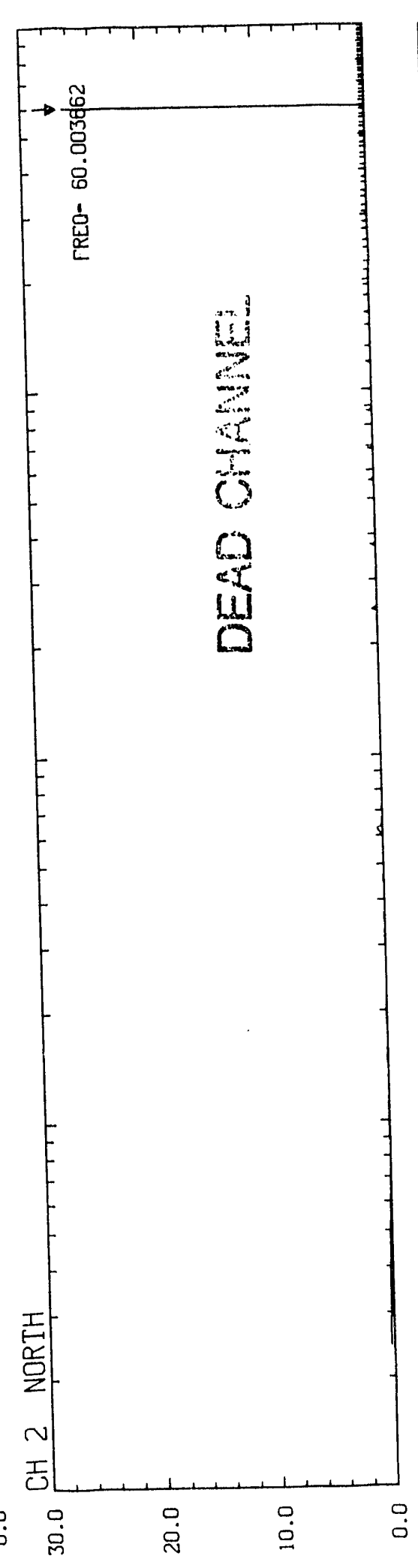

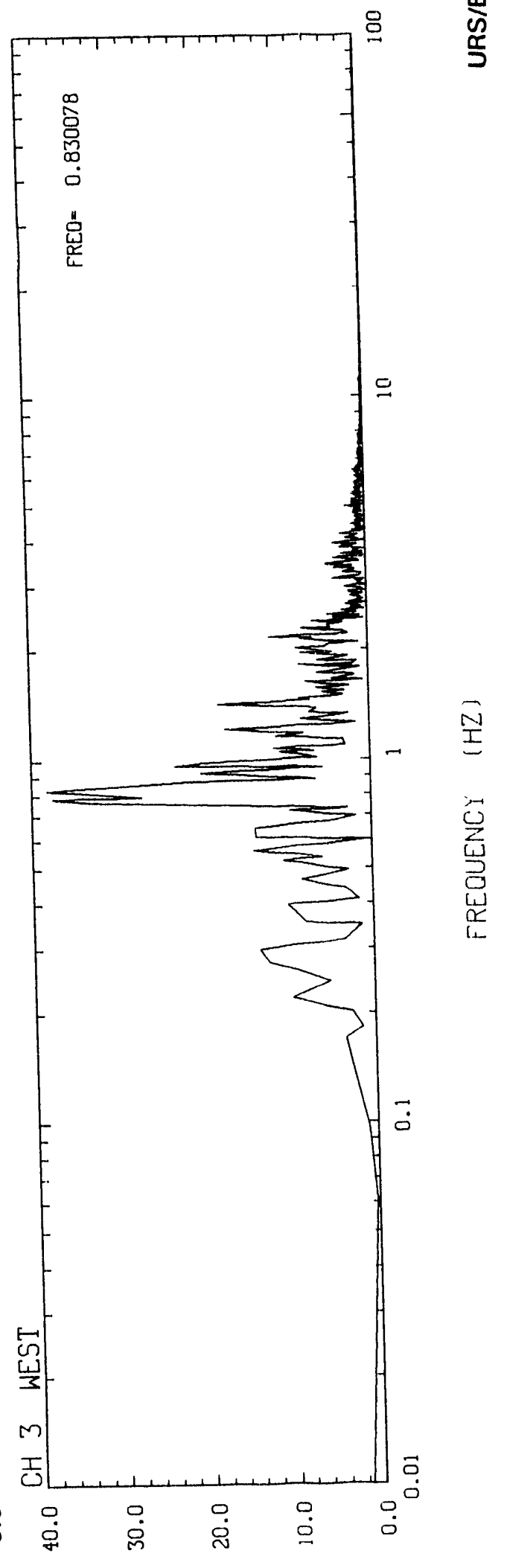

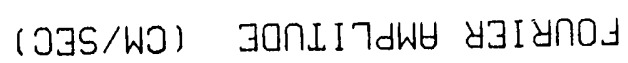



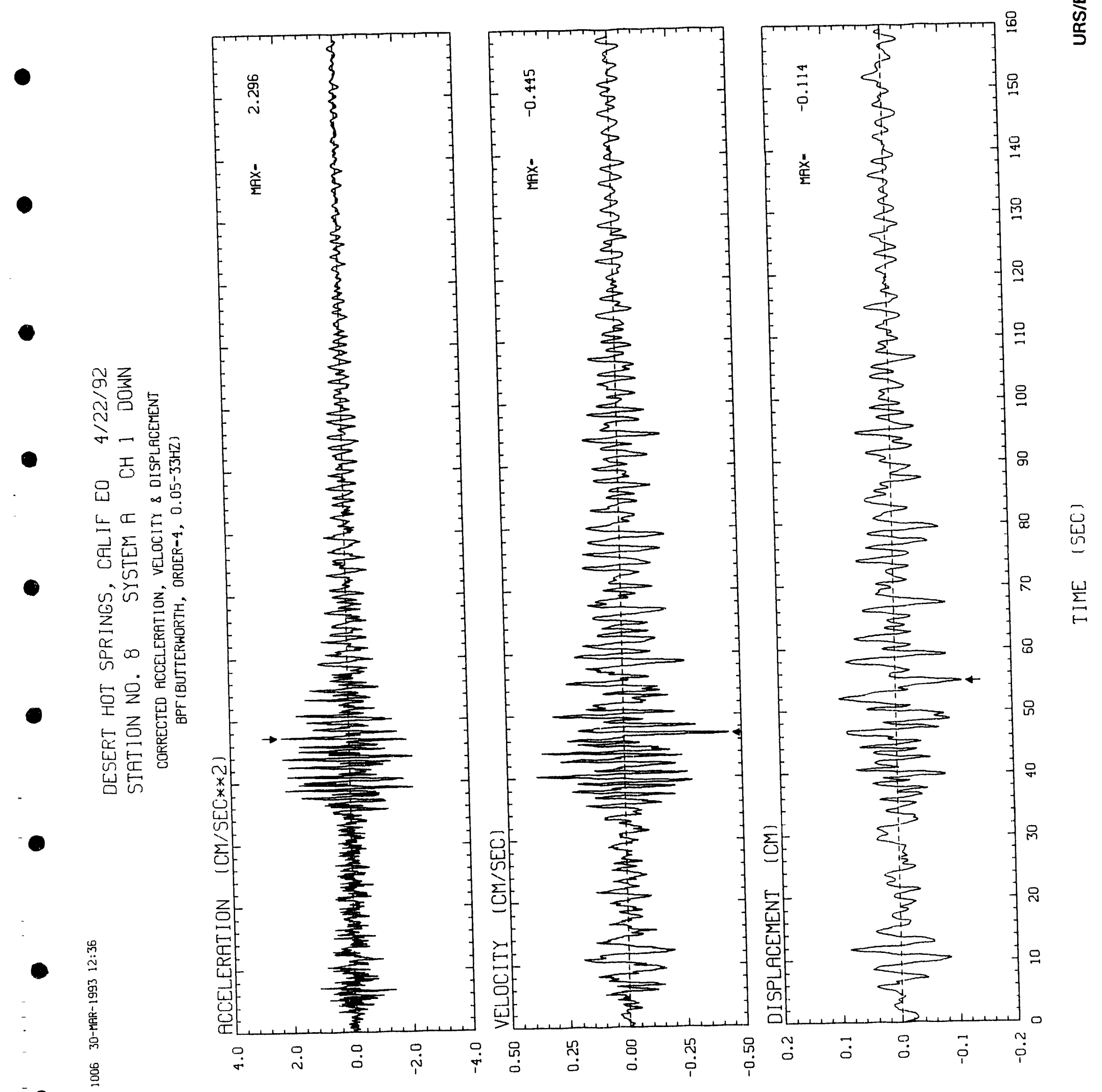

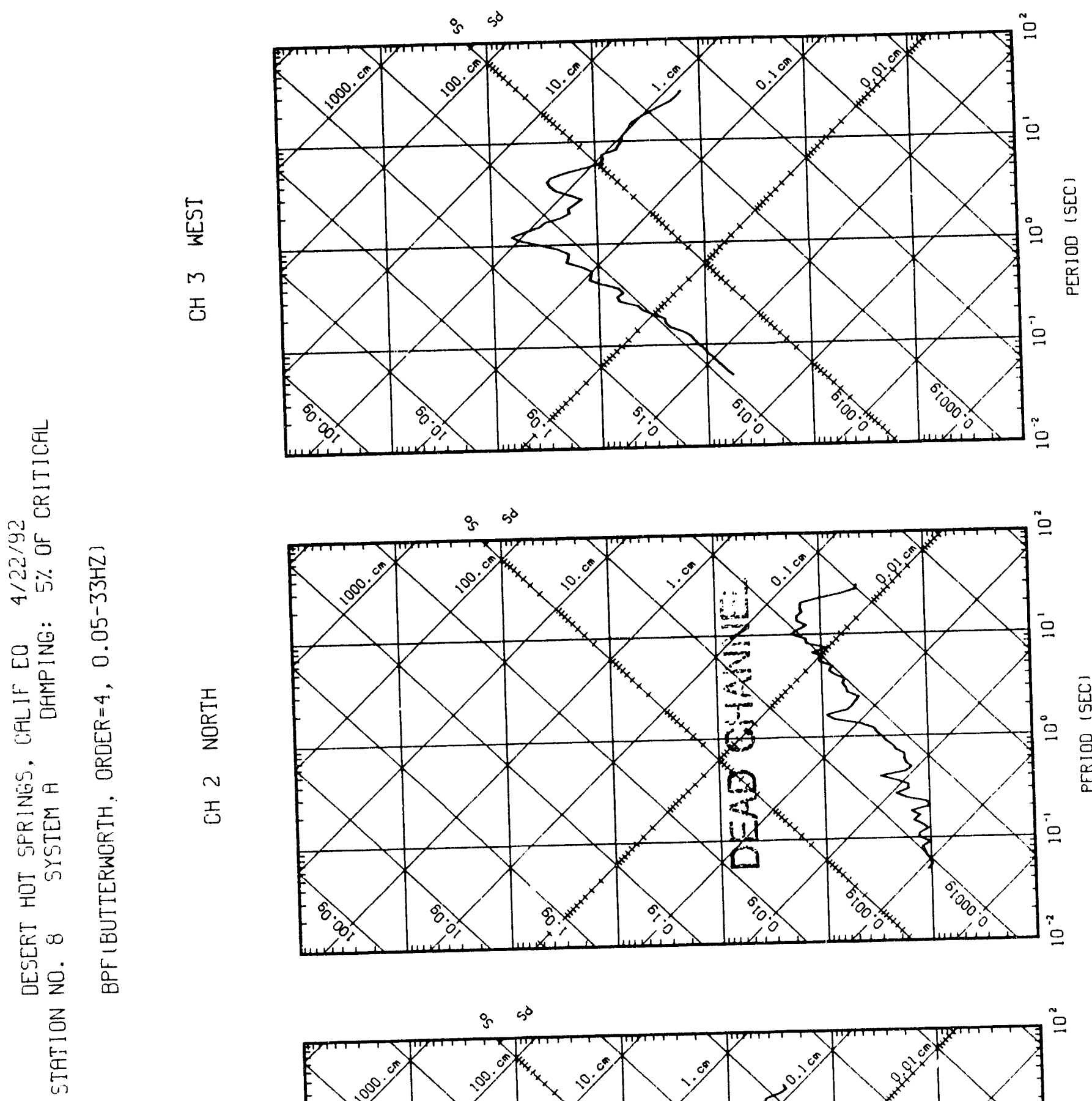

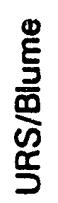
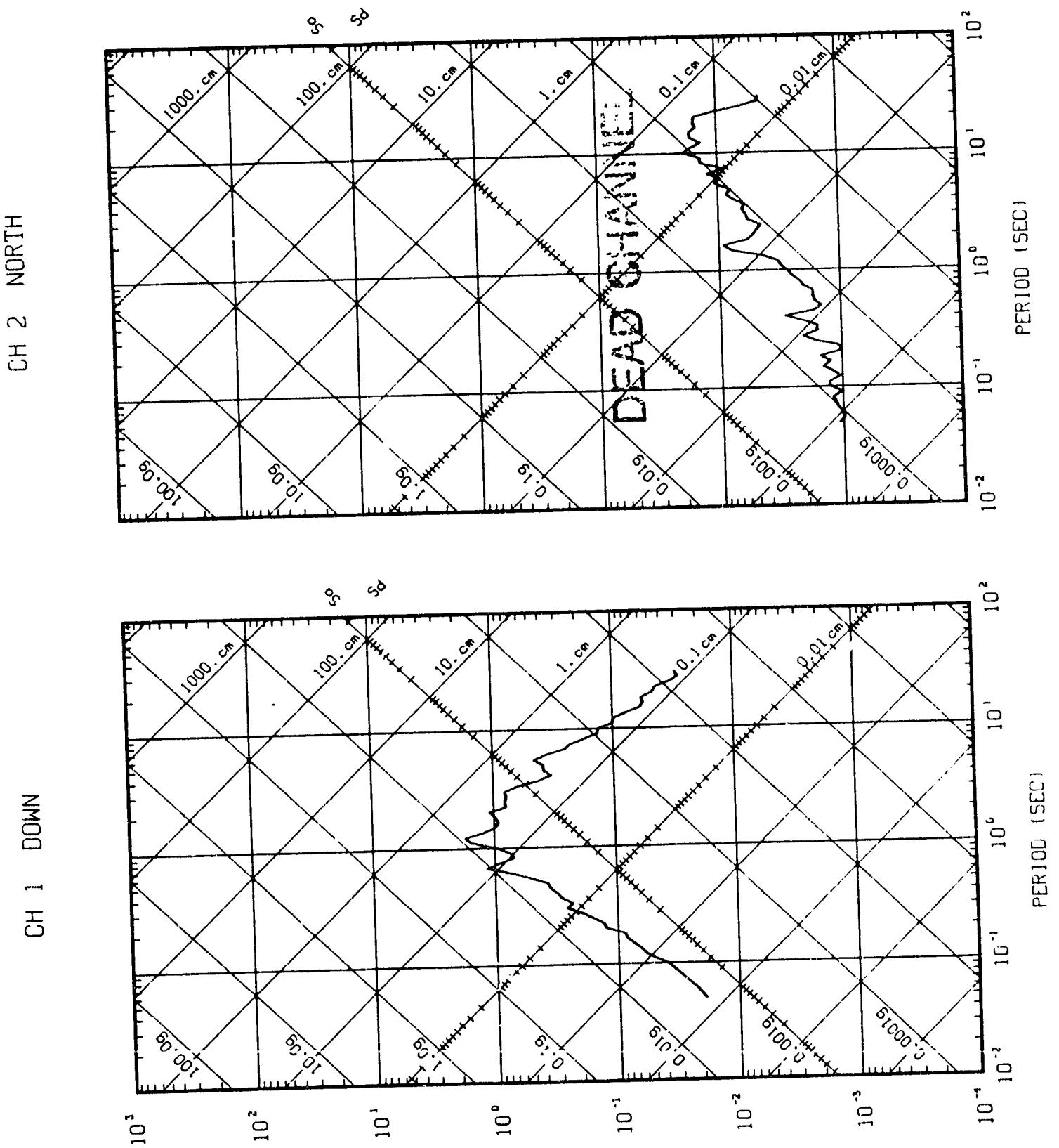

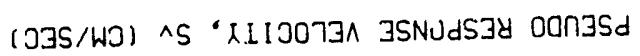


i 

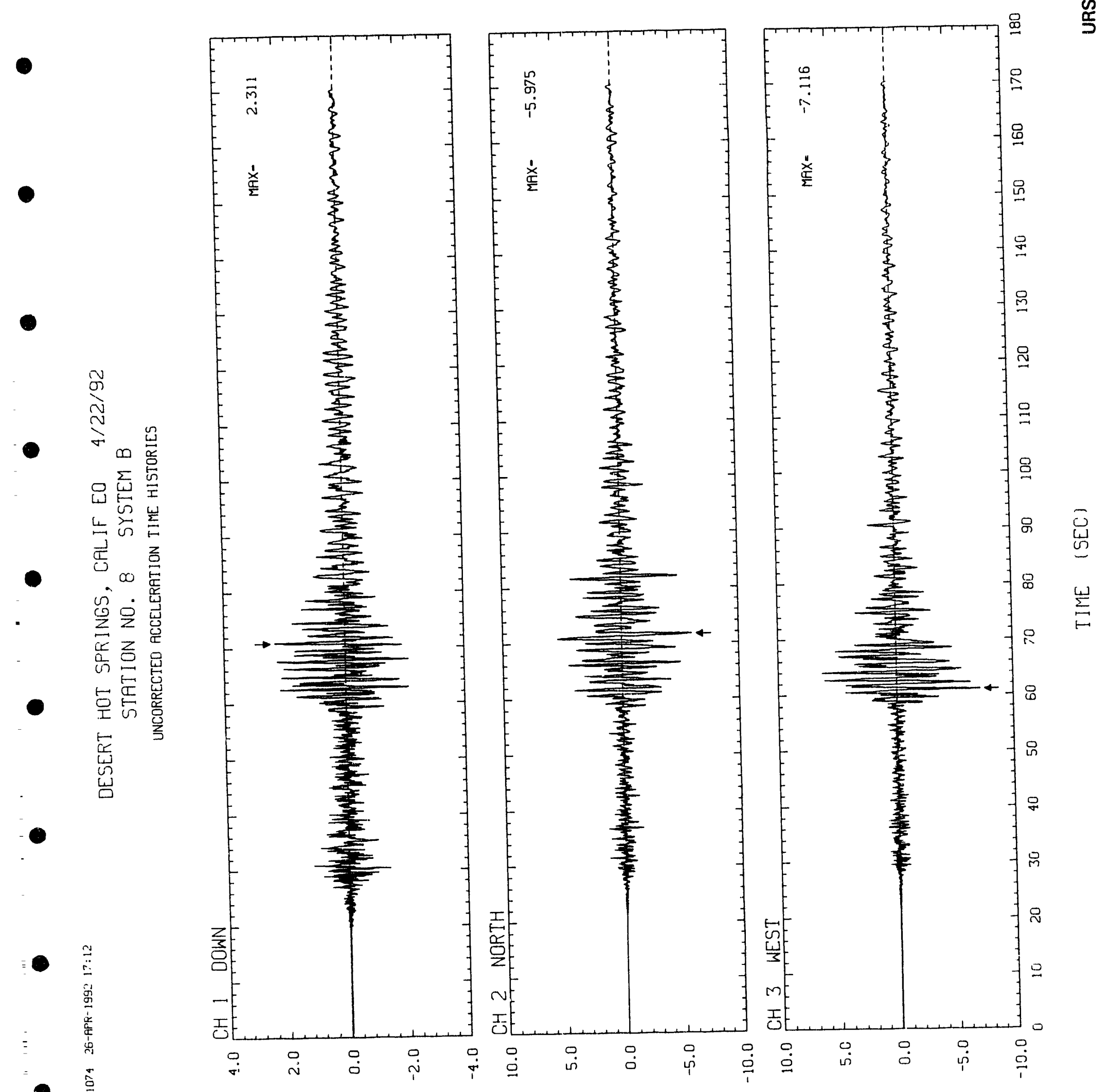

(2**JコS/WJ) NOI I甘4 $37 \exists J 34$ 


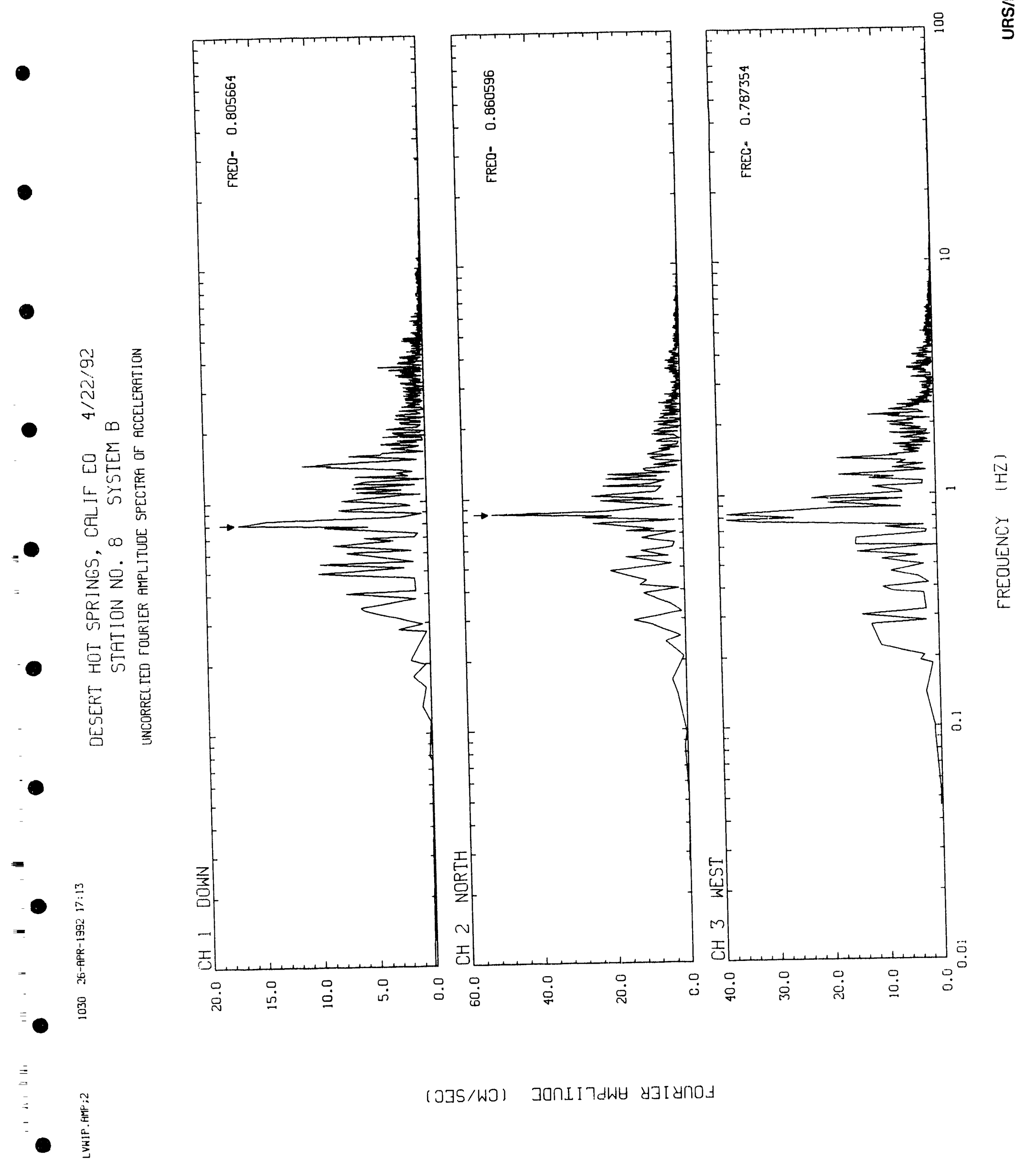




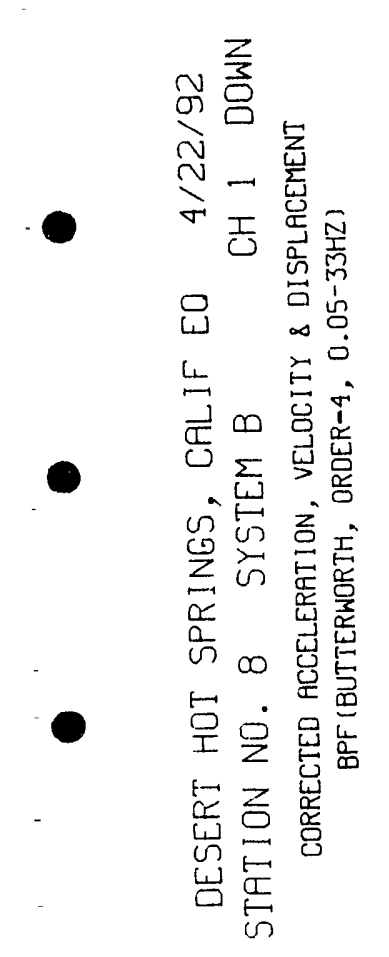

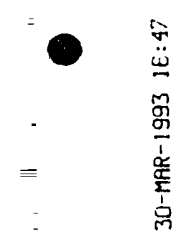

-

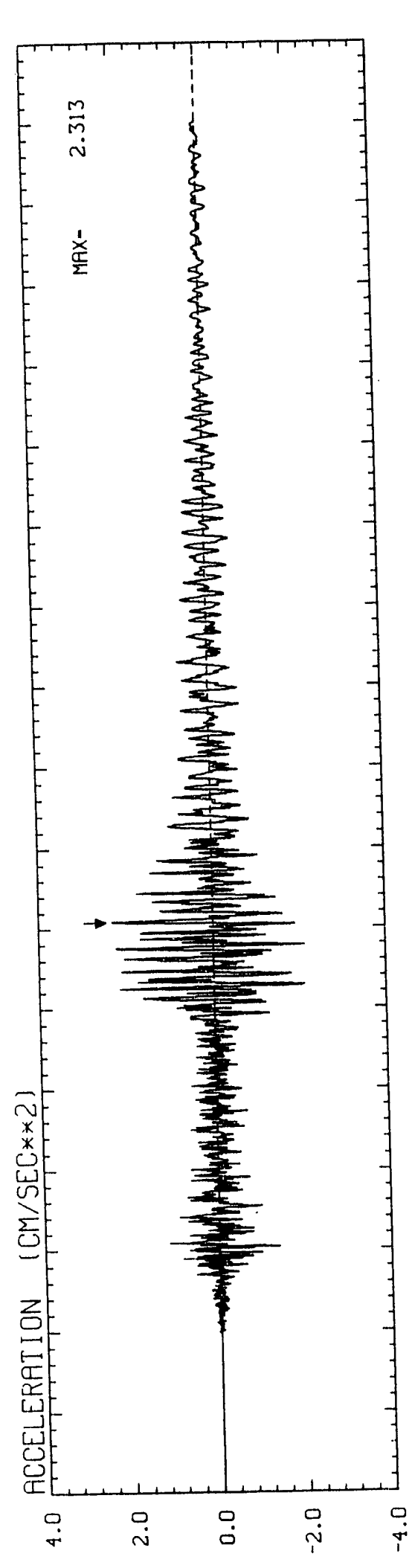

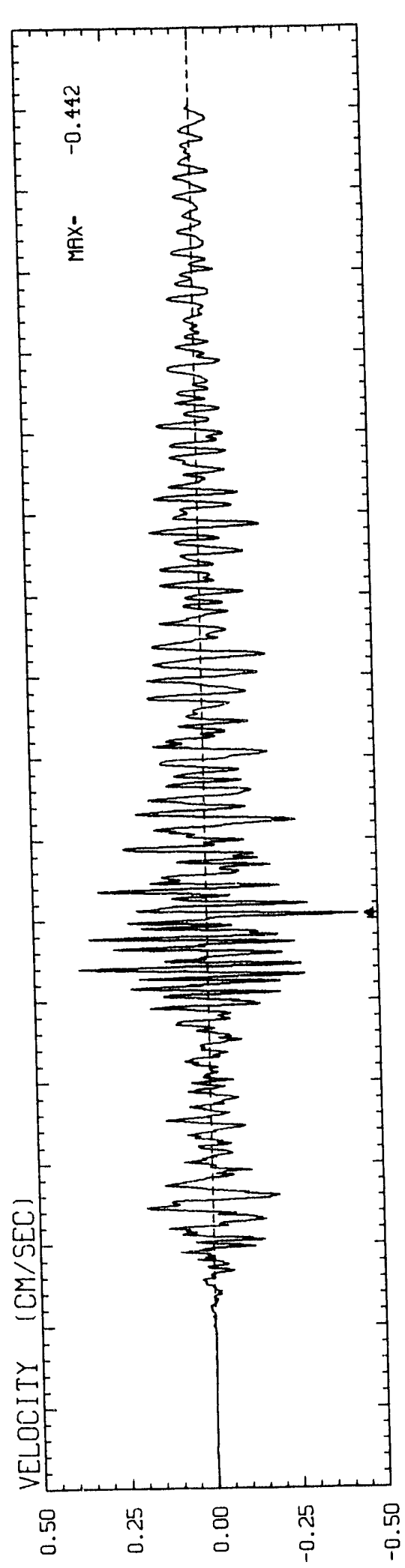

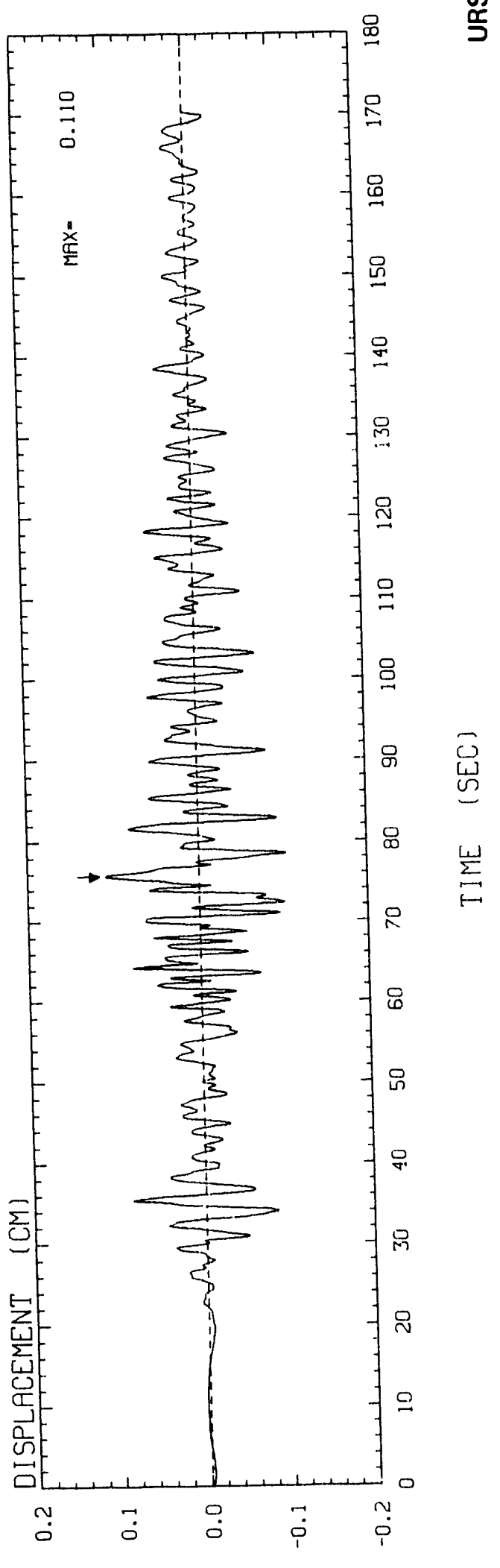



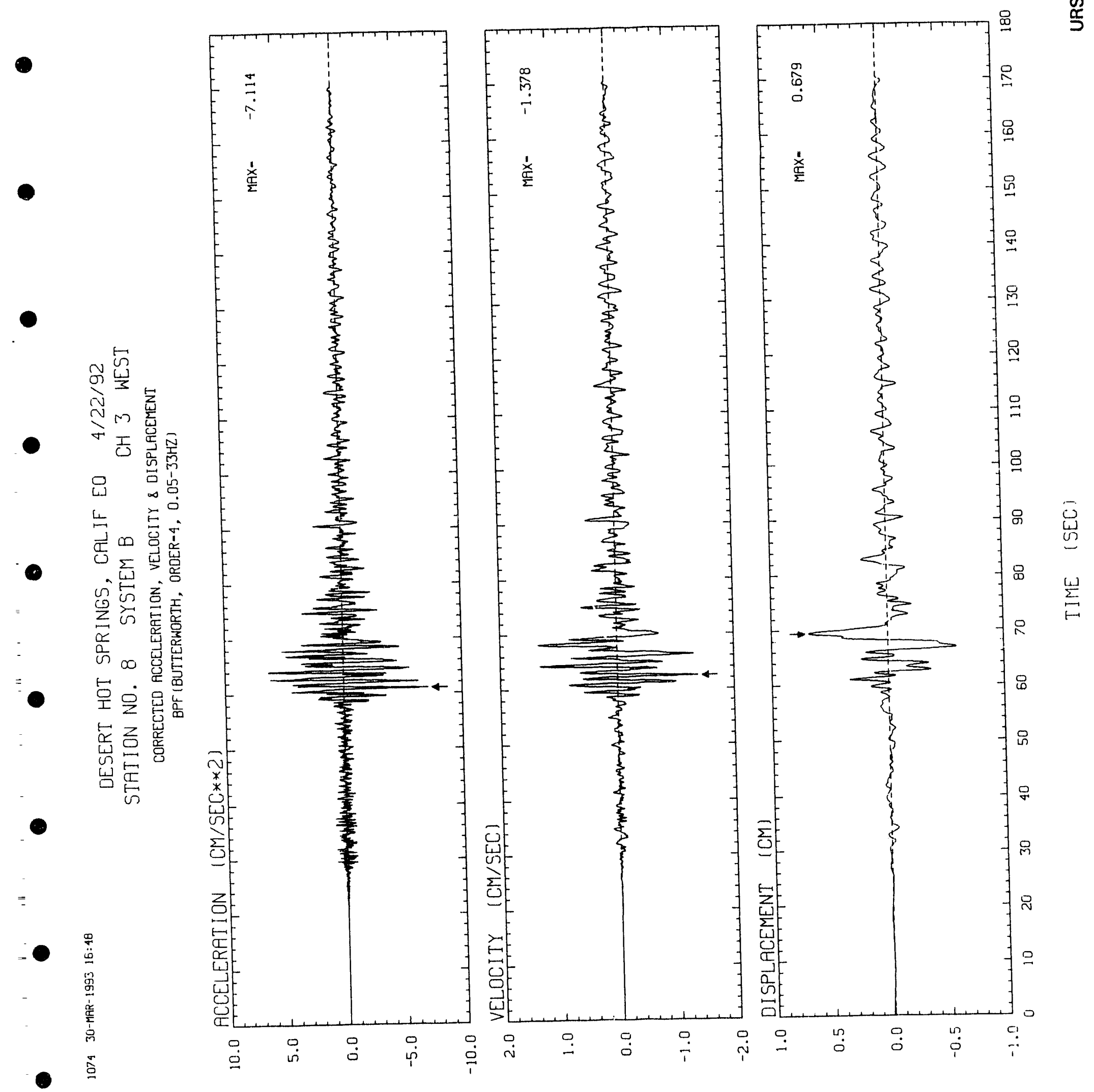

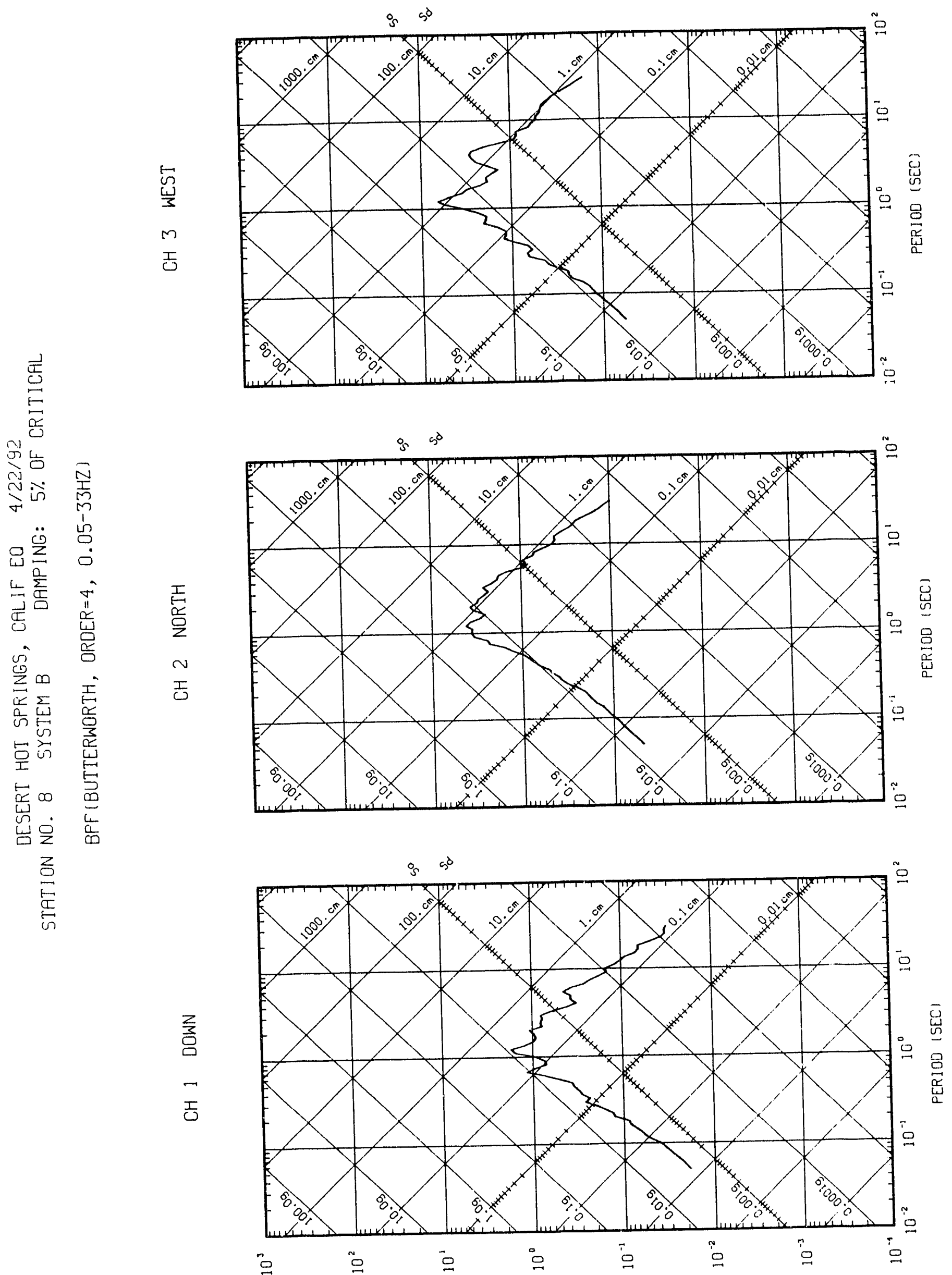

(J3S/WO) ^S 'XIIJOTJA JSNOdSJY OONJSd 

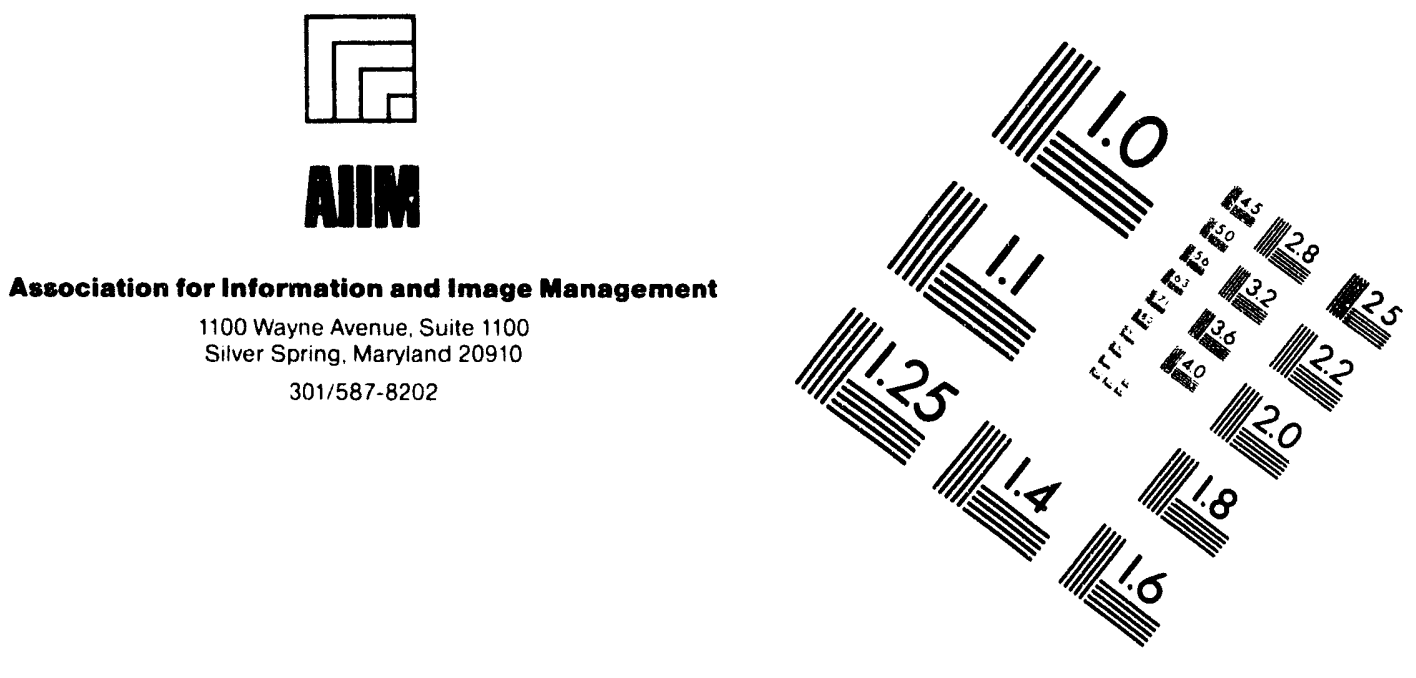

\section{Centimeter}

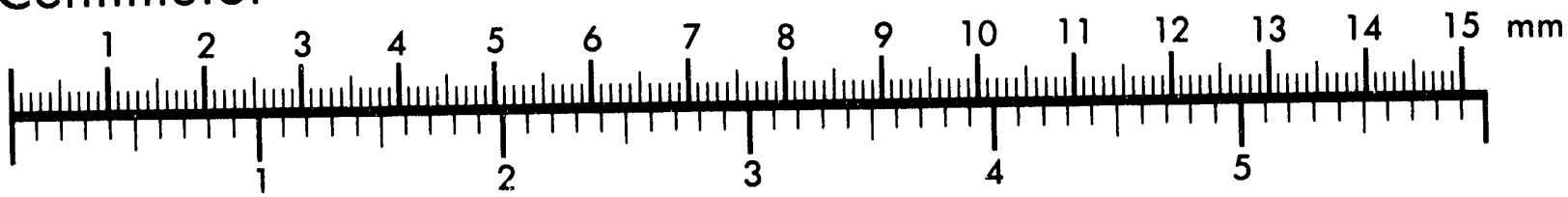
Inches
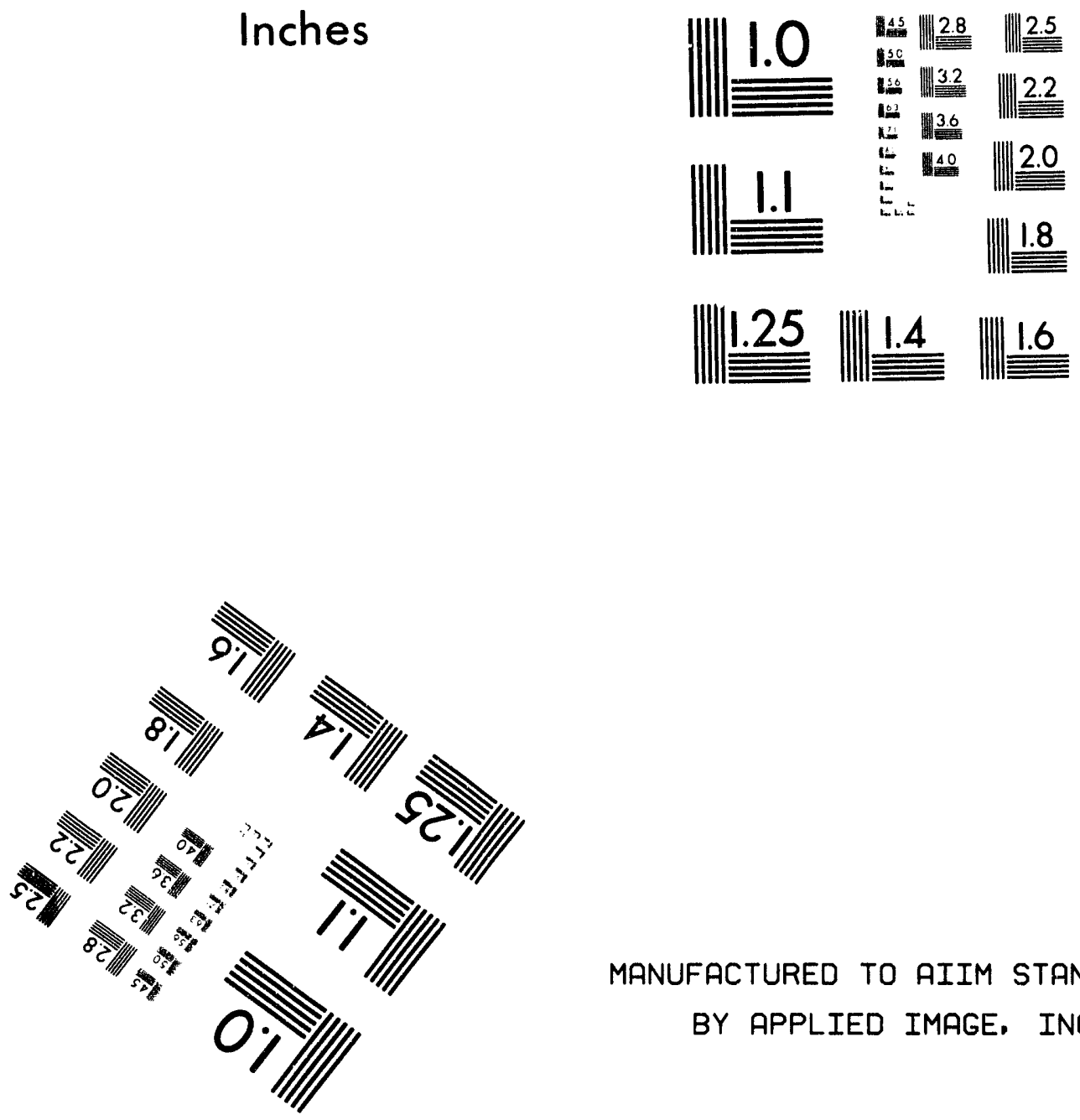

MANUFACTURED TO AIIM STANDARDS

BY APPLIED IMAGE, INC.

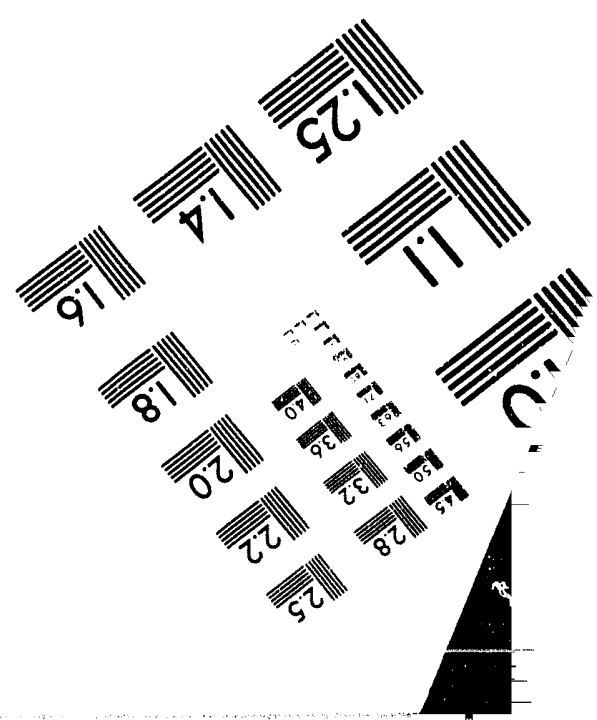



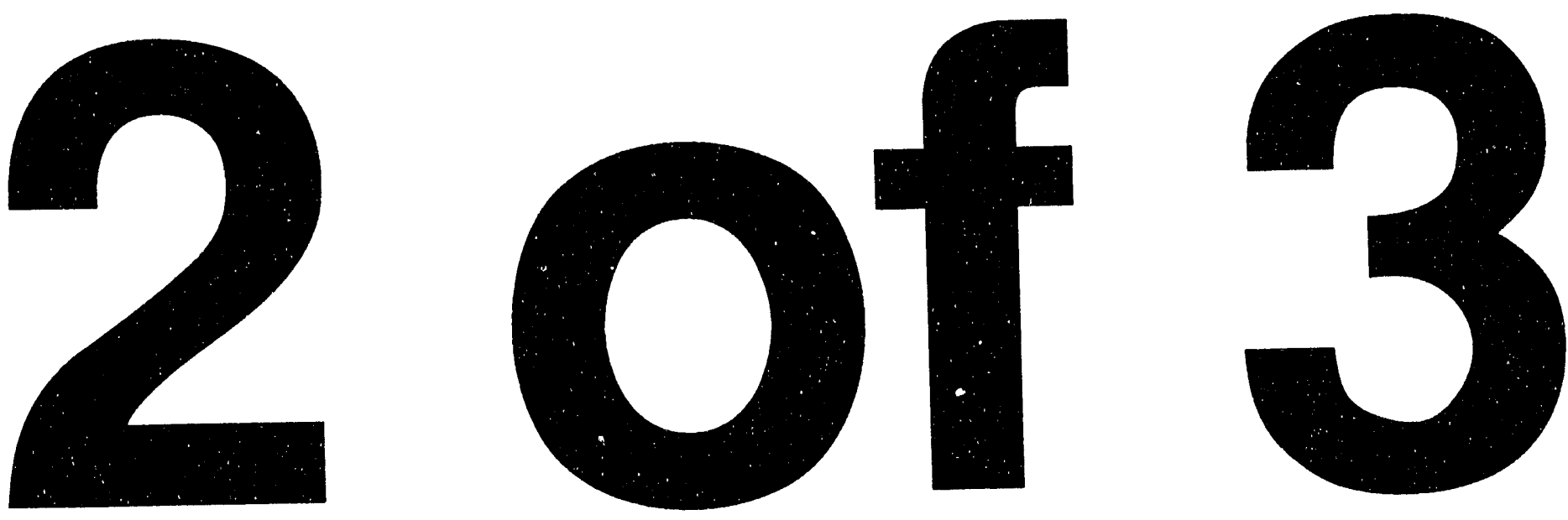


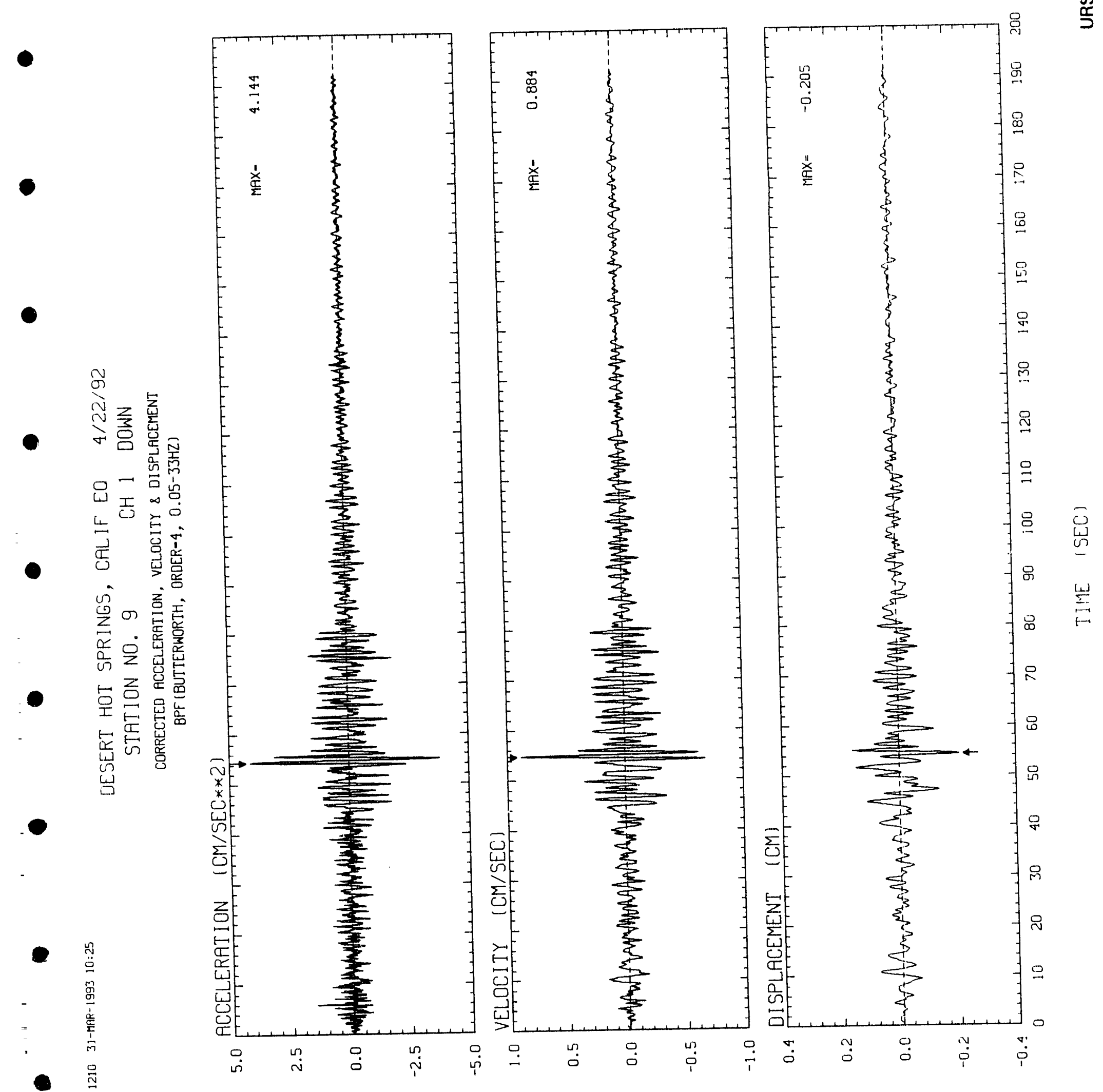




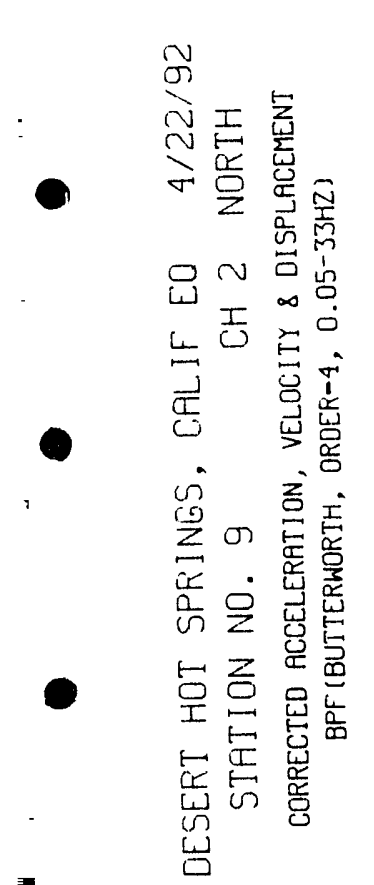

-

管

- 임
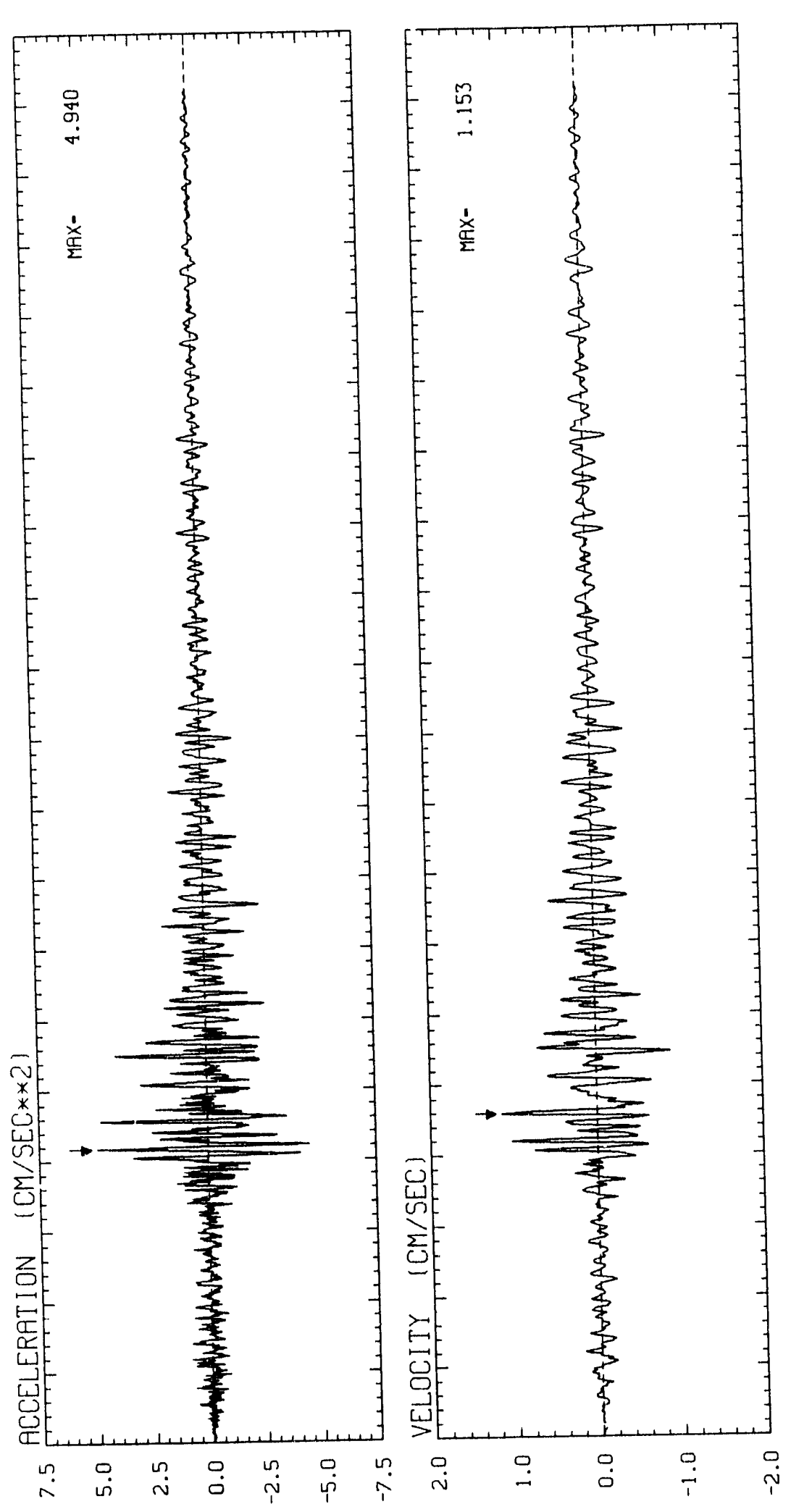

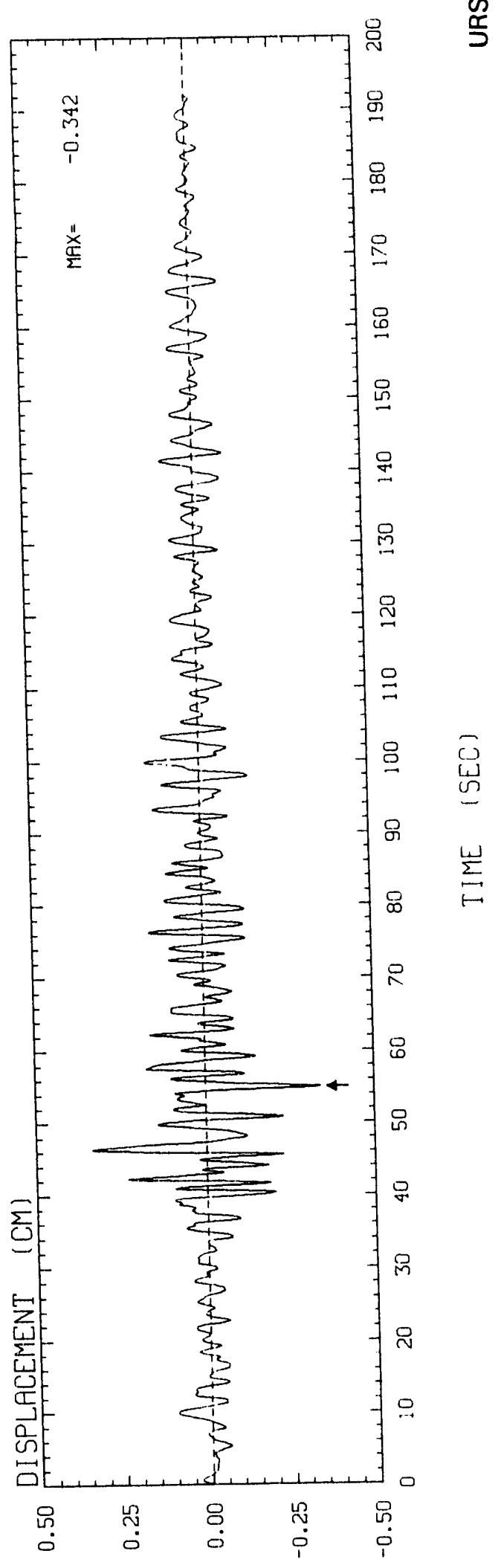

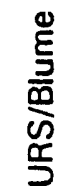




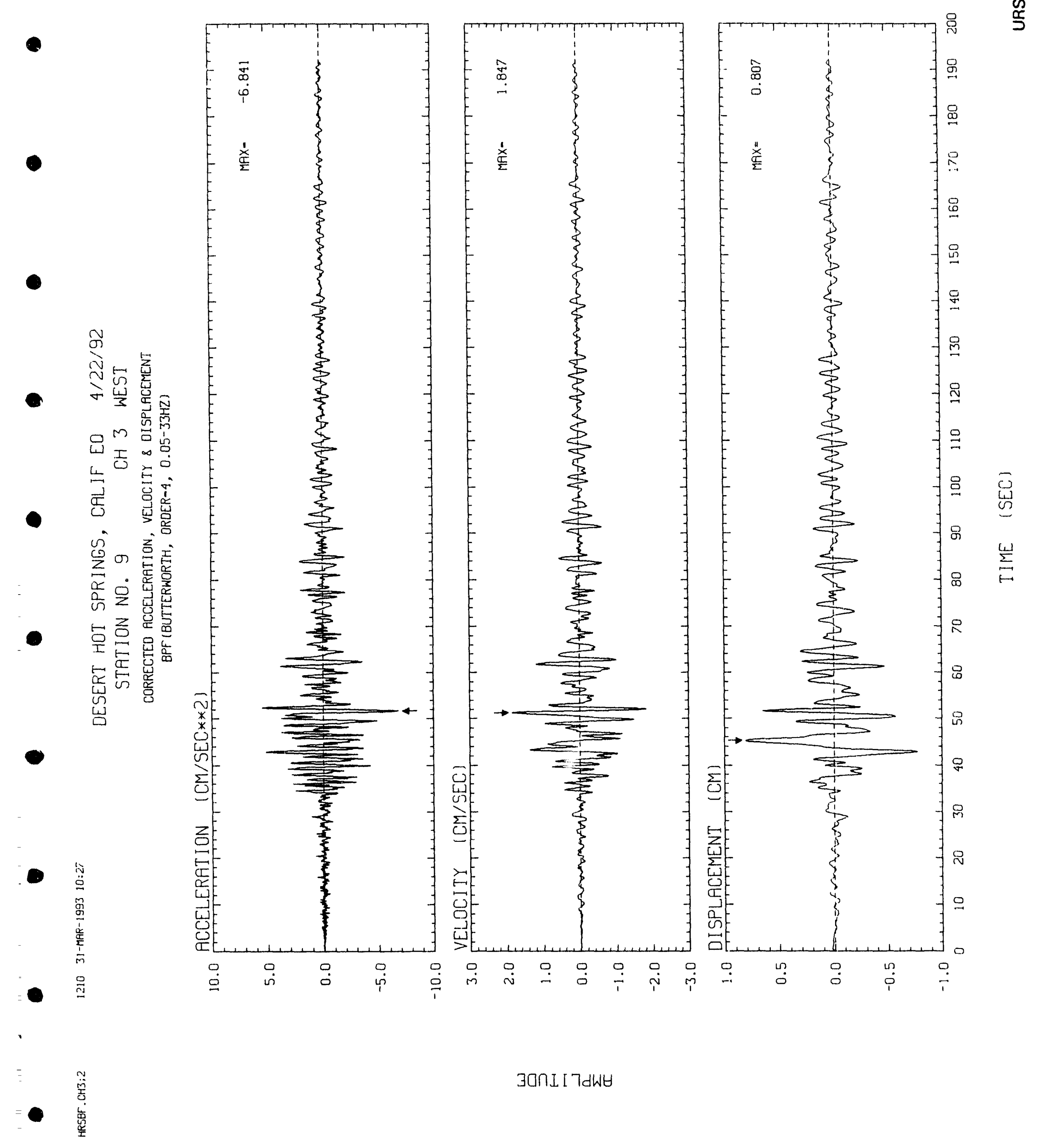




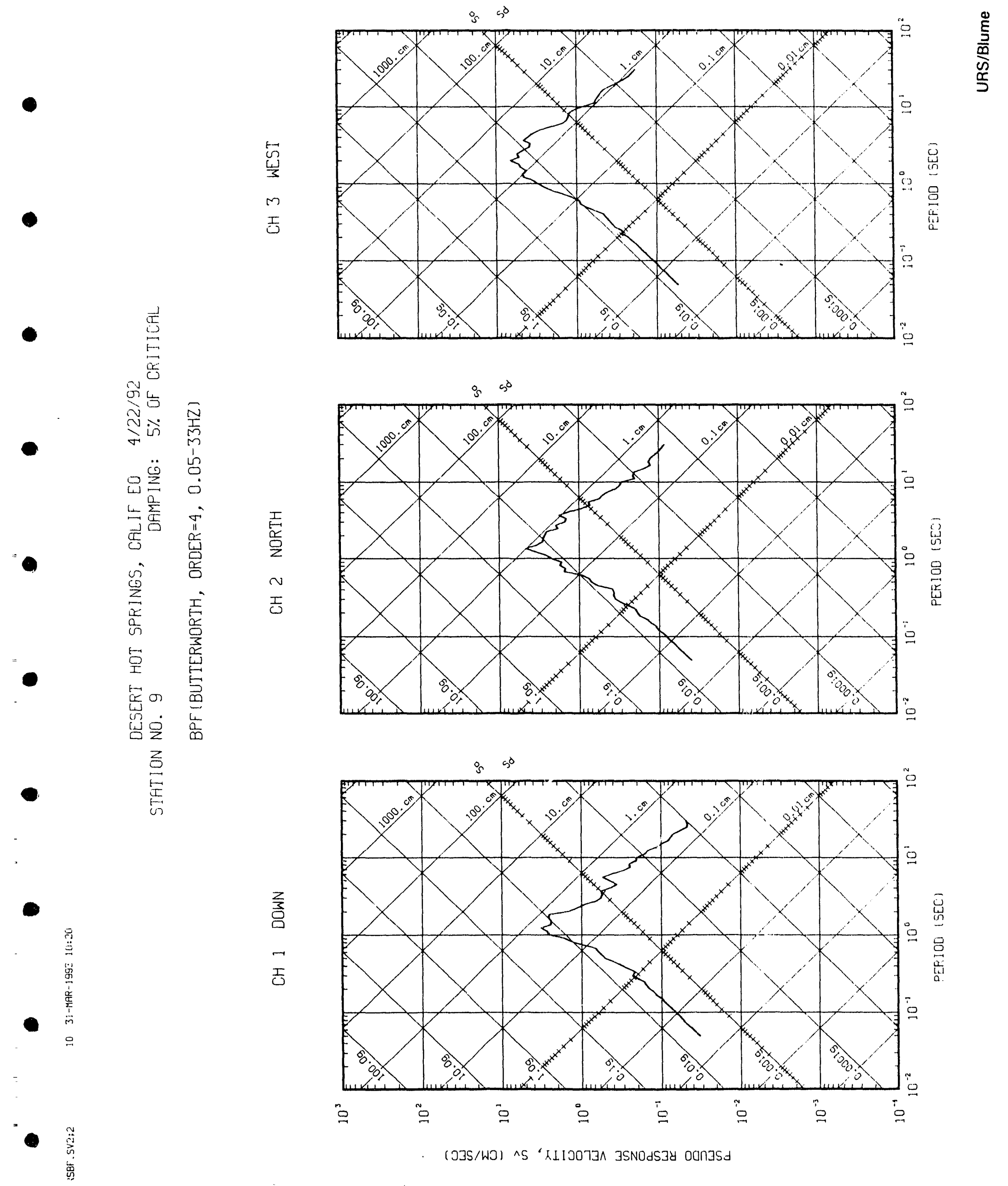




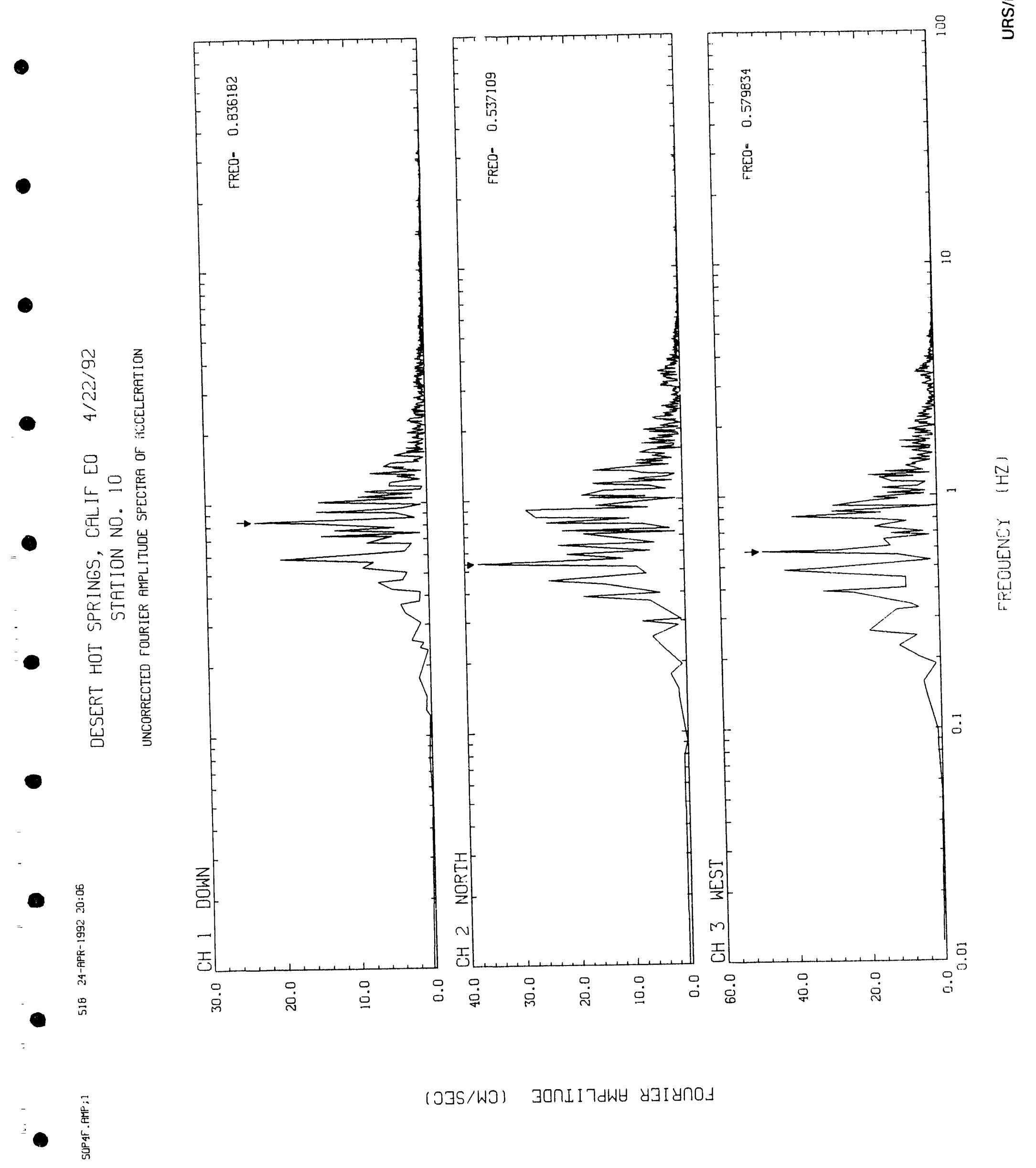



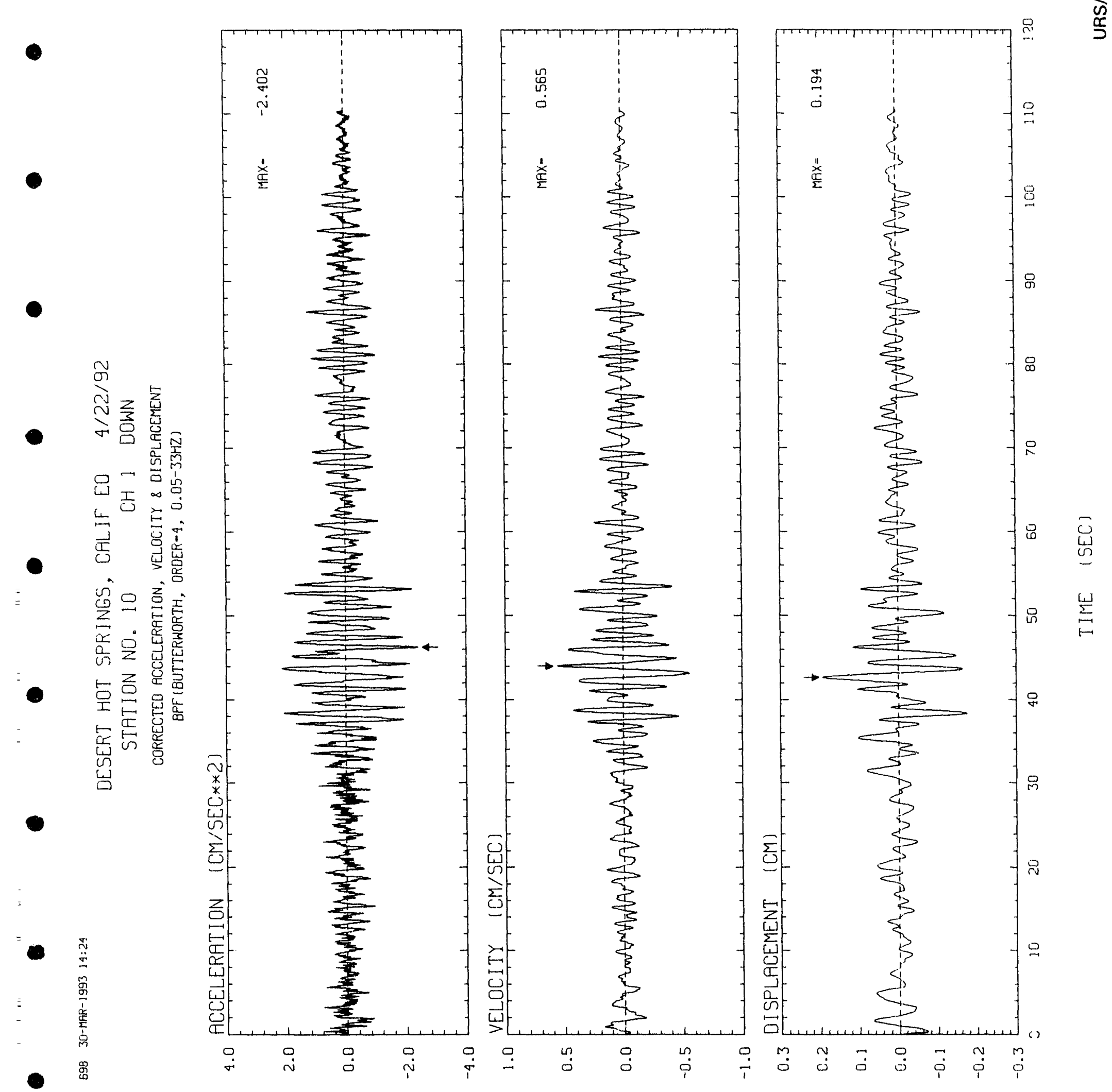

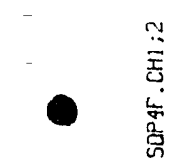




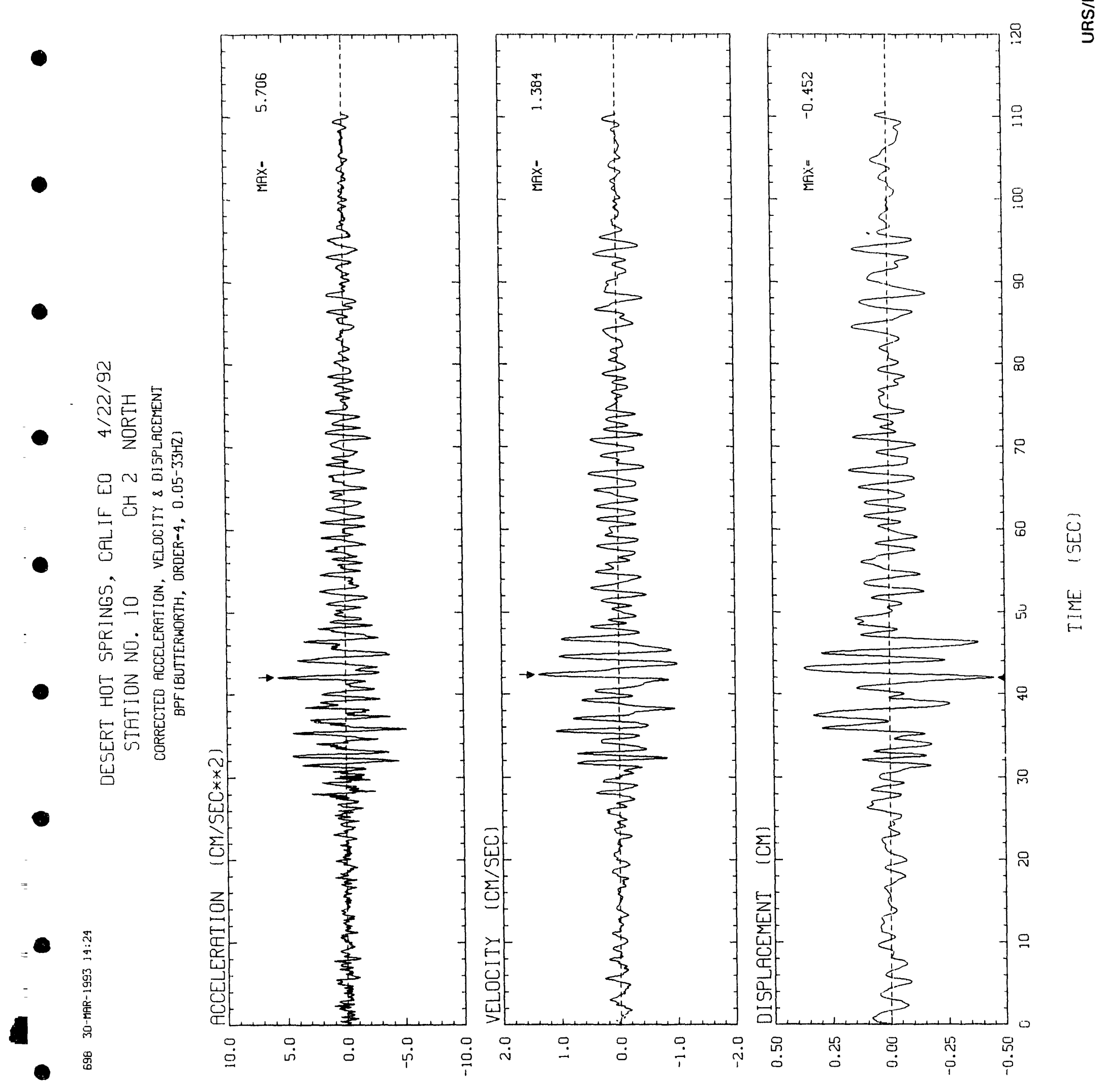




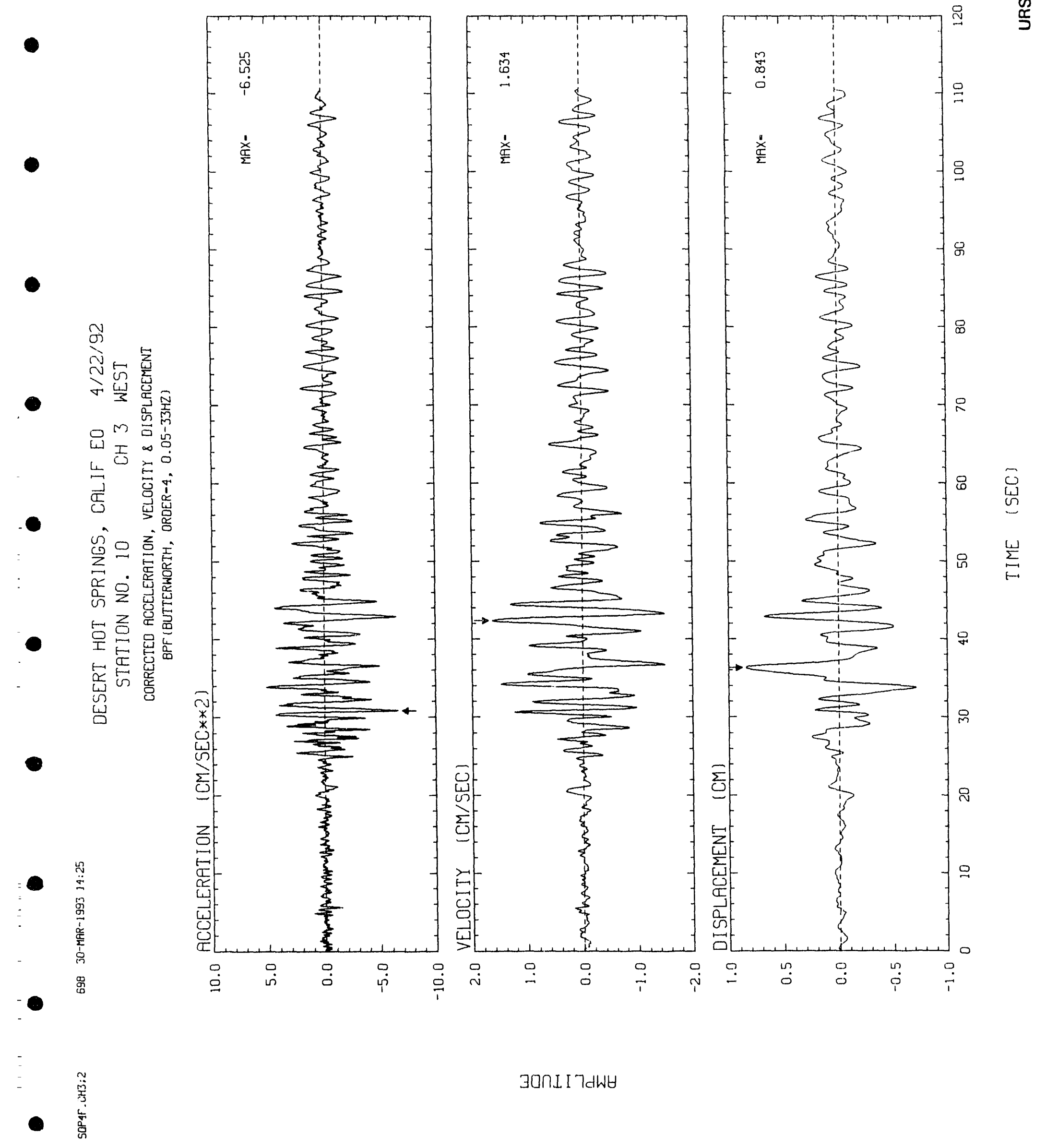



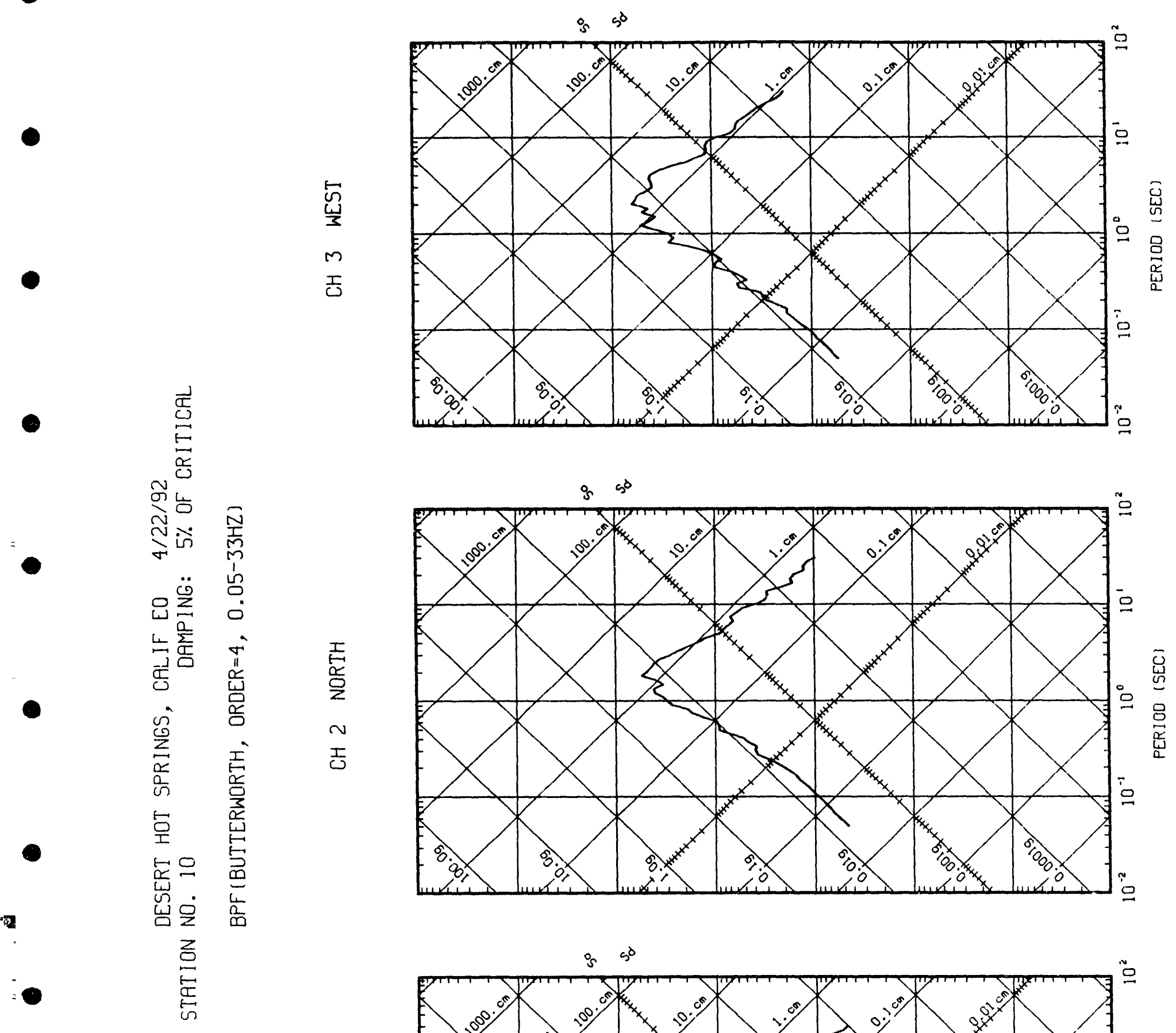

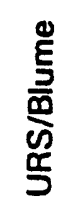
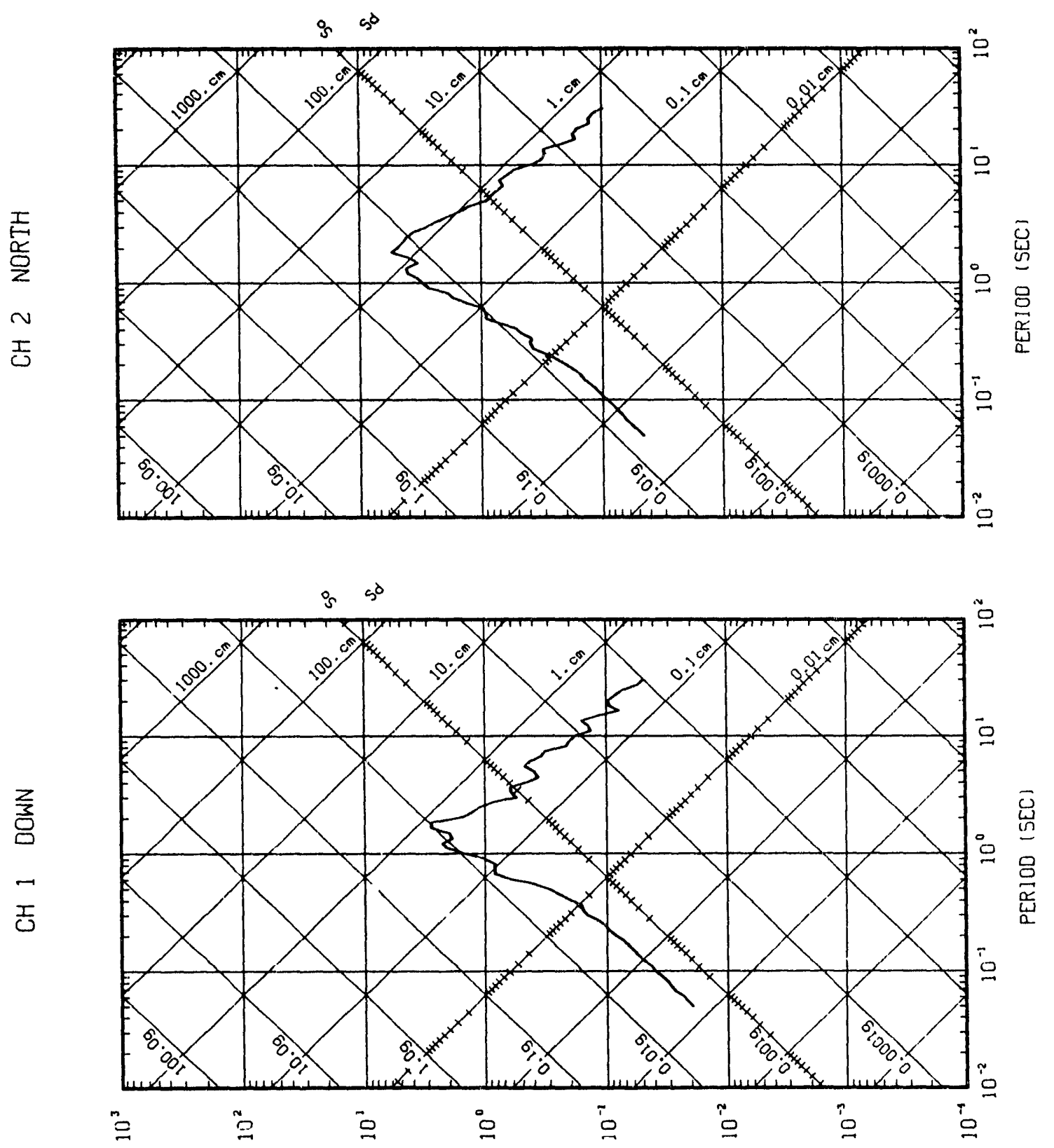

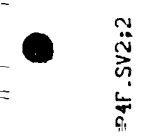


-

$r$

0

2

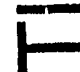

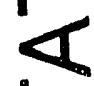

10

0

$=$ 


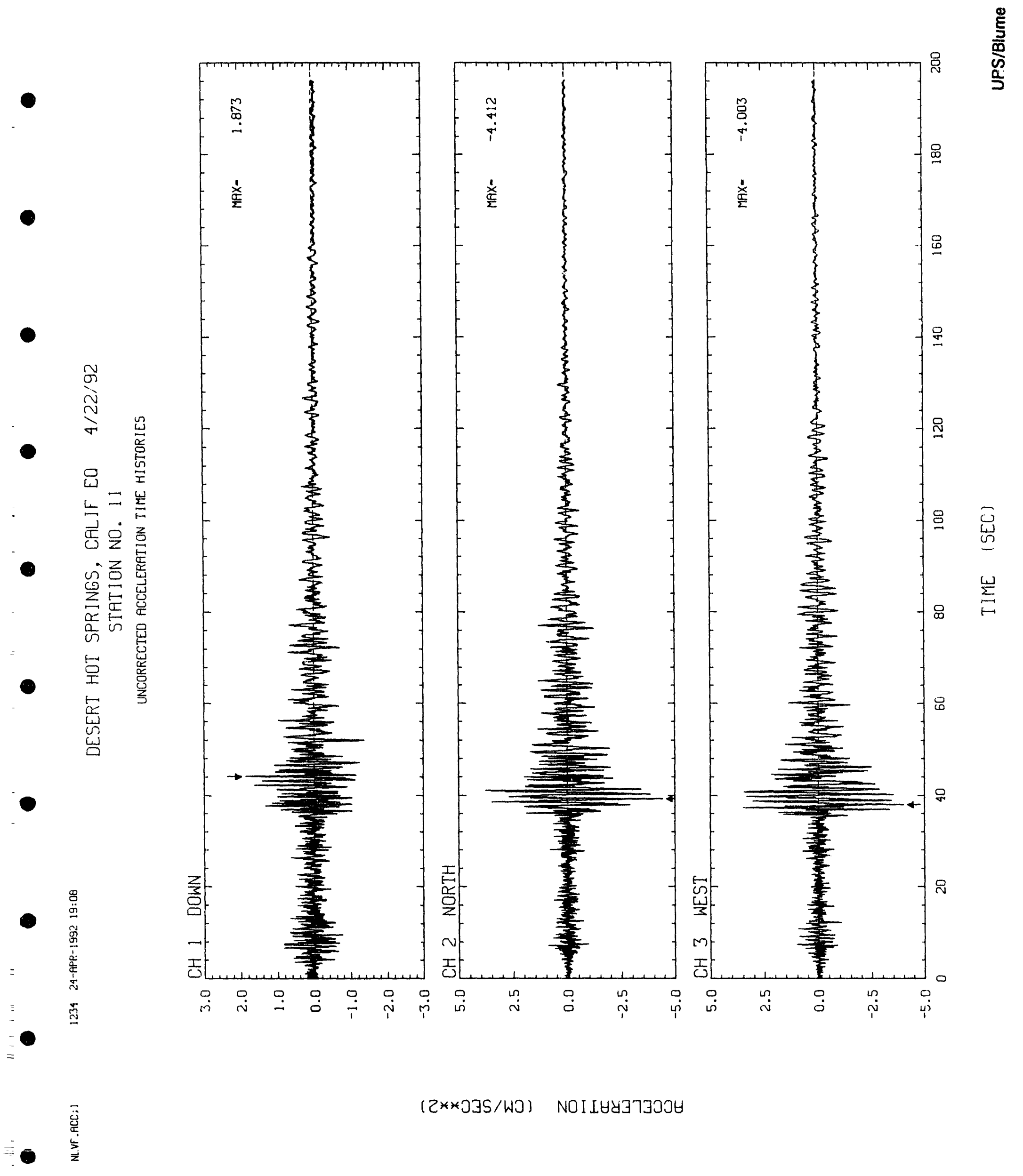



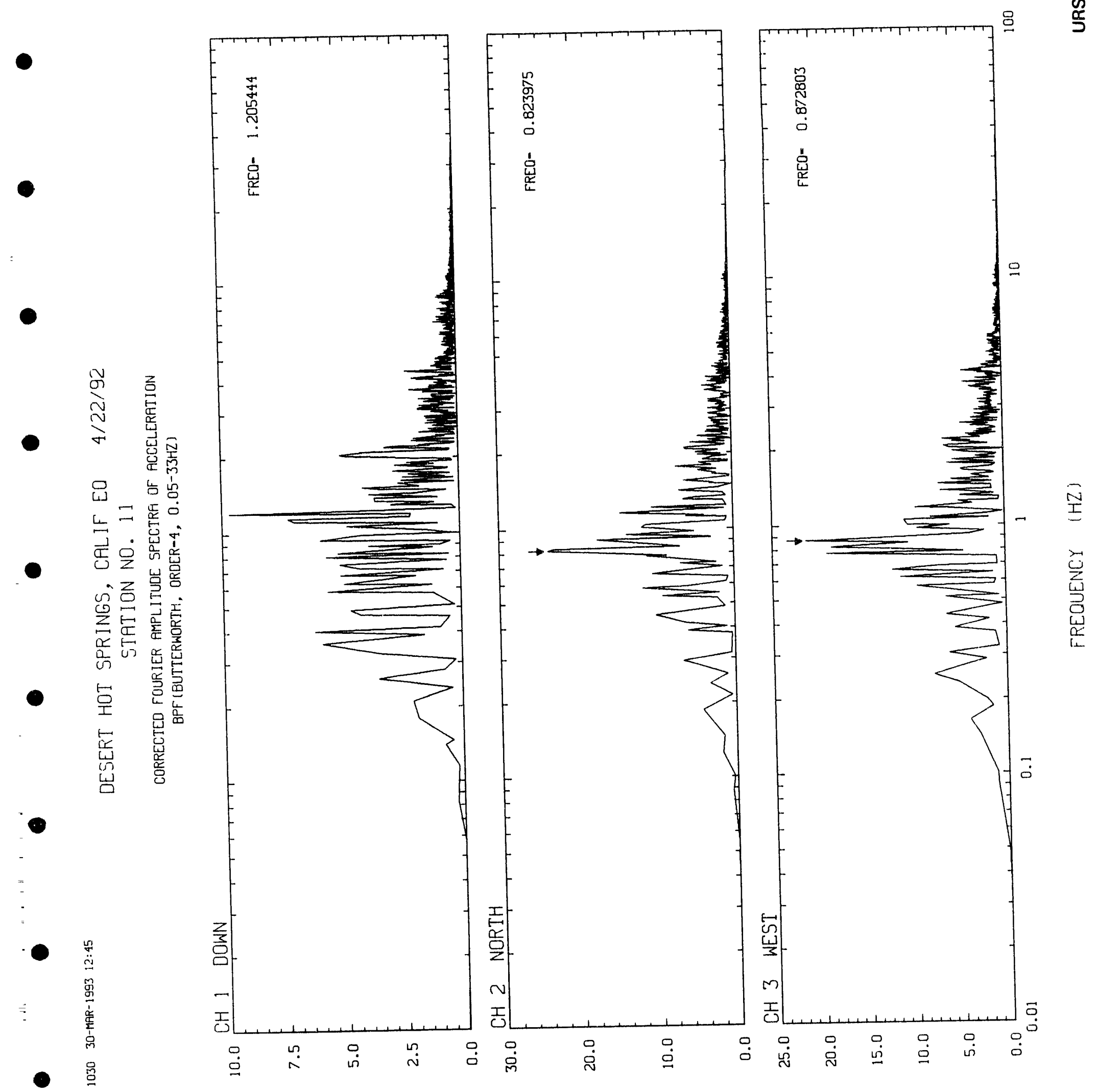

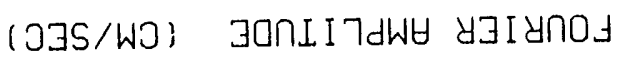



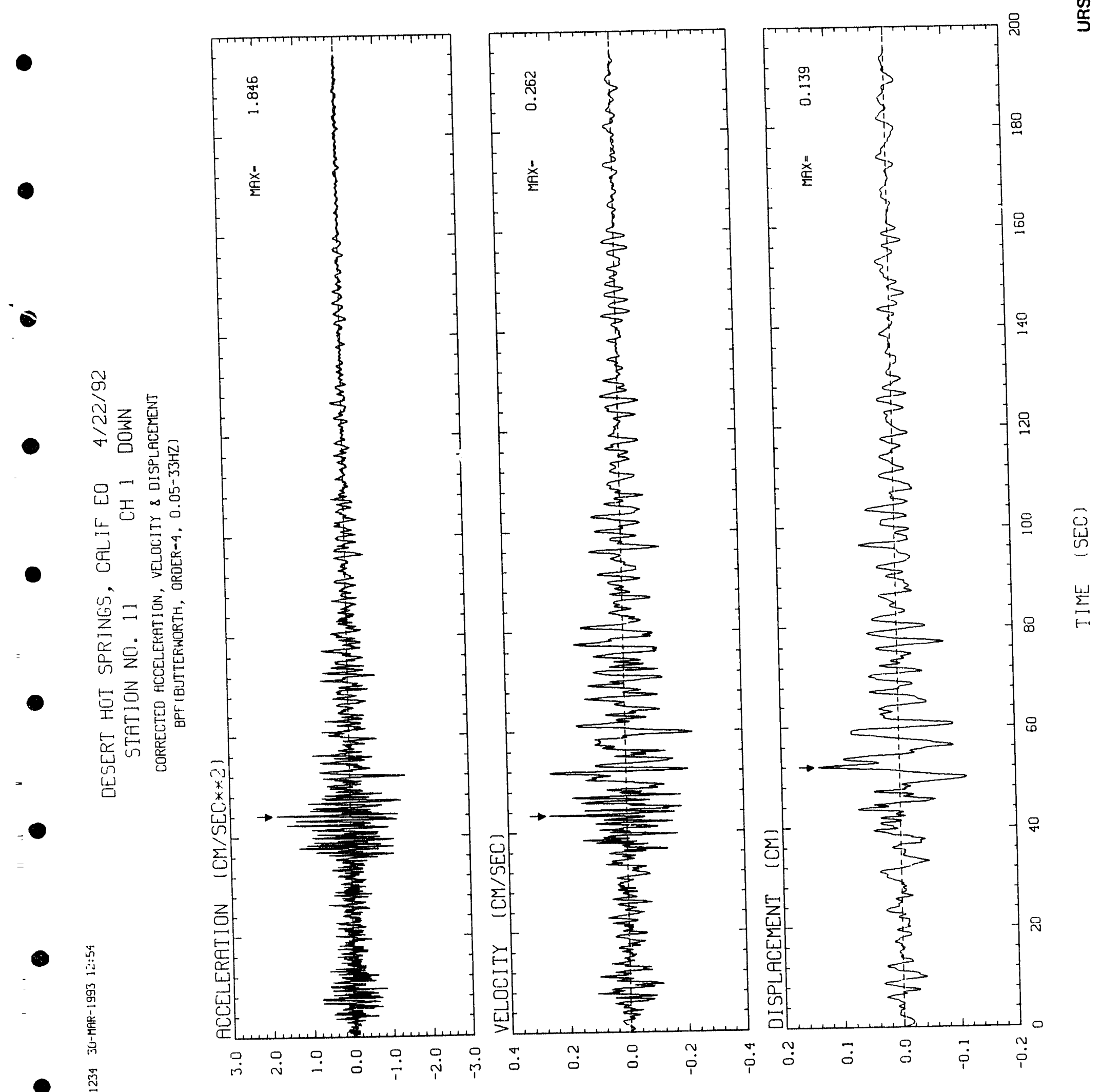

$-1 \quad \frac{?}{\vec{T}}$ 

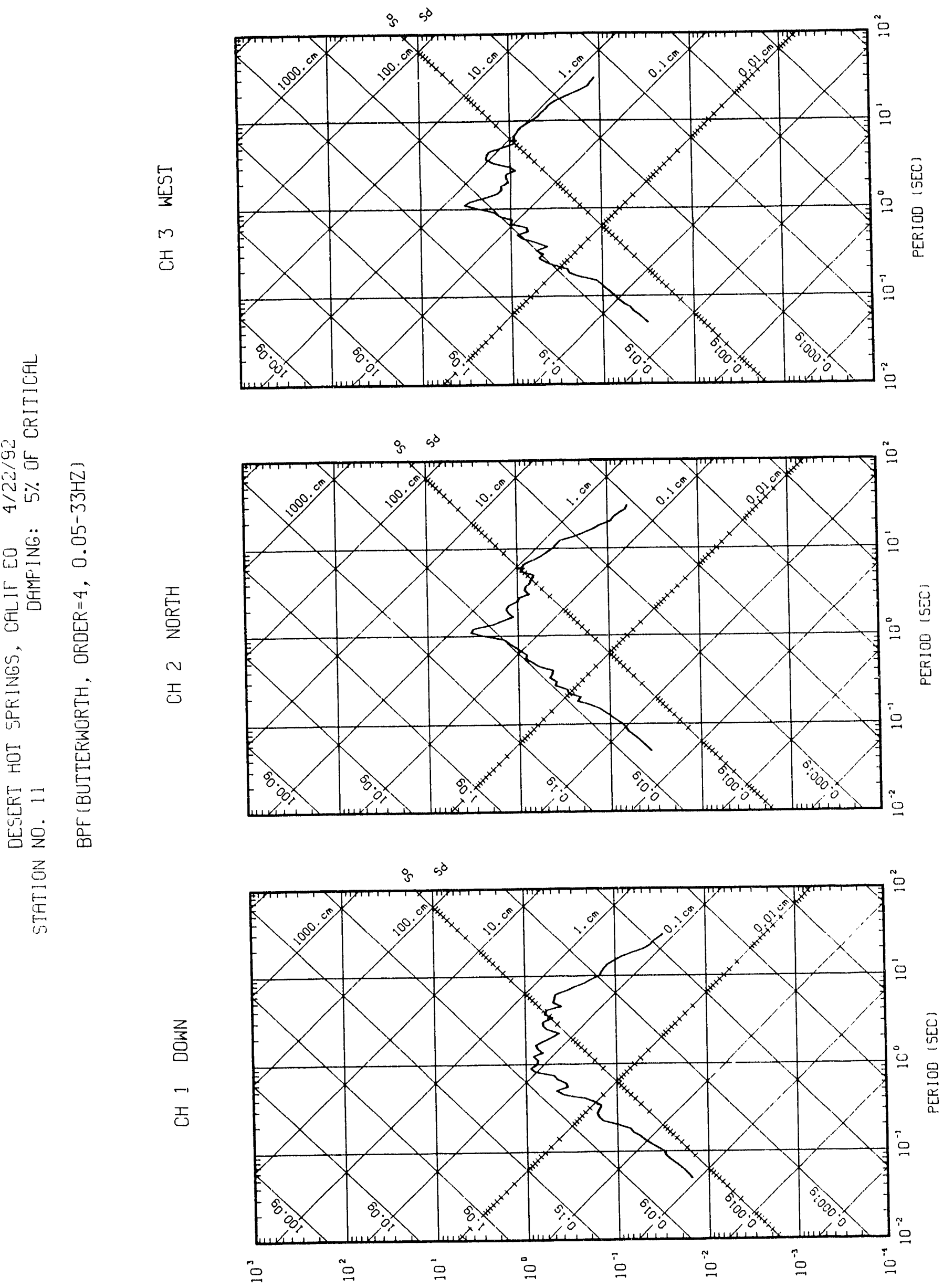

(J3S/WO) ^S 'KLIOOTJN JSNOdSJY OONGSA 
○

○

○

0

N

$r$

요

Z

$F$

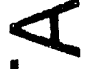

5

- 
$\frac{\mathscr{E}}{\frac{1}{3}}$
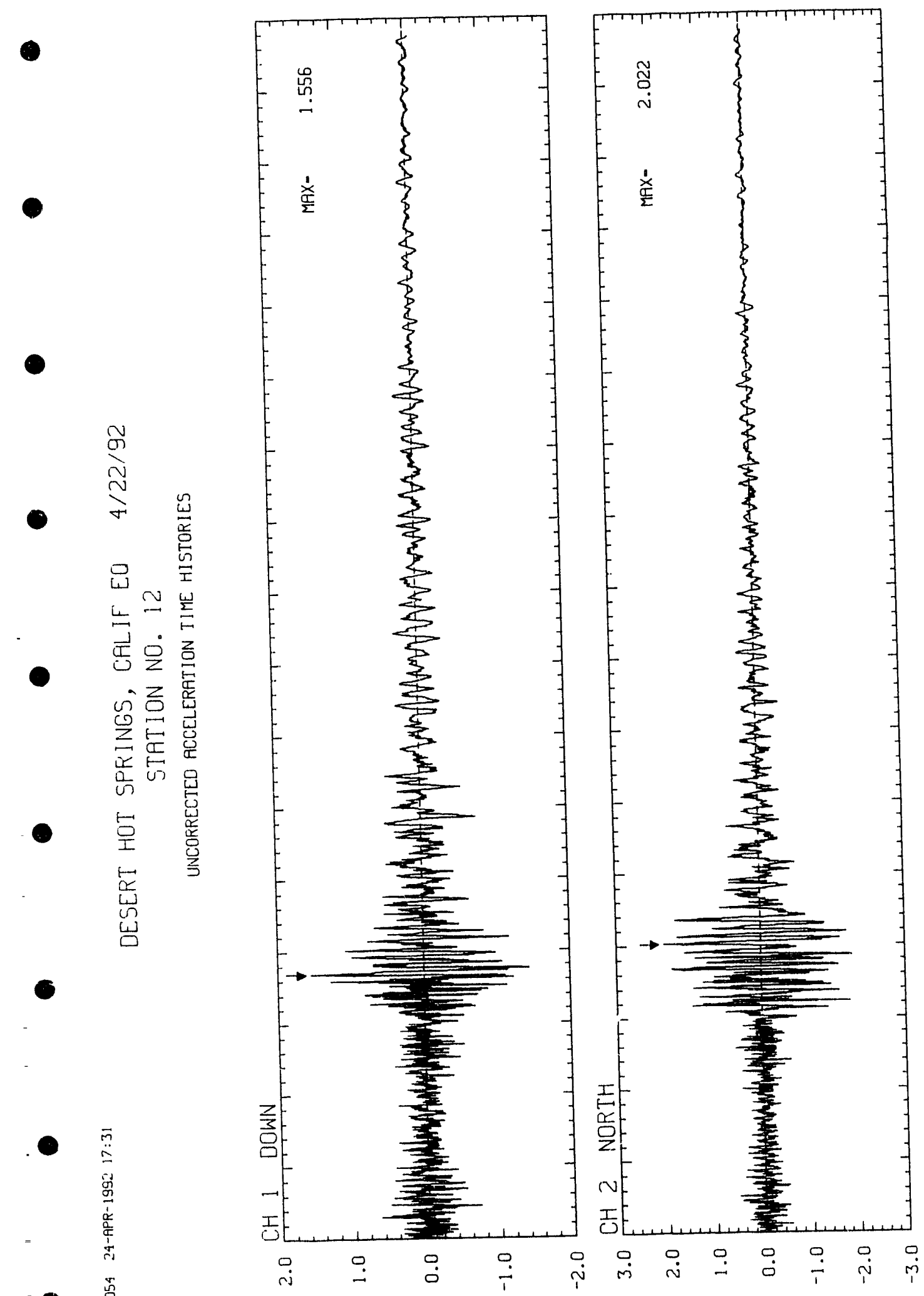

लै

怘

i.

늘

䨔名总

i呑

怘

品点

- 占

占岕

$\breve{c}$
$\underline{\omega}$
$\omega$
$\omega$

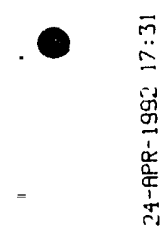

骂

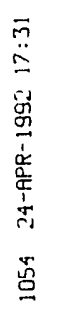



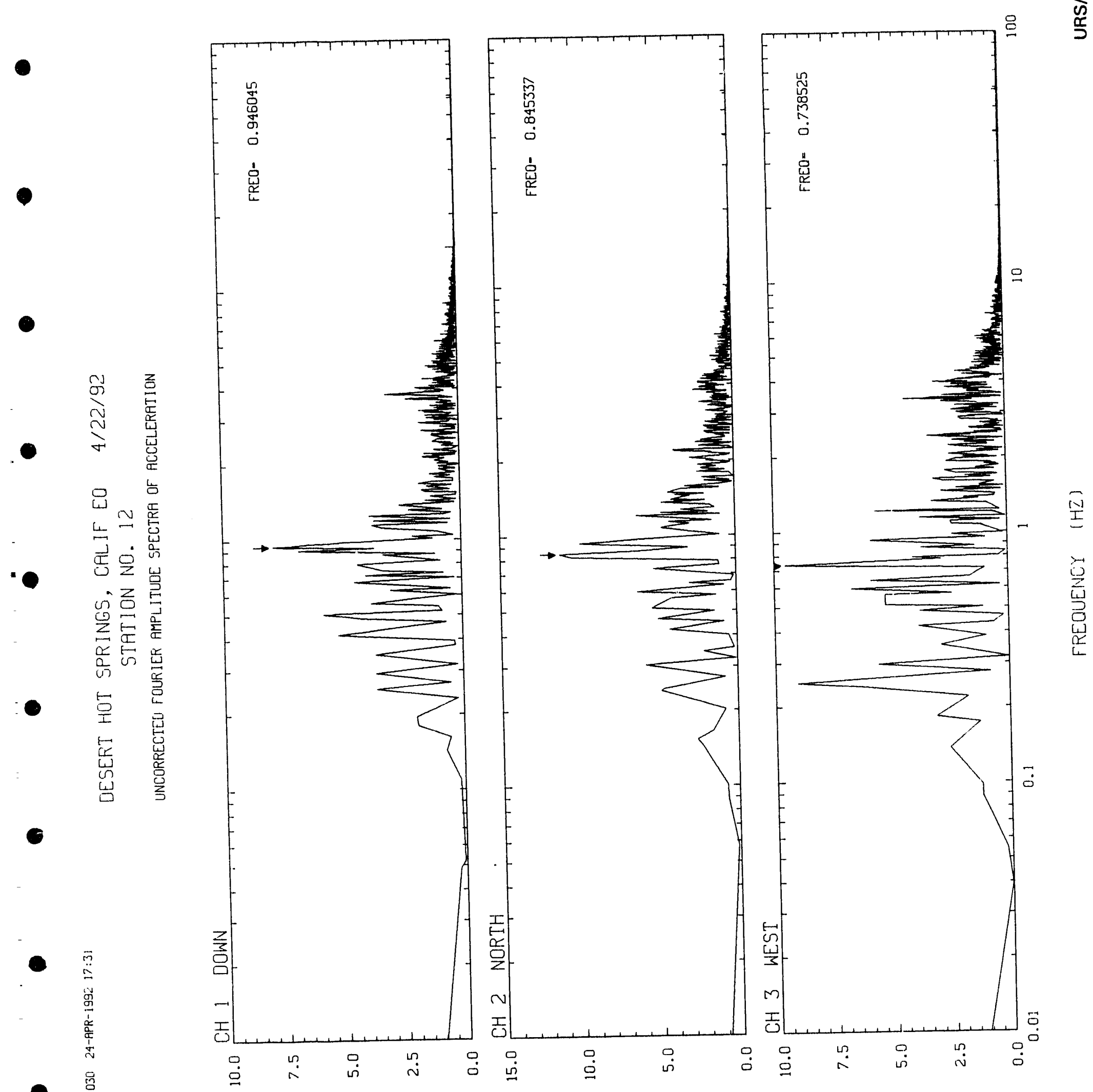


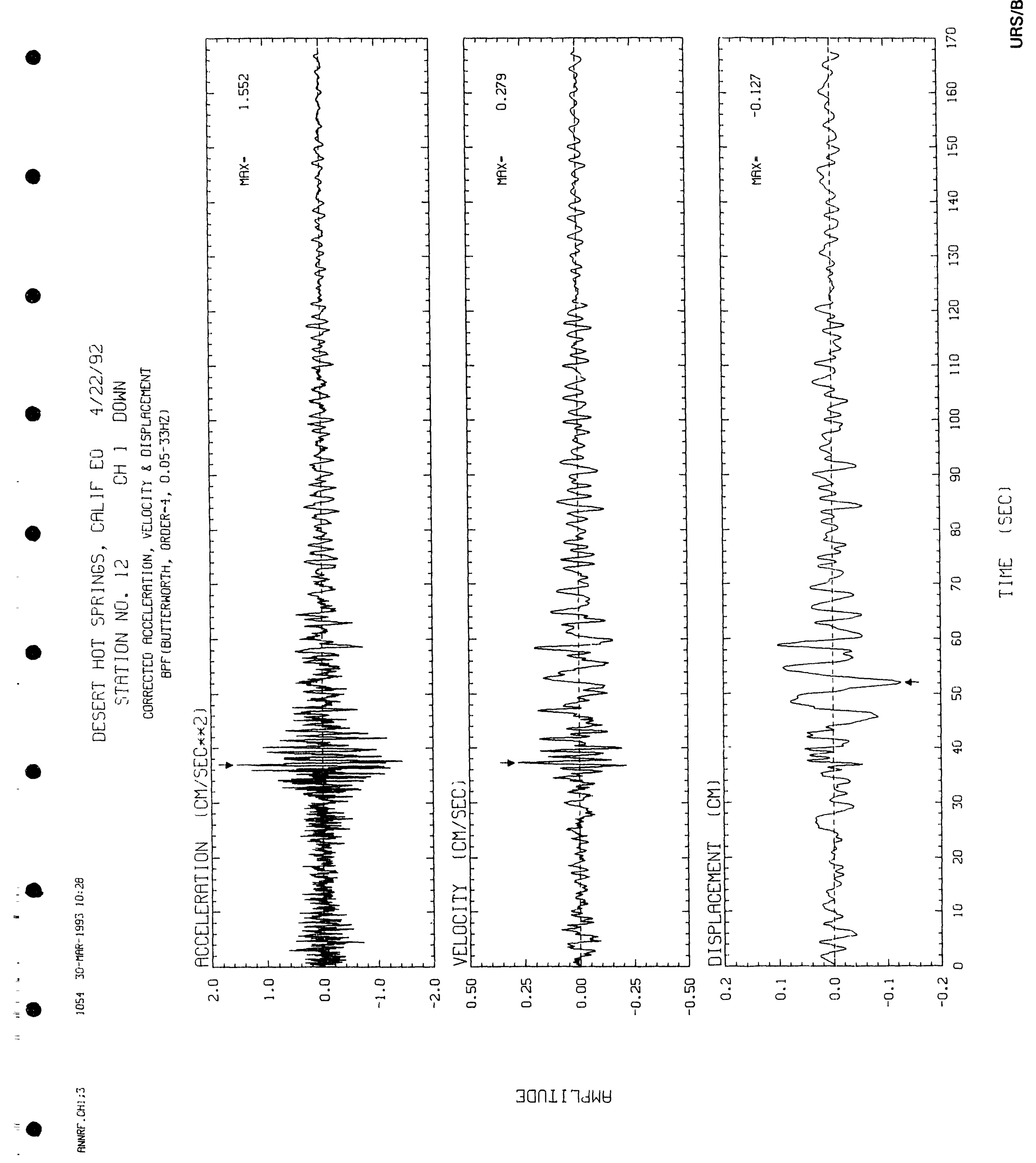




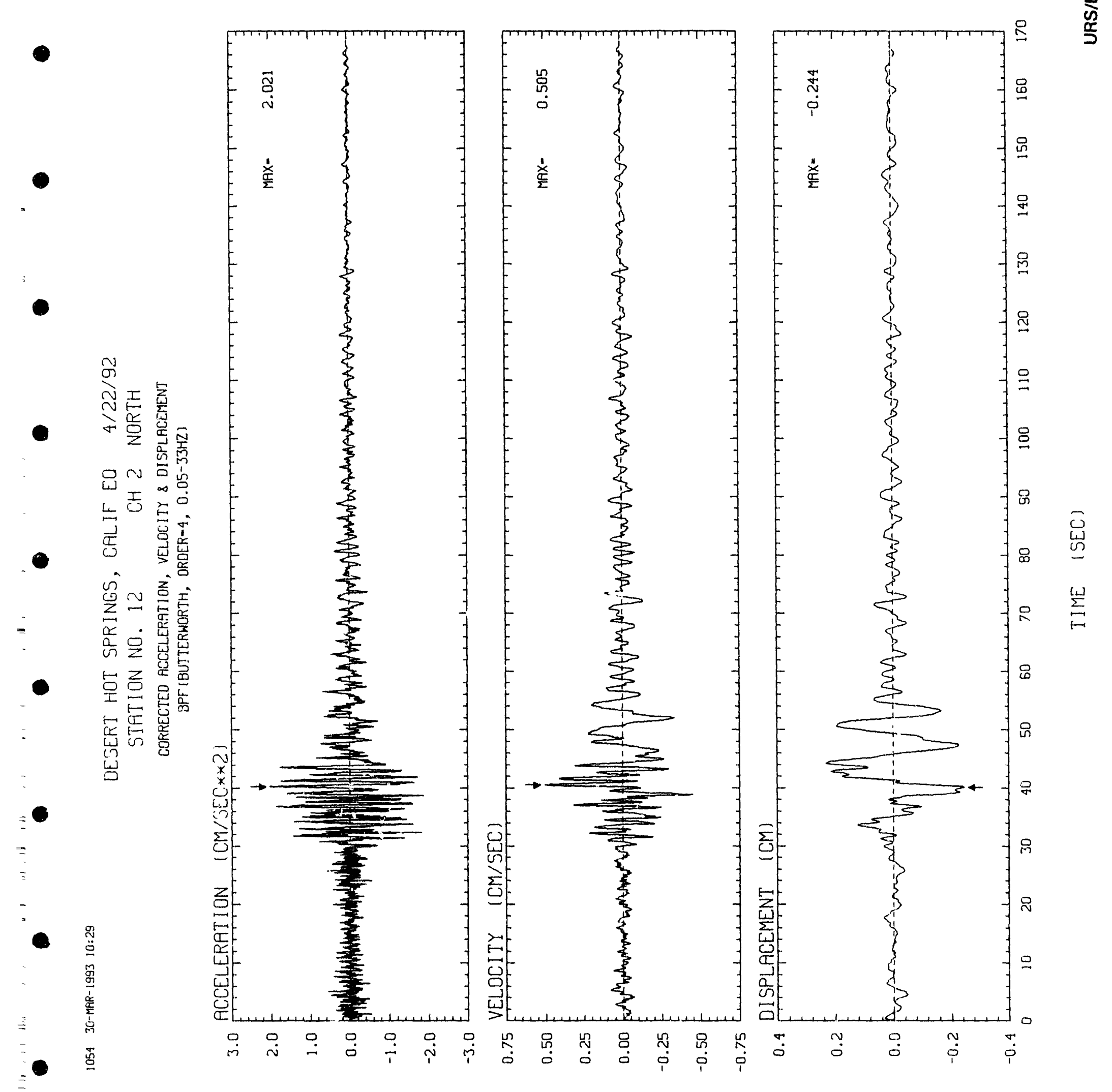



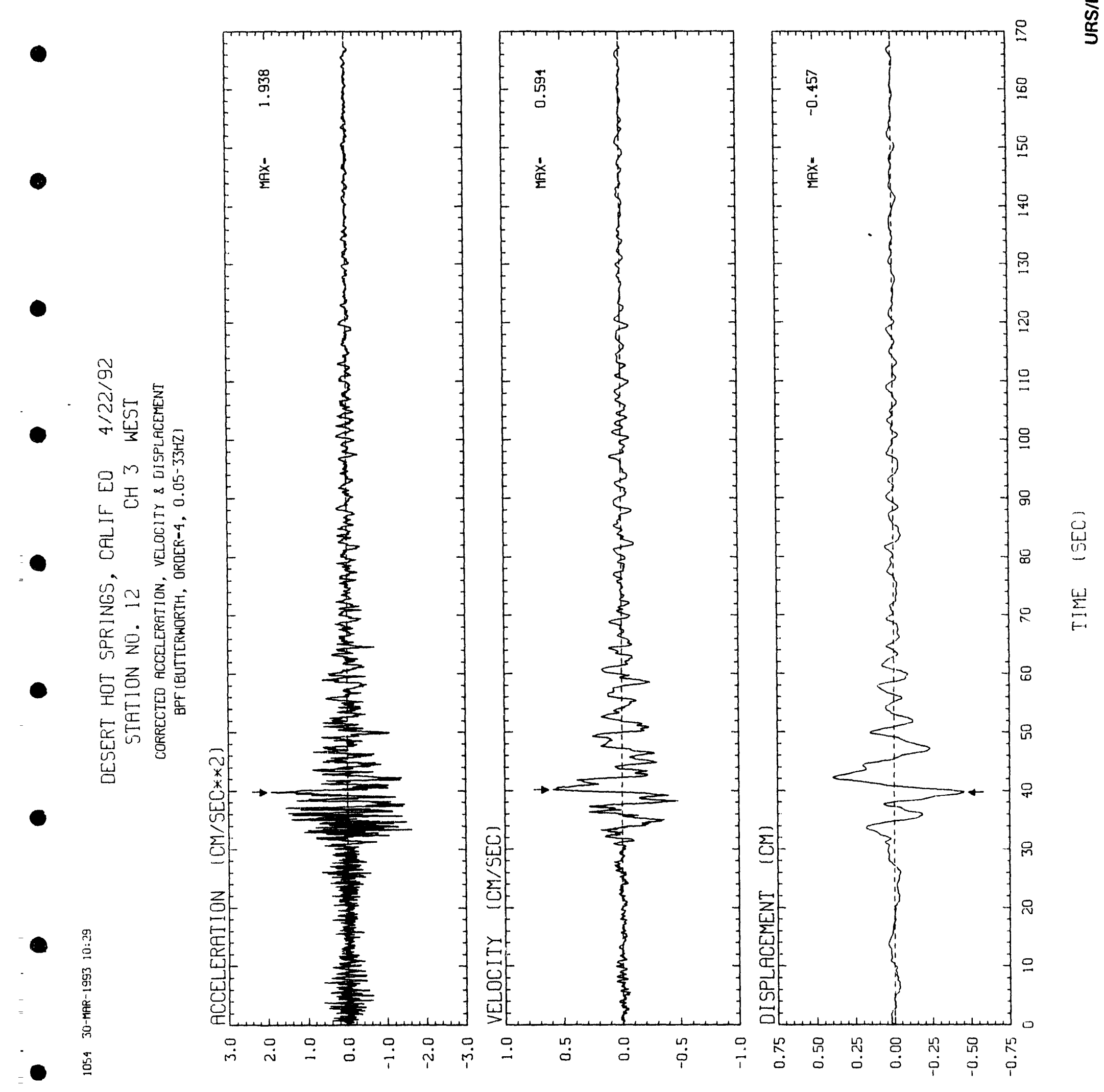


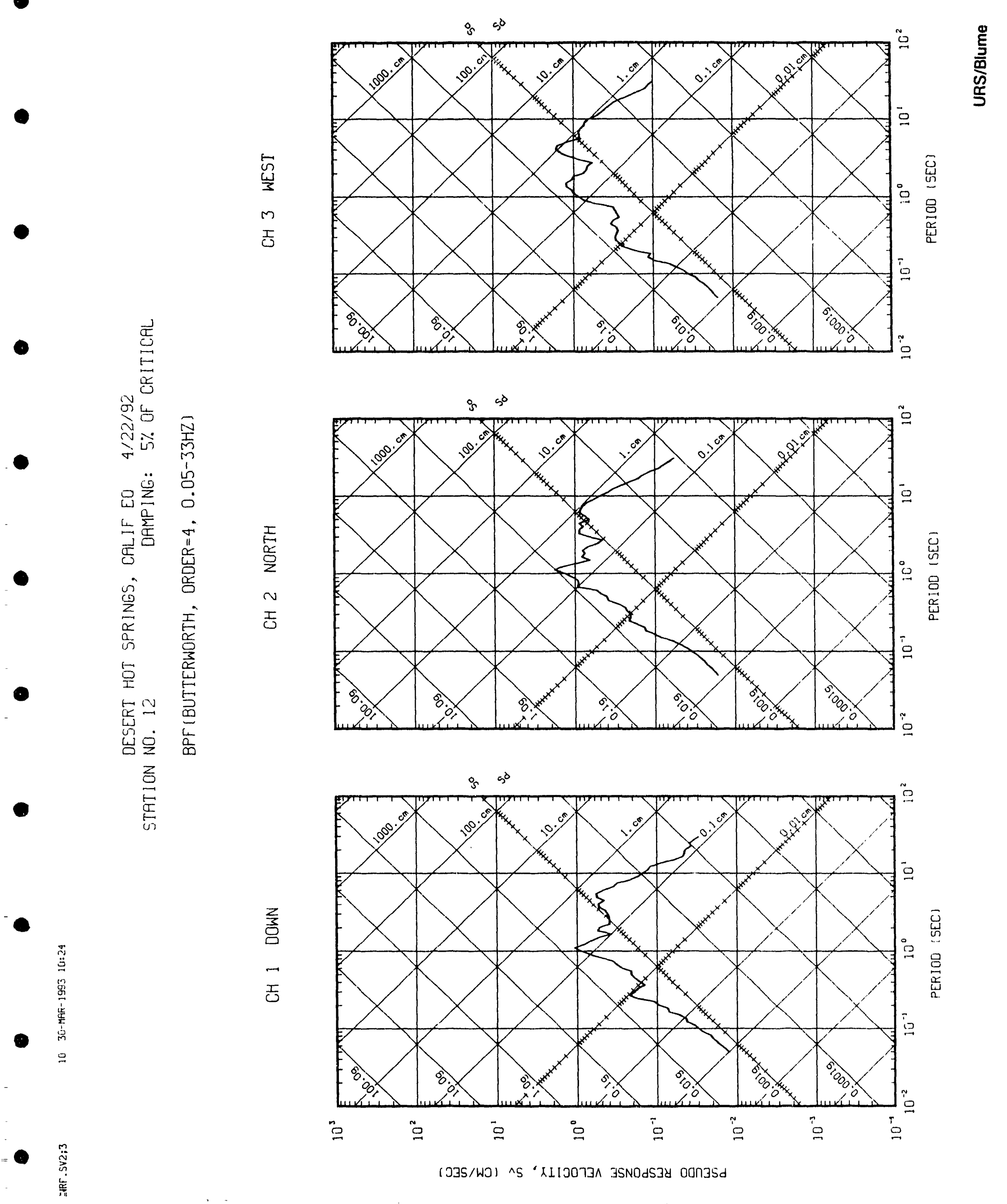


@

(1)

Z

E

ज 

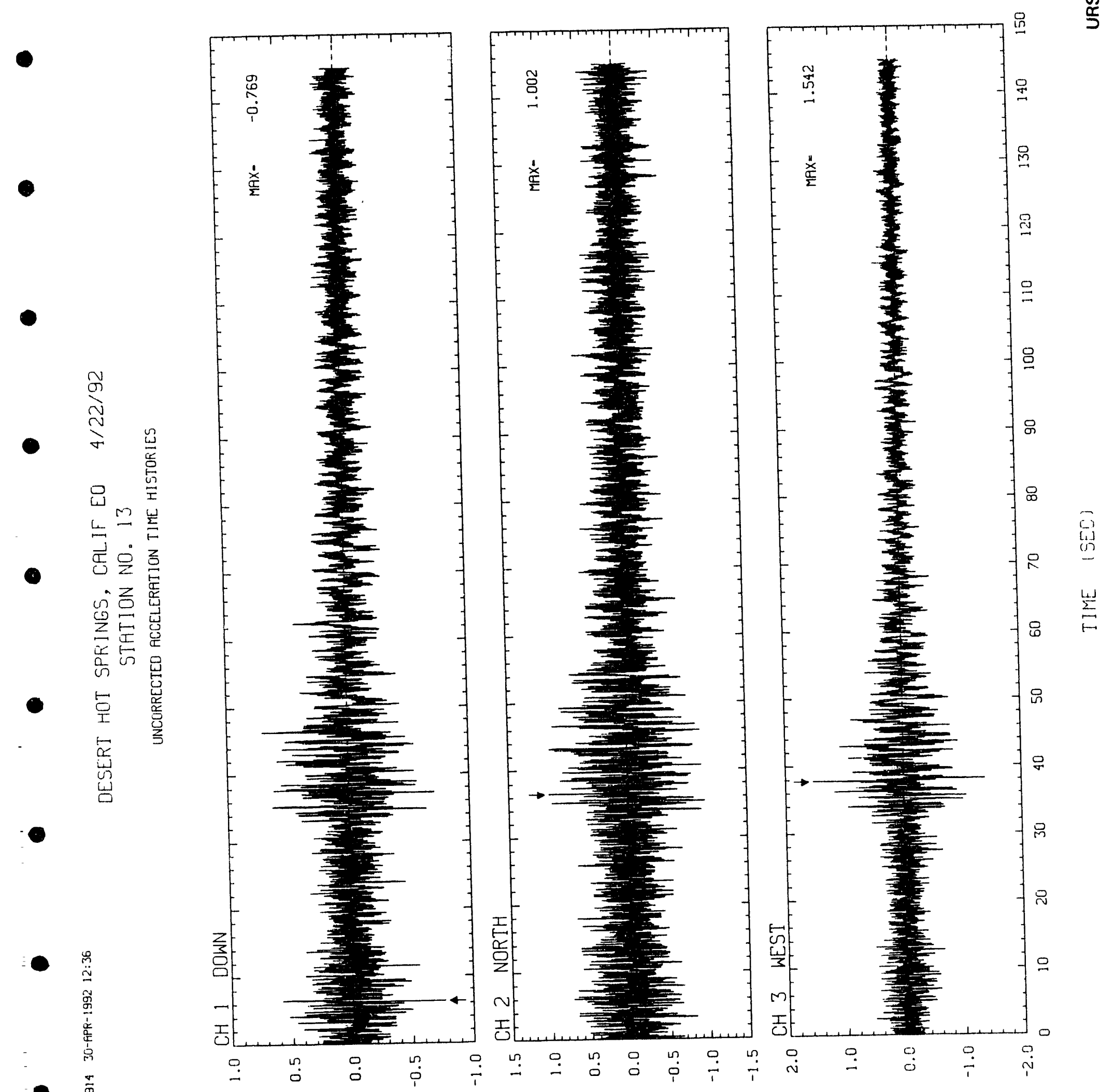

(2**כ35/WO) NOI I $4437 \exists J J 4$ 


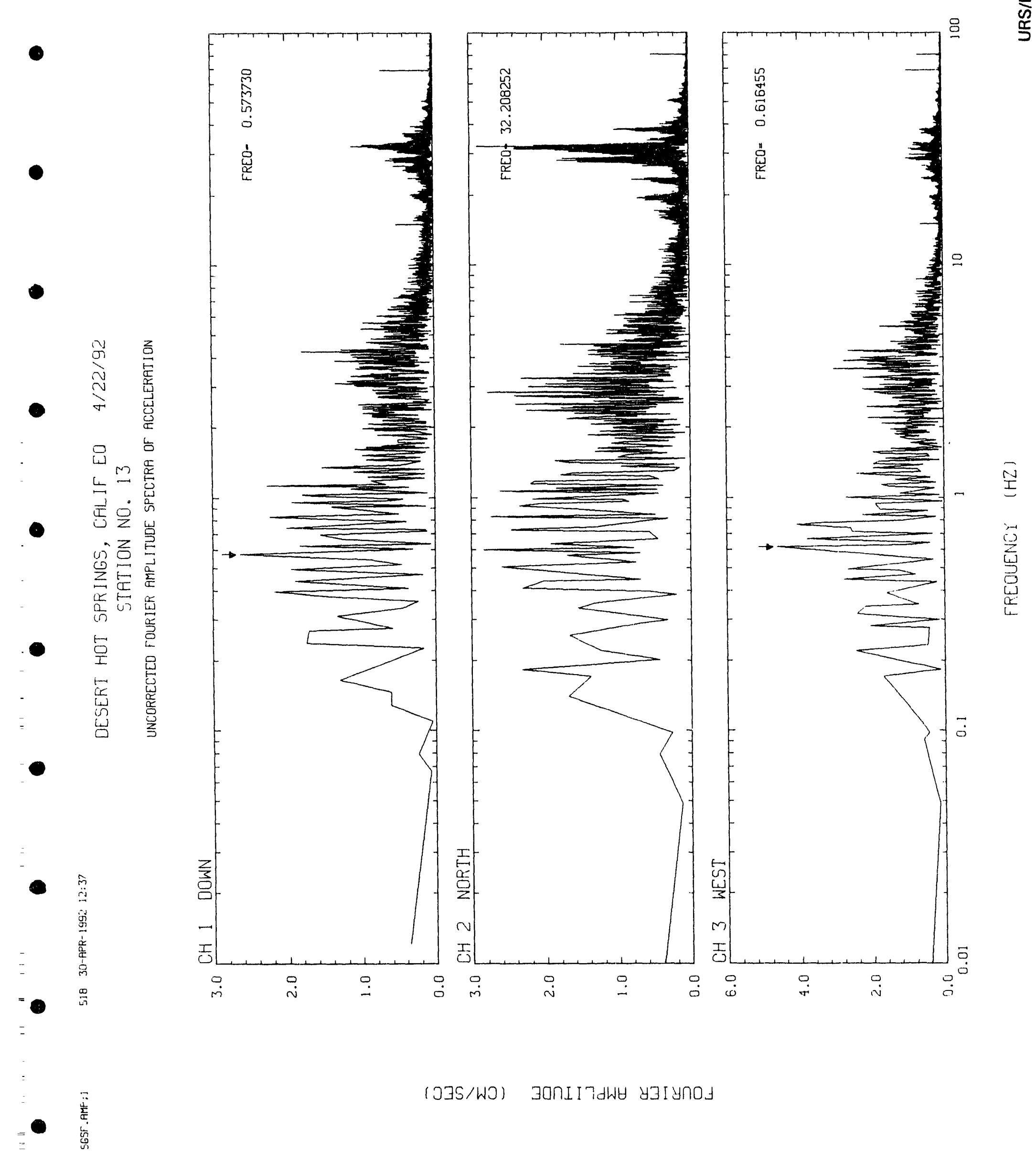



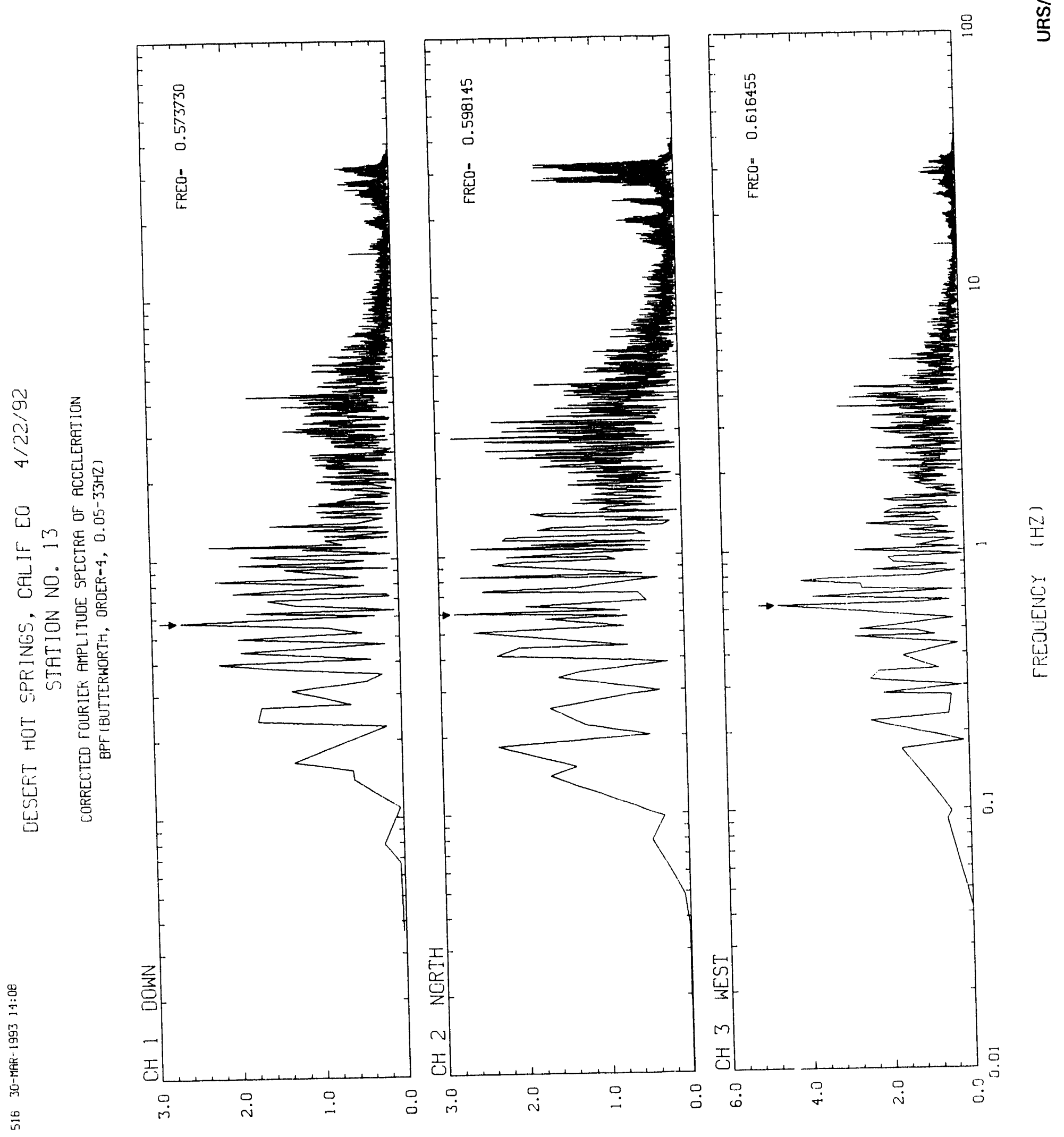

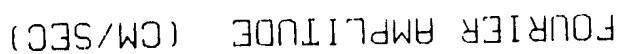




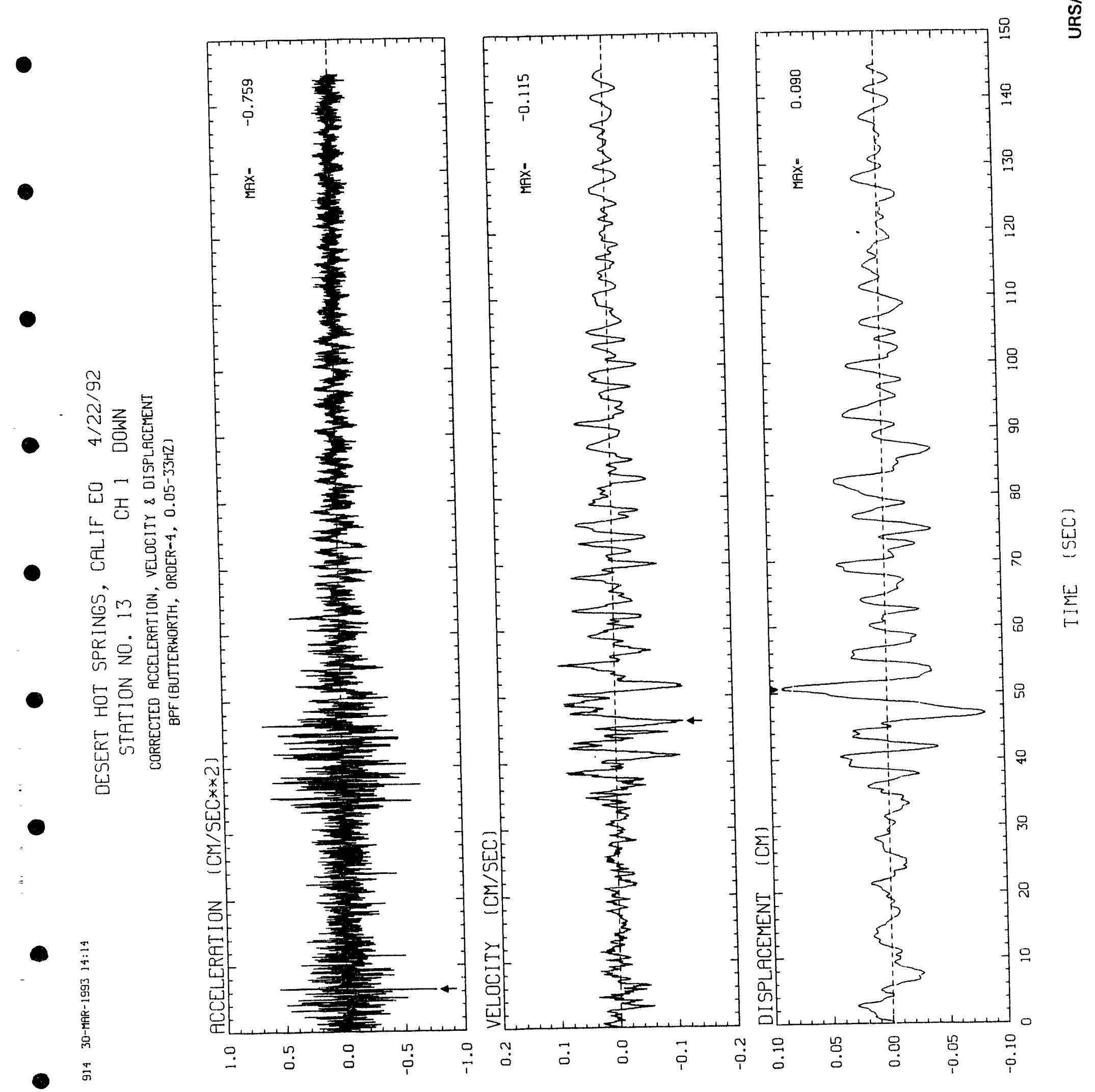




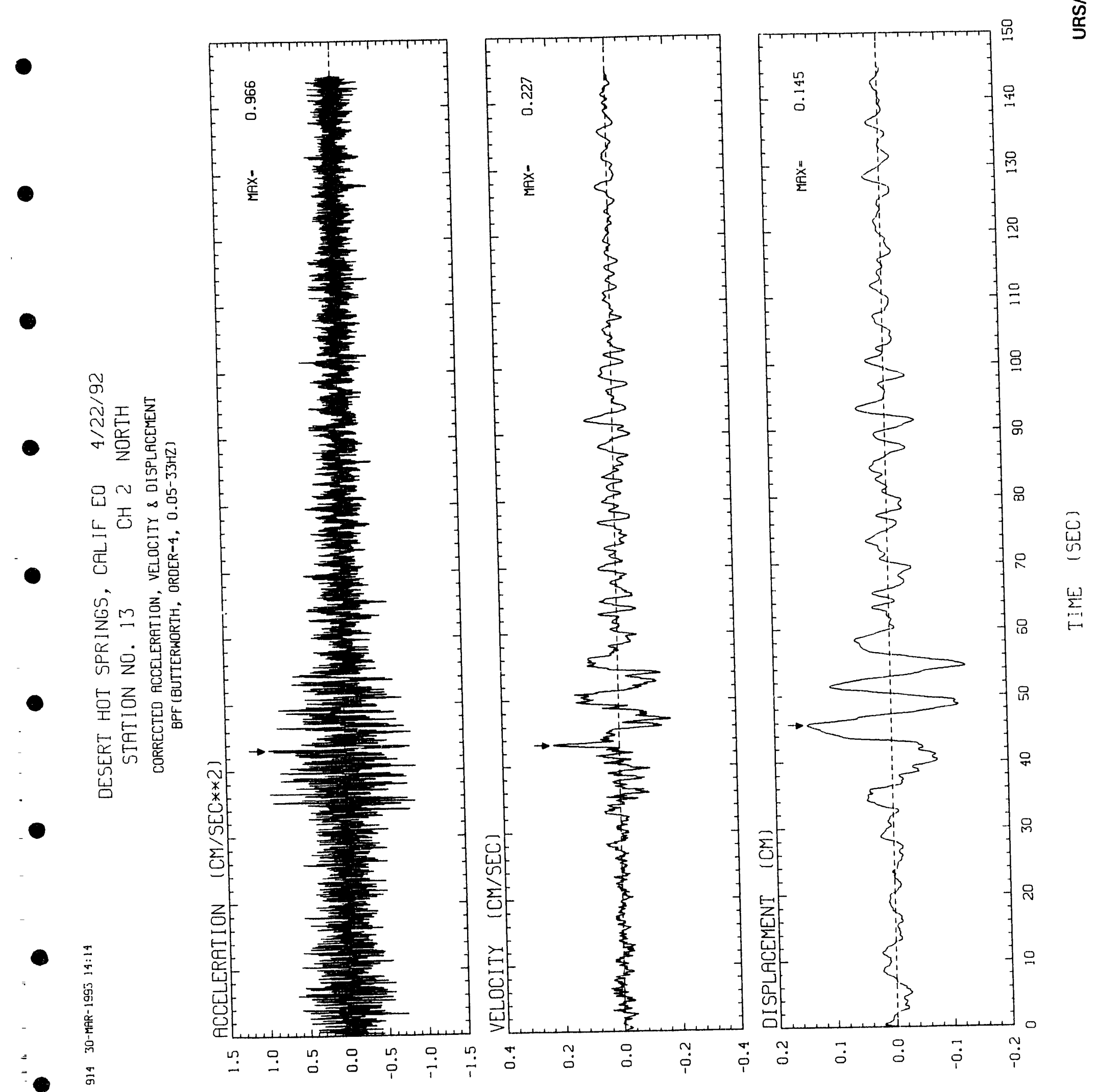



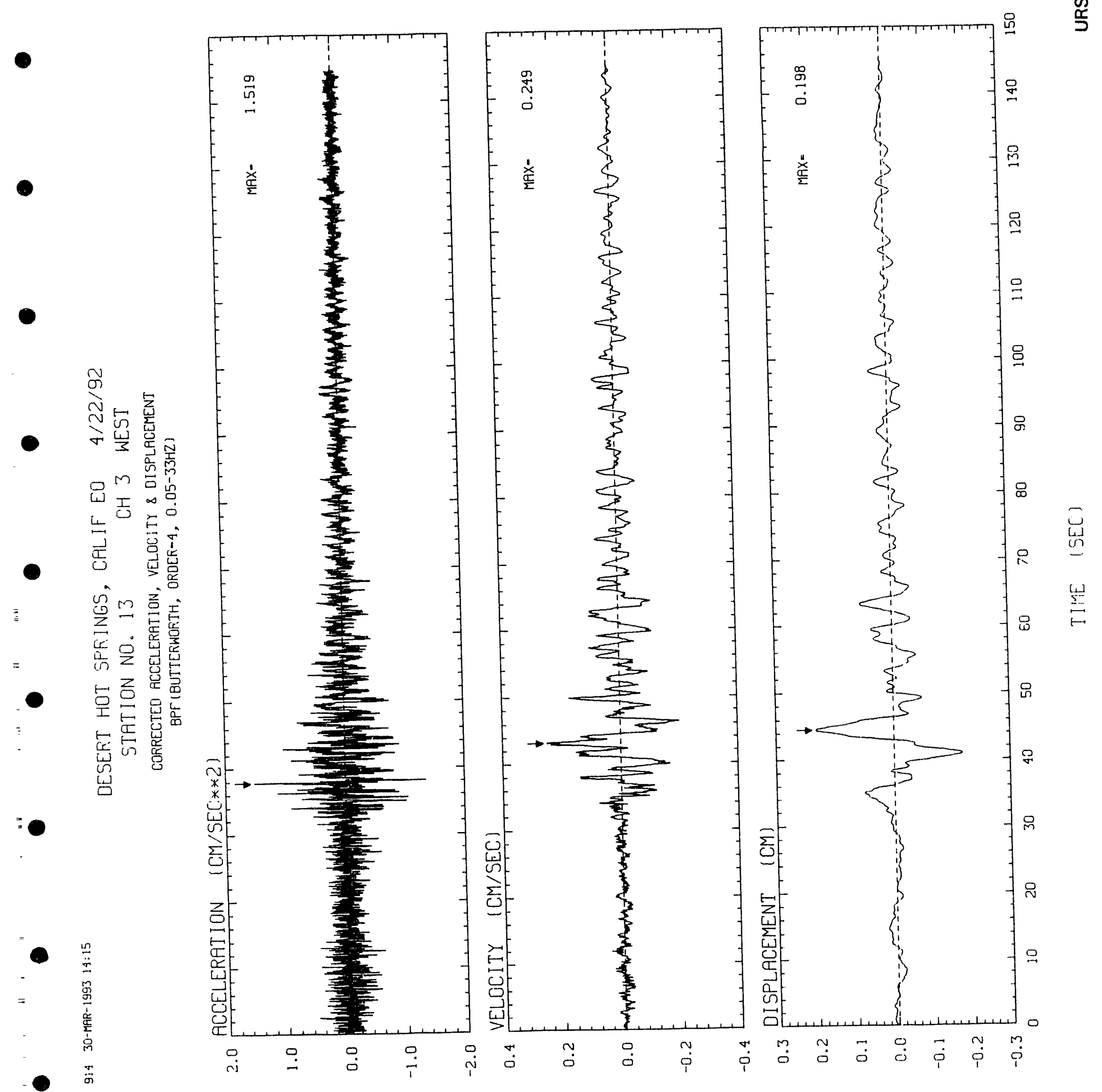

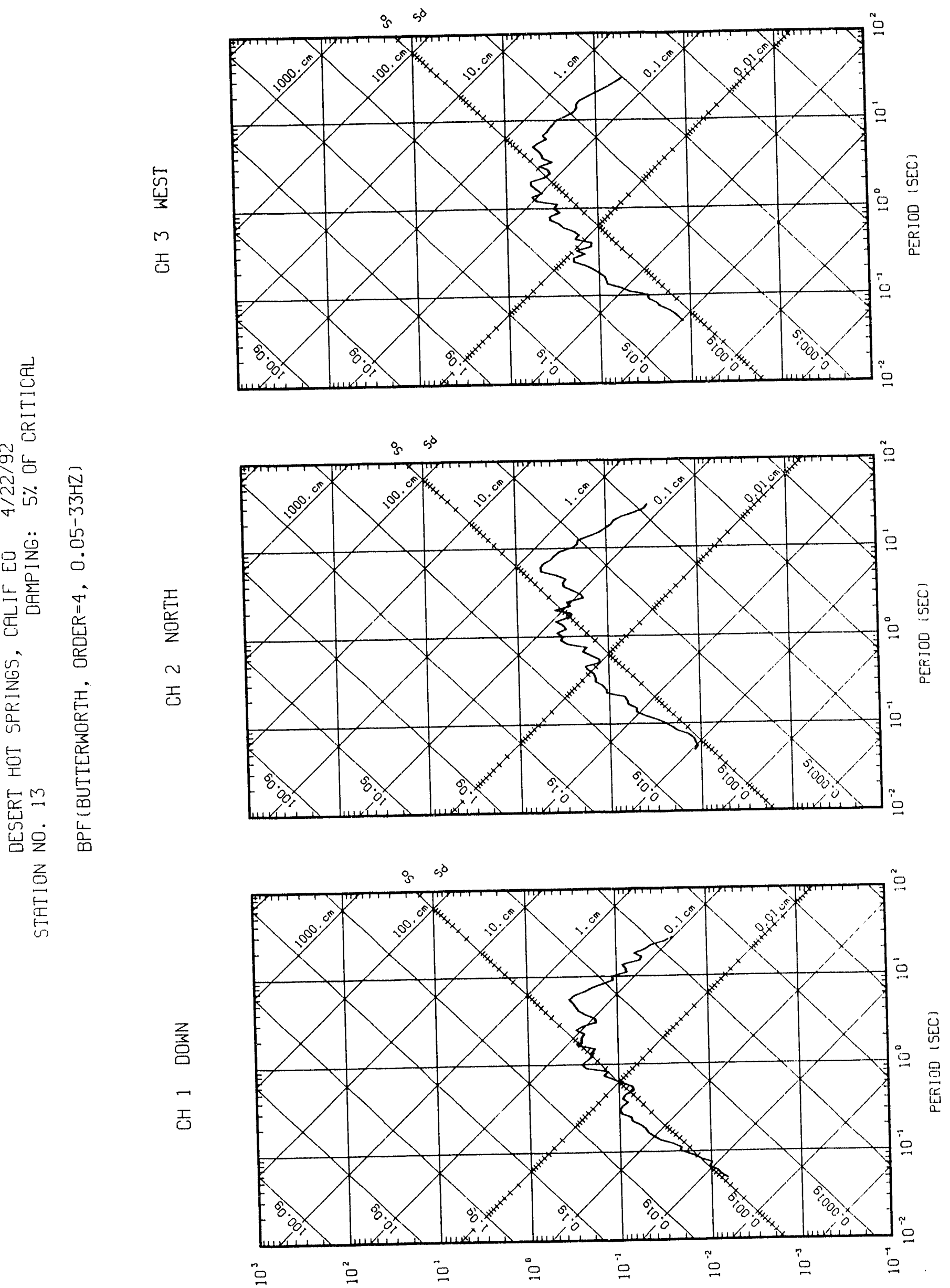
$\bullet$

癹

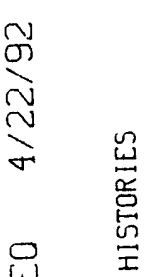

岂地

死完啹

证琶

怘豆

品帛品

- 萣

点

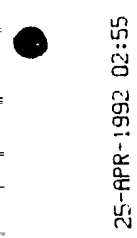

-
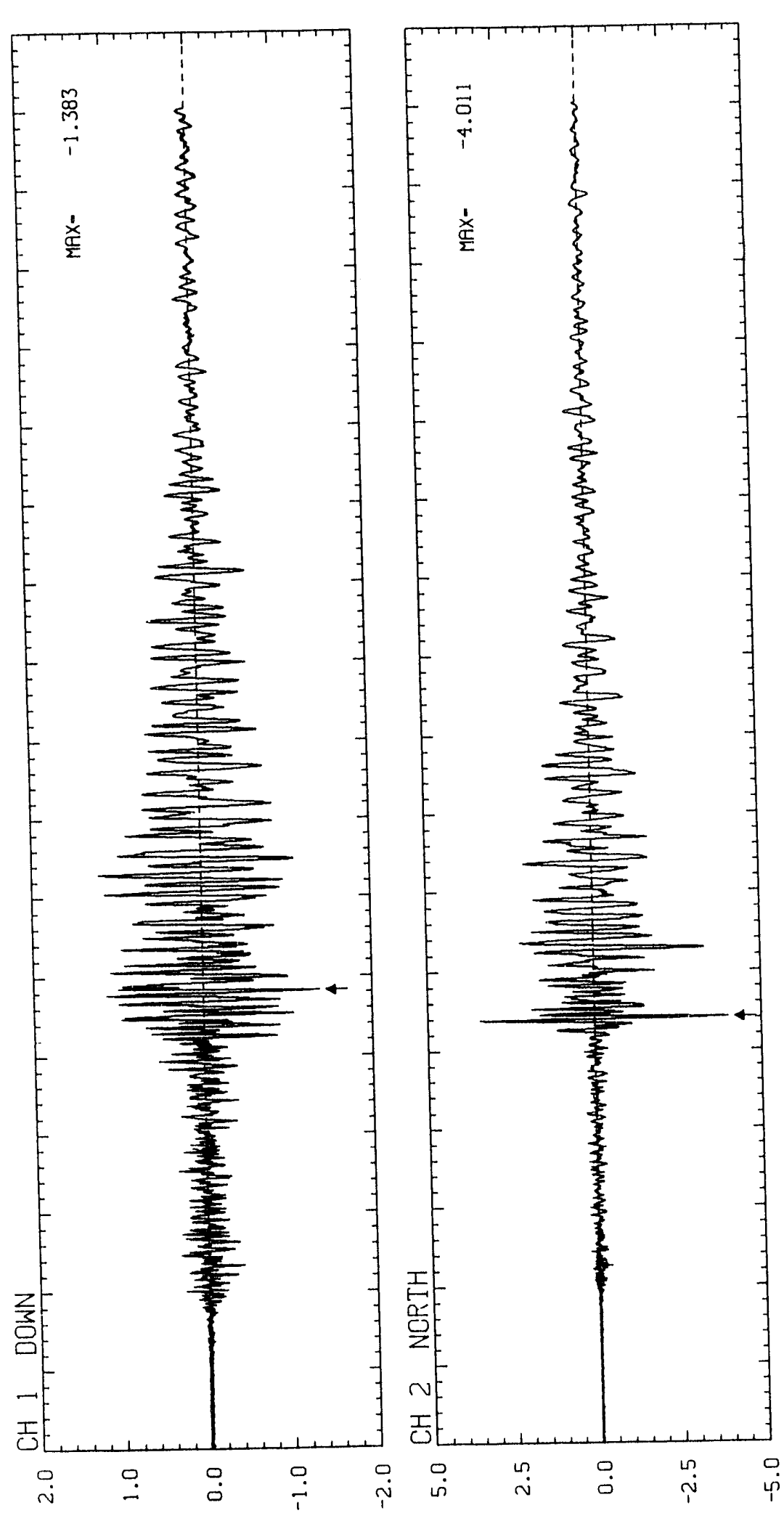

$\frac{\pi}{2}$
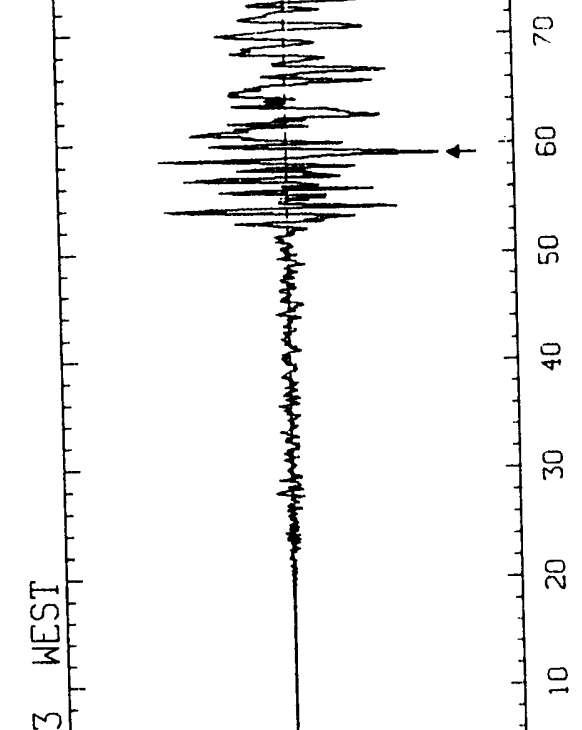

돈

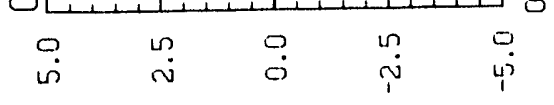

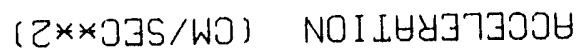




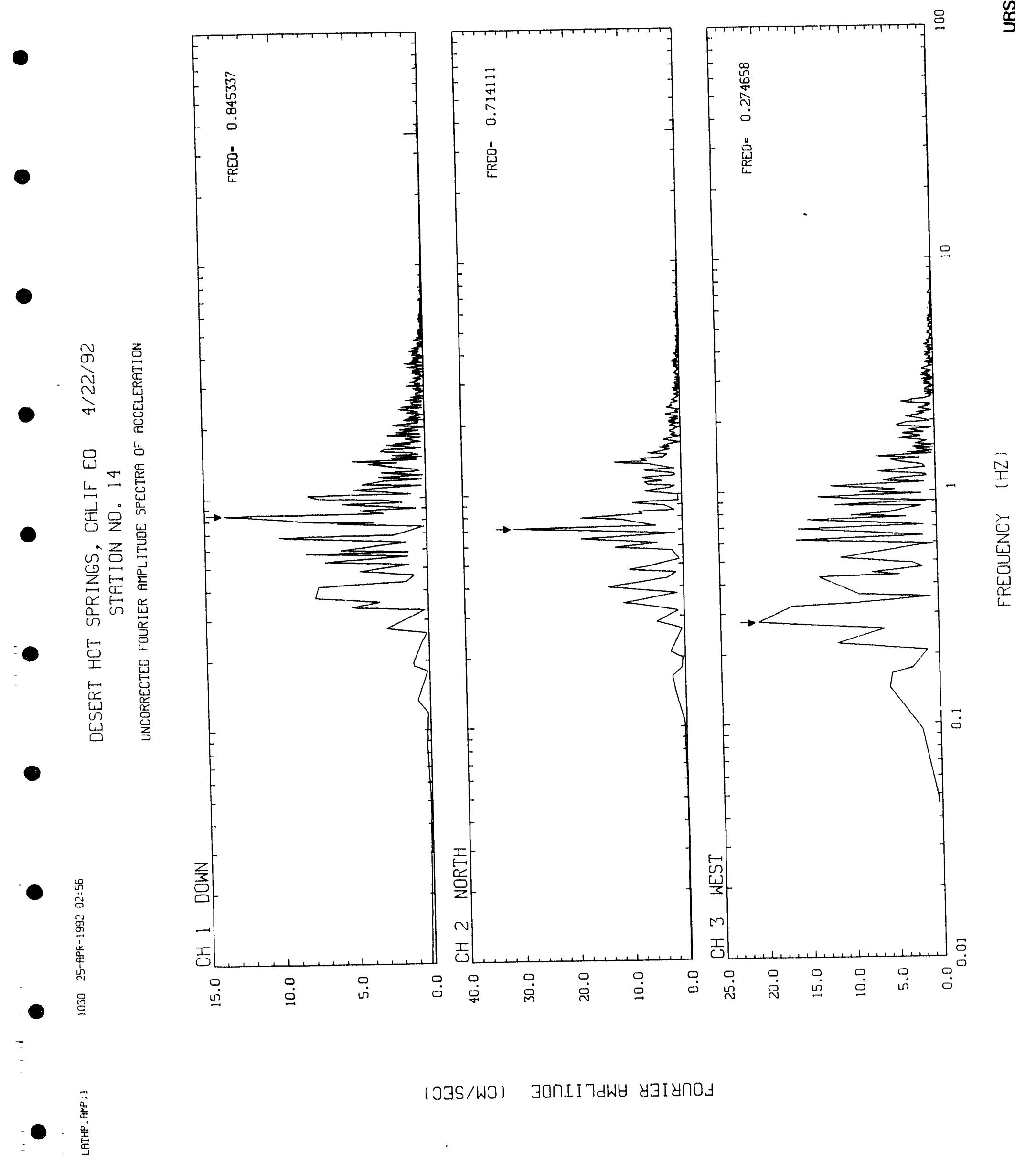




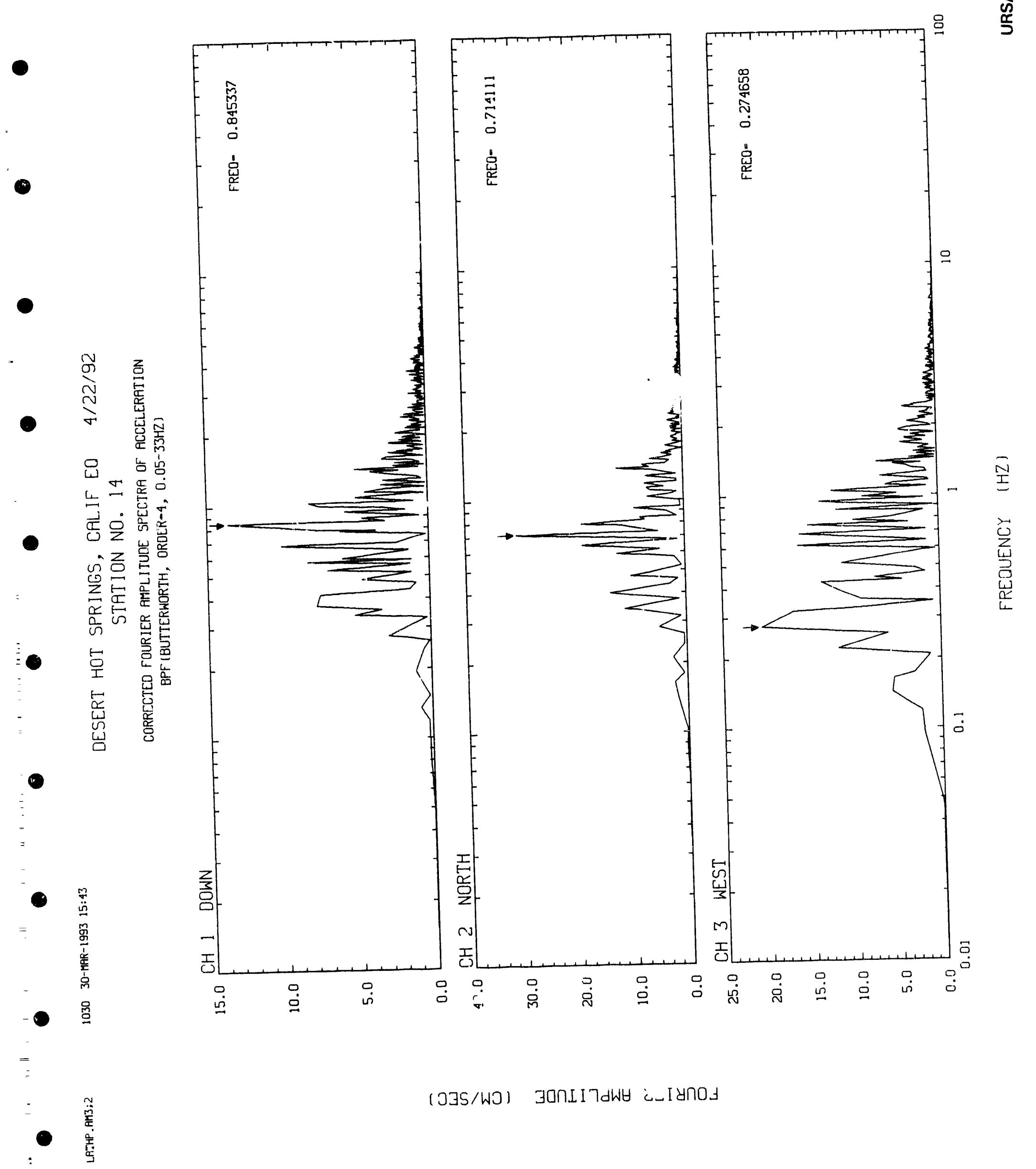



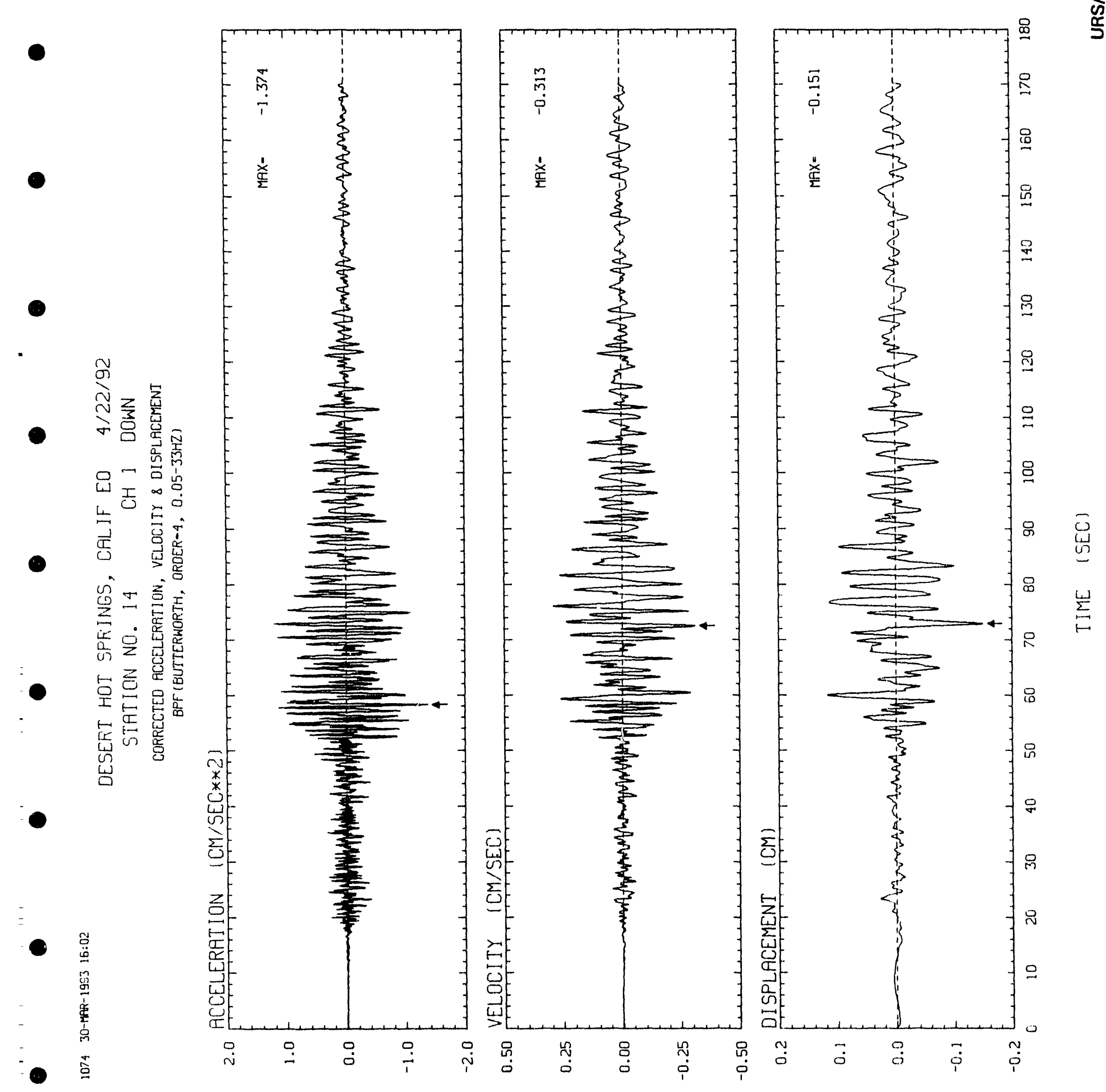

30ก1 I divity

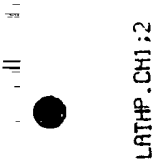





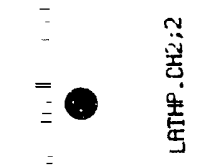




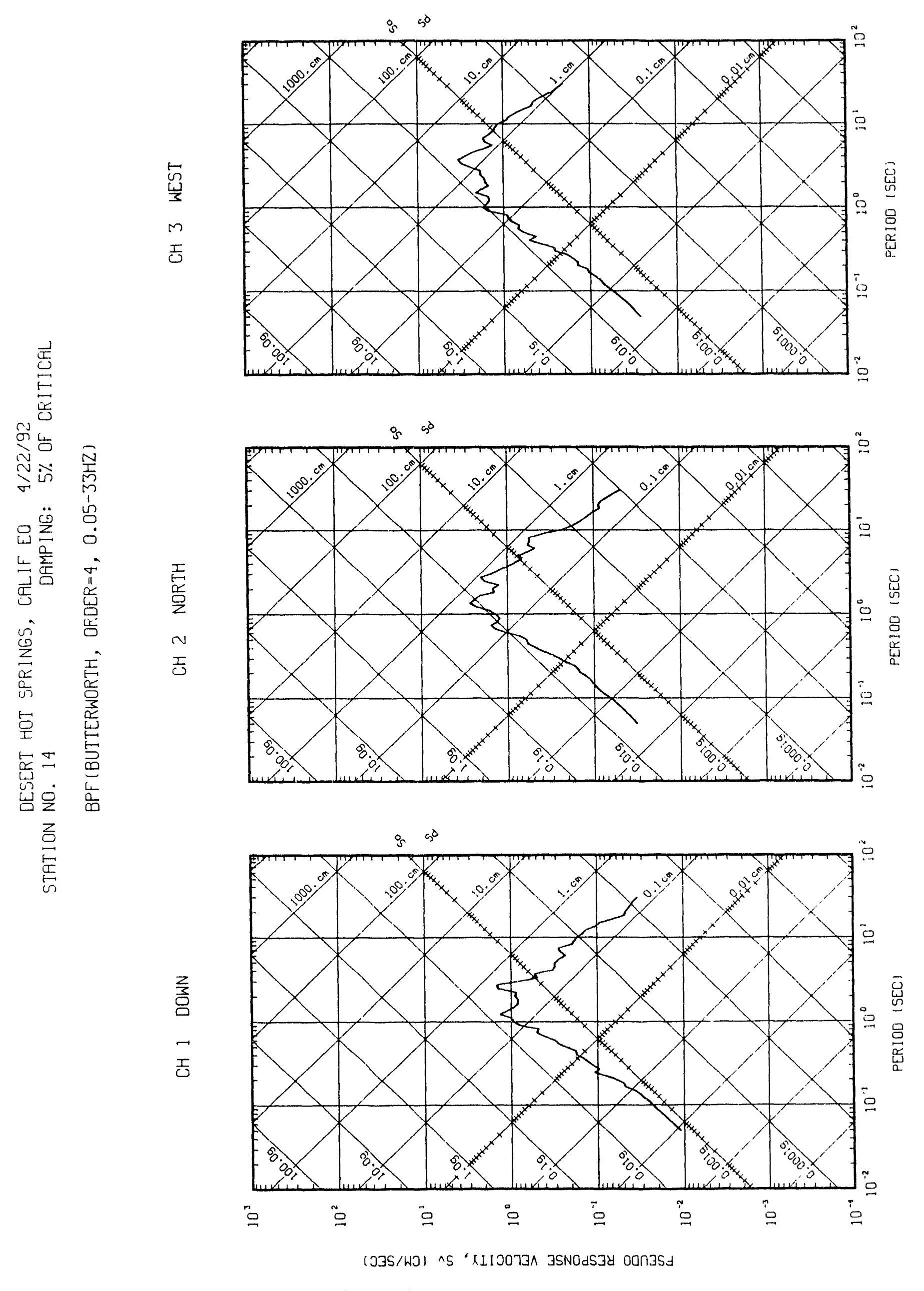


$C$ 

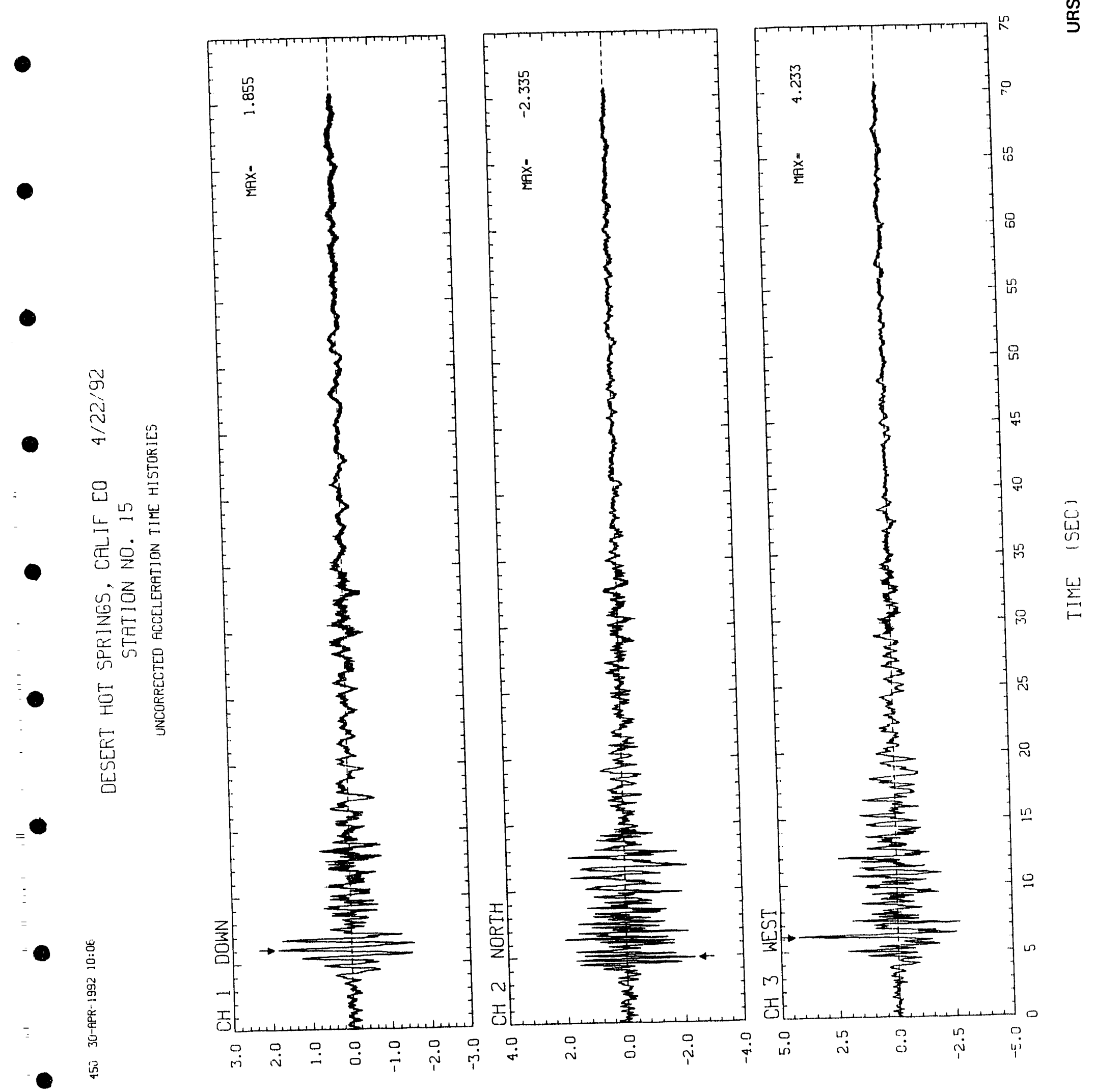

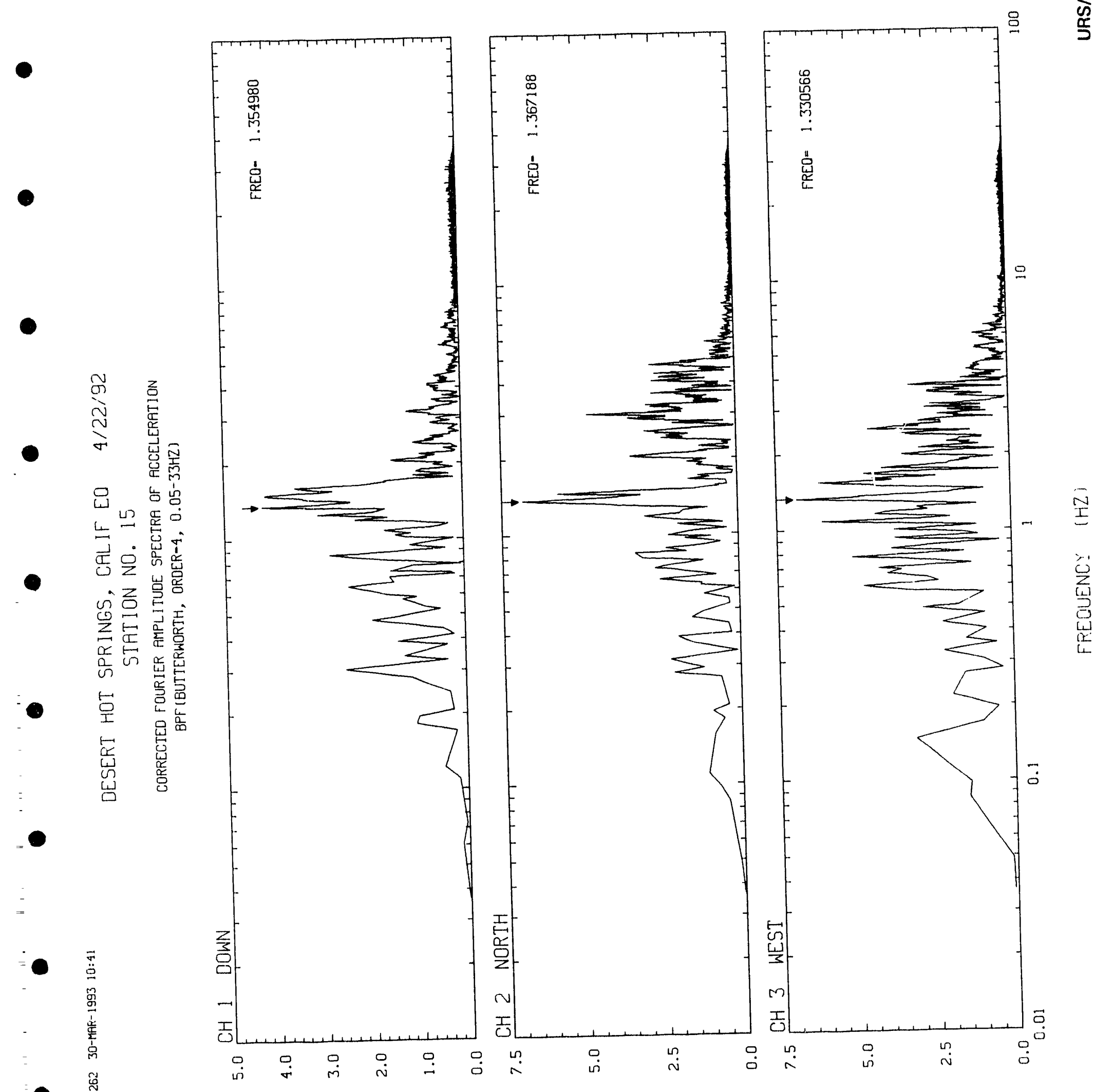


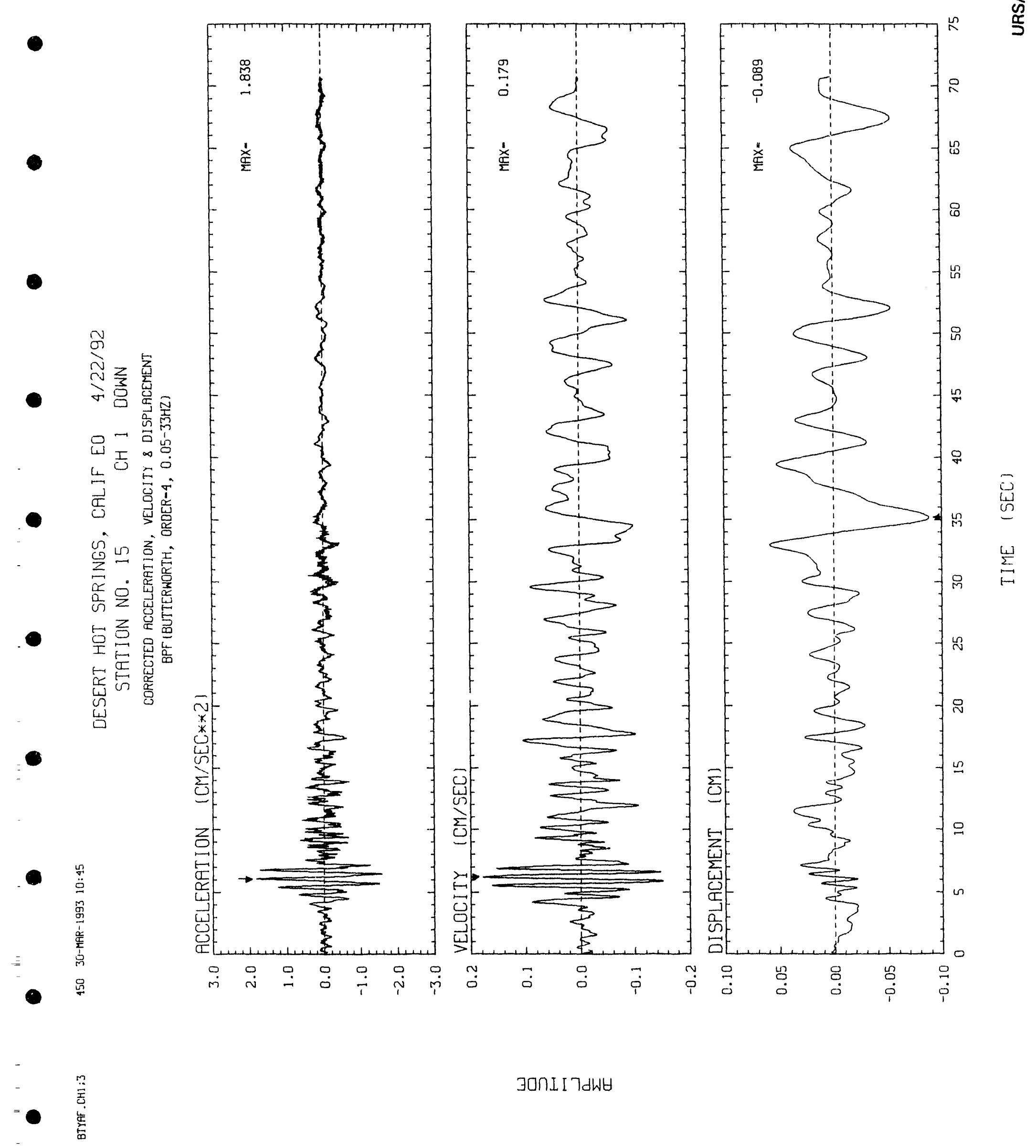




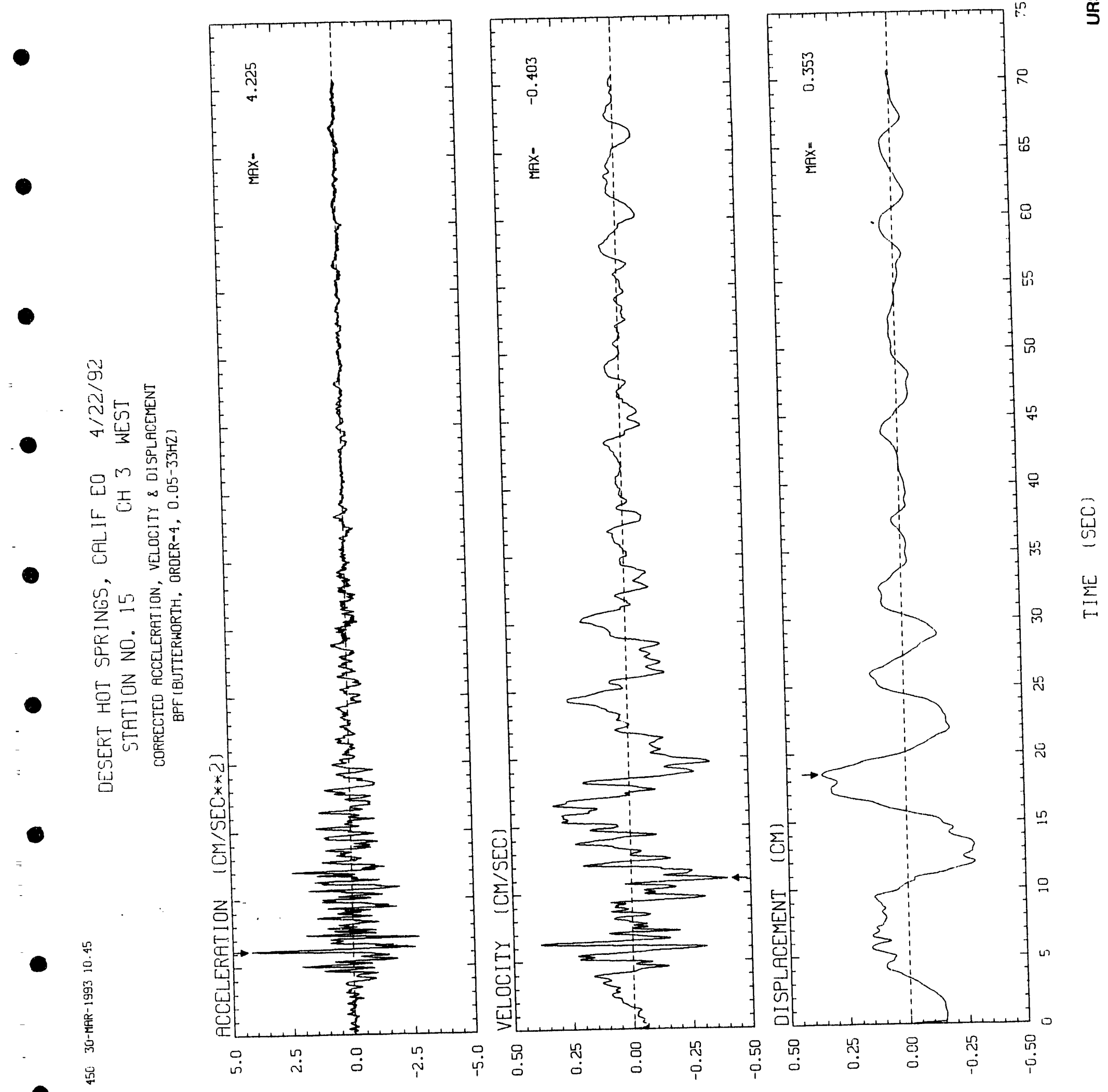




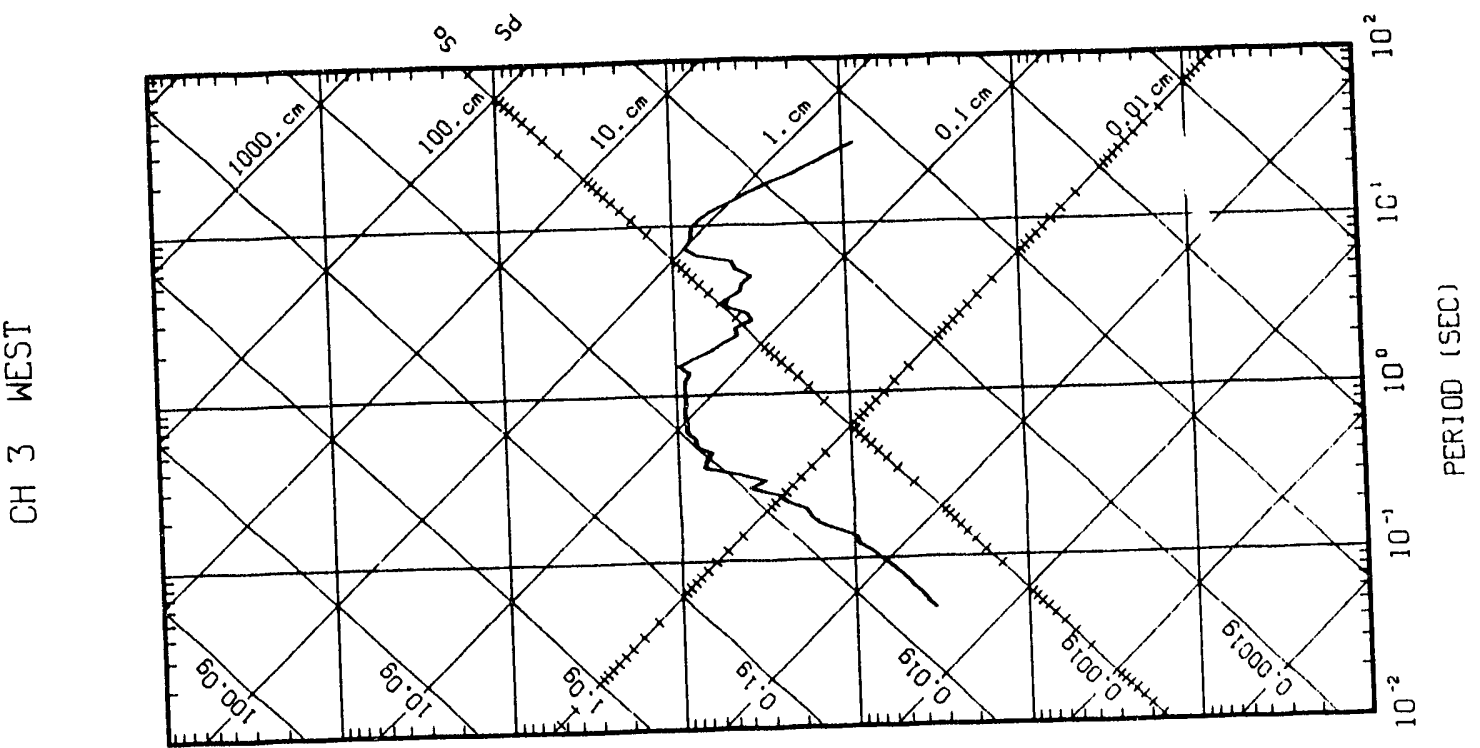

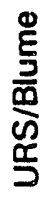

覀

बั

तु

㞻苍吕

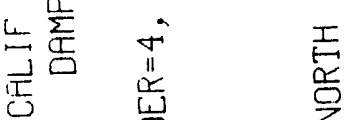

$\therefore$ 足

$\stackrel{0}{2}$

름

o

五

它

峲. 㟔呆

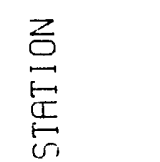

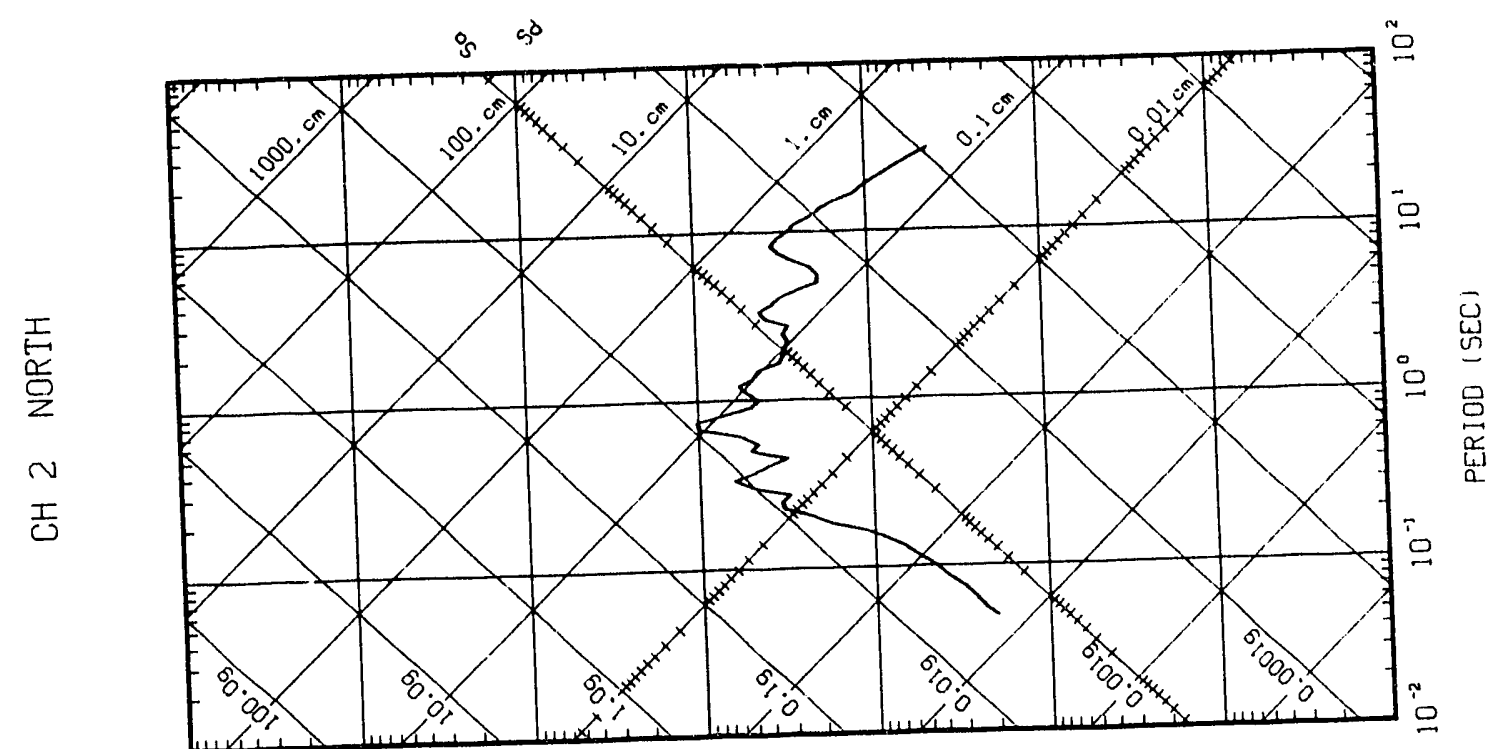

蒠

$\equiv$

弯

交

응

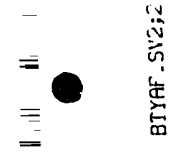

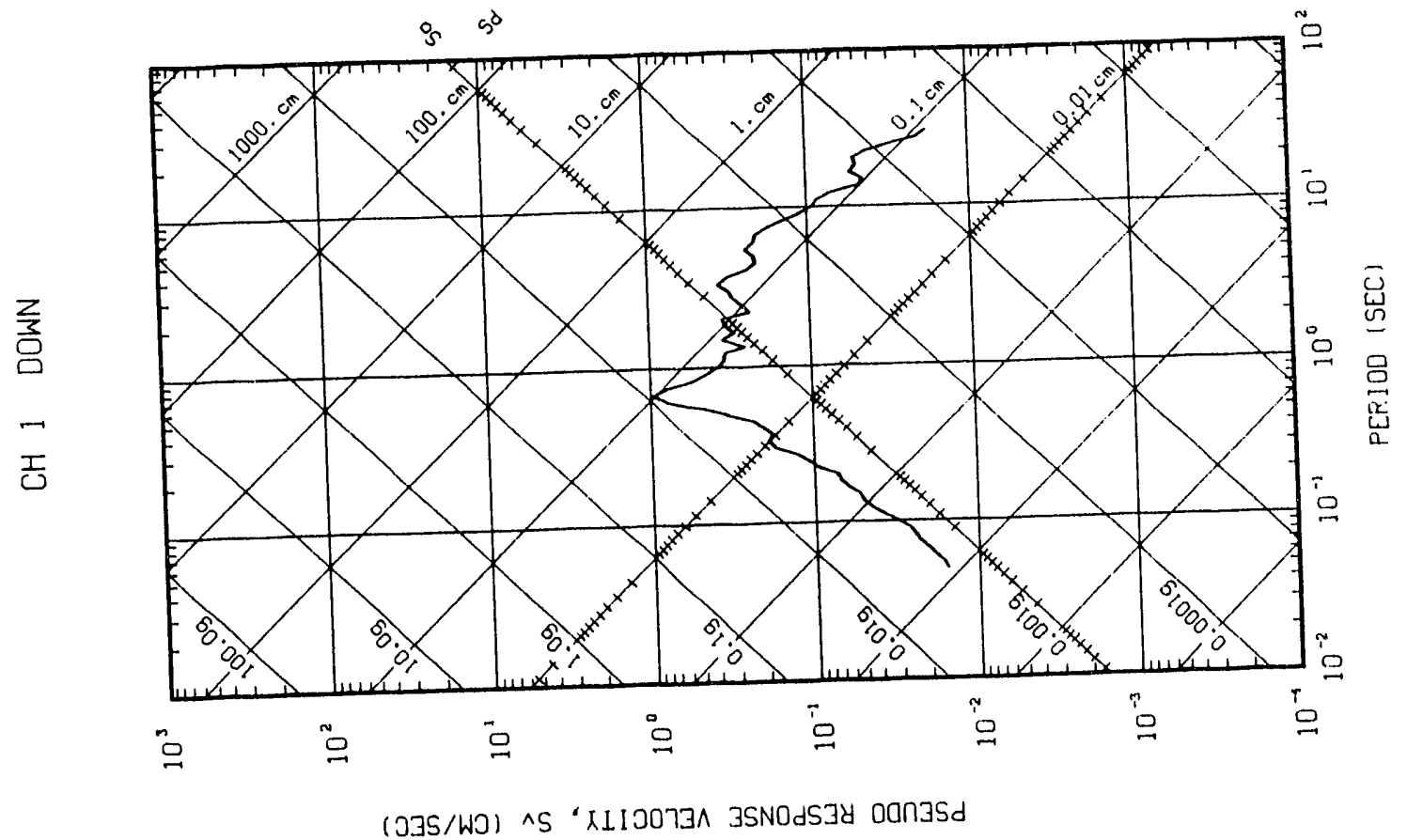


6

$r$

을

$z$

O

E

5 

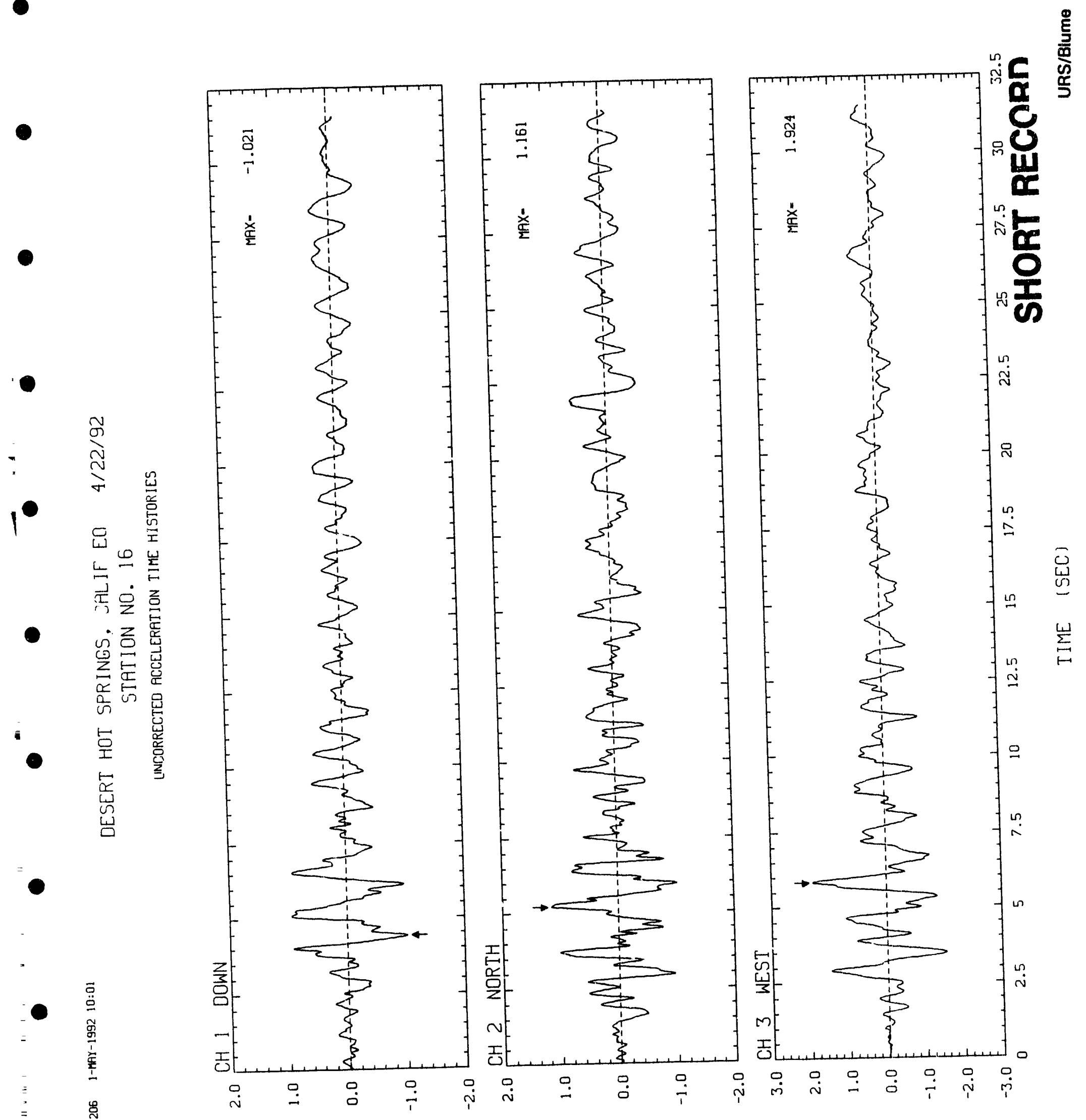

(2**J3S/WJ) NOI It'y373วJH 


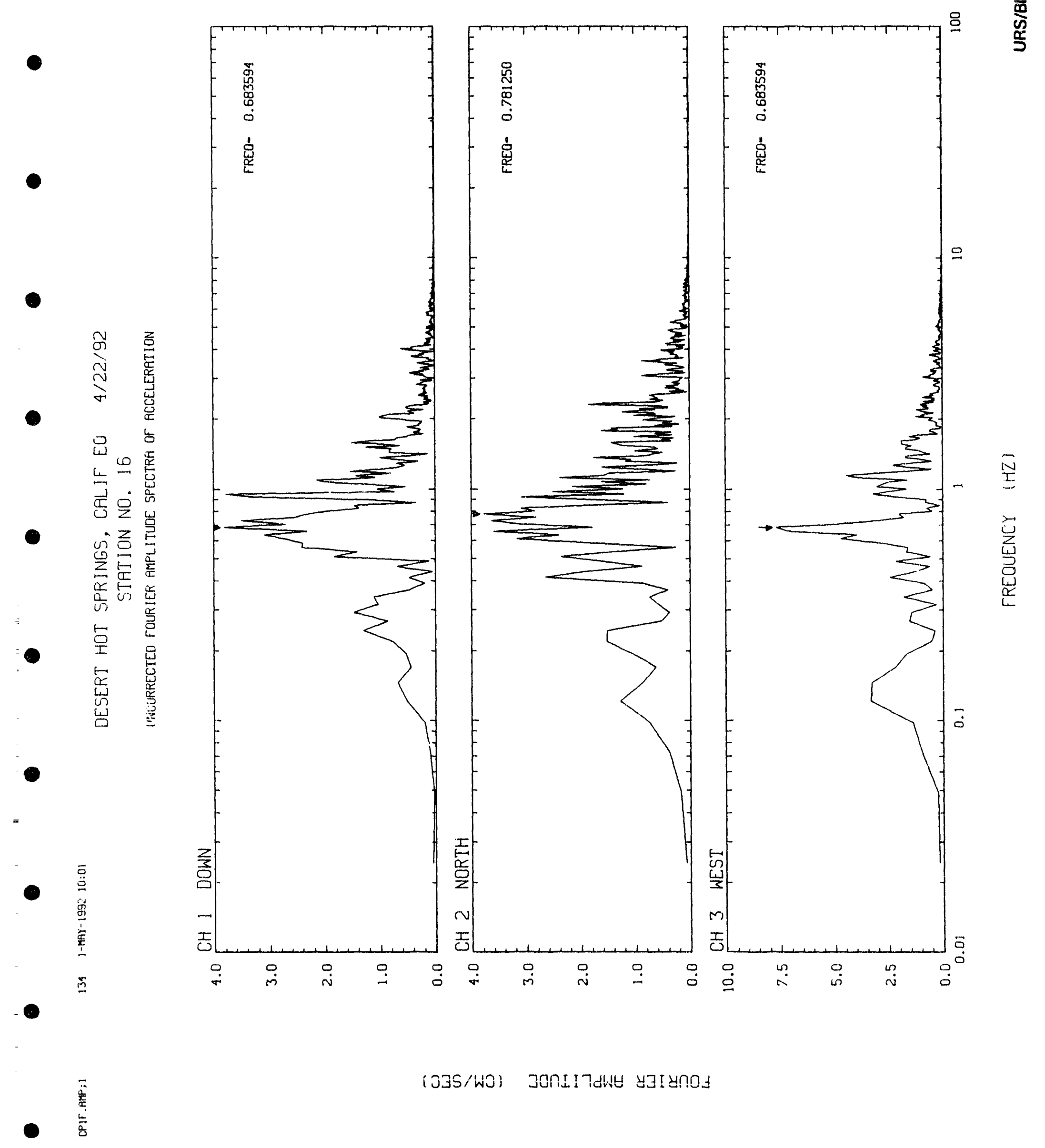



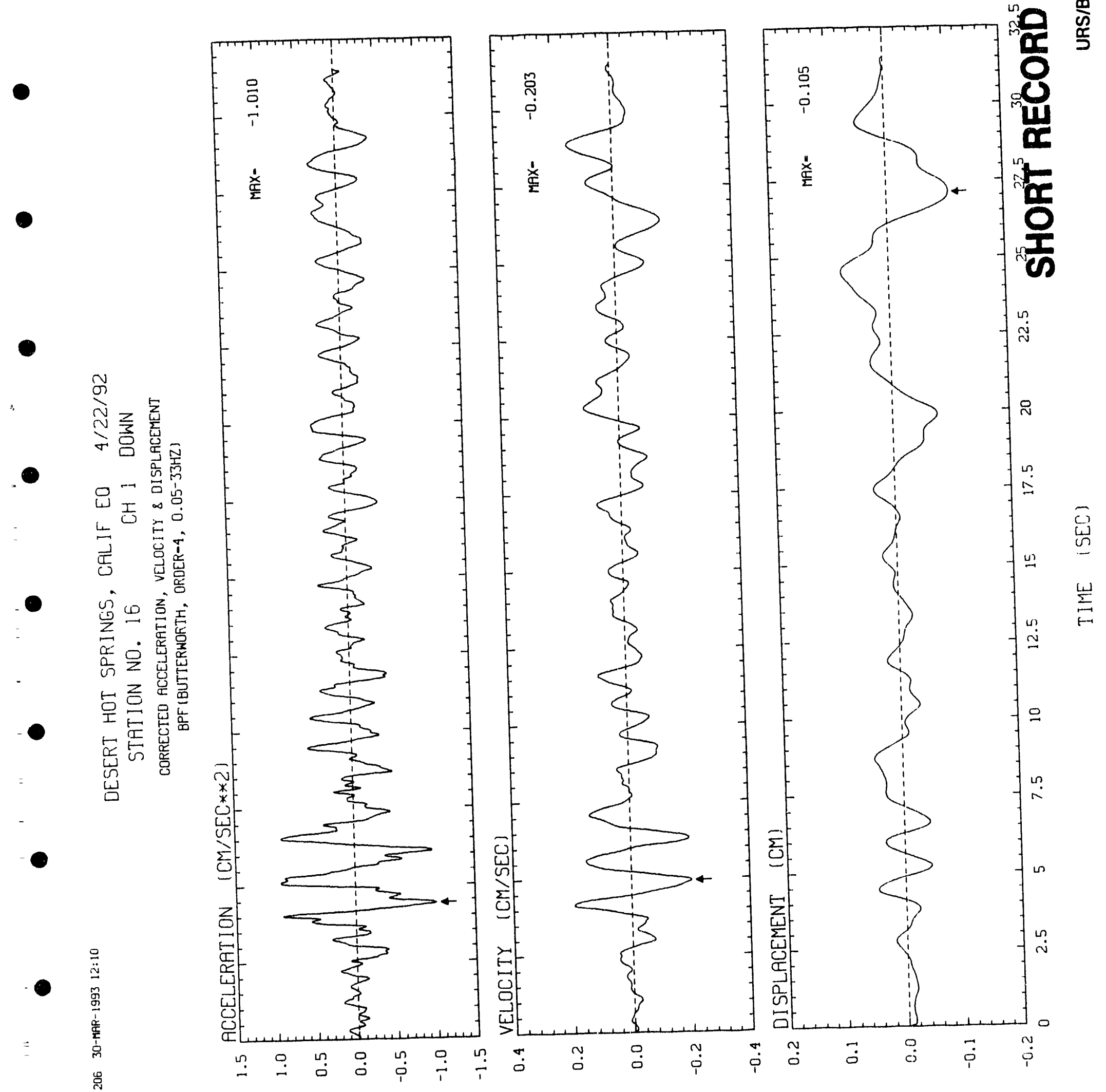

30กLI IdWH

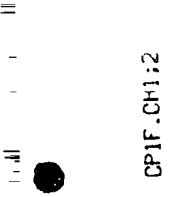




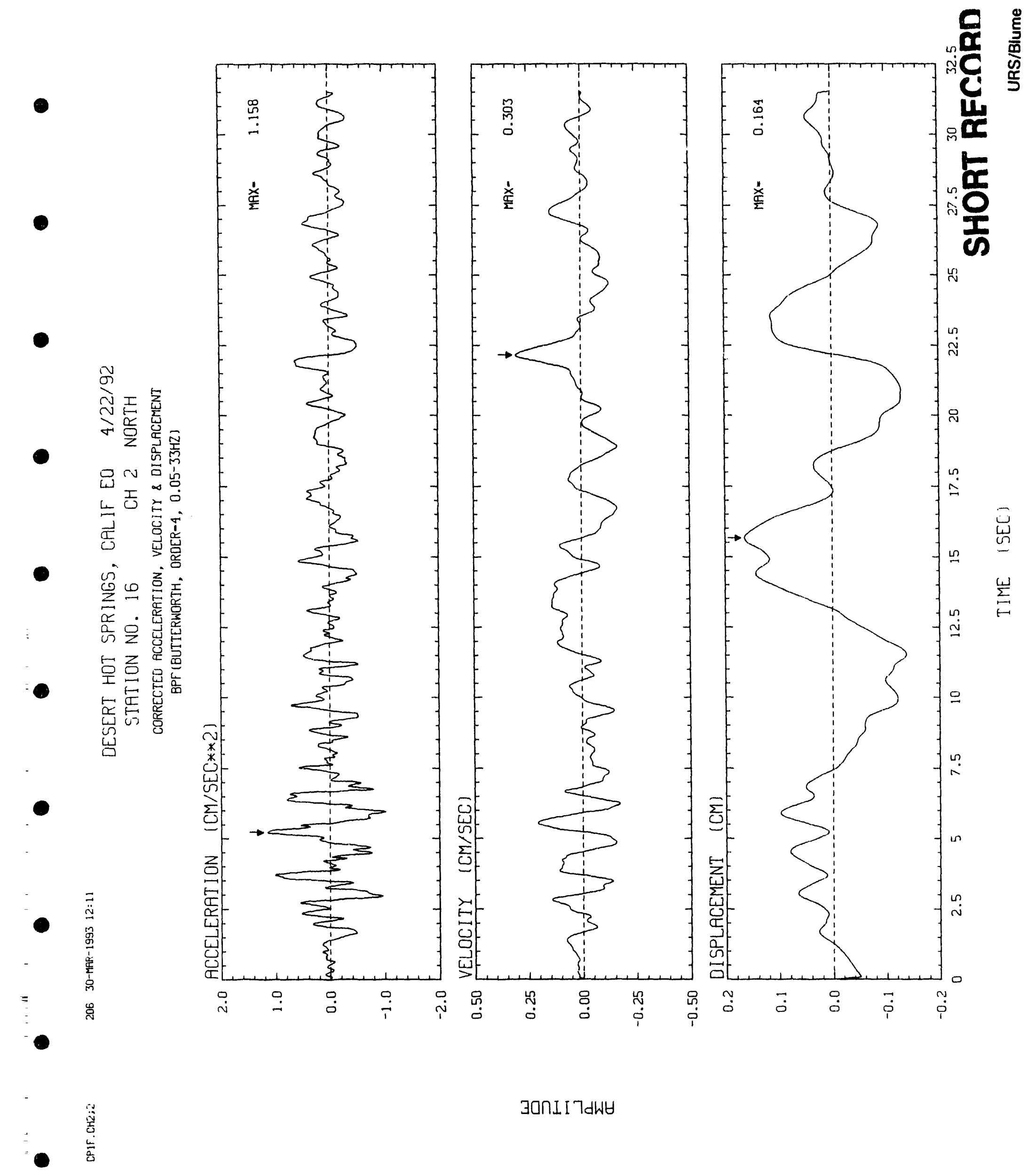




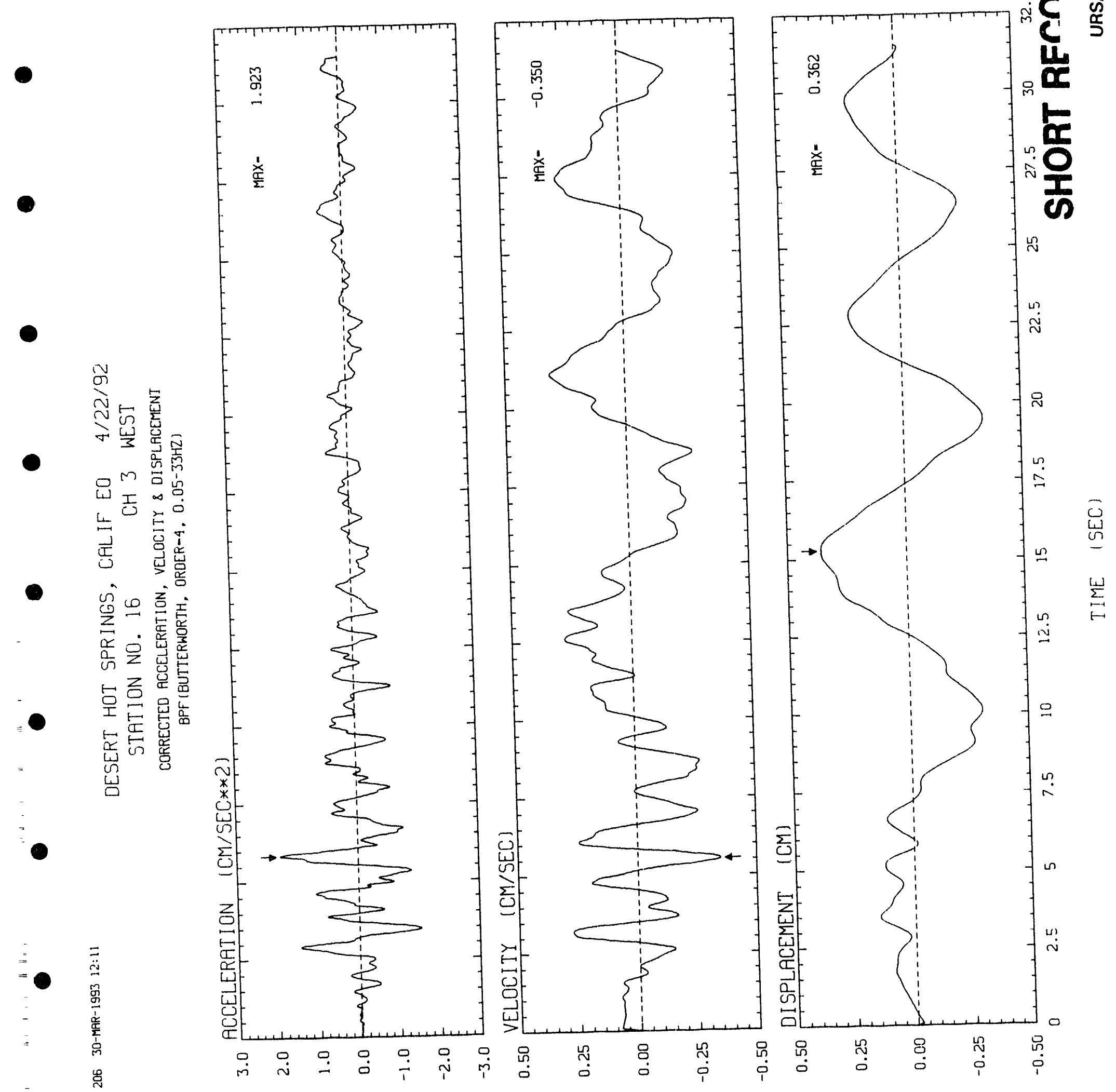

JUกL I 7dW

产 


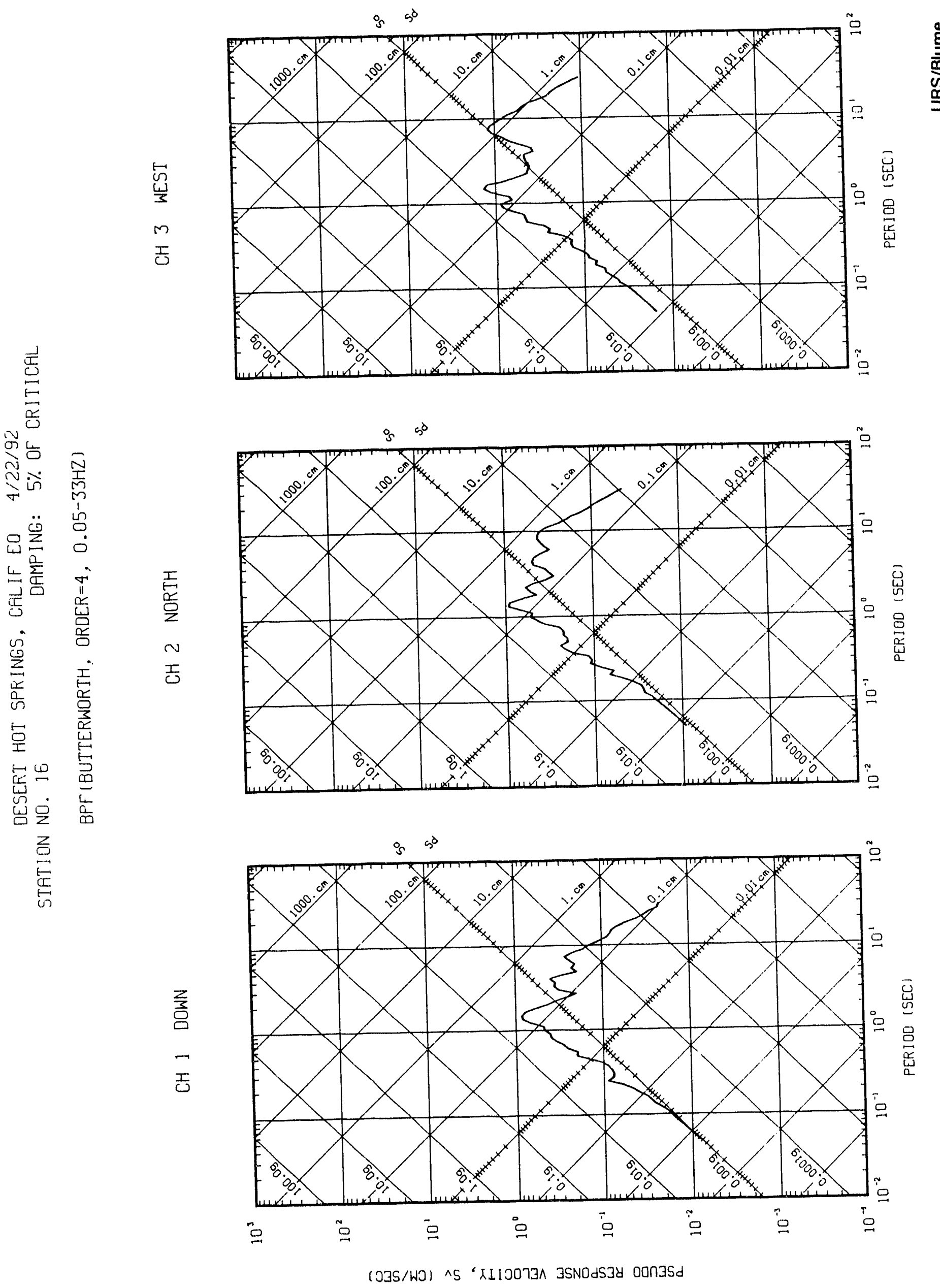


0

$\sum_{\frac{1}{5}}^{\frac{1}{\infty}}$

○

$N$

$r$

O

z

O

1

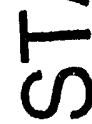

- 

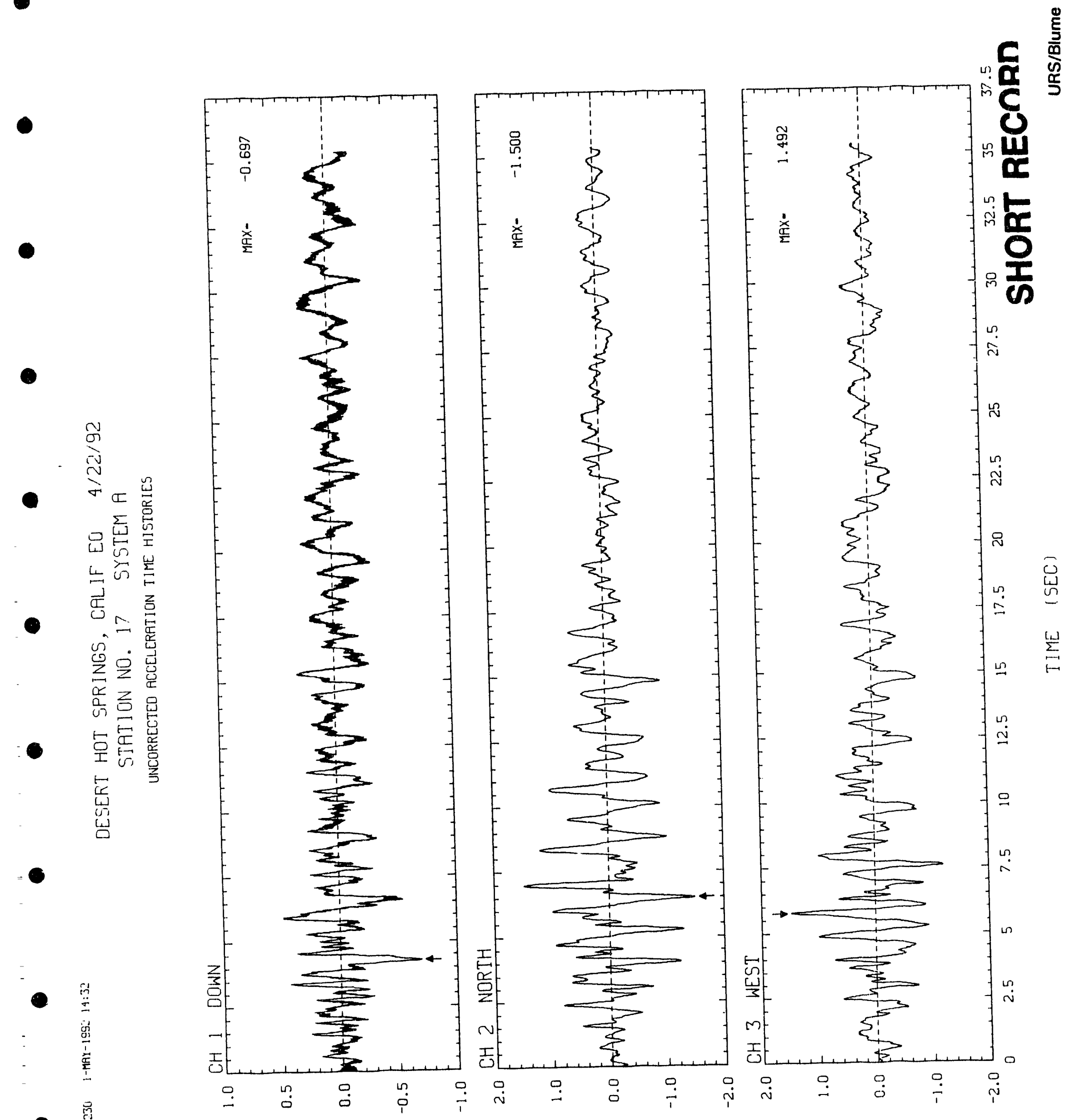

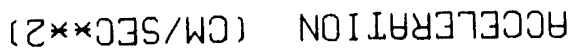

$\overrightarrow{\ddot{\ddot{\theta}}}$ 

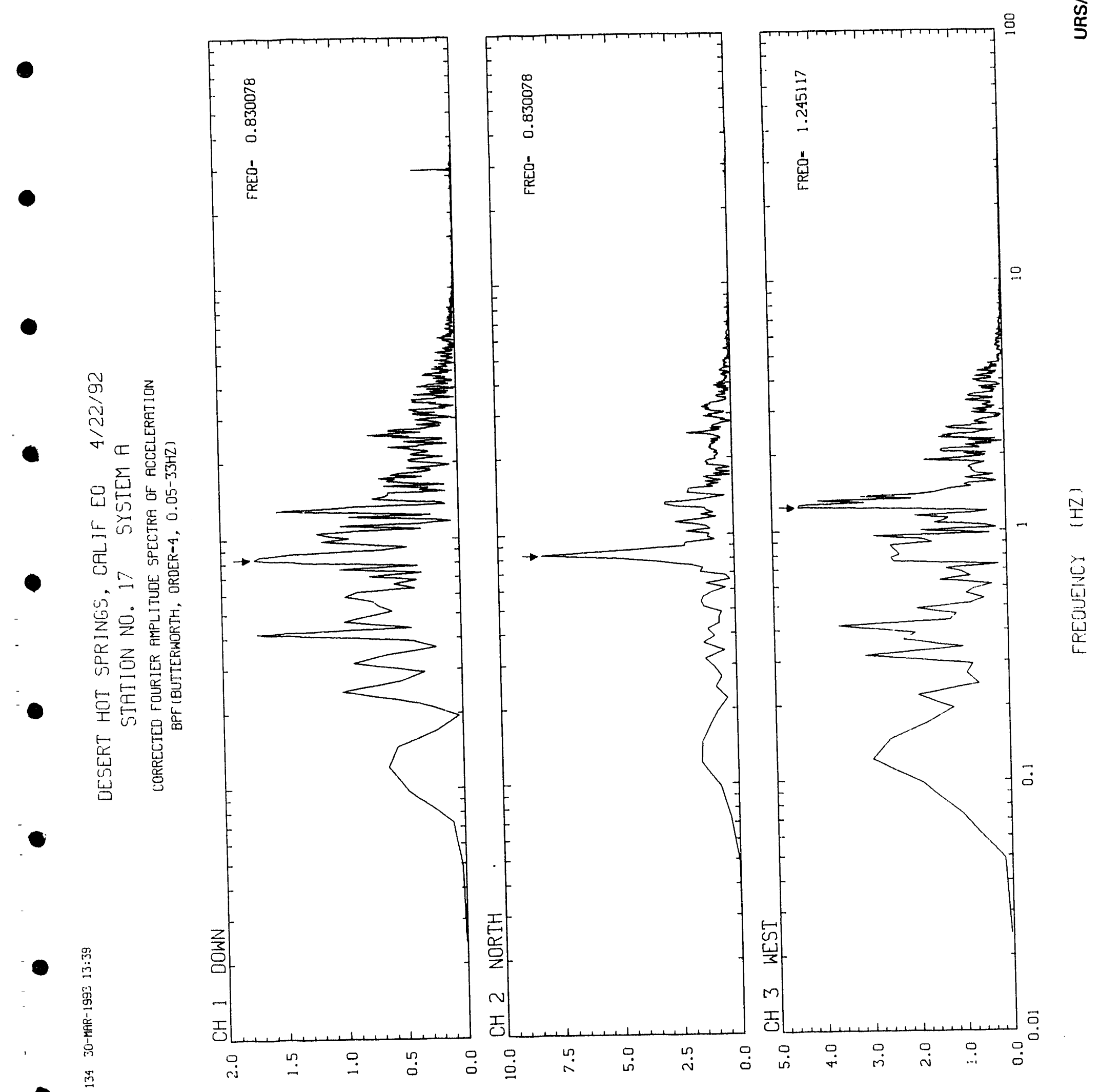


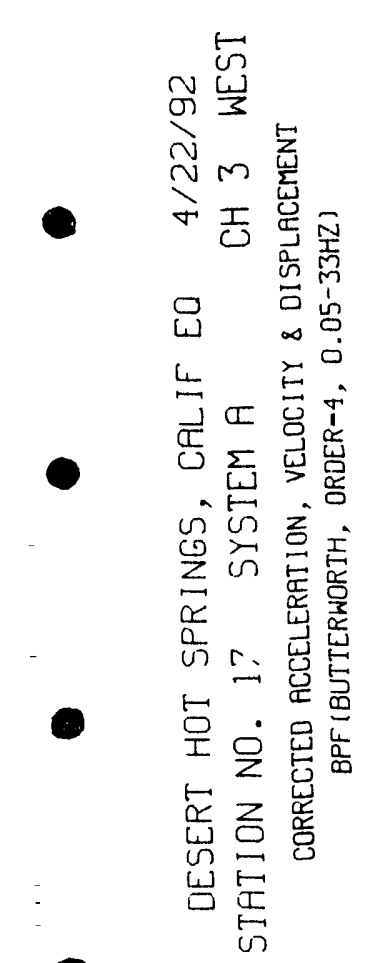

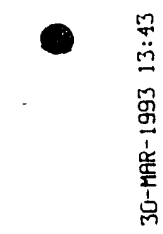

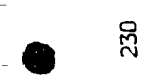
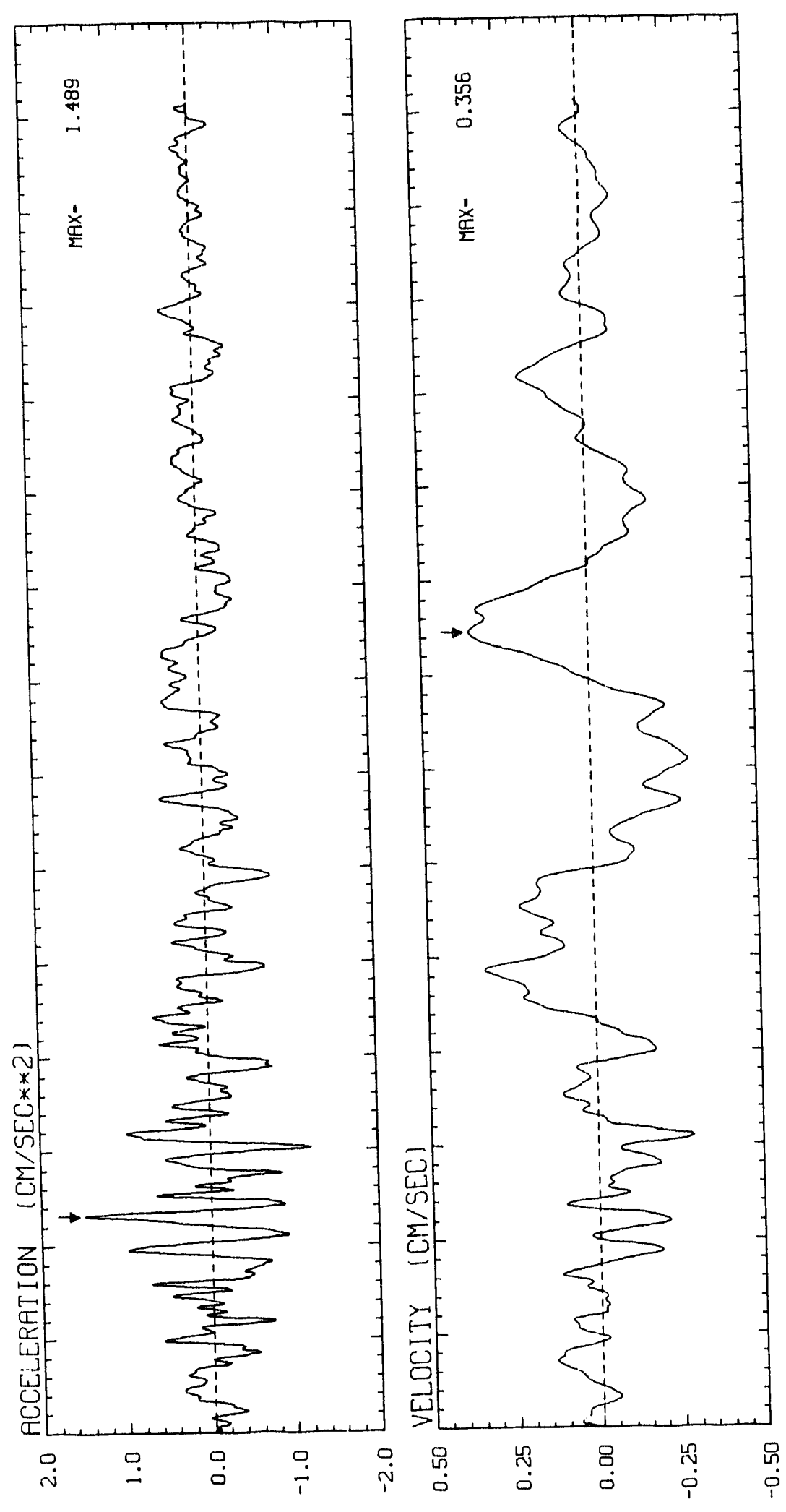

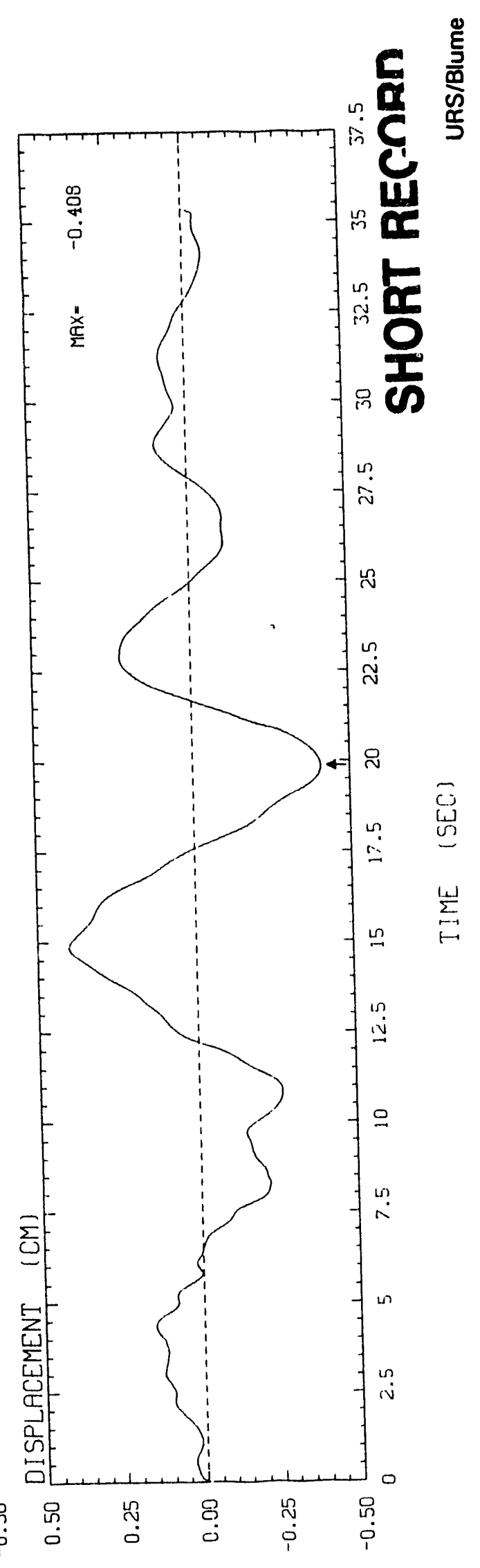

풓 

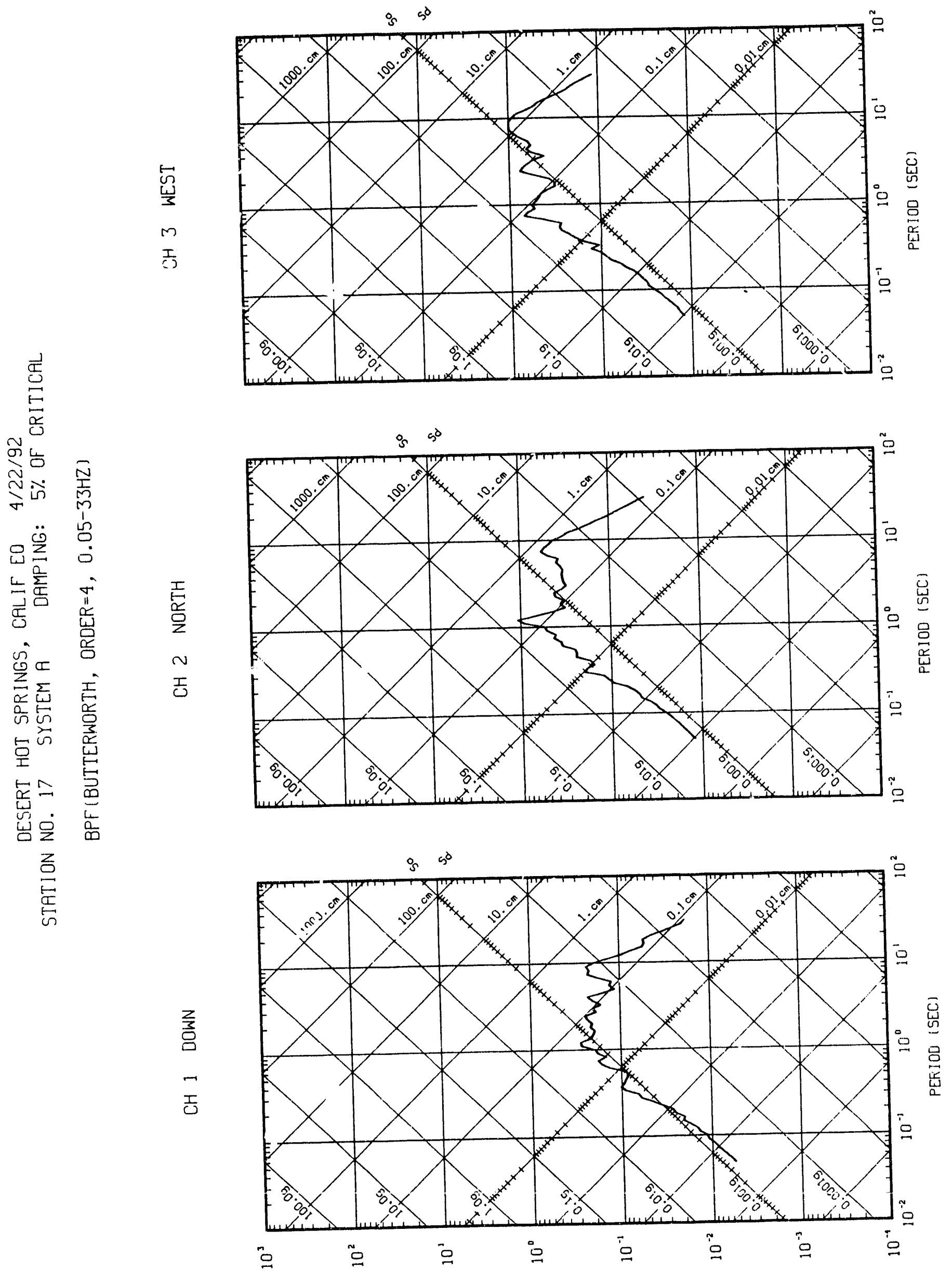
$m$

O

$\sum$

U

1

0

co

$N$

$r$

은

$z$

음

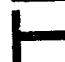

$\alpha$

10 

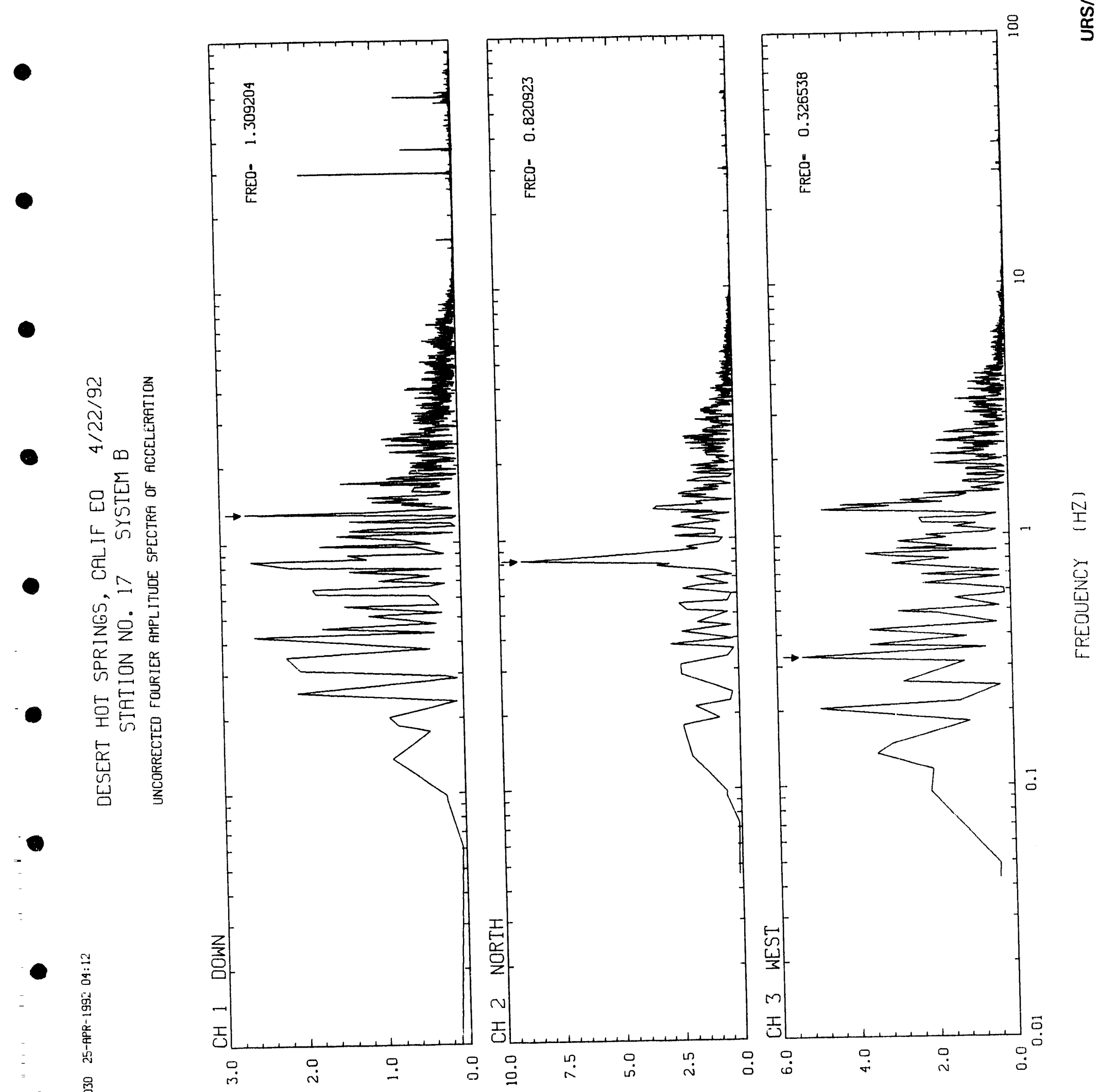

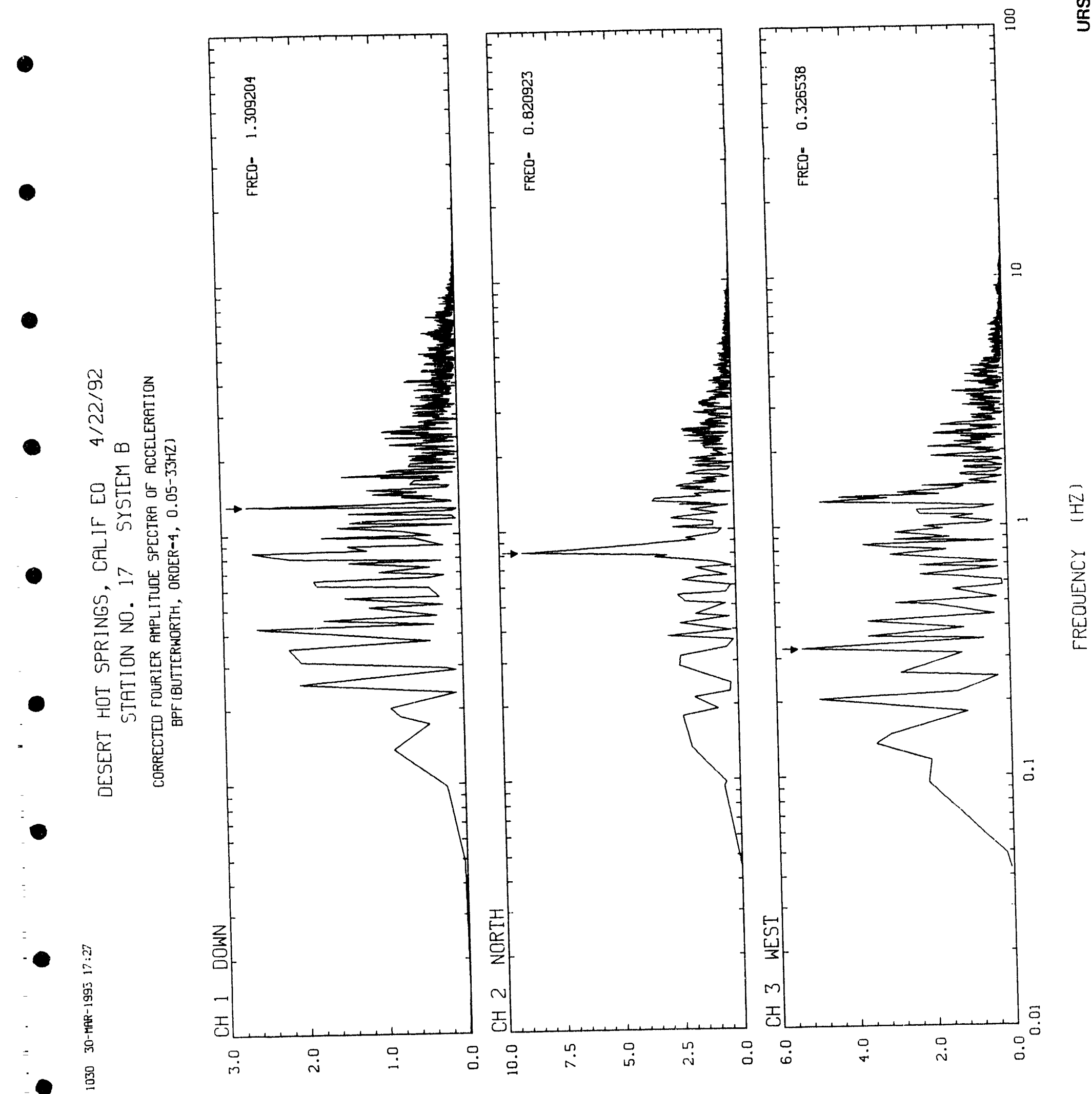

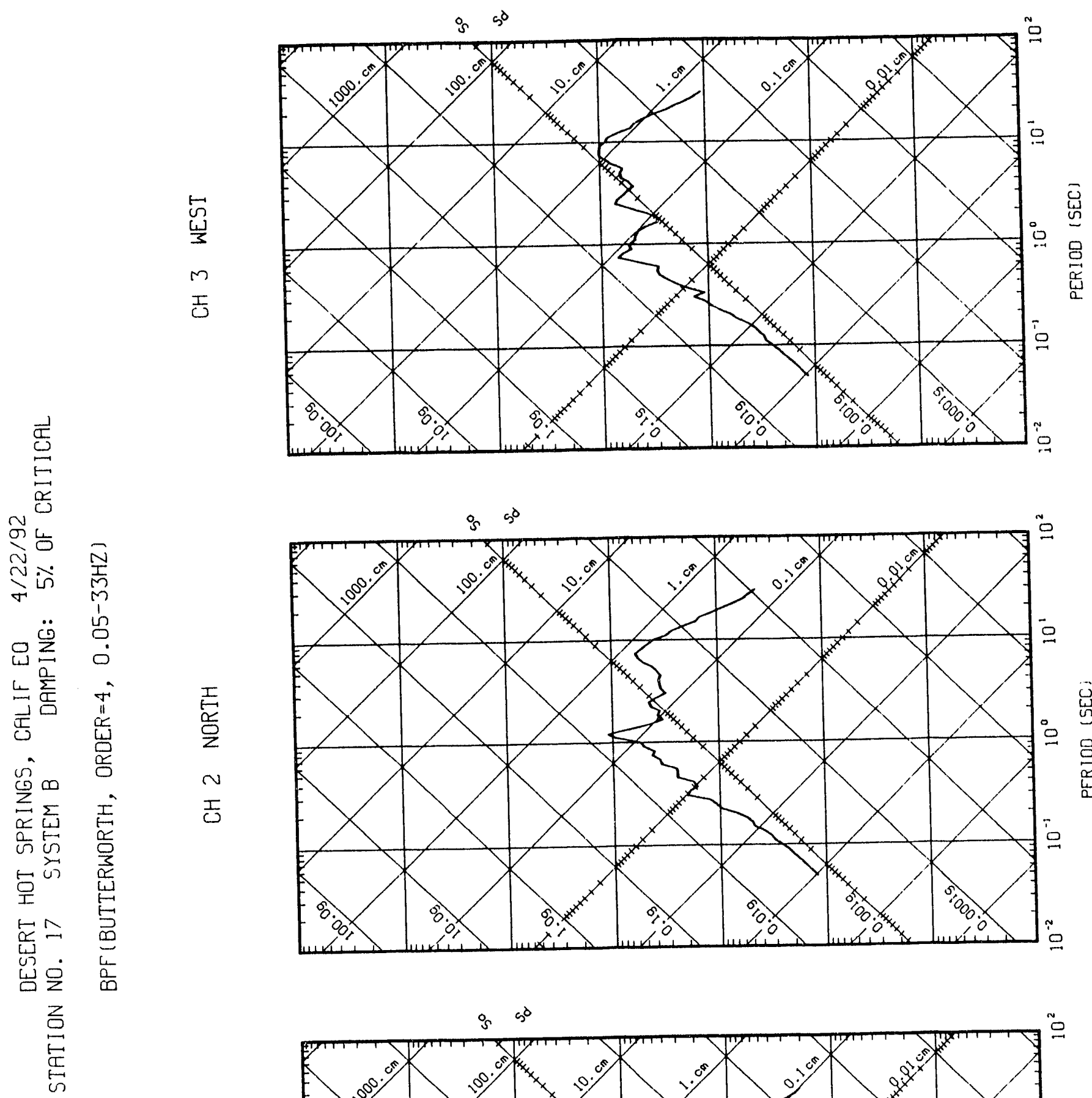

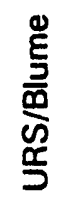
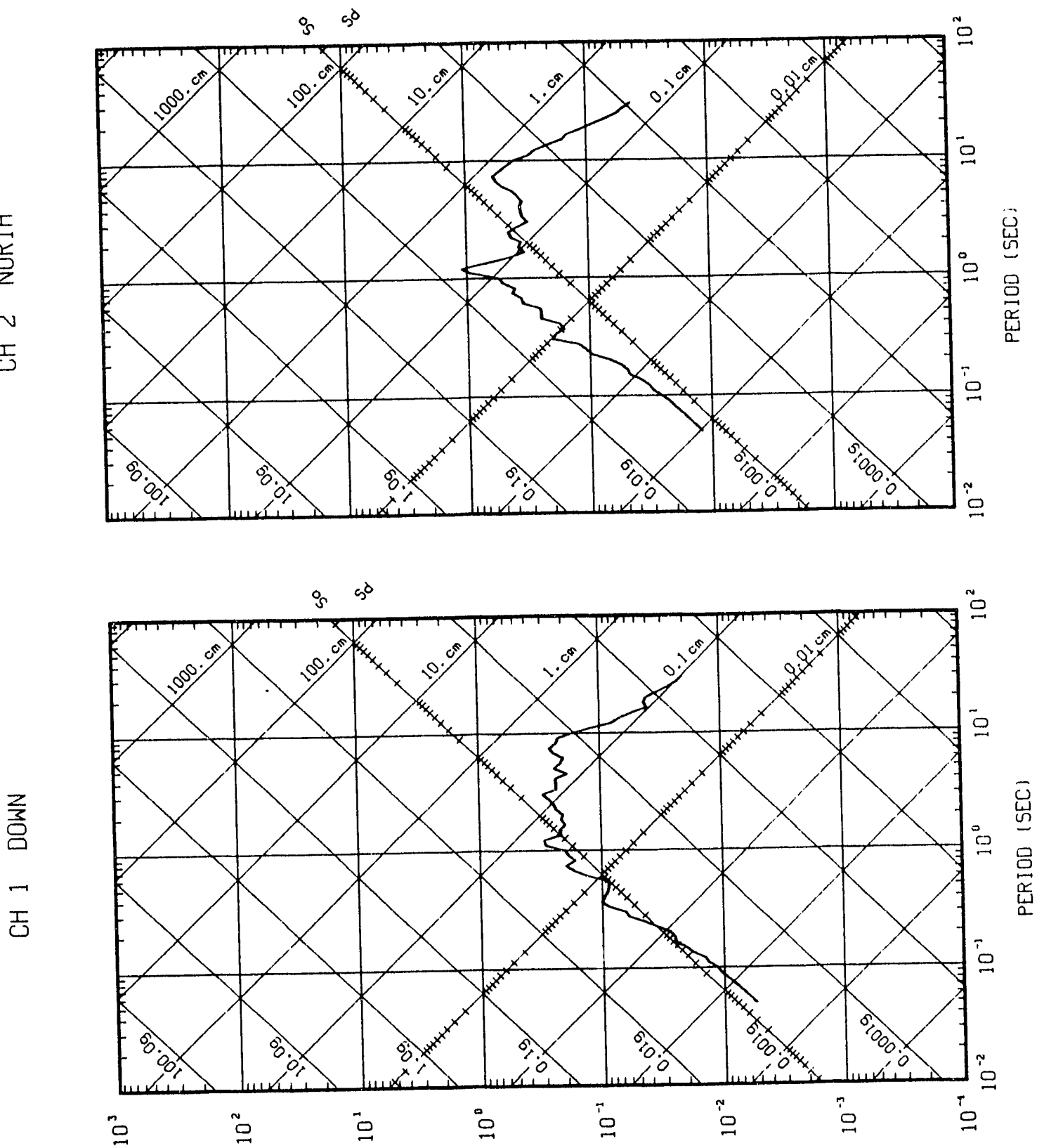

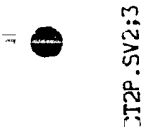

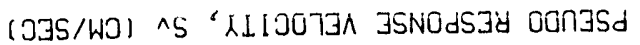


$\alpha$

$\sum_{0}^{\frac{5}{5}}$

$\infty$

$r$

0

-

$\uparrow$

0 

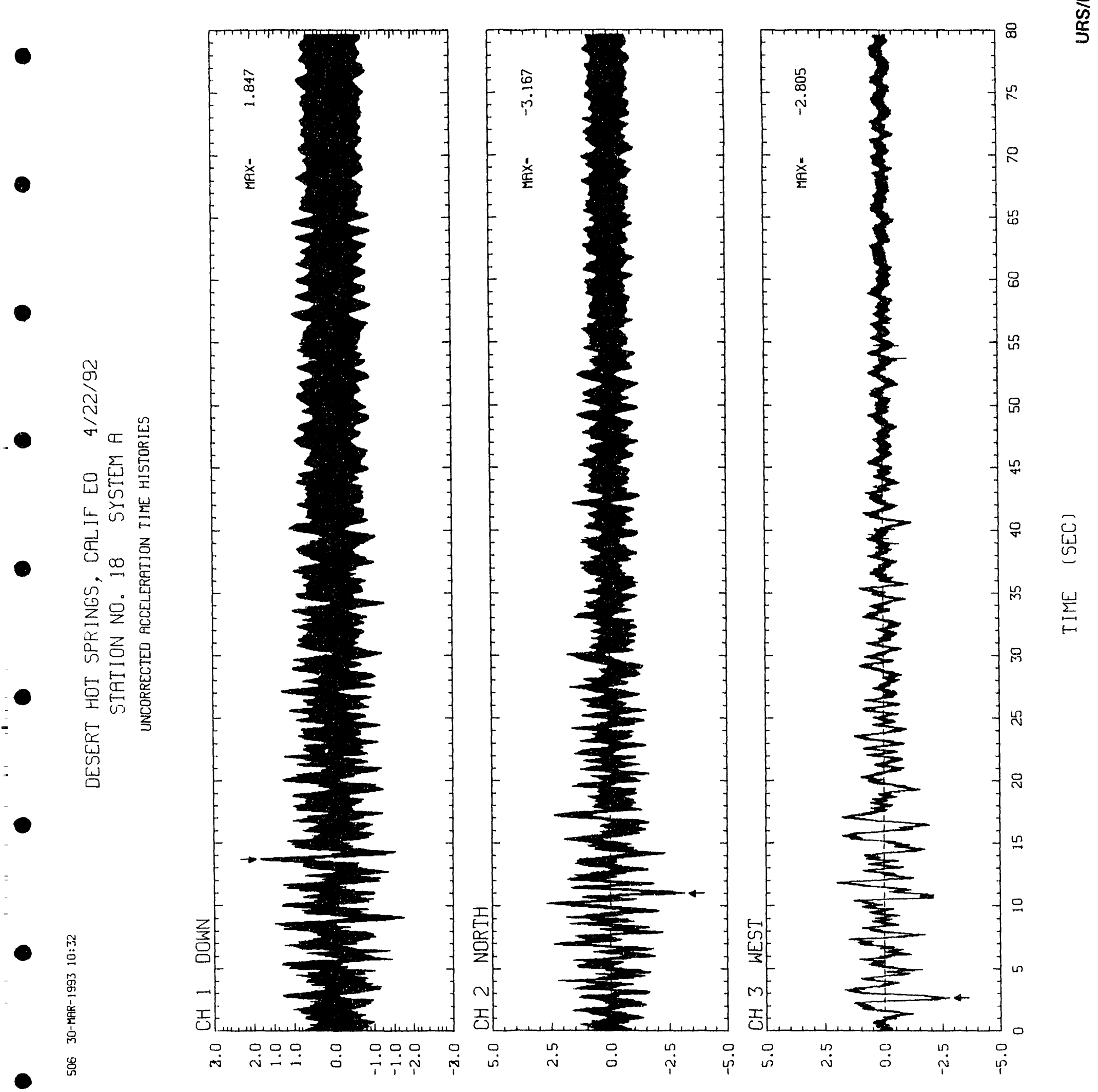

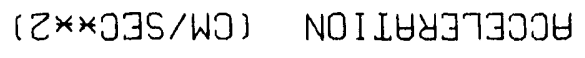




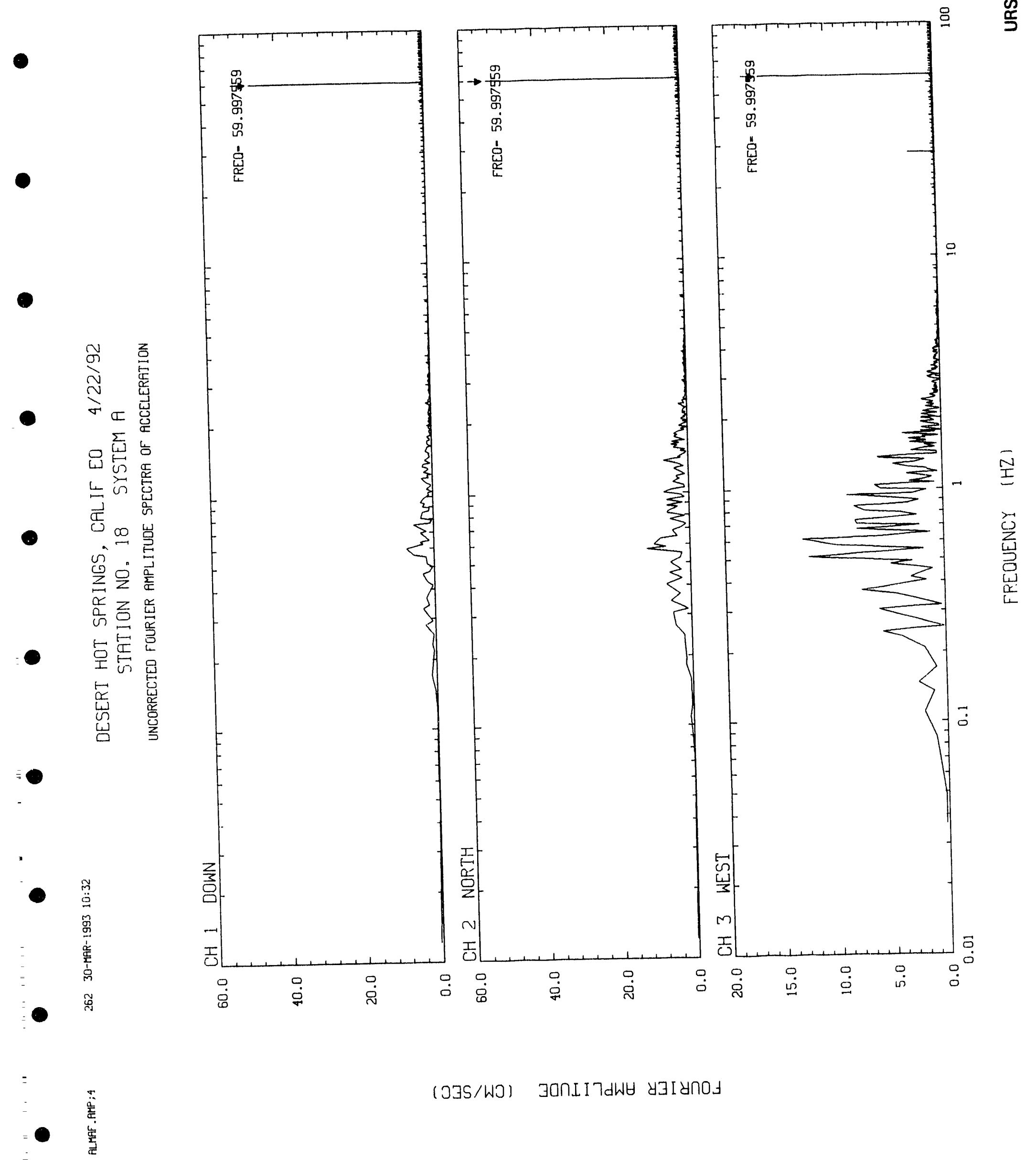




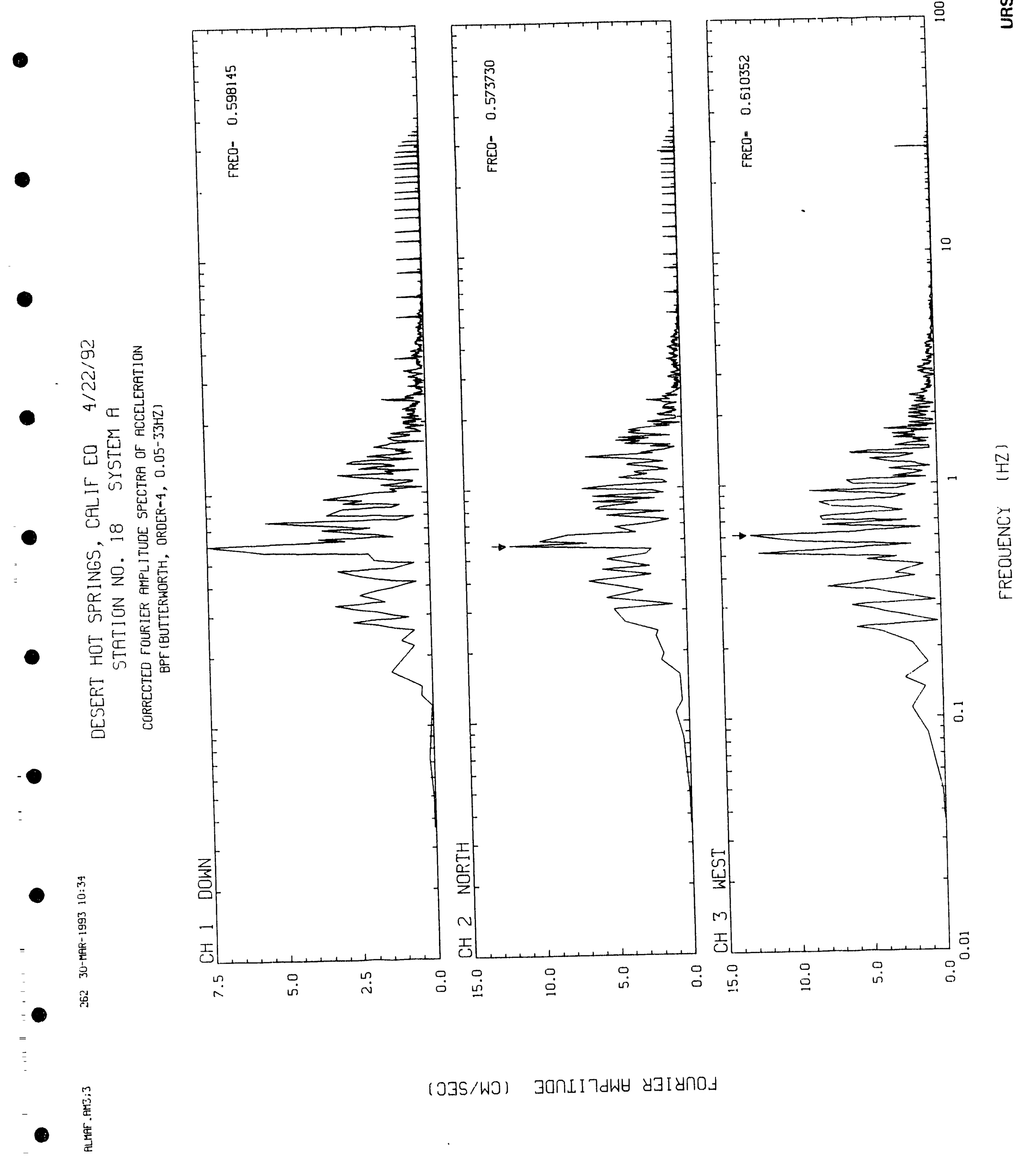



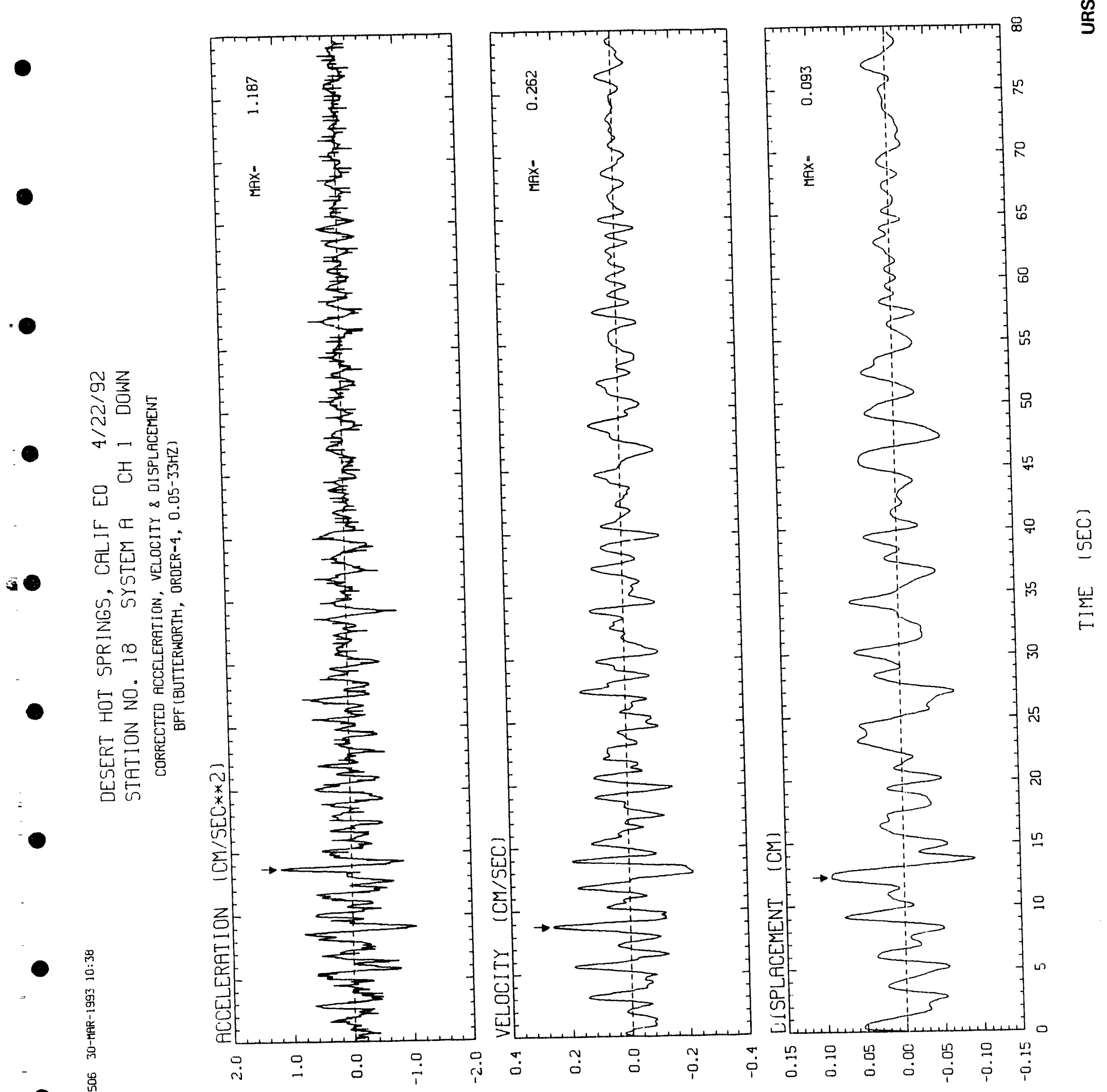

$\stackrel{?}{\stackrel{7}{5}}$ 


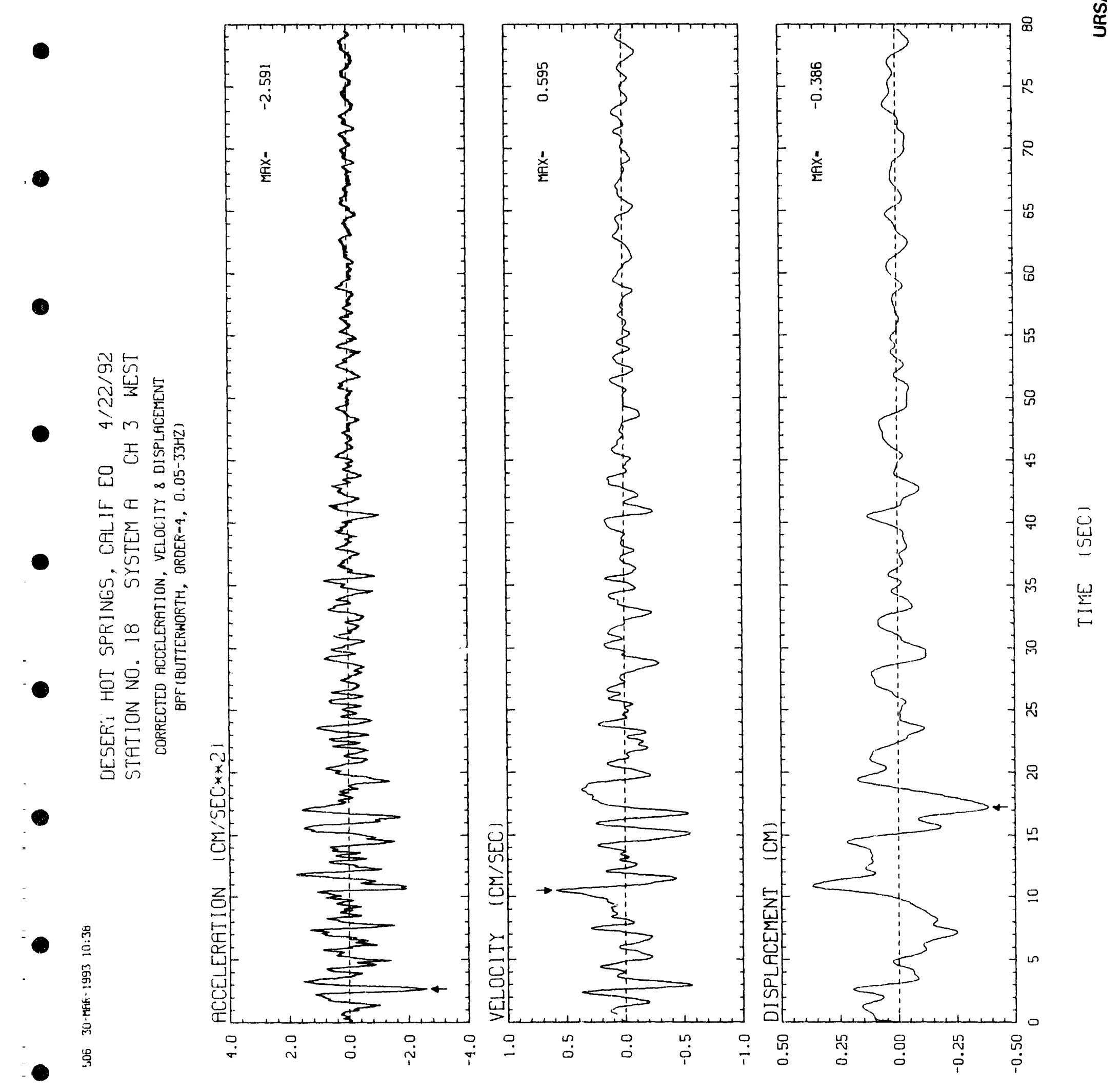



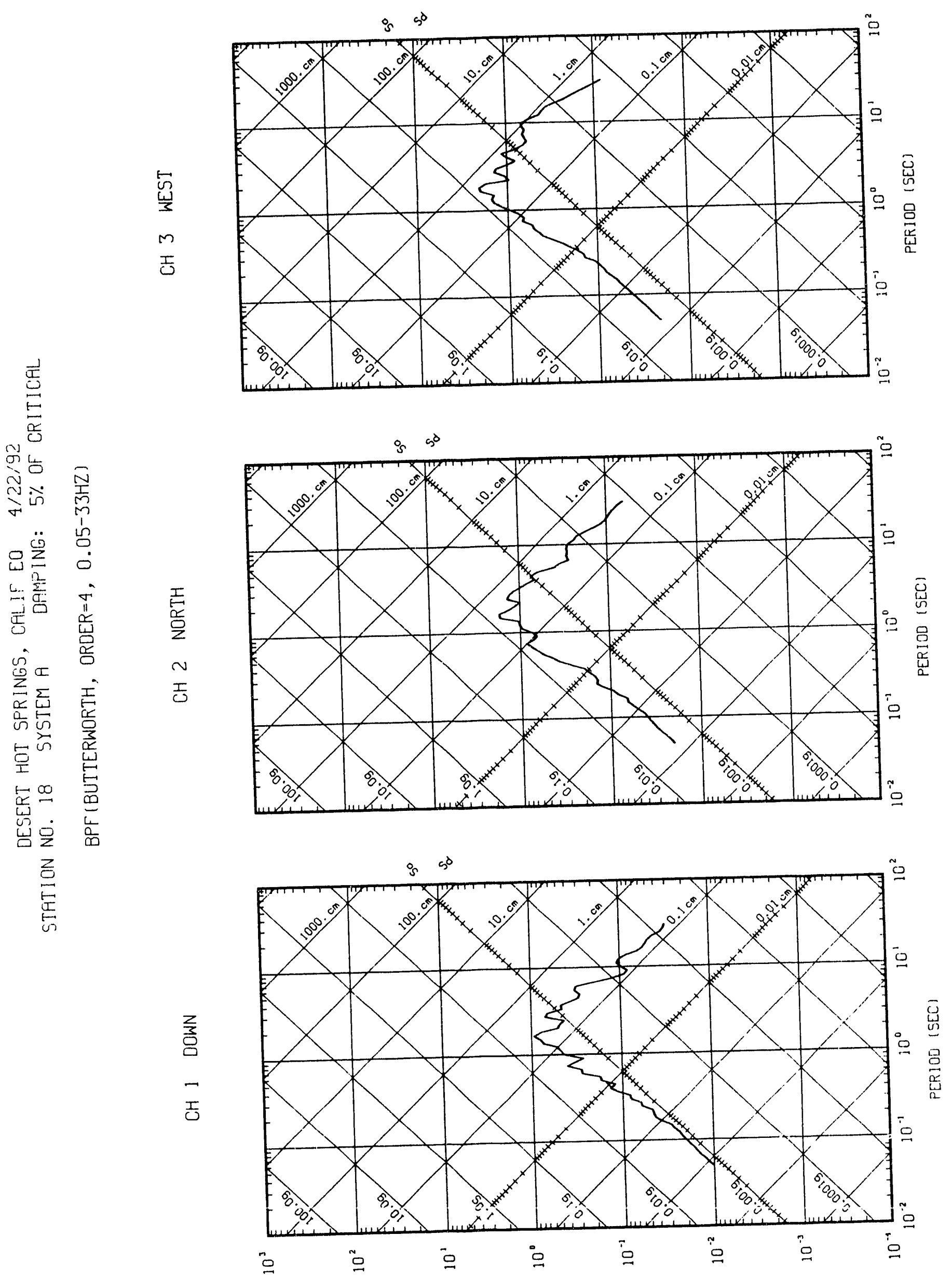

(O3S/WOI ^S 'xIIJOTJA JSNOdSJY OONZSd 
$\infty$ $r$ $\stackrel{0}{z}$

z

O

$E$

$\varangle$

的

$=$
$=$
$=$
$=$
$=$ 

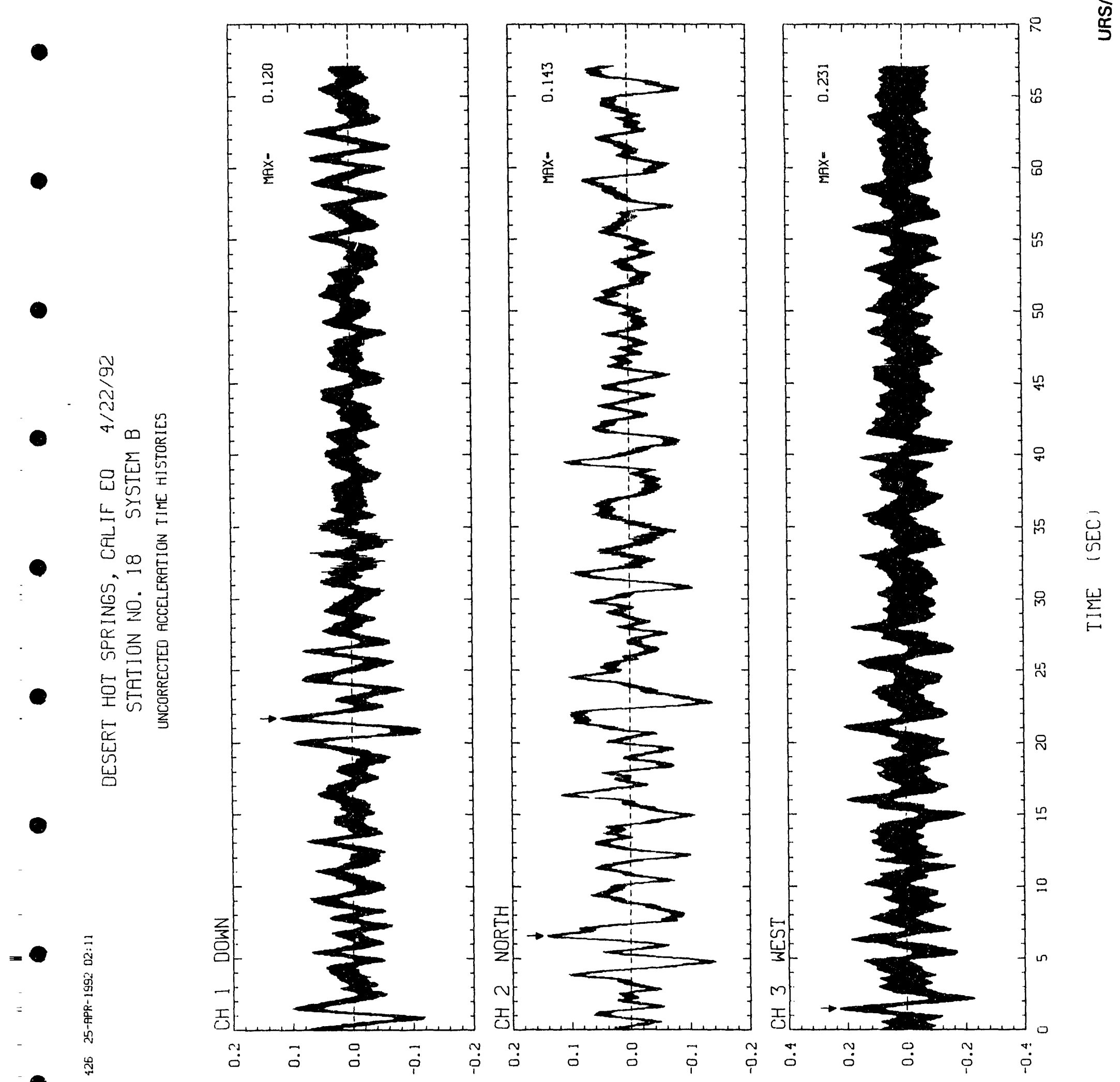

(2**3ヨS/WJ) NDIIH4ヨา 

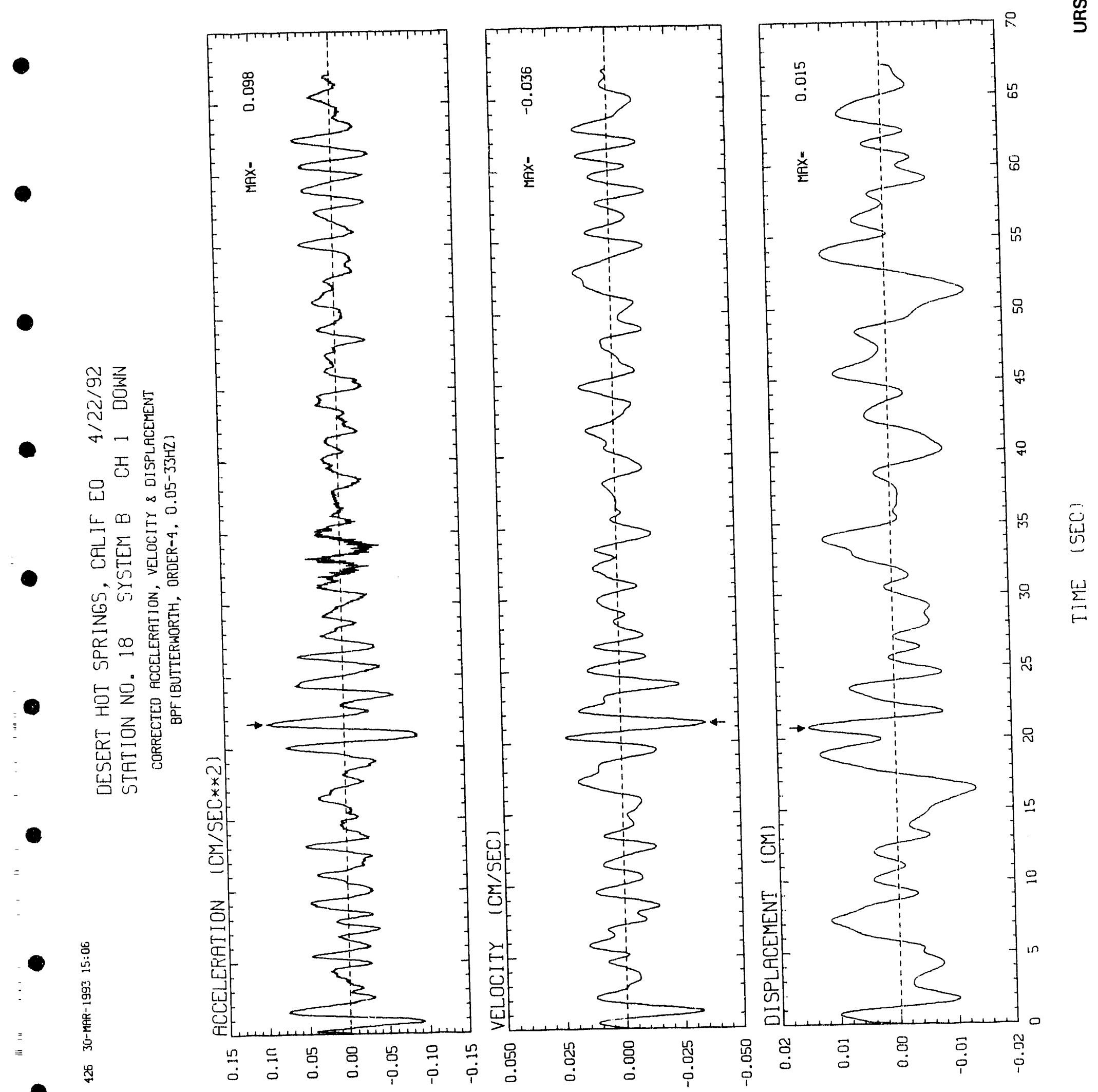


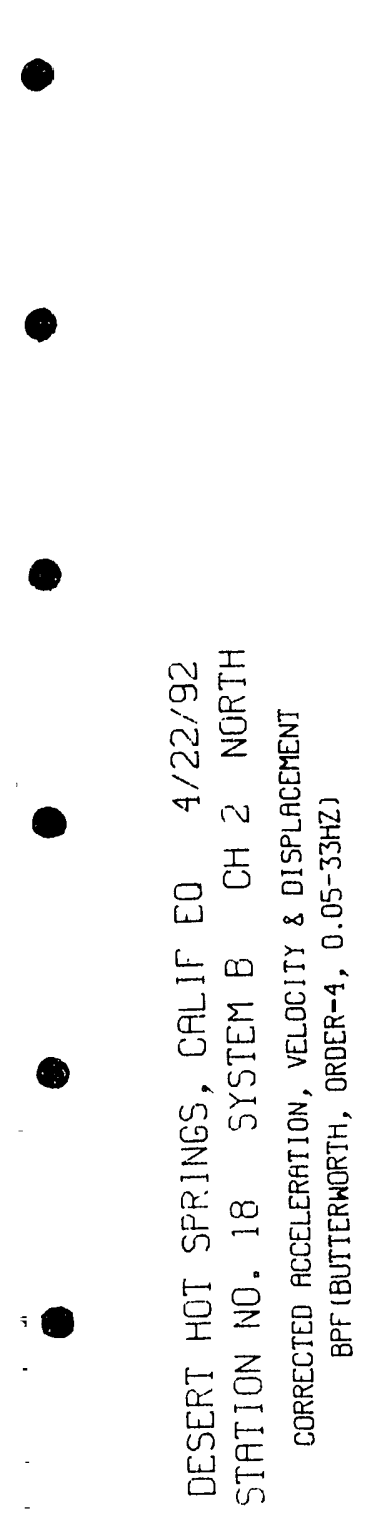

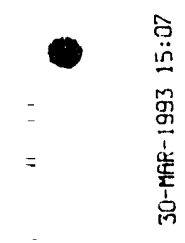
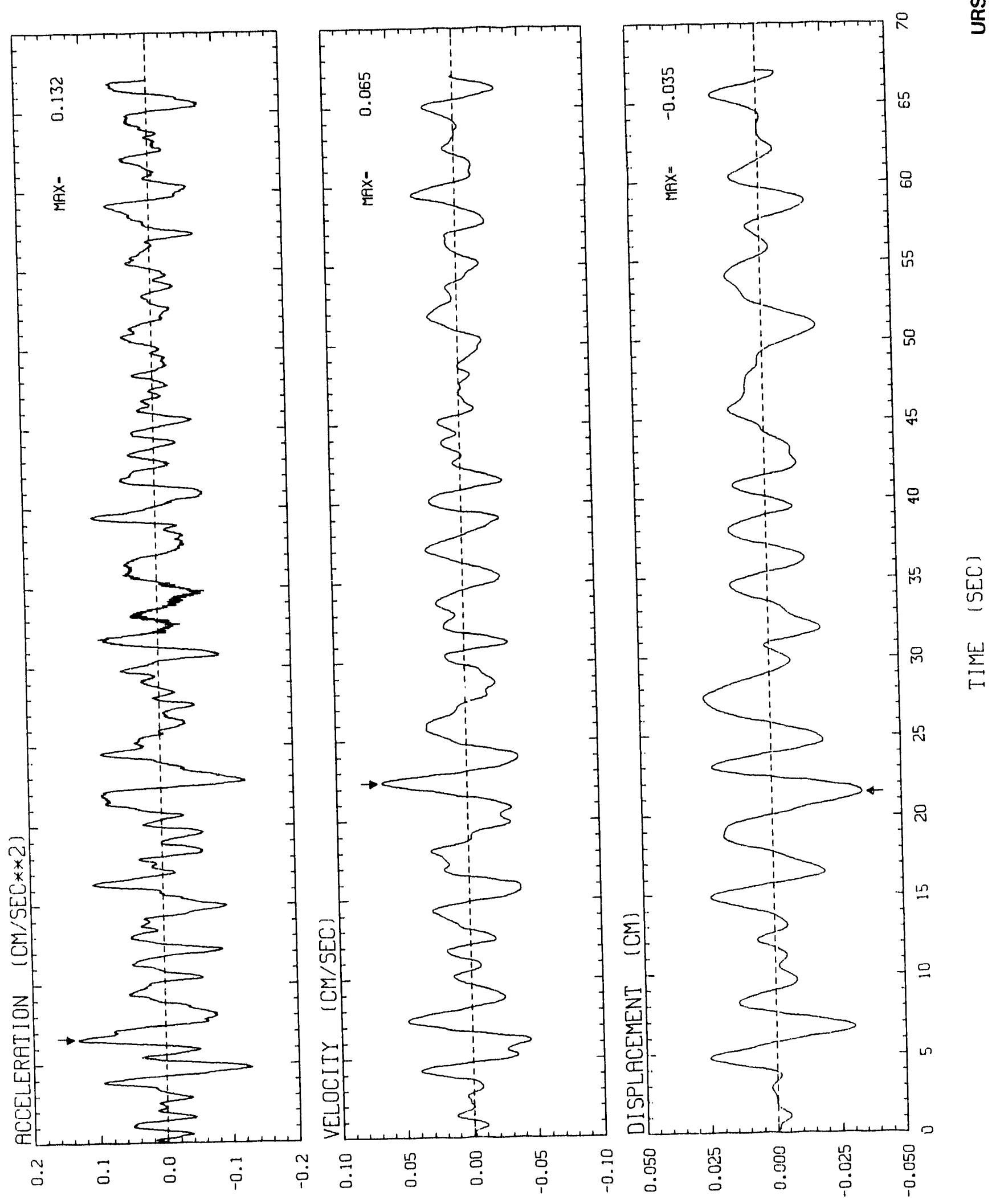


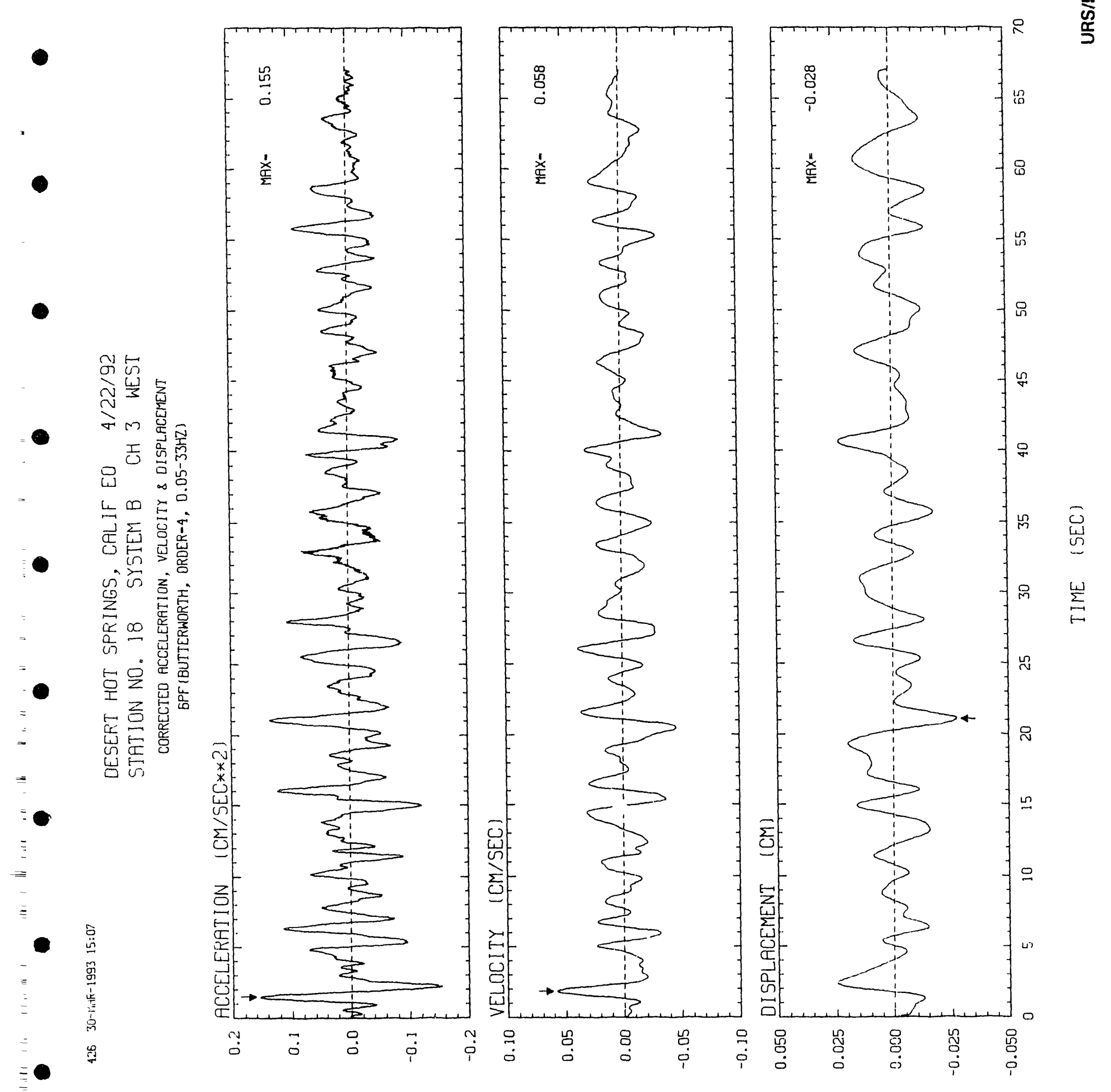



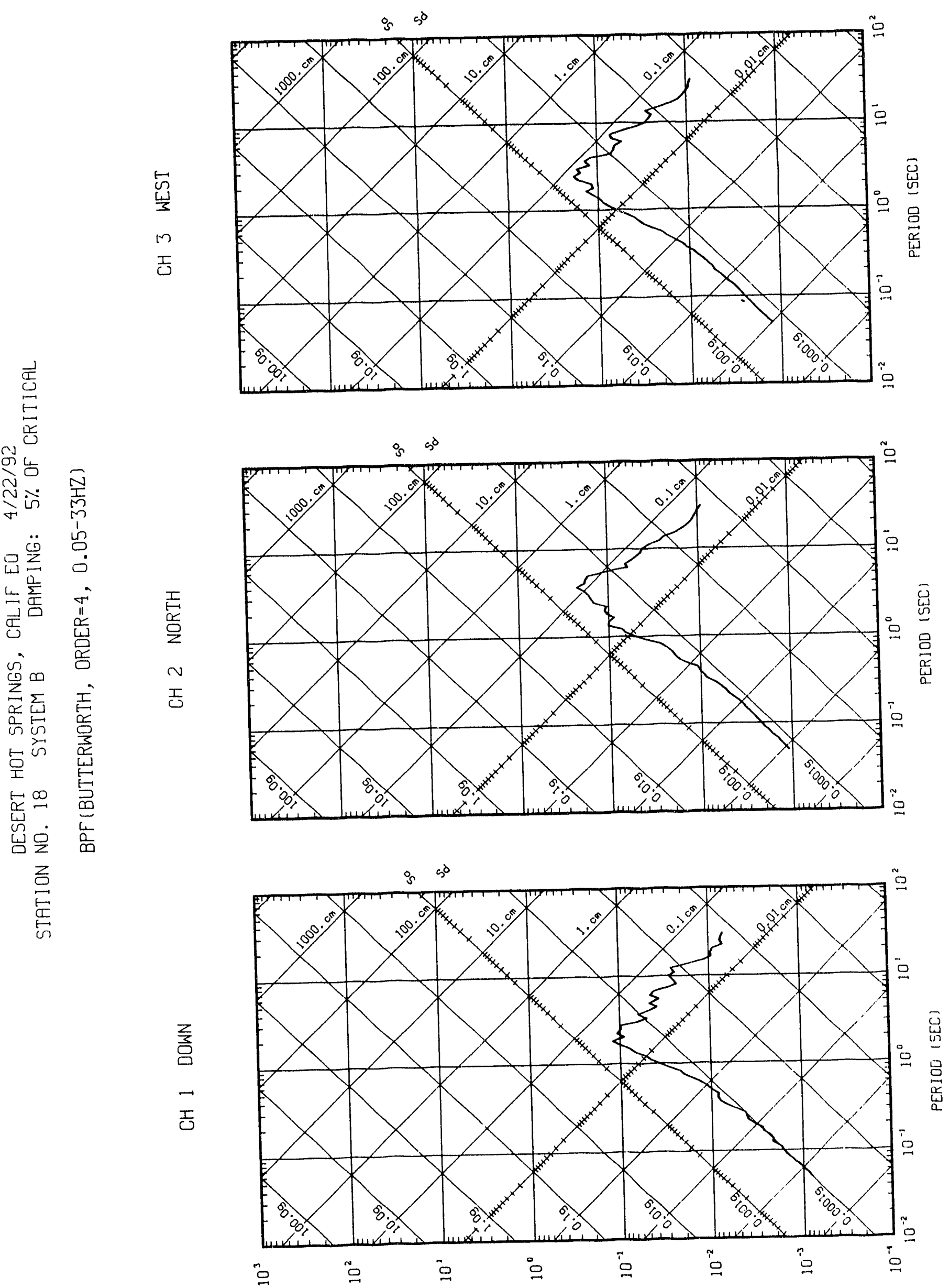
0

○

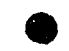

○

0

0 

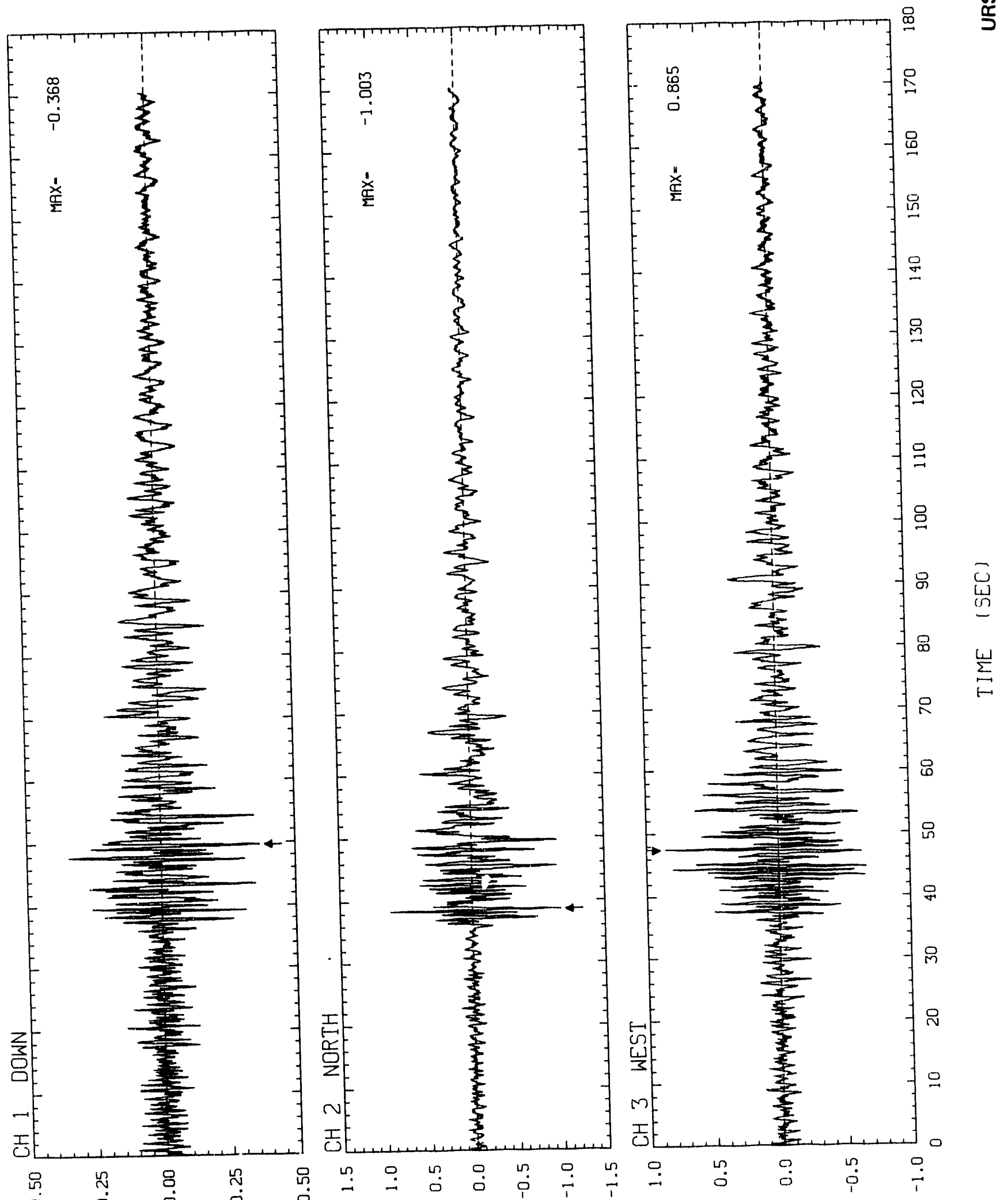

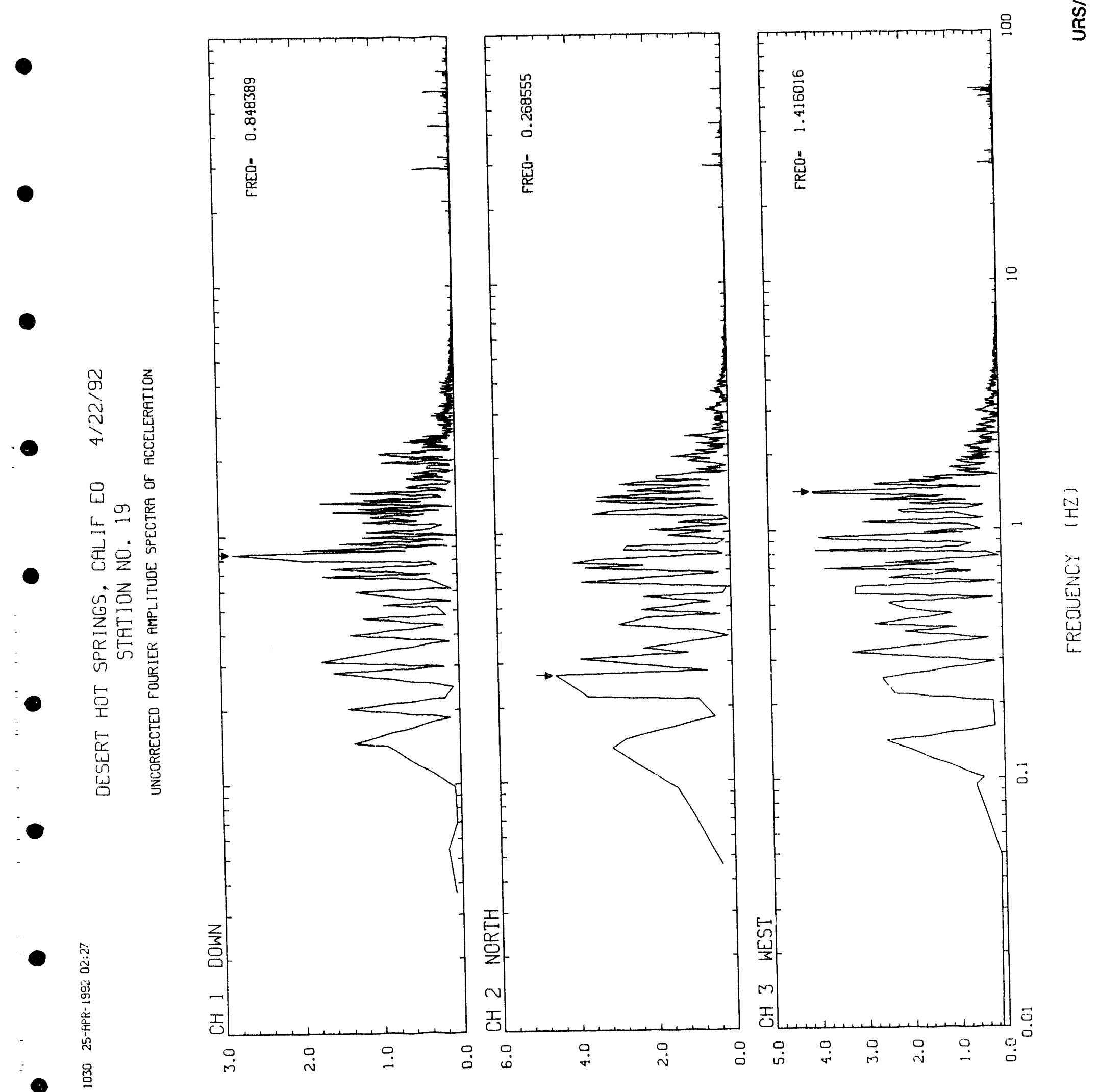


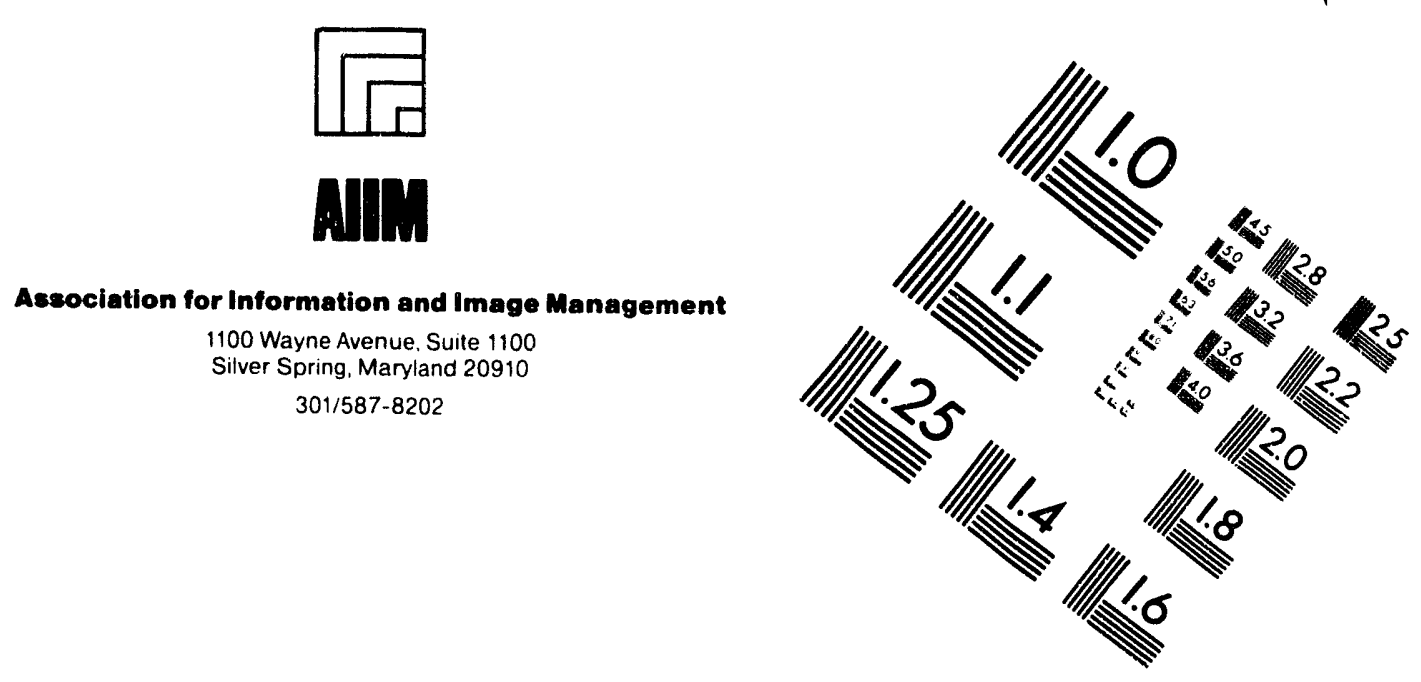

\section{Centimeter}

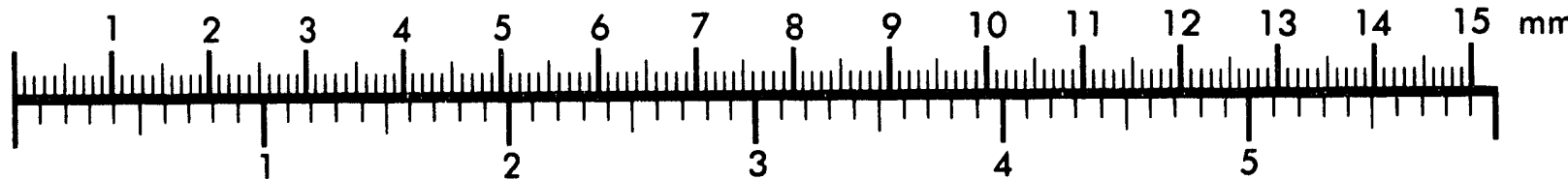
Inches
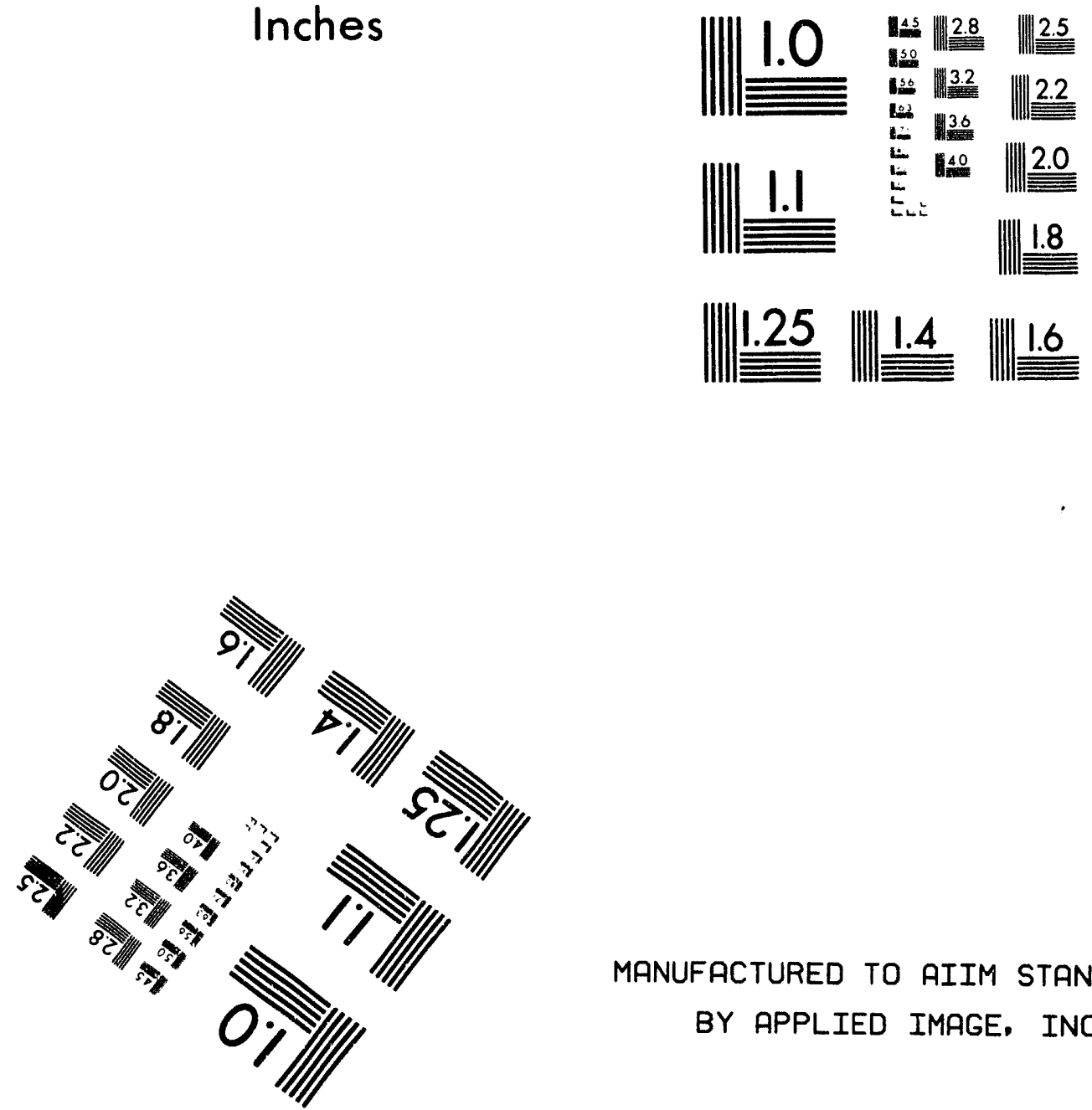

MANUFACTURED TO AIIM STANDARDS BY APPLIED IMAGE, INC.

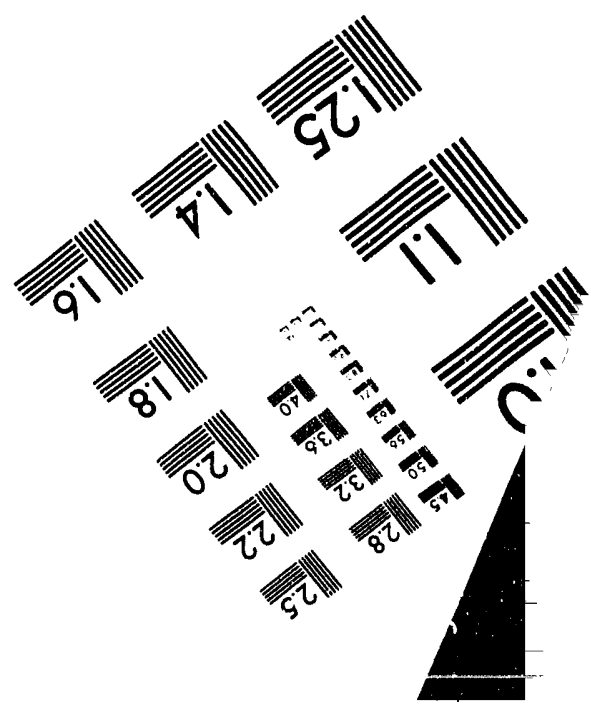



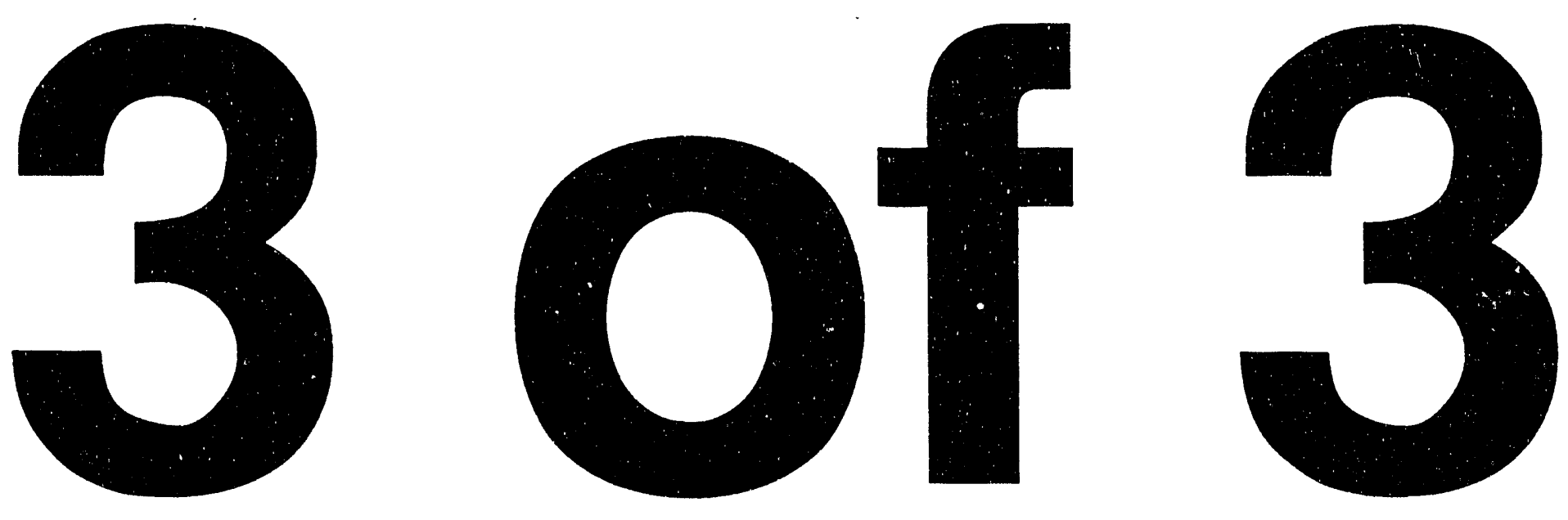


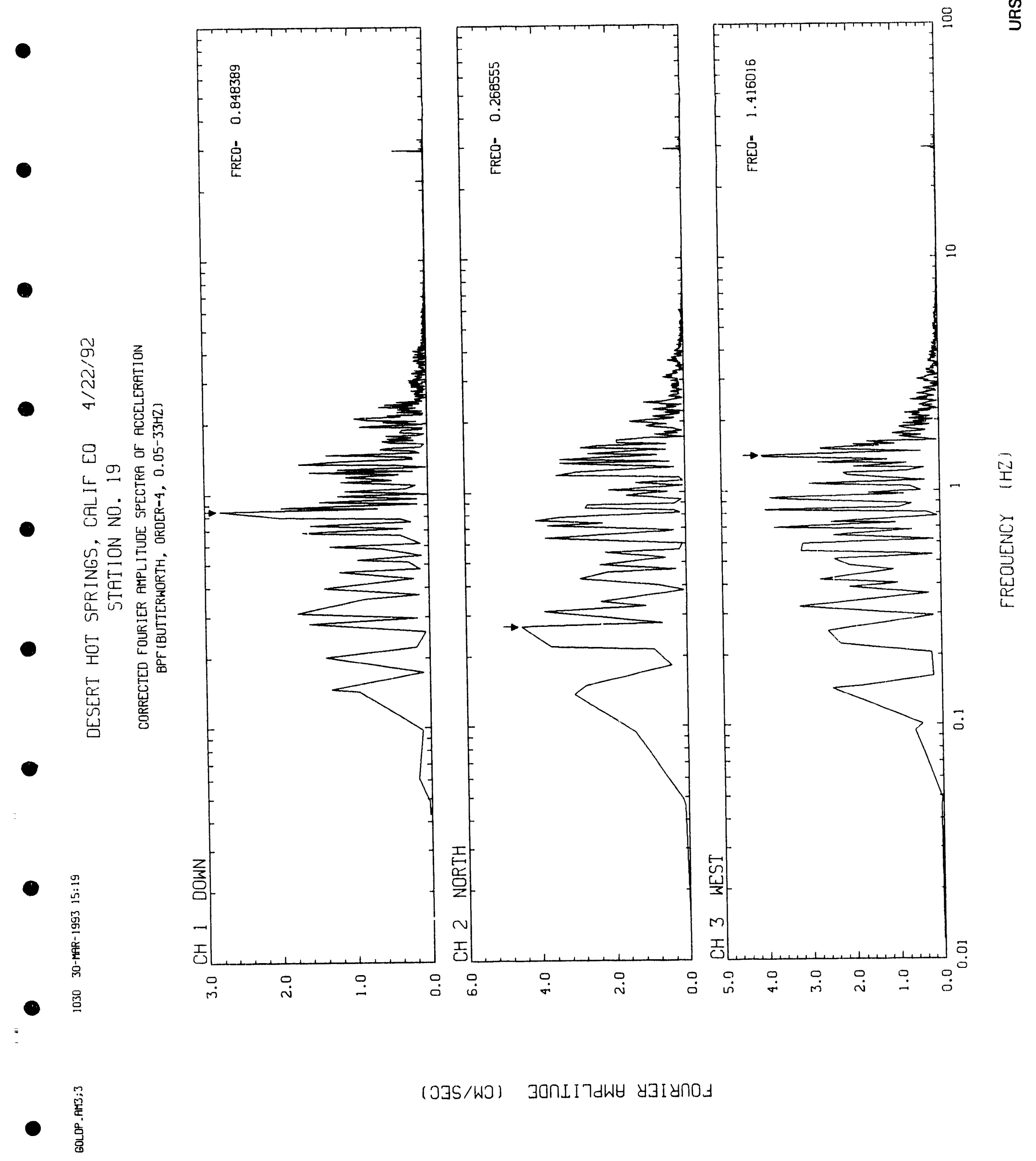



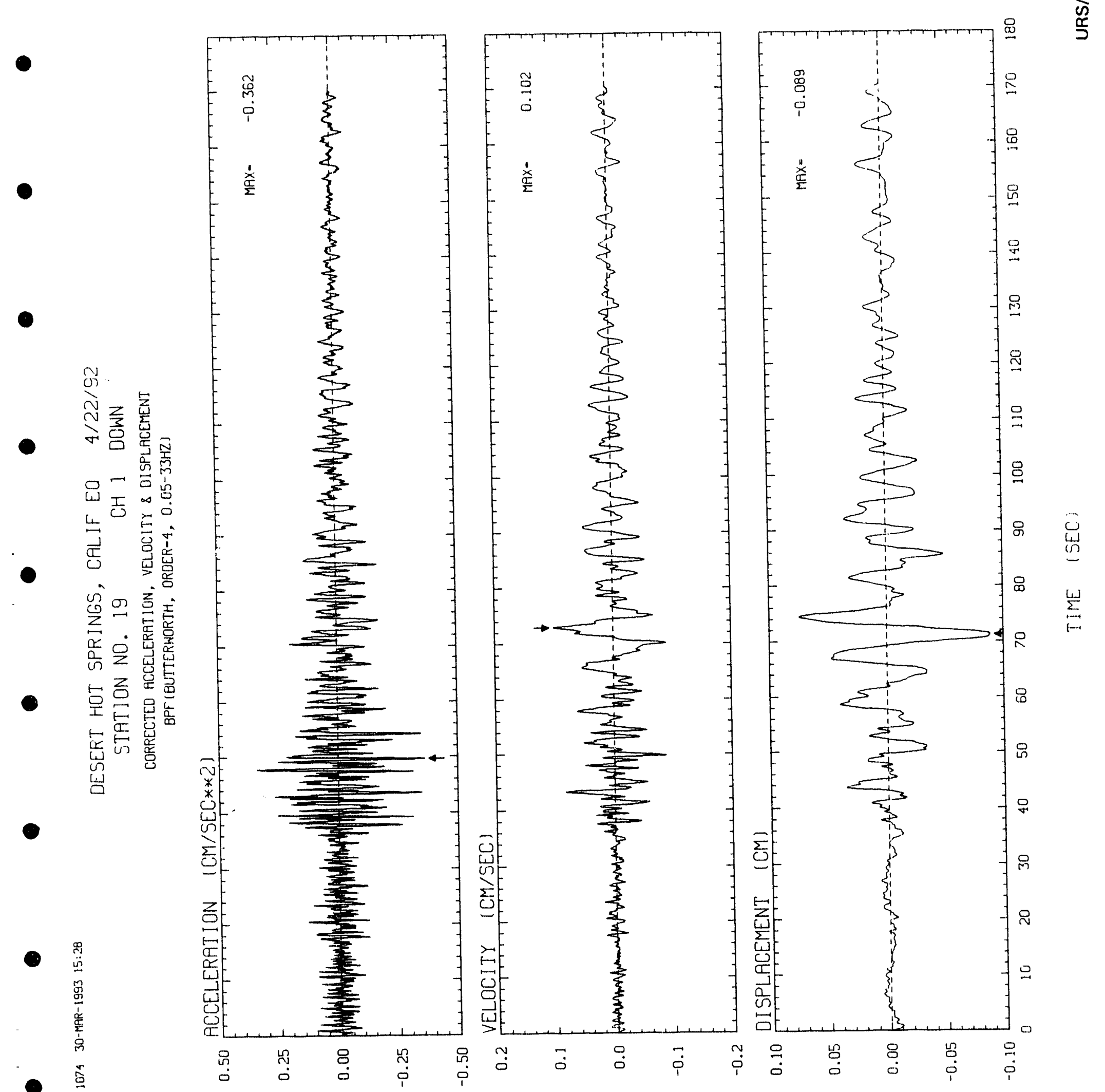


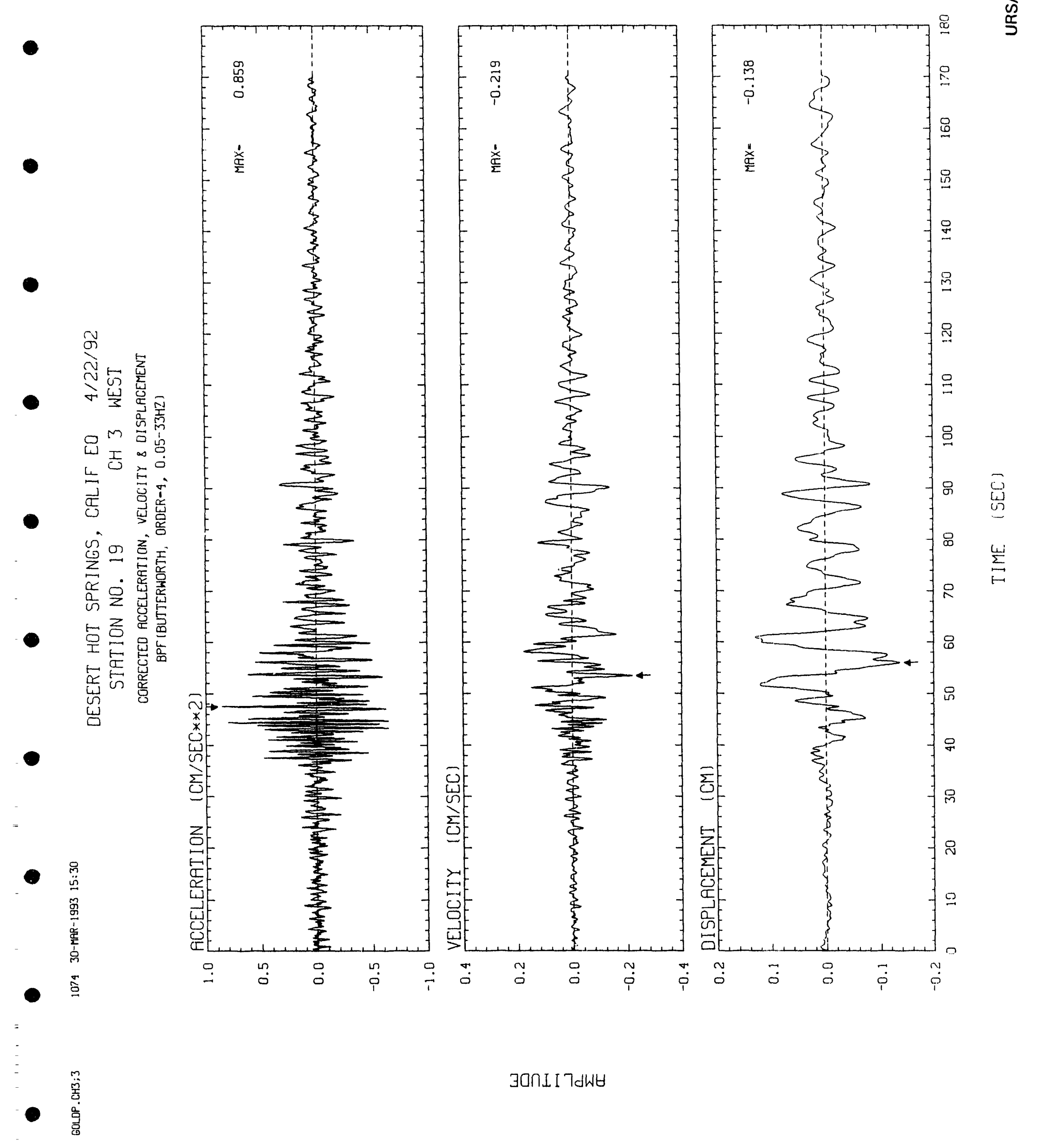



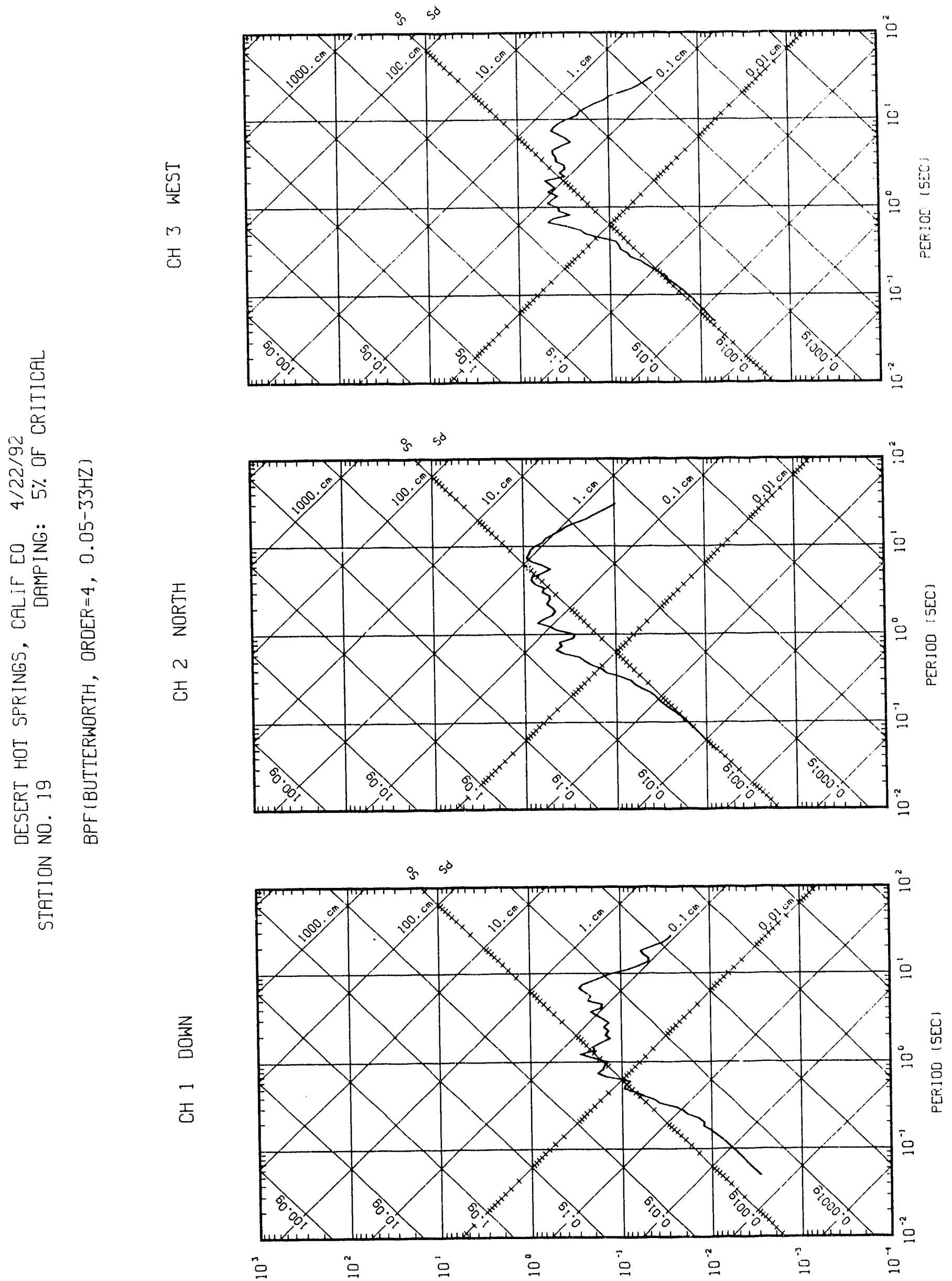

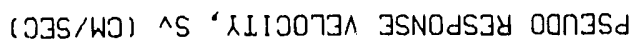


DISTRIBUTION LIST: JAB-10733-TM7

U.S. Department of Energy

Nevada Operations Office

P.O. Box 98518

Las Vegas, NV 89193-8518

J.E. O'Donnell

Technical Information Resource Center

U.S. Department of Energy

Office of Science Education and

Technical Information

P.O. Box 62

Oak Ridge, TN 37831

(2 Copies) 

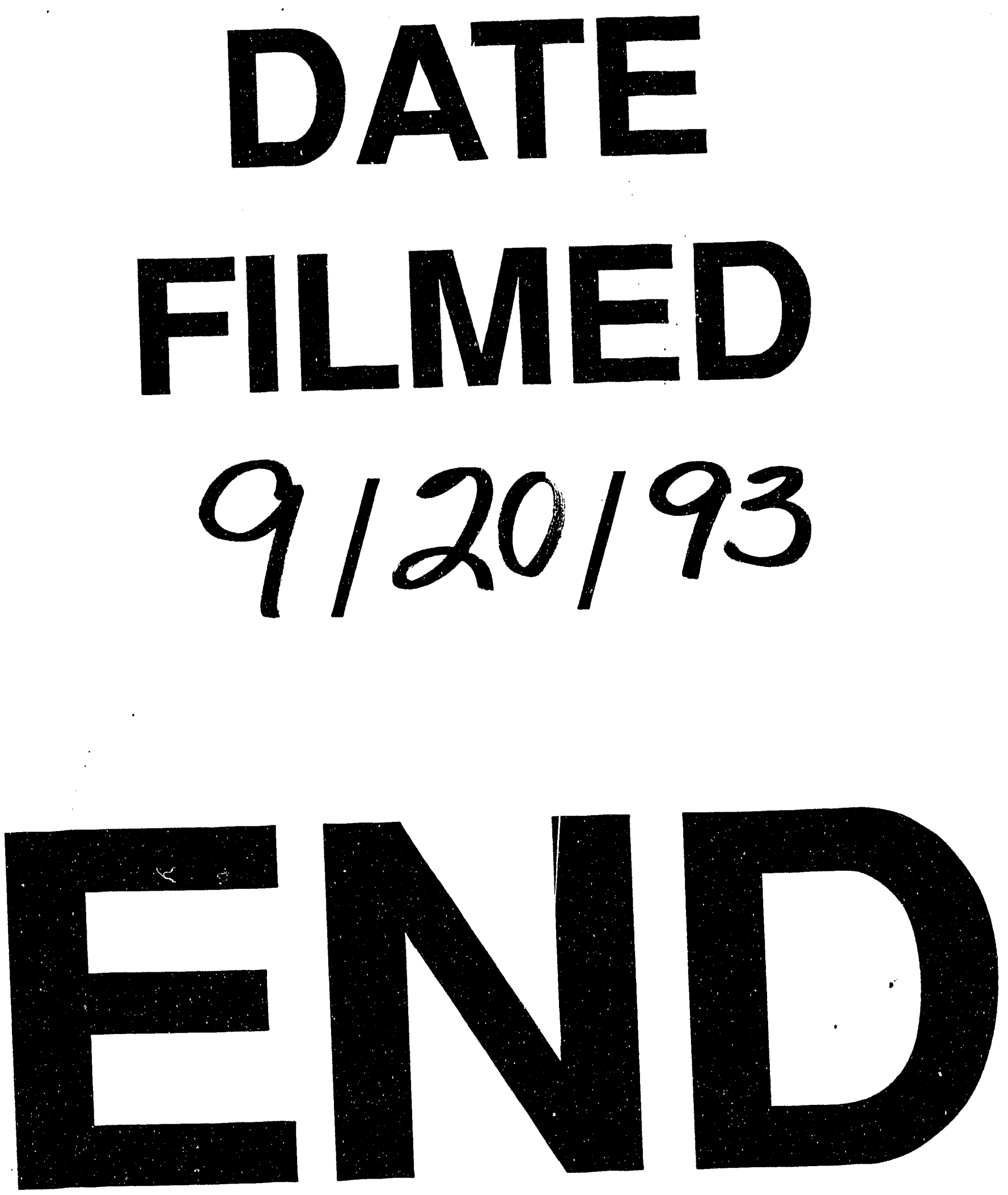
